LA-14389

Approved for public release;

distribution is unlimited.

Property and Lifetime Prediction in Aged U-Nb Alloys:

A Statistical Assessment

- Los Alamos 
Edited by Mable Amador, Group IRM-CAS.

Photocomposition by Deidre' A. Plumlee, Group IRM-CAS.

Los Alamos National Laboratory, an affirmative action/ equal opportunity employer, is operated by Los Alamos National Security, LLC, for the National Nuclear Security Administration of the U.S. Department of Energy under contract DE-AC52-06NA25396.

This report was prepared as an account of work sponsored by an agency of the U.S. Government. Neither Los Alamos National Security, LLC, the U.S. Government nor any agency thereof, nor any of their employees make any warranty, express or implied, or assume any legal liability or responsibility for the accuracy, completeness, or usefulness of any information, apparatus, product, or process disclosed, or represent that its use would not infringe privately owned rights. Reference herein to any specific commercial product, process, or service by trade name, trademark, manufacturer, or otherwise does not necessarily constitute or imply its endorsement, recommendation, or favoring by Los Alamos National Security, LLC, the U.S. Government, or any agency thereof. The views and opinions of authors expressed herein do not necessarily state or reflect those of Los Alamos National Security, LLC, the U.S. Government, or any agency thereof. Los Alamos National Laboratory strongly supports academic freedom and a researcher's right to publish; as an institution, however, the Laboratory does not endorse the viewpoint of a publication or guarantee its technical correctness. 
LA-14389

Issued: February 2009

\section{Property and Lifetime Prediction in}

Aged U-Nb Alloys: A Statistical Assessment

Robert E. Hackenberg

Geralyn M. Hemphill 
This page left blank intentionally. 


\section{TABLE OF CONTENTS}

LIST OF ACRONYMS AND SYMBOLS ........................................................................... ix

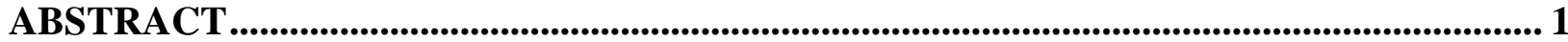

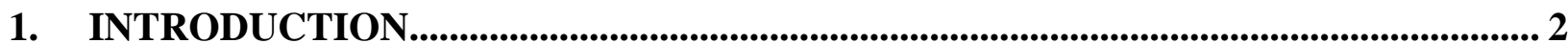

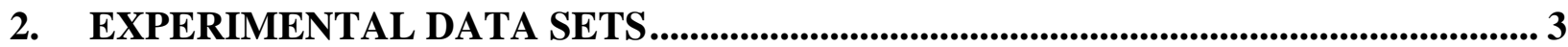

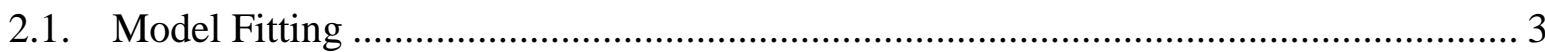

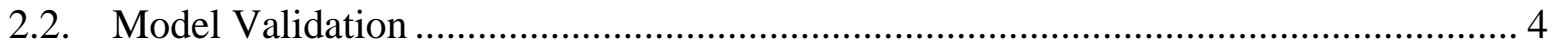

2.2.1. Nonbanded U-5.6Nb and U-7.7Nb .......................................................... 5

2.2.2. Banded U-6Nb ......................................................................................... 5

3. STATISTICAL EVALUATION METHOD ............................................................... 6

3.1. Sources and Magnitudes of Scatter................................................................................ 6

3.2. Previous Analysis Method ........................................................................................ 7

3.2.1. Modeling Approach .................................................................................. 7

3.2.2. Failure Criterion and Lifetime Prediction........................................................ 9

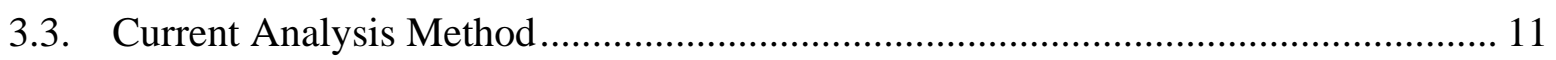

3.3.1. Statistical Modeling Overview ……………………..................................... 11

3.3.2. Model Evaluation......................................................................................... 12

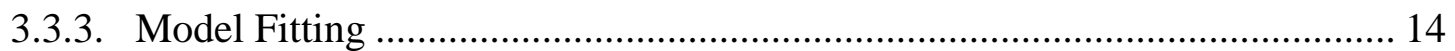

3.3.4. Model Predictions and Statistical Intervals................................................ 15

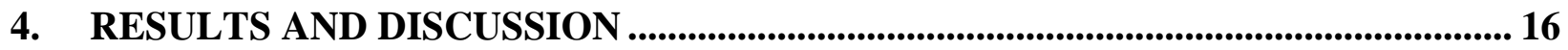

4.1. Model Fitting—General Comments ………………..................................................... 16

4.1.1. Quality of Fitting................................................................................... 16

4.1.2. Comparison of Parameters and Aging Responses ........................................... 18

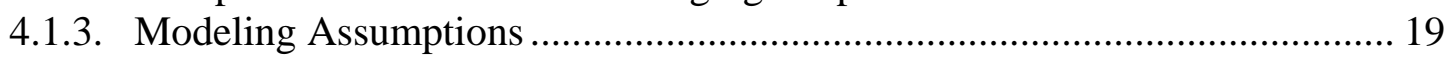

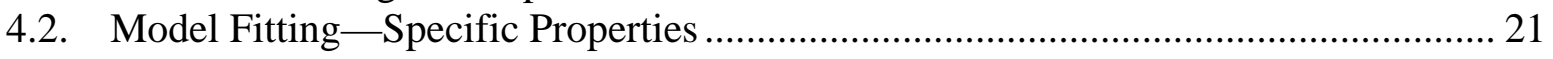

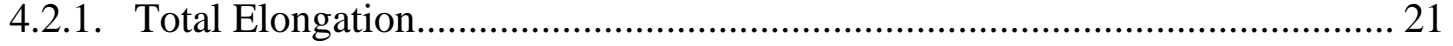

4.2.2. Uniform Elongation ................................................................................ 21

4.2.3. First-Yield Strength ................................................................................ 21

4.2.4. First-Yield Modulus................................................................................ 22

4.2.5. Second-Yield Strength ............................................................................... 22

4.2.6. Ultimate Tensile Strength ............................................................................. 22

4.2.7. Vickers Hardness ........................................................................................ 22

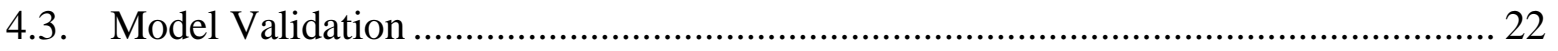

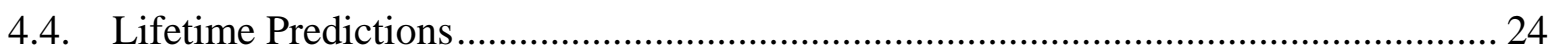

4.5. Evaluation of Age-Sensitive Properties for Surveillance ………………….............. 25

4.6. Recommendations for Future Work......................................................................... 26

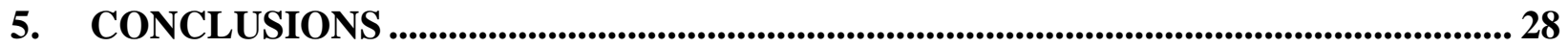

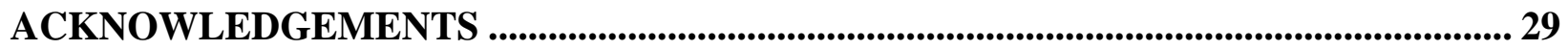

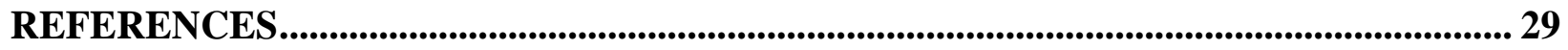

APPENDIX 1: SOURCES AND MAGNITUDES OF SCATTER ..................................... 101

APPENDIX 2: MODEL FITS AND PREDICTIONS ON AN ALTERNATE DEFINITION OF HARDNESS REPLICATES ........................................ 105

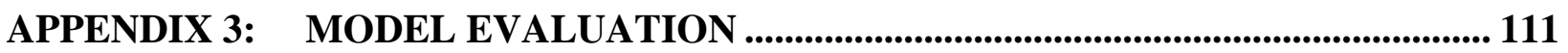

APPENDIX 4: BACKGROUND ON NONLINEAR MODELS .......................................... 121 


\section{LIST OF TABLES}

Table 2.1. Tensile Data from Each Replicate of Nonbanded U-5.6Nb. ............................31

Table 2.2. $\quad$ Vickers Microhardness Linescan Data from Nonbanded U-5.6Nb....................33

Table 2.3. Tensile Data from Each Replicate of Nonbanded U-7.7Nb.. ...........................35

Table 2.4. Vickers Microhardness Linescan Data from Nonbanded U-7.7Nb....................37

Table 2.5. Tensile Validation Data for Nonbanded U-7.7Nb. .........................................39

Table 2.6. $\quad$ Vickers Hardness Validation Data for Nonbanded U-5.6Nb............................40

Table 2.7. Vickers Hardness Validation Data for Nonbanded U-7.7Nb..............................41

Table 2.8. Chemical Analysis Results of Banded U-6Nb from the Validation

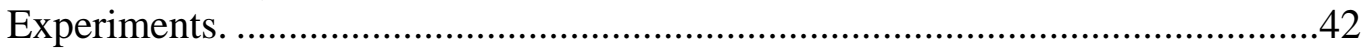

Table 2.9. Tensile Validation Data for Banded U-6Nb. ...............................................43

Table 2.10. Vickers Hardness Validation Data for Banded U-6Nb.....................................44

Table 3.1. $\quad$ Property Values at the Start and End (Peak) of Age-Hardening. .......................48

Table 3.2. Kinetic Parameters for U-5.6Nb Obtained from the Previous Study...................49

Table 3.3. Kinetic Parameters for U-7.7Nb Obtained from the Previous Study..................49

Table 3.4. Lifetimes for U-5.6Nb and U-7.7Nb Obtained from the Previous Study ...........49

Table 3.5. Analytic Method Comparison between the Previous and Present Studies..........50

Table 3.6. Total Elongation Kinetic Parameters for U-5.6Nb. ...........................................51

Table 3.7. Total Elongation Kinetic Parameters for U-7.7Nb.........................................51

Table 3.8. Uniform Elongation Kinetic Parameters for U-5.6Nb.....................................51

Table 3.9. Uniform Elongation Kinetic Parameters for U-7.7Nb.......................................51

Table 3.10. First-Yield Strength Kinetic Parameters for U-5.6Nb....................................52

Table 3.11. First-Yield Strength Kinetic Parameters for U-7.7Nb. ....................................52

Table 3.12. $\quad$ First-Yield Modulus Kinetic Parameters for U-5.6Nb. ...................................52

Table 3.13. First-Yield Modulus Kinetic Parameters for U-7.7Nb ....................................52

Table 3.14. Second-Yield Strength Kinetic Parameters for U-5.6Nb................................53

Table 3.15. Second-Yield Strength Kinetic Parameters for U-7.7Nb..................................53

Table 3.16. Ultimate Tensile Strength Kinetic Parameters for U-5.6Nb.............................53

Table 3.17. Ultimate Tensile Strength Kinetic Parameters for U-7.7Nb.............................53

Table 3.18. Vickers Hardness Kinetic Parameters for U-5.6Nb.........................................54

Table 3.19. Vickers Hardness Kinetic Parameters for U-7.7Nb..........................................54

Table 4.1. Results for Three Quality-of-Model-Fitting Metrics Evaluated for each Property

in $\mathrm{U}-5.6 \mathrm{Nb}$..............................................................................................55

Table 4.2. $\quad$ Results for Three Quality-of-Model-Fitting Metrics Evaluated for each Property

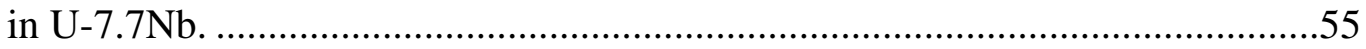

Table 4.3. Residual Standard Errors and Ancillary Quantities .........................................55

Table 4.4. Summary of Activation Energies Q from Model Fitting of the Previous Study and

This Study................................................................................................56

Table 4.5. Summary of A Parameter (= $1 / \mathrm{dx})$ from Model Fitting of the Previous Study and

This Study.....................................................................................................56

Table 4.6. Summary of B Parameter $\left(=x_{0}\right.$ at $\left.300^{\circ} \mathrm{C}\right)$ from Model Fitting of the Previous

Study and This Study...............................................................................56

Table 4.7. Experimentally Measured and Predicted Mean TE Values for Various U-Nb

Alloys for Current and Future Model Validation. .............................................57

Table 4.8. Experimentally Measured and Predicted Mean UE Values for Various U-Nb

Alloys for Current and Future Model Validation. ...............................................58 
Table 4.9. Experimentally Measured and Predicted Mean 1YS Values for Various U-Nb Alloys for Current and Future Model Validation. ............................................59

Table 4.10. $\quad$ Experimentally Measured and Predicted Mean 1YM Values for Various U-Nb Alloys for Current and Future Model Validation. .............................................60

Table 4.11. Experimentally Measured and Predicted Mean 2YS Values for Various U-Nb Alloys for Current and Future Model Validation. .............................................61

Table 4.12. Experimentally Measured and Predicted Mean UTS Values for Various U-Nb Alloys for Current and Future Model Validation. ..............................................62

Table 4.13. Experimentally Measured and Predicted Mean HV Values for Various U-Nb Alloys for Current and Future Model Validation. ..............................................63

Table 4.14. Lifetime Predictions for U-5.6Nb..........................................................64

Table 4.15. Lifetime Predictions for U-7.7Nb.............................................................64

Table 4.16. Threshold of Fractional and Absolute Property Changes, and Associated Aging Times at $40^{\circ} \mathrm{C}$ where a Statistically Significant Aging Response is Expected to be Experimentally Observable in Banded U-6Nb. .65

Table 4.17. Threshold of Fractional and Absolute Property Changes, and Associated Aging Times at $40^{\circ} \mathrm{C}$ where a Statistically Significant Aging Response is Expected to be Experimentally Observable in Nonbanded U-5.6Nb. .65

Table 4.18. Threshold of Fractional and Absolute Property Changes, and Associated Aging Times at $40^{\circ} \mathrm{C}$ where a Statistically Significant Aging Response is Expected to be Experimentally Observable in Nonbanded U-7.7Nb. .65

Table 4.19. Notional Tensile Property Equivalency Table that Would Result from a Future Systematic Study of the Effects of Tensile Geometry and Machining Damage. .66

\section{LIST OF FIGURES}

Figure 2.1. Cylindrical tensile specimen geometry used for the nonbanded U-5.6Nb and U-7.7Nb studies for both model fitting and for validation.

Figure 2.2. Cylindrical tensile specimen geometry used for the banded U-6Nb validation

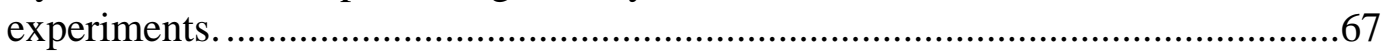

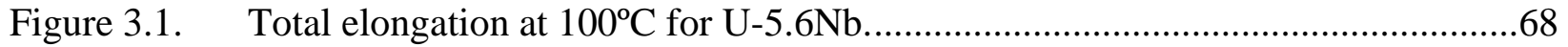

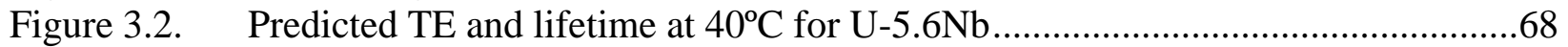

Figure 4.1. Universal plot of U-5.6Nb TE data (points) and model fit to data (solid line).....69

Figure 4.2. Universal plot of U-7.7Nb TE data (points) and model fit to data (solid line).....69

Figure 4.3. Universal plot of U-5.6Nb UE data (points) and model fit to data (solid line)....70

Figure 4.4. Universal plot of U-7.7Nb UE data (points) and model fit to data (solid line)....70 Figure 4.5. Universal plot of U-5.6Nb 1YS data (points) and model fit to data (solid line)....71 Figure 4.6. Universal plot of U-7.7Nb 1YS data (points) and model fit to data (solid line)....71 Figure 4.7. Universal plot of U-5.6Nb 1YM data (points) and model fit to data (solid line). .72 Figure 4.8. Universal plot of U-7.7Nb 1YM data (points) and model fit to data (solid line). .72 Figure 4.9. Universal plot of U-5.6Nb 2YS data (points) and model fit to data (solid line)....73 Figure 4.10. Universal plot of U-7.7Nb 2YS data (points) and model fit to data (solid line)...73 Figure 4.11. Universal plot of U-5.6Nb UTS data (points) and model fit to data (solid line)...74 Figure 4.12. Universal plot of U-7.7Nb UTS data (points) and model fit to data (solid line)...74 Figure 4.13. Universal plot of U-5.6Nb HV data (points) and model fit to data (solid line).....75 
Figure 4.14. Universal plot of U-7.7Nb HV data (points) and model fit to data (solid line).....75

Figure 4.15. Consolidated aging response model fits at $200^{\circ} \mathrm{C}$ for (a) $\mathrm{U}-5.6 \mathrm{Nb}$ and

(b) U-7.7Nb. .76

Figure 4.16. Consolidated aging response predictions at $40^{\circ} \mathrm{C}$ for (a) $\mathrm{U}-5.6 \mathrm{Nb}$ and

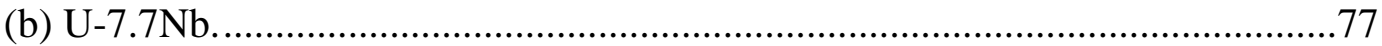

Figure 4.17. U-5.6Nb TE data, model fits to data, and low-temperature model predictions....78

Figure 4.18. U-7.7Nb TE data, model fits to data, and low-temperature model predictions.....79

Figure 4.19. U-5.6Nb UE data, model fits to data, and low-temperature model predictions.....80

Figure 4.20. U-7.7Nb UE data, model fits to data, and low-temperature model predictions.....81

Figure 4.21. U-5.6Nb 1YS data, model fits to data, and low-temperature model predictions...82

Figure 4.22. U-7.7Nb 1YS data, model fits to data, and low-temperature model predictions...83

Figure 4.23. U-5.6Nb 1YM data, model fits to data, and low-temperature model predictions. 84

Figure 4.24. U-7.7Nb 1YM data, model fits to data, and low-temperature model predictions. 85

Figure 4.25. U-5.6Nb 2YS data, model fits to data, and low-temperature model predictions...86

Figure 4.26. U-7.7Nb 2YS data, model fits to data, and low-temperature model predictions...87

Figure 4.27. U-5.6Nb UTS data, model fits to data, and low-temperature model predictions ..88

Figure 4.28. U-7.7Nb UTS data, model fits to data, and low-temperature model predictions. .89

Figure 4.29. U-5.6Nb HV data, model fits to data, and low-temperature model predictions....90

Figure 4.30. U-7.7Nb HV data, model fits to data, and low-temperature model predictions ....91

Figure 4.31. U-5.6Nb validation HV data and model predictions ......................................92

Figure 4.32. $\quad$ U-7.7Nb validation TE data and model predictions ......................................93

Figure 4.33. U-7.7Nb validation UE data and model predictions .....................................93

Figure 4.34. U-7.7Nb validation 1YS data and model predictions .....................................94

Figure 4.35. U-7.7Nb validation 1YM data and model predictions.....................................94

Figure 4.36. U-7.7Nb validation 2YS data and model predictions ....................................95

Figure 4.37. U-7.7Nb validation UTS data and model predictions.....................................95

Figure 4.38. U-7.7Nb validation HV data and model predictions ........................................96

Figure 4.39. U-6Nb validation TE data and model predictions ........................................99

Figure 4.40. U-6Nb validation UE data and model predictions .........................................97

Figure 4.41. U-6Nb validation 1YS data and model predictions ......................................98

Figure 4.42. $\quad$ U-6Nb validation 1YM data and model predictions ......................................98

Figure 4.43. U-6Nb validation 2YS data and model predictions .........................................99

Figure 4.44. U-6Nb validation UTS data and model predictions........................................99

Figure 4.45. U-6Nb validation HV data and model predictions. .........................................100 


\section{LIST OF ACRONYMS AND SYMBOLS}

$\alpha$

$\alpha^{\prime \prime}$

$\gamma$

$\gamma_{2}$

$\gamma^{0}$

$\%$ RA

03K-422

03K-425

1YS

1YM

2YS

A

$\mathrm{AM}$

AQ

$\mathrm{A}_{\mathrm{s}}$

at.\%

B

BCC

CI

$d f$

$d x$

$f$

$f^{\prime}$

HV

LANL

MA

$n$

$n v$

$p$

$P$

$P($ peak $)$
Equilibrium orthorhombic phase found in pure and alloyed uranium

Monoclinic phase found in alloyed uranium

Body-centered cubic phase found in pure and alloyed uranium

Equilibrium body-centered cubic phase containing approximately 75 at.\% $\mathrm{Nb}$

Tetragonal phase found in uranium alloys, a distorted form of $\gamma$

Percent reduction in area

LANL/MST-6 foundry identification number for the U-7.7Nb plate

LANL/MST-6 foundry identification number for the U-5.6Nb plate

First-yield strength

First-yield modulus

Second-yield strength

Adjustable parameter for various nonlinear models

Annealed-then-machined condition; also referred to as "as-machined”

As quenched, same as stating "time $=0$, MA condition"

Austenite start temperature

Atomic percent

Adjustable parameter for various nonlinear models

Body-centered cubic

Confidence interval

Degrees of freedom

Adjustable parameter for the Boltzmann model

Fractional property change referred to $P$ (start)

Fractional property change referred to $P(t=0)$

Vickers Microhardness

Los Alamos National Laboratory (Los Alamos, NM)

Machined-then-annealed condition; also referred to as “as-annealed”

Number of replicate data points

Number of validation data points

Number of adjustable parameters

Generic age-sensitive property; also a $3 \times 1$ vector containing the first partial derivatives for the estimates of $Q, A$, and $B$ (Appendix 4 only)

$\mathrm{P}$ at the end of the age-hardening regime, at peak age 


\begin{tabular}{ll}
$P($ start $)$ & P at the start of the age-hardening regime \\
$P(t=0)$ & P at aging time $=0$, evaluated condition (AM vs MA) unspecified \\
$P(t=0, A M)$ & P at aging time $=0$ evaluated in the as-machined condition \\
$P(t=0, M A)$ & P at aging time $=0$ evaluated in the as-annealed condition, same as $P(A Q)$ \\
$P I$ & Prediction interval \\
pred $_{i}$ & Predicted mean value for the time $i$ \\
$Q$ & Apparent activation energy for an aging or other physical process \\
Qdiff & Activation energy for diffusion \\
QMU & Quantification of margins and uncertainties \\
$R$ & Universal gas constant \\
RFP & Rocky Flats Plant \\
$R S E$ & Residual standard error \\
$R S S$ & Residual sum-of-squares \\
$S D$ & Standard Deviation \\
$s e . f i t_{i}$ & Standard error of a fitted value at time $i$ \\
$t$ & Time; also a generic t-statistic \\
$t(n, d f)$ & t-statistic for a 100 $\times n$ \% confidence interval with $d f$ degrees of freedom \\
$T$ & Temperature \\
TE & total (plastic) elongation (engineering strain) to tensile failure \\
UE & uniform (plastic) elongation (engineering strain) to tensile failure \\
UTS & Ultimate tensile strength \\
VAR & Vacuum arc remelt(ed) \\
wt\% & Weight percent \\
$x$ & log ${ }_{10}$ (time in minutes) \\
$x^{\prime}$ & log ${ }_{10}$ (equivalent time in minutes at a reference temperature $T_{2}$ ) \\
$x_{o}$ & Adjustable parameter for the Boltzmann model \\
$y$ & Generic response variable \\
Y-12 & Oak Ridge Y-12 Plant (Oak Ridge, TN) \\
& \\
\hline &
\end{tabular}




\title{
Property and Lifetime Prediction in Aged U-Nb Alloys: A Statistical Assessment
}

by

\author{
Robert E. Hackenberg and Geralyn M. Hemphill
}

\begin{abstract}
This study was undertaken to better model the aging response of U-Nb alloys, particularly to predict properties and their scatter bands, from which lifetimes and their uncertainties can be evaluated. Predictive models of the aging time- and temperaturedependencies of seven age-sensitive properties were developed for nonbanded U-5.6 wt\% Nb and U-7.7 wt\% Nb alloys. These properties were total and uniform plastic tensile elongation to failure; first-yield, second-yield, and ultimate tensile strengths; first-yield elastic modulus; and Vickers microhardness. A more systematic and statistically aware kinetics modeling approach than employed previously gave reasonable models fits to accelerated aging property data in nonbanded U-5.6Nb and U-7.7Nb, and useful predictions for most of the properties studied. With minor modifications, the U-5.6Nb model was extended to banded U-6Nb. This modeling approach shared many of the key assumptions of the previous approach, including the assumption of Arrhenius behavior and the use of three adjustable parameters. Initial data returns from long-term aging experiments were used to validate the fitted models, a new feature to this study. The apparent activation energies of aging for the property of greatest interest, total elongation, were $32 \mathrm{kcal} / \mathrm{mol}$ for $\mathrm{U}-5.6 \mathrm{Nb}$ and $39 \mathrm{kcal} / \mathrm{mol}$ for U-7.7Nb, respectively; those for the other properties spanned $14-51 \mathrm{kcal} / \mathrm{mol}$. Comparing the goodness of the model fits for the seven properties, the best fits were obtained for second-yield strength and hardness, the first-yield modulus fit the least well, and the other properties' fits were in between. The U-5.6Nb models are more robust and therefore are expected to have better predictive power than those of U-7.7Nb, especially at the lower aging temperatures of interest. Model extrapolations to longer times (up to 5 years) and lower temperatures (as low as $40^{\circ} \mathrm{C}$ ) than those used for the model fitting agreed well with most of the validation data gathered for both nonbanded alloys, as well as banded U-6Nb, giving provisional validation of the fitted models. Property predictions for planned or already pending validation experiments are also provided. With a view towards enabling future modeling efforts, this report tabulates all replicate tensile properties and complete hardness scan data used for both model fitting and validation. For surveillance purposes, the properties most practically amenable to detecting the onset of aging at the earliest times are first-yield strength and second-yield strength. Even at aging temperatures as high as $60^{\circ} \mathrm{C}$, the minimum lifetimes from this present study are beyond 100 years, giving no cause for concern, if the previously developed failure criterion based on uniaxial tensile elongation (with its caveats) is accepted.

\section{This document must be reproduced in COLOR to ensure accuracy of the figures.}




\section{INTRODUCTION}

There is concern that aging during long-term stockpile storage of U-6 wt\% $\mathrm{Nb}$ alloy components will change the material in ways that will adversely affect their performance. In this context, "aging” is a generic term that can encompass a variety of specific physical mechanisms by which the microstructure and properties evolve as a function of time for a given temperature and local environment. U-6Nb ${ }^{1}$ is a complex material because of its gross compositional inhomogeneity (its chemical banding spans 4-8 wt\%), its metastable starting microstructure, and the fact that a variety of external factors such as temperature, stress, and gaseous species can cause aging through multiple mechanisms. In principle, such mechanisms can operate in overlapping timetemperature domains, at different rates, and with different signs and magnitudes of resulting property changes. These aging mechanisms have an aggregate effect on the properties (usually adversely) and ultimately the performance, but their deconvolution is not simple.

A previous report [2007hac2] assessed several different aging mechanisms and concluded that the most significant of these was the phase separation into $\mathrm{Nb}$-rich and $\mathrm{Nb}$-lean regions as the initially metastable, Nb-supersaturated $\alpha$ " microstructure evolves toward the equilibrium twophase mixture of $\alpha+\gamma_{2}$. These processes give rise to age hardening, phenomenologically defined as increasing hardness and strength and decreasing ductility observed as a function of increasing aging time-at-temperature. There continues to be uncertainty as to the specific physical mechanism of age hardening at temperatures relevant to U-6Nb material processing $\left(\leq 200^{\circ} \mathrm{C}\right)$ and stockpile storage $\left(\leq 60^{\circ} \mathrm{C}\right)$ [2008cla]. There is also uncertainty regarding the nature and magnitude of the failure criterion. These limitations make the most desirable approach of property response and lifetime prediction — that based on fundamental physics — unattainable at the present time.

Therefore, the next best approach, a semi-empirical one, was taken to model the phenomenological property evolution during aging, which enabled lifetime estimates to be made from an assumed failure criterion couched in terms of one or more of these age-sensitive properties. It should be noted that the predictions of age-sensitive properties are useful not just for lifetime prediction, but also as a tool that can be applied to U-6Nb component surveillance studies and also to "what-if" analyses. Drawing upon a large body of artificial aging data obtained from nonbanded U-5.6Nb and U-7.7Nb material [2007hac1], an earlier report [2007hac2] determined the aging (property evolution) kinetics as a function of time and temperature in terms of an Arrhenius model [1976eck, 2002eck], and used this to make a first prediction of banded U-6Nb component lifetimes.

The main purpose of this study was to provide a more statistically aware quantification of these property and lifetime predictions and uncertainties using the same body of model-fitting data. A secondary end was to expand the number of age-sensitive properties (from one to seven) that could be predicted with an aging model, which has relevance for the types of surveillance on U-6Nb components that might be contemplated. Finally, this report serves the tertiary purpose of archiving, in tabular format, all replicate tensile properties and complete hardness scan data used for both model fitting and validation. Aware that new aging data and scientific insights will likely become available in the future (e.g., from planned or already pending long-term validation experiments), this archiving is done with a view toward supporting future reanalyses of an expanded body of data.

\footnotetext{
${ }^{1}$ Alloy and phase compositions in this report are given in weight percent (wt\%) unless otherwise specified.
} 
The basic assumptions underlying this present analysis are the same as those of the previous study, particularly with regards to the fundamental axes by which aging is measured and analyzed:

1. Time-axis: log(aging time) is the relevant time unit of aging.

2. Temperature-axis: the Arrhenius formalism was used to compare the kinetics at different aging temperatures, for both model fitting and interpolation/extrapolation.

3. Property-axis: age-related property changes were normalized to a fractional property change spanning $0 \leq f \leq 1$, using a linear transformation of the property $P$ to the fractional change $f$.

Early in this study, these three assumptions were compared against credible alternative time-, temperature- and property-scalings, with the result that the original assumptions yielded the most satisfactory results. Aging time, aging temperature, and alloy composition (Nb content) were the independent variables in this study, and the various property responses were the dependent variables.

It should be noted at the outset that, although considerable uncertainty exists with respect to the quantitative magnitude of the failure criterion (and even its conceptual validity), no attempt was made to quantify the uncertainty in the failure criterion; the failure criterion was accepted simply "as is." In this light, it should be kept in mind that this study is meant only to quantify property predictions, lifetime predictions, and their statistical uncertainties. Given the doubt surrounding the magnitude and conceptual validity of the failure criterion, a further analysis involving property/performance margin calculations (as would be done in a bona fide Quantification of Margins and Uncertainties (QMU) study) could not be justified, and therefore was not undertaken in this study, although the information contained in this report is sufficient for such calculations, provided they are appropriately hedged by these caveats.

\section{EXPERIMENTAL DATA SETS}

\subsection{Model Fitting}

The data set used for aging kinetics model fitting was from the nonbanded U-5.6Nb (plate ID 03K-425) and U-7.7Nb (plate ID 03K-422) material, whose synthesis was described in [2007hac1]. These two nonbanded materials, which are compositionally homogeneous with respect to $\mathrm{Nb}$, were used to represent the average (U 5.8Nb) and upper limit (U 8Nb) of compositions present on 100-200 micron length scales in the banded U-6Nb material. The high$\mathrm{Nb}$ alloy was selected for study because it is considered to age faster than the mean (U 5.8Nb) or lower limit (U 4Nb) compositions of the banded U-6Nb [2002eck]. Therefore, there was concern that the high-Nb bands would degrade faster and cross the failure threshold sooner than the mean or lower-Nb bands, which on a conservative performance assessment would mean that the high-Nb band behavior would be the life-limiting factor for banded U-6Nb.

Tensile and microhardness specimens were machined from the U-5.6Nb and U-7.7Nb plates, solution heat treated 30 minutes in the $\gamma$-bcc single-phase field $\left(800^{\circ} \mathrm{C}\right.$ and $850^{\circ} \mathrm{C}$, respectively) and water quenched in order to erase any memory of previous aging or machining damage. This is referred to as the "time $=0$, machined-then-annealed $(\mathrm{t}=0, \mathrm{MA})$," "as-annealed," or "asquenched (AQ)" condition, a material state that allows the aging behavior to be observed without complications from machining damage [2007hac2]. Figure 2.1 provides the tensile specimen 
geometry. The strain was measured with an extensometer of $0.5^{\prime \prime}$ length. About six tensiles were tested in the AQ condition within six hours of the quenching operation to minimize any effects of ambient-temperature aging (effects of which are expected to be insignificant in any event). The remainder of the specimens were subsequently aged at $100^{\circ} \mathrm{C}, 200^{\circ} \mathrm{C}, 250^{\circ} \mathrm{C}$, and $300^{\circ} \mathrm{C}$ for various times, up to 140 days. In this context, age is defined as time-at-temperature, e.g., $100^{\circ} \mathrm{C}$ for 1,000 minutes.

The same data set as that used for the previous report [2007hac2] was analyzed in this present study. Unlike the previous report, Tables 2.1-2.4 provide all of the replicate data, not just averages and standard deviations for each aging condition. For any given age, the number of tensile specimens (= number of replicate measurements $n$ ) varied from 2-4. For any given age, a single metallographic coupon was used for Vickers microhardness (HV) evaluation; 7-15 measurements were taken from each coupon. In the HV data (Tables 2.2 and 2.4), note that the linescan used was actually a zig-zag trace to ensure proper separation between neighboring indents, especially in the majority of instances where a 500-gram load was used.

Data from all ages were used in the model fitting of both the previous and current studies except for those from ages suspected to be overaged. A softening in one or more properties (i.e., increasing elongation and/or decreasing hardness, strength, or modulus) beyond the agehardening regime constituted indirect evidence of overaging. Microstructural evaluation of these aged specimens failed to reveal direct signs of classical overaging in U -Nb alloys — cellular decomposition products consuming the matrix phase [1976eck, 1984eck] — therefore, the apparent softening could be merely the result of scatter in the data or a "pause" in the hardening reaction, for example, arising from the dissolution of solute clusters or metastable precipitates. To err on the conservative side, indirect evidence of overaging was deemed sufficient to warrant exclusion from model fitting. Such ages include the following:

1. U-5.6Nb: 100,000 minutes at both $250^{\circ} \mathrm{C}$ and $300^{\circ} \mathrm{C}$, and

2. U-7.7Nb: 10,000 minutes and 100,000 minutes at $300^{\circ} \mathrm{C}$.

These overaged data are included in Tables 2.1 to 2.4 for the sake of completeness, with this caveat noted. It should be noted that no data points potentially associated with an initial softening transient at short times and low temperatures (before the onset of bona fide age hardening; see Section 3.2) were excluded from model fitting because the nature and extent of such a prehardening transient was not clearly enough defined, given the paucity of data and their large error bars.

\subsection{Model Validation}

Long-term aging experiments were initiated to test the predictive power of the improved aging model description developed in this study. These validation aging experiments probed longer times ( $\geq 221$ days) and (in some instances) lower temperatures $\left(\leq 100^{\circ} \mathrm{C}\right)$ than the aging data used to fit the aging kinetics model ( $\leq 140$ days and $\geq 100^{\circ} \mathrm{C}$, respectively), therefore providing a more rigorous test of the model's extrapolative power. The initial data return from these experiments is presented here; more complete reports are planned in the next year or two to document more of this data that will soon become available, and in more detail (e.g., with complete stress-strain curves and microstructural and other characterization.) These validation studies are divided into two parts. 


\subsubsection{Nonbanded $U-5.6 N b$ and $U-7.7 N b$}

The first set of validation data is from the same nonbanded U-5.6Nb and U-7.7Nb material that was employed previously for model fitting (Section 2.1.) Specimens were aged for 221 days (exactly 5,300 hours) at $100^{\circ} \mathrm{C}, 200^{\circ} \mathrm{C}$, and $300^{\circ} \mathrm{C}$. Even longer ages still in progress are anticipated to provide two-year and six-year aging data at these temperatures. These aging treatments were done in an identical manner to those documented in Section 2.1, namely, in the MA state and under encapsulation protection with an inert-gas (Ar) backfill. Because of specimen limitations, only a select number of the 221 day ages will have tensile data (Table 2.5), though hardness data were collected for all these aging conditions (Tables 2.6 and 2.7). Tensile data were collected in the same manner as in Section 2.1, with the specimen geometry of Figure 2.1 and an extensometer of 0.5 " length.

\subsubsection{Banded $U-6 N b$}

The second set of validation data is from banded U-6Nb of Rocky Flats Plant (RFP) pedigree. The material was twice vacuum arc remelted (VAR) and then hot worked (in the gamma-phase region) as follows: upset forged $50 \%$ and subsequently rolled $\sim 90 \%$ over a number of passes while changing the rolling direction between each pass, according to a standard schedule. Portions of this plate $4^{\prime \prime} \times 3^{\prime \prime} \times 0.5^{\prime \prime}$ in size were solutionized $800^{\circ} \mathrm{C}$ for two hours in a furnace with inert atmosphere and then oil quenched. ${ }^{2}$ Separate plates were then aged at $40^{\circ} \mathrm{C}, 65^{\circ} \mathrm{C}$, and $90^{\circ} \mathrm{C}$ in air furnaces with no atmosphere control. Chemical analysis results (Table 2.8) indicate no significant changes in the metal or light-element content in 5-year-aged material (vs unaged), indicating that aging in air at these low temperatures is not detrimental to the material from an environmental degradation point of view. In particular, note the statistically indistinguishable oxygen contents, and also the low hydrogen contents for all conditions, which are too low to alter the age-sensitive properties of interest to this study. Finally, we note that the bulk $\mathrm{Nb}$ content was measured to be $6.3 \mathrm{wt} \%$, which is at the upper end of the bulk (mean) Nb composition range expected in banded U-6Nb material, though not out of bounds. (No chemical analysis information was available from RFP.) Lacking significant aging data for alloys with $6.3 \mathrm{wt} \% \mathrm{Nb}$, the kinetic description (of fractional property change) generated from $5.6 \mathrm{wt} \% \mathrm{Nb}$ aging data will be used to predict the response of this banded U-6Nb material (6.3 wt\% measured composition). The only modification to the analysis as a result of the actual differences in $\mathrm{Nb}$ content will be a minor adjustment of the starting property values to those measured for the specific alloy (to be described later, and documented in Table 3.1).

After aging for a prescribed time, the plate was pulled from the furnace, and specimens for tensile testing (3 replicates), hardness testing, and chemical analysis were machined out of a 2"-long portion of the initially 4"-long plate (the other half was returned to the furnace for continued aging). Data from 5-year-aged specimens were available for comparison at the time of this study; other ages still in progress are anticipated to provide 0.625-, 1.25-, 2.5-, 10-, 20-, and 40-year aging data at these temperatures. Note that the tensile specimen size (Figure 2.2) is larger in all dimensions than that used for the nonbanded U-5.6Nb and U-7.7Nb specimens (Figure 2.1), though both are round. Particularly, the gage length and diameter are 1.25" and $0.25^{\prime \prime}$ for this larger specimen vs $0.70^{\prime \prime}$ and $0.10^{\prime \prime}$, respectively, for the smaller specimen. The larger size specimen has the advantage of having less variability in measured tensile properties

\footnotetext{
${ }^{2}$ An oil quench is sufficiently rapid [1984eck] to obtain the same metastable $\alpha$ " starting microstructure and properties as those obtained from a water quench used for the other U-Nb compositions.
} 
between otherwise identically treated replicates compared to the smaller size. The factor-of-six larger gage cross-section ( $0.0491 \mathrm{in}^{2}$ vs $0.0079 \mathrm{in}^{2}$ ) also minimizes the magnitude of the machining damage effect [2007hac2]. This is an important point because these U-6Nb tensiles will be examined in the annealed-then-machined (AM) condition, different from the machinedthen-annealed (MA) condition of all other tensile data. On the view that machining damage strengthens material residing a small, fixed distance from the machined surface, a larger crosssection lowers the fraction of material hardened due to machining damage, which on a "rule-ofmixtures” approach amounts to a lowering of the total increment of machine-damage "hardening" versus a specimen with a smaller cross section. For practical reasons, the tensile tests were run approximately 90 days after the tensile specimens underwent final machining, such a time interval could have allowed some relief of machining damage. The tensile data are listed in Table 2.9; these include data from unaged control specimens that were otherwise identically handled. The strain was measured with an extensometer of 0.5 " length.

In spite of the differences in tensile geometry and machining damage condition between these banded U-6Nb tensile specimens and all the nonbanded U-5.6Nb and U-7.7Nb tensile specimens, the assumption was made that these two effects on property values because of these factors were small in relation to the effect on property values because of the intrinsic aging response. This is a debatable assumption, but was made in view of the paucity of data that would provide a direct conversion of any given tensile property value (e.g., first-yield strength) from one tensile geometry to another. A study of property equivalencies among different tensile geometries (and with machining damage condition as an added variable) is recommended for the future.

The hardness data from the $40^{\circ} \mathrm{C}, 65^{\circ} \mathrm{C}$, and $90^{\circ} \mathrm{C}$ aged material are listed in Table 2.10; these include data from unaged control specimens that were otherwise identically handled.

Significantly more hardness measurements were taken than in the nonbanded material to better average out the point-to-point changes in local hardness because of the $\mathrm{Nb}$ banding in this material; the scans were run through the entire $0.5^{\prime \prime}$ cross section of the metallographic coupon.

\section{STATISTICAL EVALUATION METHOD}

\subsection{Sources and Magnitudes of Scatter}

Only the scatter arising from the intrinsic material variability, model (curve) fitting, and model fidelity to the physical aging mechanism(s) were accounted for in the current analysis method (Section 3.3). Other experimental errors deemed to be less significant were not explicitly accounted for. For completeness, the origins and estimated magnitudes of all the known or expected sources of scatter are detailed in Appendix 1.

The scatter in the experimental data could be accounted for in the fitting methods in two different ways:

1. Fitting the average of the replicate points weighted by the standard deviation. This was used for the previous analysis method [2007hac2].

2. Fitting all the replicate data points, each replicate weighted equally. This was used in the current analysis method.

A replicate is defined as an individual measurement from a particular specimen of a given alloy and given aging condition (e.g., U-5.6Nb, $200^{\circ} \mathrm{C}, 1,000$ minutes, tensile specimen \#1). In the 
context of method 2 (above), the definition of what constitutes a replicate is important for weighting data during model fitting and determining prediction error bounds. In this work, it was assumed that a tensile replicate corresponded to the outcome from an individual tensile specimen; there were typically $2-4$ replicates per aging condition.

Defining the entity that constitutes a hardness replicate was less clear-cut, because multiple measurements (tens to perhaps even hundreds) can be taken from the same coupon. Two definitions were considered. One definition is that the average of numerous hardness measurements on an individual coupon constitutes a single replicate point. The alternate definition is that each indent from an individual hardness measurement is a replicate point, potentially giving tens or even hundreds of replicate points per coupon. The previous and present study assume the former; but Appendix 2 documents an alternative analysis assuming the latter.

\subsection{Previous Analysis Method}

\subsubsection{Modeling Approach}

The previous analysis method is documented in [2007hac2]. For the benefit of the reader, this methodology will be summarized. The aging response was tracked in U-5.6Nb and U-7.7Nb indirectly through the observed time-dependent changes in various mechanical properties generically referred to as $P($ time). These following age-sensitive properties were measured at ambient temperature following artificial ages at $100^{\circ} \mathrm{C}, 200^{\circ} \mathrm{C}, 250^{\circ} \mathrm{C}$, and $300^{\circ} \mathrm{C}$ :

1. Total (Plastic) Elongation (TE)

2. Uniform (Plastic) Elongation (UE)

3. First-Yield Strength (1YS)

4. First-Yield Modulus (1YM)

5. Second-Yield Strength (2YS)

6. Ultimate Tensile Strength (UTS)

7. Vickers Hardness (HV)

8. Percent Reduction in Area (\%RA)

The specific task common to both the previous and present study was to determine a best-fit analytical description of $P$ as a function of both aging time and aging temperature. In this earlier study, fitting of $P$ with respect to time was done before (upstream of) the fitting with respect to temperature.

Each of these eight properties was normalized with respect to the initial and peak-age property values pertinent to the age-hardening regime; these are termed $P$ (start) and $P($ peak), respectively, and were assumed to be independent of aging temperature. ${ }^{3}$ They are listed in Table 3.1. The fractional property change (or fraction transformed) as a function of some time variable, at constant temperature, is defined as

\footnotetext{
${ }^{3}$ Note that $P($ start $)$ corresponds to the start of the hardening reaction and is not necessarily equal to the as-quenched value $P(t=0)$, for example, when there is an initial transient softening effect before the onset of hardening. This distinction will be further elaborated later in this section.
} 


$$
f(\text { time })=\frac{P(\text { time })-P(\text { start })}{P(\text { peak })-P(\text { start })} .
$$

This equation can be rearranged to the form where $P($ time $)$ can be evaluated, given knowledge of f(time):

$$
P(\text { time })=P(\text { start })+f(\text { time }) \cdot(P(\text { peak })-P(\text { start })) .
$$

As is customary for the analyses of aging kinetics in metals and alloys, the aging time is assessed on a logarithmic scale, i.e.,

$$
x=\log _{10} \text { (aging time in minutes) }=\log (\text { time })
$$

The fraction transformed $f$ was fit to the time series data averages at each temperature using the following normalized (2-parameter) Boltzmann function:

$$
f(x)=1-\frac{1}{1+\exp \left(\frac{\left(x-x_{o}\right)}{d x}\right)} .
$$

The Boltzmann function in this format has two adjustable parameters $x_{o}$ and $d x^{4}$; these were adjusted by a commercially available software routine to optimize the fit for each log(time)series. The Boltzmann function has the following properties: $x=x_{0}$ is the $\log ($ time) at which $f=0.5 ; d x$ is related to the maximum slope of the curve: $\mathrm{d} f / \mathrm{d} x\left(\right.$ at $\left.x=x_{0}\right)=1 /(4 d x)$.

Reasonable $f(x)$ fits were obtained for the TE and 2YS properties, which were subjected to further analysis. Although the $\mathrm{HV}$ provided relatively poor fits for U-5.6Nb, it was further analyzed to enable comparison with other data sets [2002eck] — comparisons that are outside of the scope of this study. The remaining properties—1YS, 1YM, UTS, UE, and \%RA—had less-than-desirable fits and/or marginal utility in connection with a failure criterion and were not analyzed further.

The time-series fitting of the UE and TE at $100^{\circ} \mathrm{C}$ and $200^{\circ} \mathrm{C}$ was problematic because the UE and TE changed little (in relation to its own apparent scatter) over the time range studied, potentially introducing considerable errors into the fitting, which would flow downstream to errors in the apparent activation energy $Q$. At this point, the analyst intervened with an empirical solution where the time-series fit was nudged in the seemingly correct direction with the addition of a fictitious long-time data point where the UE and TE were expected to reach zero (equivalent to $f=1$ ). An example of this is given in Figure 3.1, located at $x=10.4$. Although such an intervention was not satisfying from the standpoint of avoiding bias, it was justified by the avoidance of much more significant errors downstream of this (in $\mathrm{Q}$ and the lifetime predictions), which were uncovered during trial attempts to fit the model without such an intervention.

The $\log$ (time)-series fits of the TE, 2YS, and HV were fed into an Arrhenius analysis to determine the temperature-dependencies of $f(x)$ by determining the scaling factor $Q$, the apparent activation energy of age-hardening. The determination of $Q$ is as follows: the $\log ($ time) values to

\footnotetext{
${ }^{4} x_{o}$ and $d x$ are referred to as $A$ and $B$, respectively, in [2007hac2]; be aware that $A$ and $B$ from the previous report were defined differently from the $A$ and $B$ in the present analysis (Section 3.3).
} 
reach a fixed value of $f$ are plotted against the reciprocal of the absolute temperature, and the best-fit line has a slope linearly related to the apparent activation energy $Q$ :

$$
\text { slope }=\frac{Q \cdot \log (e)}{R} \approx \frac{0.43429 Q}{R},
$$

where e is the base of the natural logarithm $(\approx 2.71828 \ldots)$ and $R$ is the universal gas constant. In the previous study, the $Q$ used for lifetime assessment was evaluated at the $\log ($ time) to reach $f=0.25$, with the understanding that somewhat different $Q$ values are obtained when it is evaluated at other values of $f$. This is a consequence of (1) the reaction not being strictly isokinetic (where $Q$ would be independent of $f$ ), combined with (2) the decision to fit $d x$ independently for each temperature (as opposed to forcing a common $d x$ value for all temperatures).

Tables 3.2 and 3.3 provide the best-fit values of $d x, x_{o}$, and $Q$.

Once $Q$ is determined, one can calculate the kinetically equivalent time to reach a given $f$ at some temperature $T_{2}$ if the time to reach that same value of $f$ is known at a reference temperature $T_{1}$ :

$$
\log \left(\text { time at } T_{2}\right)=\log \left(\text { time at } T_{1}\right)+\frac{Q \cdot \log (e)}{R}\left[\frac{1}{T_{2}}-\frac{1}{T_{1}}\right] \text {. }
$$

In Eq. 3.6, the temperatures $T_{1}$ and $T_{2}$ must be absolute (e.g., Kelvin). If $f(x)$ is completely defined for a reference temperature $T_{1}$, then the kinetic response $f(x)$ at some temperature $T_{2}$ is also completely defined through a mapping of the times using Eq. 3.6. In effect, the $f(x)$ curves will have identical shapes for all temperatures (provided that they use the same $d x$ value) when plotted on an $x=\log$ (time) abscissa. The curves are merely offset from one another along the abscissa by the additive factor, proportional to $Q$, the last term in Eq. 3.6. For the particular case of the Boltzmann model, this mapping is readily done by inserting the parameters $x_{o}\left(T_{2}\right)$ and $x_{o}\left(T_{1}\right)$ into Eq. 3.6, giving

$$
x_{o}\left(T_{2}\right)=x_{o}\left(T_{1}\right)+\frac{Q \cdot \log (e)}{R}\left[\frac{1}{T_{2}}-\frac{1}{T_{1}}\right] .
$$

Note that the Arrhenius temperature-scaling of the Boltzmann function in Eq. 3.7 only shifts $x_{0}$; there is no rescaling of $d x$, resulting in identically shaped curves offset only along the abscissa.

\subsubsection{Failure Criterion and Lifetime Prediction}

The quasi-static, uniaxial loading failure criterion [2007hac2], from which the lifetime is determined, is defined in terms of the property change relative to the time $=0$ condition $P(t=0)$,

$$
f^{\prime}(t)=\frac{P(\text { time })-P(t=0)}{P(\text { peak })-P(t=0)} .
$$

Failure is defined as taking place when this fractional change reaches a critical threshold:

$$
f^{\prime}(t=\text { lifetime })=f^{\prime}{ }_{\text {crit }} \text {. }
$$

A value of 0.25 was assigned to $f^{\prime}$ crit, which means that failure occurs when the property has changed by $25 \%$ relative to the $t=0$ condition. This can be equivalently stated as 


$$
P(t=\text { lifetime })=P_{\text {crit }}=P(t=0)+f_{\text {crit }}^{\prime}(P(\text { peak })-P(t=0)) .
$$

It should be noted that for the instance of historically manufactured components $P(t=0)$ would be $P(t=0, A M)$, while for the study here, it would be $P(t=0, M A)$. Regardless of whether the $t$ $=0$ condition is MA or AM, the $P(t=0)$ value is in general not the same as the $P($ start $)$ value, so $f^{\prime}(t)$ is not equivalent to $f(t)$. This complication arises from the observation of an apparent initial transient of softening, before the onset of hardening in the UE and TE. (It was much less noticeable in the HV, 1YM, 1YS, 2YS, UTS, and \%RA.) This softening is most noticeable in the $100^{\circ} \mathrm{C}$ and $200^{\circ} \mathrm{C}$ for both U-5.6Nb and U-7.7Nb, and can be perceived in Figure 3.1 as an initial upward deviation of the TE data points relative to the $P(t=0)$ line. The origin of this softening is unclear, though one plausible explanation is that thermally-induced relaxation of quenching stresses (MA condition) or machining stresses (AM condition) were imparted to the soft, easily strained martensitic microstructure at $\mathrm{t}=0$.

The most straightforward evaluation of the lifetime from this failure criterion would be to model $f^{\prime}$ (time). However, no attempt was made to model $f^{\prime}$ (time) of either U-Nb alloy, because this would ideally require explicit deconvolution of the softening portion of the aging curves vis-a-vis the hardening portion. At a minimum, a phenomenological fitting of the entire (nonmonotonic) aging curve without explicit consideration of the differences between softening and hardening regimes would require a more complex model with additional fitting parameters. Such an approach is not justified in view of the paucity of softening data. Instead, lifetime prediction was done by avoiding any explicit model of the softening part of the curve (apart from the $P$ (start) that results from softening) and simply determining the equivalent value of $f_{\text {crit }}$ that corresponds to $f_{\text {crit. }}^{\prime}$. The value of $f_{\text {crit }}$ is uniquely determined by the values of $P($ start $), P(t=0)$, $P$ (peak), and $f_{c r i t}^{\prime}$, according to the following relationship:

$$
P_{\text {crit }}=P(t=0)+f^{\prime}{ }_{\text {crit }}[P(\text { peak })-P(t=0)]=P(\text { start })+f_{\text {crit }}[P(\text { peak })-P(\text { start })] . \quad \text { Eq. } 3.11
$$

Rearranging to solve for $f_{c r i t}$ :

$$
f_{\text {crit }}=\frac{[P(t=0)-P(\text { start })]+f^{\prime}{ }_{\text {crit }}[P(\text { peak })-P(t=0)]}{[P(\text { peak })-P(\text { start }]} .
$$

For the special case of a property, such as TE, for which $P($ peak $)=0$, this simplifies to

$$
f_{\text {crit }}=1-\frac{\left(1-f^{\prime}{ }_{\text {crit }}\right) P(t=0)}{P(\text { start })} .
$$

Rearranging further,

$$
\left(1-f_{\text {crit }}\right) P(\text { start })=\left(1-f^{\prime}{ }_{\text {crit }}\right) P(t=0) .
$$

The equality in Eq. 3.14 is graphically depicted in Figure 3.2, which shows the TE for U-5.6Nb. On the basis of Eq. 3.13, the calculated values of $f_{\text {crit }}$ for TE are 0.3866 and 0.3404 for U-5.6Nb and U-7.7Nb, respectively. The corresponding $P_{\text {crit }}$ values were 0.1590 and 0.2572 , respectively. The failure criterion and threshold value $f_{\text {crit }}^{\prime}=0.25$, as described here, were accepted unchanged for the current analysis method described in Section 3.3.

For the lifetime determination, $T_{2}$ is the storage temperature of interest (e.g., $40^{\circ} \mathrm{C}$ ), whereas $T_{1}$ is the artificial aging temperature for which a Boltzmann time-series fit was determined $\left(100^{\circ} \mathrm{C}\right.$, $200^{\circ} \mathrm{C}, 250^{\circ} \mathrm{C}$, or $300^{\circ} \mathrm{C}$ ). Thus, four lifetime values were generated from the four values of $T_{1}$ 
and their fitted Boltzmann parameters, all of which used the common value of $Q$ for a given alloy. In the previous report, the spread of lifetimes (minimum-to-maximum) were listed. Given this scatter, it was decided that the conservative lifetime estimate to be reported should be the minimum of these four lifetime values. Table 3.4 provides all four calculated lifetimes (minimum one in bold), evaluated for the $f^{\prime} \geq 0.25$ failure criterion of TE.

Model fitting (parameter optimization) was carried out on Origin Pro software using its nonlinear least-squares fitting routine. No quantitative error assessment was made of this lifetime estimate or the contributing and propagating errors.

At the time of the previous report [2007hac2], no data independent of the data set used for the fitting of this Boltzmann-Arrhenius model were available for model validation; therefore this was not done.

\subsection{Current Analysis Method}

\subsubsection{Statistical Modeling Overview}

Seven of the eight age-sensitive properties listed in Section 3.2 were analyzed (all except \%RA) and fitted to an aging model in the current study; lifetimes continued to be estimated only on the basis of TE, as before. Table 3.5 lists the similarities and differences between the previous and current analysis methods. Given the statistical focus of the current analysis method, some background on the formalism and approach will now be provided.

The first goal of this statistical modeling is to describe the "population" of all possible outcomes (mechanical properties in this instance) of aged U-Nb. The second goal is to use this description to make predictions of future observations, which requires the use of a model. The population is described on the basis of a "sample" taken, preferably at random, from that population. It is the sample we observe, but the population that we seek to know. Because an infinite number of specimens cannot be aged and measured, the population will never be known in a perfectly precise fashion. The degree to which we can describe the population is derived from a characterization of scatter of the sample, which here is referred to as the model-fitting data.

The general form of a regression model is as follows:

$$
\mathrm{y}_{\mathrm{i}}=\mathrm{f}\left(\mathbf{X}_{\mathbf{i}} ; \boldsymbol{\theta}\right)+\varepsilon_{\mathrm{i}} ;
$$

where $y_{i}$ represents the response variable, $\boldsymbol{X}_{i}$ represents a vector of explanatory variables, and $\varepsilon_{i}$ represents the error term at the ith replicate data point, $i=(1,2, \ldots, n)$. The term $f(\boldsymbol{X} ; \boldsymbol{\theta})$ describes a function of the explanatory (independent) variables, represented by the vector $\boldsymbol{X}$, and the parameters to be fitted, represented by the vector $\boldsymbol{\theta}$. For the U-5.6Nb and U-7.7Nb data, the response (dependent) variables are the following properties: TE, UE, 1YS, 1YM, 2YS, UTS, and $\mathrm{HV}$, as well as the fractional property change for each of these properties. The explanatory variables are artificial aging time and temperature.

In situations where there aren’t first-principles models for complex physical mechanisms, such as those occurring in the materials studied in this report, the standard approach in statistical modeling is to develop empirical predictive models that that fit the data well and provide a reasonable manner for both interpolation and extrapolation. As a result, various functional forms and different parameterizations for $f(\boldsymbol{X} ; \boldsymbol{\theta})$ were considered. In previous studies [1976eck, 2002eck, 2007hac2], the use of an Avrami-Arrhenius model and a Boltzmann-Arrhenius model 
were explored. In this subsequent analysis, models similar in form to these, as well as a few other models, were examined.

Start and peak-age values for each property were the same as those used in the previous study [2007hac2], see Table 3.1. These upper and lower bounds are incorporated into the models considered in this report. For example, for fractional property change, the lower bound is 0 and the upper bound is 1 . Most of the models are presented in the form of a function where the property value increases as the explanatory variable, time, increases. For the properties UE and $\mathrm{TE}$, this relationship is reversed: the property value decreases with time.

\subsubsection{Model Evaluation}

Several models for the time-series response $y_{i}($ time $)$ or $y_{i}(x)$ at a constant temperature were examined and will presently be described.

The following model, similar in form to the Avrami model from the previous report, was considered:

$$
y_{i}=1-\exp \left(-A t_{i}{ }^{B}\right)+\varepsilon_{i} ;
$$

where $t$ is linear time. Eq. 3.8 has an alternate parameterization of the form

$$
y_{i}=1-\exp \left(-\exp \left(A-B t_{i}\right)\right)+\varepsilon_{i} .
$$

In both Equations. 3.16 and 3.17, $y_{i}$ represents the fractional property change $f$, as it varies from zero to one.

The general form of a Logistic model that was considered, similar to the Boltzmann model in the previous report (Eq. 3.4), is as follows:

$$
y_{i}=1-\frac{1}{\left[1+\exp \left(A-B x_{i}\right)\right]}+\varepsilon_{i} ;
$$

where the time-variable $x$ is defined in Eq. 3.3. Eq. 3.18 has alternate parameterizations of the form

$$
y_{i}=1-\frac{1}{\left[1+\exp \left(A\left(x_{i}-B\right)\right)\right]}+\varepsilon_{i}
$$

and

$$
y_{i}=\frac{U}{\left[1+\exp \left(A\left(x_{i}-B\right)\right)\right]}+\varepsilon_{i} ;
$$

where $U$ is a predetermined upper bound. In Eqs. 3.18 and 3.19, $y_{i}$ represents the fractional property change $f$, whereas it represents the absolute property value $P$ in Eq. 3.20, where $U$ has the same units as the property of interest, for example, MPa when the property is 1YS.

Another useful nonlinear model that was considered, the Gompertz model, is of the form

$$
y_{i}=1-\exp \left(-\exp \left(A-B x_{i}\right)\right)+\varepsilon_{i} ;
$$

with an alternate parameterization of the form 


$$
y_{i}=U \cdot \exp \left(-\exp \left(A-B x_{i}\right)\right)+\varepsilon_{i} ;
$$

where $U$ is a predetermined upper bound. In Eq. 3.21, $y_{i}$ represents the fractional property change $f(x)$, whereas it represents the absolute property value $P$ in Eq. 3.22, where $U$ has the same units as the property of interest.

Changing the parameterization of a model is simply a way of improving the fitting and predictive behavior of the nonlinear model. Better behaved models produce better parameter estimates. For more information on various forms of nonlinear models and their parameterizations, see Ratkowsky [1990rat]. Note that in this context, alternate parameterizations are those belonging to a particular class of model, and are not necessarily symbolically equivalent, in the sense that a simple substitution of variables will transform one equation to its alternate. The special case of true symbolic equivalency does occur, however, between Eqs. 3.4 (Boltzmann), 3.18 (Logistic), and 3.19 (Logistic).

Model fitting (parameter optimization) and related statistical analyses were carried out on S-Plus software licensed by Insightful Corp. See Venables and Ripley [1994ven] for details on the software code. The primary function for fitting a nonlinear regression model in S-plus is $n l s$. The inputs provided to this routine include a nonlinear model formula expressing the model, an optional data frame as the reference point for the variables in the model, a list of starting values for the parameters to be estimated, an optional argument allowing control of some features of the iterative procedure, an optional argument specifying the iterative procedure, and an argument allowing trace information to be printed.

Given the general form of a nonlinear regression model as in Eq. 3.15, the following provides a general outline of the fitting algorithm:

1. Near an initial point, the solution locus is approximated by its tangent plane.

2. The observation vector $\boldsymbol{y}$ is projected onto the tangent to give a new coefficient vector, $\tilde{\boldsymbol{\theta}}$.

3. The tangent plane is calculated at the new coefficient vector, $\boldsymbol{\theta}$, and the procedure continues until convergence occurs.

Trial fits with the three models and their various parameterizations (Eqs. 3.16-3.22) were carried out. The residual standard errors (RSEs) for the various fits were compared. This summary statistic represents the variation of the actual data points around the mean fitted values from the model. Plots of residuals from the model were used to look for a constant variance, independence of successive noise terms, and also assess model adequacy. If the model provides an adequate fit to the data, then the residuals from the model plotted against the quartiles of a standard normal distribution should fall along a straight line. Normal probability plots of the residuals are provided in Appendix 3, along with profile plots of the residual sum-of-squares (RSS) function to check for linearity in the parameters. The goodness of fit of a nonlinear model can be assessed by the degree of linearity of the parameter estimates (i.e., linearity implies that the model is stable with respect to perturbations in $A, B$, and $Q$ ).

Based on various statistical tests of goodness of fit as well as practicality and understanding of the model, it was determined that the best fit to the data was provided by the Logistic model using the second parameterization (Eq. 3.19). This is the model that will be presented and discussed in the rest of the report. Note that this is equivalent to the Boltzmann model (Eq. 3.4) 
with the following substitutions: $A=1 / d x$ and $B=x_{0}$. Therefore, the Logistic model can be considered as a symbolically equivalent parameterization of the Boltzmann model.

To summarize, the outcome of this initial survey of empirical models and variables recommended the following for further use, which were employed for the remainder of this study:

1. Logistic model, second parameterization (Eq. 3.19) for time-series fits.

2. $x=\log$ (time) (Eq. 3.3) as the independent variable for the Logistic model.

3. Fractional transformed $f$ (Eq. 3.1) as the dependent variable for the Logistic model.

4. Arrhenius model (Eq. 3.6) for temperature-series fits.

This Logistic-Arrhenius model can be considered semiempirical (instead of purely empirical) because it retains the Arrhenius temperature dependence, which can in some instances, provide scientific insight, from the value of $Q$, into the physical aging mechanism(s).

\subsubsection{Model Fitting}

The initial parameter-fitting approach was similar to that used in the previous study: the timeseries data for the four aging temperatures were fit independent of one another and before (upstream of) fitting these as a temperature series. From these four time-series fits (one for each temperature), the point $x$ where each of the curves crossed a predetermined threshold line, $f=0.25$, was obtained, and a straight line was fit to these points when plotted against $1 / T$. Eq. 3.5 then was used to provide an estimate of the activation energy, $Q$. This regression involved one data point with high leverage (the point from the fit to the $100^{\circ} \mathrm{C}$ data that had the most variability). In order to find a model that would be less sensitive to the influence of one data point, an alternate method for fitting the parameters and estimating $Q$ was sought.

In this alternate method, the data from all four accelerated aging temperatures were combined into one data set for analysis by collapsing the data from separate temperatures onto a universal aging-response plot. To do this, the nonlinear Logistic model (Eq. 3.19) for fractional property change (Eq. 3.1) is couched in terms of a modified, universal time variable $x^{\prime}$;

$$
f=1-\frac{1}{\left[1+\exp \left(A\left(x^{\prime}-B\right)\right)\right]}+\varepsilon .
$$

The times from various aging temperatures $T_{1}$ were rescaled to equivalent $\log ($ times $) x^{\prime}$ at a reference temperature $T_{2}$ through the Arrhenius relationship, where the temperatures are in Kelvin:

$$
x^{\prime}=\log \left(\text { time at } T_{2}\right)=\log \left(\text { time at } T_{1}\right)+\frac{Q \cdot \log (e)}{R}\left[\frac{1}{T_{2}}-\frac{1}{T_{1}}\right] \text {. }
$$

The analysis is independent of the specific choice of $T_{2}$; for convenience, $T_{2}$ was set at $300^{\circ} \mathrm{C}=573.15 \mathrm{~K}$. The error term, $\varepsilon$, is distributed as a Normal random variable, with mean, 0 , and variance, 1 , written as $\varepsilon \sim \mathrm{N}(0,1)$.

Note that the Eq. 3.24 comes from the assumption of Arrhenius extrapolation that serves to shift the entire aging-response, fraction-transformed curve from one temperature to another while preserving the shape of the curve on a log time scale. 
With all of the data collapsed onto a single universal aging-response plot (through Eqs. 3.23 and 3.24), the effects of both time and temperature could be assessed simultaneously rather than sequentially, resulting in improved estimates for the Logistic time-series model parameters $A$ and $B$, and for the activation energy, $Q$. The nonlinear least-squares fitting of $A, B$, and $Q$ was subjected to the following constraints. The parameter $A$ had the same value for all temperatures, in effect ensuring shape similarity among fitted and predicted curves for different temperatures. This constraint allowed the data to be collapsed onto the universal plot, and in effect forced an isokinetic description (where $Q$ is independent of the $f$ at which it is evaluated). Furthermore, $A$, $B$, and $Q$ were further constrained to conform to a "perfect-Arrhenius" description, where any best-fit line on an Arrhenius plot passes through the model-fitted point for each temperature exactly, making it a "perfect-fit" line.

The parameter estimates for each property are given in Tables 3.6-3.19.

\subsubsection{Model Predictions and Statistical Intervals}

Combining Eqs. 3.23 and 3.24 allows the fraction-transformed time-response for any given property to be calculated as follows:

$$
f(x)=1-\frac{1}{\left[1+\exp \left(A\left(x+\frac{Q \cdot \log (e)}{R}\left[\frac{1}{573.15 K}-\frac{1}{T}\right]-B\right)\right)\right]}+\varepsilon_{i} .
$$

To compute predicted $f(x)$, replace the parameters $A, B$, and $Q$ in Eq. 3.17 with their estimates, replace the $x$ with the $\log$ (time in minutes), and replace $T$ with the temperature in Kelvin. For a given property and alloy, the estimated parameters for the $f(x)$ and $P(x)$ models are the same, because fractional property change is a linear transformation of the property value:

$$
P(x)=P(\text { start })+f(x) \cdot(P(\text { peak })-P(\text { start })) .
$$

The property response at any given aging time and temperature can be computed by substituting Eq. 3.25 into 3.26. The method for computing approximate confidence intervals (CIs) and prediction intervals (PIs) [1982mee, 1991hah] are described in Appendix 4. A CI indicates the probabilistic degree of scatter (for example, at the 95\% level) of the true predicted value (the population mean) on the basis of the scatter in the data- $n$ observations - used in model-fitting ("the sample," a subset of the population). The $C I$, by accounting for the uncertainty in the fitted model parameters $A, B$, and $Q$, will contain the mean of the sampled population, in the long run, at the specified level of confidence. The $C I$ is compared against the $n$ data points used to fit the model.

A PI, by contrast, indicates the degree of scatter expected for $n v$ future ${ }^{5}$ replicate measurements (or, on a slightly modified formula, their average) given the model fit from the $n$ observations. In this report, $n v$ refers to the number of validation specimens, because data from such specimens are what will be compared with the PI; standard reference works on this topic call this " $\mathrm{k}$ " [1982mee, 1991hah] or "m" [1974net]. The PI will contain the value(s) of these $n v$ future observations, in the long run, at the specified level of confidence. The PI is by definition wider than a $C I$ at a given value of $x=\log ($ time), because it includes the CI's scatter in the mean value

\footnotetext{
${ }^{5}$ The computation of a PI is the same regardless of whether the $x=\log ($ time) of the future age being evaluated lies inside or outside the time-temperature bounds of the model-fitting data.
} 
position plus an additional source of scatter, that of the random variation in the future result about the mean. Figure 3.4 in Neter and Wasserman demonstrates this point [1974net].

The lifetime for each alloy at a variety of aging (storage) temperatures was evaluated with the same $f^{\prime} \geq f^{\prime}{ }_{\text {crit }}=0.25$ criterion for TE as described in Section 3.2; see particularly Eqs. 3.8-3.14. Because of the "perfect-Arrhenius" constraint placed on the fitting of $A, B$, and $Q$, only a single mean value of the predicted lifetime resulted. The error assessment associated with this fitting provided 95\% confidence intervals about this mean value.

\section{RESULTS AND DISCUSSION}

\subsection{Model Fitting-General Comments}

\subsubsection{Quality of Fitting}

Seven age-sensitive properties were fitted to the same three-parameter $(A, B$, and $Q)$ LogisticArrhenius model. The optimized (fitted) models will be evaluated on the basis of three criteria: goodnesses of fit, robustness of fit, and symmetry of fit. These three evaluations of fitting quality will be preceded by several remarks about the generation of universal aging response plots that are useful for these evaluations.

Universal aging response plots for all model fits are shown in Figures 4.1-4.14. The experimental data and fitted curve for all temperatures are collapsed onto the same time axis by scaling them to a common reference temperature, according to Eqs. 3.23-3.25. For these plots, $300^{\circ} \mathrm{C}$ was chosen as the reference temperature, though this choice is done only for convenience: any temperature can be selected. This is essentially the same "view" that the current modelfitting routine had, where it adjusted the $A, B$, and $Q$ parameters to give the best fit Logistic curve to the data thus displayed. It is helpful at this point to recall the geometric meanings of the parameters and their specific influences on the Logistic-Arrhenius model curve shapes:

- $A$ is equal to four times the slope at $x=B$ (and also where $f=0.5$ ); a higher value of $A$ results in a more steeply sloped fitted curve.

- $B$ is the $x$-value at which $f=0.5$; a higher value of $B$ uniformly shifts the fitted curve to the right.

- $Q$ scales the aging times at the actual aging temperature to equivalent aging times at the reference temperature. Data points from each temperature shift as a bloc when $Q$ is changed; furthermore, the shifts of all temperature blocs are correlated, being uniquely determined by the value of $Q$.

Note that the $40^{\circ} \mathrm{C}, 65^{\circ} \mathrm{C}$, and $90^{\circ} \mathrm{C}$ data points in the U-5.6Nb universal plots are from the banded U-6Nb, not the nonbanded U-5.6Nb; they can be plotted on the same axes when the $\mathrm{y}$-axis is $f$ because they use the same $A, B$, and $Q$ parameters. Data from $\mathrm{U}-5.6 \mathrm{Nb}$ and $\mathrm{U}-6 \mathrm{Nb}$ cannot, however, be plotted on the same axes when the y-axis is the absolute property because their values of $P$ (start) and $P$ (peak) can differ (Table 3.1.)

The shifting (offsetting) of the time axis for the data points from other temperatures is not shown (as it is, for example, in Eckelmeyer's universal curve of U-Ti aging-Figure 7 of [1976eck]), but can be conceptually understood as follows. The simplest way is to take advantage of the fact the earliest-time replicate point for each temperature series is at 10 minutes. Find the $x=1$ (i.e., the 10 minute) point for $300^{\circ} \mathrm{C}$. Now shift (without distorting) the visible log(time) axis for 
$300^{\circ} \mathrm{C}$ to the left until that point lines up over the 10-minute data point for the (lower) temperature of interest. The axis shifted accurately represents all the data points of this other temperature. (The vertical axis, $f$, required no rescaling.)

Specific comments will now be made on the three quality-of-fitting metrics: goodnesses of fit, robustness of fit, and symmetry of fit. This information is summarized in Tables 4.1 and 4.2.

The first evaluation of the model, the goodness of model fit with respect to the experimental data, is best quantified by a scalar value, the RSE, which represents the variation of the observed values around the predicted values ${ }^{6}$ from the model. Indeed, the "best-fit" or optimized model parameters are defined as those that give the minimum RSE value. The RSE is defined as

$$
R S E=\sqrt{\sum_{i=1}^{n} \frac{\left(y_{i}-\text { pred }_{i}\right)^{2}}{(n-p)}} .
$$

In Eq. 4.1, $y_{i}$ represents an observed measurement, pred $_{i}$ represents a predicted value from the nonlinear model for the $i$ th replicate data point; these can be either absolute property values or their normalized value, $f$. The total number of replicate points used in the fitting process is $n$, and $p$ is the number of parameters in the model (for this model $p$ is always 3: $A, B$, and $Q$ ). The difference $n-p$ is called the degrees of freedom $(d f)$ of the fit.

A lower value of the RSE indicates a better fit of the model to the data. Values of the RSE and ancillary quantities are provided in Table 4.3. The following trends are observed when comparing the fractional RSE values:

1. 2YS and HV showed the best fits in both alloys. For 2YS, this is a similar outcome as the previous study [2007hac2]. For HV, this outcome is an improvement, especially for U-5.6Nb.

2. 1YM fit the least well in both alloys.

3. TE and UE fit moderately well in both alloys.

4. 1YS fit very well in U-5.6Nb but fit only moderately well in U-7.7Nb.

5. UTS fit very well in U-7.7Nb but fit only moderately well in U-5.6Nb.

The second evaluation, robustness of fitting of the individual parameters $(A, B$, and $Q)$, is done by RSS testing, the details of which are in Appendix 3. Relatively low robustness in one or more parameters were identified for $1 \mathrm{YM}$ in U-5.6Nb and TE, UE, 1YM, and HV for U-7.7Nb. Note that poor $R S S$ test results do not necessarily correlate to the poor (i.e., high) RSE value, or viceversa; however, a poor showing in either metric casts greater doubt on the validity of the model for the particular property in question.

The third evaluation, symmetry of fitting, refers to how evenly the data points from specific aging temperatures are distributed about the fitted curve. A markedly uneven distribution among the points for a given temperature (or span of time) is a statistical sign that the model may not be applicable to this temperature (or span of time). Focusing on the $100^{\circ} \mathrm{C}$ data points in the universal plots (Figures 4.1-4.14) reveals an important difference between the two alloys. For all of the properties, most of the observed $100^{\circ} \mathrm{C}$ data points for $\mathrm{U}-7.7 \mathrm{Nb}$ alloy consistently lie

\footnotetext{
${ }^{6}$ In this report, the terms "predicted values," "mean fitted values,” and "model predictions” are used interchangeably.
} 
above the predicted curve; however, this trend does not occur for the U-5.6Nb alloy data points, which are distributed more evenly. Essentially, what this implies is that there is an underlying physical consistency in the relationship between the curves at all of the accelerated aging temperatures for the U-5.6Nb alloy data, but not for the U-7.7Nb alloy data. For the U-7.7Nb, there is only consistency between the $200^{\circ} \mathrm{C}, 250^{\circ} \mathrm{C}$, and $300^{\circ} \mathrm{C}$ curves and below $200^{\circ} \mathrm{C}$ there is a change, perhaps attributable to a change in the physical aging mechanism. By contrast, the results of this study provide no statistical evidence that there is a change in aging mechanism at lower temperatures for U-5.6Nb.

To summarize, both alloys show reasonable goodnesses of fit, which vary from property to property. However, the U-5.6Nb models are more robust and symmetric, and therefore are expected to have better predictive power than those of $\mathrm{U}-7.7 \mathrm{Nb}$, especially at the lower aging temperatures $\left(<100^{\circ} \mathrm{C}\right)$ of interest.

\subsubsection{Comparison of Parameters and Aging Responses}

The values of fitted parameters from the models for all properties and alloys are summarized in Tables $4.4(Q), 4.5(A)$, and $4.6(B)$. The values from the present study can be compared with those of the previous study for the TE, 2YS, and HV properties. For the most important property, TE, the new values of the activation energy $Q$ are both higher than the old values: $10 \%$ higher (to $32 \mathrm{kcal} / \mathrm{mol}$ ) for $U-5.6 \mathrm{Nb}$ and $50 \%$ higher (to $39 \mathrm{kcal} / \mathrm{mol}$ ) for $\mathrm{U}-7.7 \mathrm{Nb}$. The old and new $A$ and $B$ values for TE are almost identical for U-5.6Nb, though they differ significantly for $\mathrm{U}-7.7 \mathrm{Nb}$. This finding suggests that the old and new lifetimes will be similar for U-5.6Nb and less similar for U-7.7Nb. The differences between the old and new modeling approaches come into greater relief when comparing their values for $2 \mathrm{YS}$ in $\mathrm{U}-7.7 \mathrm{Nb}$ and $\mathrm{HV}$ for both alloys. In particular, the old and new $\mathrm{HV}$ values for U-5.6Nb are dramatically different, going from 116 $\mathrm{kcal} / \mathrm{mol}$ to $28 \mathrm{kcal} / \mathrm{mol}$, and while at the same time shifting $B$ (the $\log ($ time) at which $f=0.5$ ) forward in time by six orders of magnitude (in linear time), from 9.4 to 3.4. This extreme example highlights the influence that points of high leverage $\left(100^{\circ} \mathrm{C}\right.$ data in this instance) have when examined through two different analysis methods.

Turning now to a comparison only among the parameters fitted for this study, the following generalizations can be made. The activation energy $Q$ will be examined first, as follows:

1. $Q(\mathrm{TE})$ in both alloys is close to that for Nb volume diffusion in $\gamma-\mathrm{U}$, $\mathrm{Q}_{\text {diff }}=32.6 \mathrm{kcal} / \mathrm{mol}$, marginally so for U-7.7Nb, which is $20 \%$ higher. The similarities between $Q(T E)$ and $Q_{\text {diff }}$ are not inconsistent with previous studies [1976eck, 2002eck, 2007hac2]. In light of the most recent atom probe experiments [2008cla] (which indicate that $\mathrm{Nb}$ diffusion is not even relevant at $200^{\circ} \mathrm{C}$ and below) we note that the apparent agreement between any measured $Q$ and $Q_{\text {diff }}$ could merely be coincidental.

2. $Q(\mathrm{TE})$ for $\mathrm{U}-7.7 \mathrm{Nb}$ is higher than that of $\mathrm{U}-5.6 \mathrm{Nb}$, which is opposite the trend of previous studies [2002eck, 2007hac2]. However, Q(HV) for U-7.7Nb is lower than that of U-5.6Nb, which is the same trend observed before.

3. $1 Y S$ and $1 Y M$, have consistently low $Q$, roughly one-third to two-thirds that of TE.

4. $Q(\mathrm{UE})$ and $Q(\mathrm{TE})$ are nearly identical for $\mathrm{U}-5.6 \mathrm{Nb}$, and differ by $20 \%$ for $\mathrm{U}-7.7 \mathrm{Nb}$. Such similarities are consistent with the fact that UE is usually the major contributor to the TE, especially at lesser degrees of aging when reasonably high ductility obtains. 
5. Q(UTS) values for both alloys are within $10 \%$ of one another.

In summary, activation energies for the properties spanned 14-52 kcal/mol. Given the aforementioned difficulties interpreting the Q values for TE (point \#1), further comment on specific physical mechanisms responsible for the $\mathrm{Q}$ values from other properties is not warranted at this time.

Comparison of the impact of differing $A$ and $B$ values among the seven properties is best done graphically. The variation in the aging response of the seven properties is highlighted in Figures 4.15 and 4.16. The fractional property change is used to enable direct comparison among the properties. Two representative temperatures are plotted; $200^{\circ} \mathrm{C}$ is a typical accelerated aging temperature, and $40^{\circ} \mathrm{C}$ is a representative storage temperature.

At $200^{\circ} \mathrm{C}$ (Figure 4.15 ), the seven property responses are more tightly grouped with respect to $\log$ (time) in U-5.6Nb than in U-7.7Nb. This is consistent with the smaller range of $B$ parameter values (Table 4.6) in $\mathrm{U}-5.6 \mathrm{Nb}(+0.9955$ to +2.5672$)$ relative to those corresponding to $\mathrm{U}-7.7 \mathrm{Nb}$ $(-0.6659$ to +4.1543$)$. The maximum slope of the curve, proportional to parameter $A$ (Table 4.5$)$, shows less scatter in U-5.6Nb than in U-7.7Nb. For both alloys, TE, UE, and 2YS respond earlier in time at $200^{\circ} \mathrm{C}$, but the UTS response lags in time.

The differences between properties and alloys become much more pronounced when comparing the predictions at $40^{\circ} \mathrm{C}$ (Figure 4.16), where the differences in the activation energy $Q$ (Table 4.4) are the most significant contributors to the splitting of the curves relative to higher temperatures, such as $200^{\circ} \mathrm{C}$ (Figure 4.15). Eight to nine orders of magnitude time difference separate the response of the earliest-changing property (1YM) from the latest-developing one (UTS). The properties in both alloys (except for the UE, as will be noted) can be grouped into three categories:

1. Early-time responders at $40^{\circ} \mathrm{C}: 1 \mathrm{YM}$ and $1 \mathrm{YS}$

2. Middle-time responders at $40^{\circ} \mathrm{C}$ : TE, HV, $2 \mathrm{YS}$, and UE (U-5.6Nb only)

3. Late-time responders at $40^{\circ} \mathrm{C}$ : UTS and UE (U-7.7Nb only)

Of all seven properties examined, one should trust the 1YM predictions the least because they had the poorest model fits (i.e., with the highest RSE, Table 4.3) for both alloys. The diverging behavior of the UE between the two alloys is somewhat puzzling. In general, one would expect UE and TE to follow the same pattern, because UE is a major component of TE. The superficial cause of the UE lagging so far behind in U-7.7Nb is its very high activation energy, $52 \mathrm{kcal} / \mathrm{mol}$ (the highest of any $Q$ fitted in this study), since curves for a given property will be spread out more at lower temperatures with a higher value of $Q$ than a lower value (Eqs. 3.6 and 3.7). A more fundamental physical explanation for why the data for U-7.7Nb resulted in such a high value of $Q(\mathrm{UE})$ was not readily apparent.

\subsubsection{Modeling Assumptions}

The previous and current modeling process (summarized in Table 3.5) gave similar outcomes for all three parameters for TE and 2YS in U-5.6Nb, and for none of the TE parameters for U-7.7Nb. This provides added assurance in the robustness of the TE and 2YS models in U-5.6Nb, while casting some doubt on the robustness of the TE model in U-7.7Nb. This finding is consistent with the RSS analysis in Appendix 3. Vastly different results were obtained from the two approaches for the case of the least well-behaved property, $\mathrm{HV}$ in U-5.6Nb, highlighting the 
sensitivity of the modeling approach for property responses that appear to be poorly behaved, especially with regards to high-leverage data (i.e., $100^{\circ} \mathrm{C}$ ).

The validity of each of the models depends on the following assumptions:

1. The model is able to explain or represent all of the reactions or processes taking place within the experiment.

2. Related to \#1 is the further assumption that such aging-related property changes, which are indirect measures of aging, bear a meaningful, if not unique correlation with the time progression of the underlying physical processes (e.g., diffusional nucleation and growth of precipitates) that are ultimately responsible for such property changes.

3. The estimated start value and peak value for each property are accurate and are valid for all aging and storage temperatures (Table 3.1).

4. The dominant source of error in the measured properties is due to the intrinsic material variability, and all other sources of error are either negligible or are incorporated in the measure of intrinsic material variability (Appendix 1).

5. There are no gross systematic errors present in the measurements. A systematic error is a type of error that remains constant over a series of experiments and would result in an offset, or bias, between the estimated measurements and the "true" measurement values (Appendix 1).

6. The shape of the response curve is the same at each of the accelerated aging temperatures. In terms of the Logistic (Boltzmann) model, this means that $A(=1 / d x)$ is the same for all aging temperatures.

7. A linear relationship, on a log(time) scale, exists between the curves at different temperatures, defined by the Arrhenius formalism developed for describing the kinetics of thermally activated processes.

8. The reactions and processes taking place at each of the accelerated aging temperatures are the same and are representative of the temperatures of interest (i.e., near-ambient storage temperatures).

9. Aging in banded U-6Nb (6.3 wt\% Nb measured composition) can be represented by the same $A, B$, and $Q$ parameters fitted to the nonbanded $U-5.6 N b$ data.

Assumptions 1 and 2 are the most critical, and the most subject to uncertainty given the current lack of maturity regarding the fundamental scientific understanding and the related paucity of predictive power of aging models in general, and U-Nb alloys in particular. Such concerns were reiterated in a recent study of aging mechanisms in U-5.6Nb [2008cla]. Significantly more fundamental mechanistic studies would be required to critically evaluate these assumptions in a more satisfactory manner.

Assumption 3 was accepted as-is in this study, e.g., no error bars were assigned to the $P$ (start) and $P$ (peak) values. Adding error bars and/or refining these values are recommended for examination in future studies, especially with regard to the initial softening transient that is observed before the onset of phenomenological age-hardening in U-Nb alloys. Assumptions 4 and 5 were examined and deemed to be reasonably valid. Assumptions 6 and 7 were implicitly 
tested through the examination of the goodness of fits in Sections 4.2 through 4.8, with results that varied from good to poor, depending on the property and alloy.

Assumptions 8 and 9 were tested with the banded $\mathrm{U}-6 \mathrm{Nb}$ validation data at $40^{\circ} \mathrm{C}, 65^{\circ} \mathrm{C}$, and $90^{\circ} \mathrm{C}$ and fared well, as will be described in Section 4.3. However, the fitting symmetry considerations in Section 4.1.1 call into question the validity of Assumption 8 to U-7.7Nb, because its behavior for all properties at $100^{\circ} \mathrm{C}$ (and possibly lower temperatures still) appears to be different than at $200^{\circ} \mathrm{C}, 250^{\circ} \mathrm{C}$, and $300^{\circ} \mathrm{C}$.

\subsection{Model Fitting-Specific Properties}

Sections 4.2.1-4.2.7 describe the model fitting results specific to each of the properties. Note that every replicate data point is plotted on the graphs. Both $f(x)$ and $P(x)$ graphs are given for each property and alloy; these are linearly-scaled (along the $y$-axis) versions of each other according to Eqs. 3.1 and 3.2. These sections also provide property-response curves with $95 \%$ confidence interval bounds at three representative temperatures of stockpile interest: $30^{\circ} \mathrm{C}, 40^{\circ} \mathrm{C}$, and $50^{\circ} \mathrm{C}$.

\subsubsection{Total Elongation}

Figures 4.17 and 4.18 show the fitted model results for TE. For both alloys, the predicted curves at $300^{\circ} \mathrm{C}$ don't seem to be steep enough to fit the points at the largest age. As observed for the other properties, it appears that the data points for $\mathrm{U}-5.6 \mathrm{Nb}$ at $100^{\circ} \mathrm{C}$ are scattered fairly evenly about the predicted curve, but for the U-7.7Nb data, most of the points lie above the predicted curve (when $f$ is the y-axis). Examination of the universal aging curves (Figures 4.1 and 4.2) highlights the considerable scatter of the $100^{\circ} \mathrm{C}$ data in both alloys.

\subsubsection{Uniform Elongation}

Figures 4.19 and 4.20 show the fitted model results for UE. The predicted curves seem to fit reasonably well for the $\mathrm{U}-5.6 \mathrm{Nb}$ data, given that there is a lot of scatter in the data. But for the $\mathrm{U}-7.7 \mathrm{Nb}$ data, all of the $100^{\circ} \mathrm{C}$ data points lie above the predicted curve (when $f$ is the $\mathrm{y}$-axis), indicating that the relationship between the curves, as defined by the activation energy, may not be valid at temperatures lower than $200^{\circ} \mathrm{C}$. In fact, the UE for U-7.7Nb yielded the highest Q, 52 $\mathrm{kcal} / \mathrm{mol}$, of any of the property-alloy combinations in this study. Given the skewed scatter of the points noted previously, it may be that two (or possibly more) aging regimes transpire: one with $Q>52 \mathrm{kcal} / \mathrm{mol}$ over $200^{\circ} \mathrm{C}-300^{\circ} \mathrm{C}$, and the other with $Q<52 \mathrm{kcal} / \mathrm{mol}$ at $<200^{\circ} \mathrm{C}$. Examination of the universal aging curves (Figures 4.3 and 4.4) highlights the considerable scatter of the $100^{\circ} \mathrm{C}$ data in both alloys, though the magnitude of scatter is somewhat less than for TE, an observation consistent with the contribution of stochastic necking-and-failure processes to TE, but not UE.

\subsubsection{First-Yield Strength}

Figures 4.21 and 4.22 show the fitted model results for 1YS. For this property, the slopes of the predicted curves are steeper than for most of the other properties, and the curves at the different aging temperatures are closer together, indicative of relatively low activation energies for this property compared to that of the other properties. The model appears to provide a better fit to the $\mathrm{U}-5.6 \mathrm{Nb}$ alloy data than to the U-7.7Nb alloy data, where the data points in the latter at both $300^{\circ} \mathrm{C}$ and $100^{\circ} \mathrm{C}$ don't seem to fall along their representative curves. Examination of the universal aging curves (Figures 4.5 and 4.6) highlights the relative lack scatter among all data in both alloys. 


\subsubsection{First-Yield Modulus}

Figures 4.23 and 4.24 show the fitted model results for 1YM. The estimated activation energies are the smallest of all the properties for both $\mathrm{U}-5.6 \mathrm{Nb}$ and $\mathrm{U}-7.7 \mathrm{Nb}$. In addition, the scatter in the data points at all temperatures is relatively large (see also the universal aging curves in Figures 4.7 and 4.8), making it appear that the predicted curves don’t adequately fit the data.

\subsubsection{Second-Yield Strength}

Figures 4.25 and 4.26 show the fitted model results for $2 \mathrm{YS}$. The predicted curves at the aging temperatures appear to fit better for the U-5.6Nb alloy data than for the U-7.7Nb alloy data. The fits for both alloys are marginal at $100^{\circ} \mathrm{C}$ : for $\mathrm{U}-5.6 \mathrm{Nb}$, most of the data points fall below the curve; therefore, for the U-7.7Nb data, all of the points fall above the curve. Statistical models are expected to fit data in an unbiased way. It is expected that the data points would be randomly scattered about the fitted curve. In other words, approximately half of the data points would be expected to lie above a population-wide model fit, and the other half expected to lie below. The fact that most of the points at $100^{\circ} \mathrm{C}$ lie on one side of the predicted curve could be an indication that there is a fundamental difference between the reactions at the higher temperatures and the reactions at $100^{\circ} \mathrm{C}$ that is not adequately captured by a single value of the activation energy over $100^{\circ} \mathrm{C}-300^{\circ} \mathrm{C}$. In other words, an Arrhenius relationship with a single value of $Q$ obtained over $200^{\circ} \mathrm{C}-300^{\circ} \mathrm{C}$ may not hold when applied to curves at temperatures below $200^{\circ} \mathrm{C}$. Examination of the universal aging curves (Figures 4.9 and 4.10) highlights the relative lack scatter among all data in both alloys.

\subsubsection{Ultimate Tensile Strength}

Figures 4.27 and 4.28 show the fitted model results for UTS. For the U-7.7Nb data, all the predicted curves seem to fit at the three higher temperatures, but at $100^{\circ} \mathrm{C}$, all of the data falls above the curve. According to the estimated value for $Q$, it is expected that these measured values would be smaller than they actually are. In effect, the data all falling above the predicted curve at $100^{\circ} \mathrm{C}$ could indicate that there is something different about the experiment at $100{ }^{\circ} \mathrm{C}$ that is not accounted for in the model. For the U-5.6Nb data, the curves seem to fit the average trend reasonably well, partly because of the large scatter among the data points. Examination of the universal aging curves (Figures 4.11 and 4.12) highlights the relatively high scatter in $\mathrm{U}-5.6 \mathrm{Nb}$ and the relatively low scatter in U-7.7Nb.

\subsubsection{Vickers Hardness}

Figures 4.29 and 4.30 show the fitted model results for HV. For the U-5.6Nb alloy, the predicted curves seem to fit well at all of the temperatures except for $200^{\circ} \mathrm{C}$, where all of the points fall above the predicted curve. For the U-7.7Nb alloy, all of the points fall above the predicted curve at $100^{\circ} \mathrm{C}$. Examination of the universal aging curves (Figures 4.13 and 4.14 ) highlights the relative lack scatter among all data in both alloys.

\subsection{Model Validation}

Validation data and predictions from the models are listed in Tables 4.7-4.13. The entire validation aging matrix is represented; data for only a few of the ages were available at the time these predictions were made; the ages without experimental data values are either planned or already pending. All of the validation ages require extrapolation of the aging model.

Extrapolations outside of the time-temperature domain of the model fitting provides a more severe test of the model than validation experiments that would involve model interpolations. In 
the case of U-5.6Nb and U-7.7Nb, only extrapolation to longer times is done, because the aging temperatures are a subset $\left(100^{\circ} \mathrm{C}, 200^{\circ} \mathrm{C}\right.$, and $\left.300^{\circ} \mathrm{C}\right)$ of those used for model fitting. In the case of banded U-6Nb, combined extrapolation to both longer times and lower temperatures is required with respect to the times and temperatures employed for model fitting, providing the more stringent validation test.

Validation plots for U-5.6Nb, U-7.7Nb, and U-6Nb are provided in Figures 4.31, 4.32-4.38, and 4.39-4.45, respectively. The validation data are also plotted in the universal aging response plots (Figures 4.1-4.14), which aid in the visualization of the validation data in the context of the overall model fit. All seven properties were measured in U-7.7Nb and U-6Nb, while only hardness was measured in U-5.6Nb because of specimen limitations for the ages documented in this study (tensile data for U-5.6Nb are planned for longer ages, though).

The model predictions listed in Tables 4.7-4.13 include mean values, 95\% CI, and 95\% prediction interval (PI) bounds. The PI bounds define the relevant scatter band of the model predictions for the validation plots. Appendix 4 explains the definition and computation of confidence interval $(C I)$ and $P I$ bounds; they are also briefly described in Section 3.3.4. If a validation data point were to fall outside of the prediction interval bounds, then one could conclude either that the sample is different from the population from which the model was obtained, or that the model does not adequately represent the population. The validation data points for all the tensile properties are the averages of more than one measurement: $n v=2$ in the case of $\mathrm{U}-7.7 \mathrm{Nb}$ and $n v=3$ in the case of U-6Nb. (Recall that data points from HV scans from a given aged coupon are considered to be a single replicate, so $n v=1$ for hardness in all alloys.) The PI bounds were calculated on the assumption that they will be compared with these averages, given their associated $n v$ value. $^{7}$

The HV validation points in U-5.6Nb (Figure 4.31) show good agreement with prediction. The $100^{\circ} \mathrm{C}$ and $200^{\circ} \mathrm{C}$ points lie very close to the predicted mean. The $300^{\circ} \mathrm{C}$ point is potentially overaged (based on microstructural evidence of a partial cellular transformation ${ }^{8}$ ), though it nonetheless lies within the PI bounds.

The U-7.7Nb validation data (Figures 4.32-4.38) are in good agreement with predicted mean values for TE, 1YS, and 2YS, with marginal agreement for UE and 1YM. Poor agreement obtained for UTS, whose $200^{\circ} \mathrm{C}$ point lies outside of the PI bounds. For the HV, good agreement was obtained for $100^{\circ} \mathrm{C}$ and $200^{\circ} \mathrm{C}$, but the $300^{\circ} \mathrm{C}$ point fell outside of the $P I$ bounds. The $300^{\circ} \mathrm{C}$ point cannot be excluded on the grounds that overaging had begun, because no microstructural evidence for overaging (i.e., cellular decomposition products) was observed.

The banded U-6Nb validation data (Figures 4.39-4.45) were more difficult to compare with prediction because the extent of aging is much less after 5 years at $40^{\circ} \mathrm{C}, 65^{\circ} \mathrm{C}$, and (to a lesser extent) $90^{\circ} \mathrm{C}$ than it is after $\sim 0.6$ years at $100^{\circ} \mathrm{C}, 200^{\circ} \mathrm{C}$, and $300^{\circ} \mathrm{C}$. The TE and UE are difficult to compare with prediction because they are larger than the $P$ (start) value assigned to this alloy

\footnotetext{
${ }^{7}$ The PI bounds could also have been calculated on the assumption that they will be compared with individual replicate points (not their average), though this was not done in this study.

${ }^{8} \mathrm{HV}$ indent readings made in or near the cellular products were deliberately excluded from this data average; only those indents in the presumably unaffected matrix were considered. In this light, the $300^{\circ} \mathrm{C}-318,000$ minute $\mathrm{HV}$ point in U-5.6Nb can be considered physically legitimate if the typical assumption is made that a cellular transformation, one not governed by diffusion of an interstitial solute, does not affect the aging processes in the untransformed matrix removed a modest distance from the cellular-matrix growth front (say, >10 times the interlamellar spacing.)
} 
in Table 3.1. This is not surprising, because an extended time duration of initial softening might be expected to occur at these lower temperatures, longer in duration (given the lower temperature) than that seen at $100^{\circ} \mathrm{C}$ in U-5.6Nb (Figures 3.1 and 3.2) and U-7.7Nb. Until more validation data are available at $40^{\circ} \mathrm{C}, 65^{\circ} \mathrm{C}$, and $90^{\circ} \mathrm{C}$ for banded U-6Nb that better define a more accurate $P$ (start) value, comparison of TE and $\mathrm{UE}$ with prediction is not recommended. However, the negative result that little or no downward change occurred in the TE and UE after five years at these temperatures is not inconsistent with expectation; therefore, it is interpreted as a favorable sign. Among all the other properties (which did not show an initial softening at $100^{\circ} \mathrm{C}$ ), all lie within the PI bounds, with good-to-modest agreement with predicted mean values; the best agreement was obtained for 1YS and 2YS. The changes in UTS (both actual and predicted) were so small as to preclude meaningful comment, though at face value the agreement was quite good.

It is noted that the agreement between prediction and experiment for banded U-6Nb is good considering potential complications from (1) using the $f(x)$ model developed for nonbanded $\mathrm{U}-5.6 \mathrm{Nb}$ - a material of different chemical homogeneity and lower bulk $\mathrm{Nb}$ content, and (2) using a larger tensile geometry, which in addition (3) was tested in the AM condition. The tensile size and machining damage issues were expected to be partially factored out by assigning $P($ start) and $P($ peak) values specific for the banded U-6Nb in Table 3.1, and it appears as if this strategy was successful.

Overall, it is gratifying to observe that the vast majority of validation data agreed well with the model predictions, especially given the fact that these required extrapolations of the model, a more severe test of model fidelity and robustness than interpolations. This indicates that the validation specimens are largely from the same population as that from which the model was obtained, and the model does adequately represent the population. It gives added assurance that the property prediction models can be used with confidence. Upcoming validation points will provide a better test and give a better idea about just how much confidence can be placed in any given property model. The most important property, TE, was well-validated with U-7.7Nb, but the lack of any TE data for $\mathrm{U}-5.6 \mathrm{Nb}$ at the present time and expected interference from initial softening in banded U-6Nb necessitate a suspension of judgment on TE until further validation data become available.

Tables 4.7-4.13 lay out predicted values and associated PIs for all expected ages in both planned and already ongoing validation studies. At some point in the future, the available validation data can be used to update the model fits and predictions for future validation. In principle, this should be done as each validation point is gathered, though for practical reasons, this process will likely be done only when larger batches of new validation data are in hand.

\subsection{Lifetime Predictions}

Tables 4.14 and 4.15 provide the lifetime predictions for U-5.6Nb and U-7.7Nb, respectively. For ease of comparison with the lifetimes from the previous study (Table 3.4) and between alloys, an arbitrarily selected aging temperature, $40^{\circ} \mathrm{C}$ will be used; the relative comparisons are valid for all other temperatures. Compared with the previous study, the U-5.6Nb lower-bound lifetime decreased by a factor of $\sim 4$ from 7,759 to 1,828 years, whereas that of U-7.7Nb increased by a factor of $\sim 60$ from 95 to 5,466 years. The larger change in the U-7.7Nb relative to that of U-5.6Nb is certainly consistent with U-7.7Nb's greater nonlinearity in RSS (Appendix 3); therefore, its lower robustness of fit, relative to that of U-5.6Nb for TE. Even at aging 
temperatures as high as $60^{\circ} \mathrm{C}$, the minimum lifetimes from this present study are beyond 100 years, giving no cause for concern, if this failure criterion is accepted. It is reiterated that this failure criterion is considered overly simplistic; more detailed elaboration of this caveat can be found in the previous report [2007hac2].

\subsection{Evaluation of Age-Sensitive Properties for Surveillance}

A larger number of fitted properties was analyzed (relative to the previous study) to provide predictive models to support U-6Nb component surveillance studies and help address "what-if" questions that involve various aging (time-at-temperature) scenarios, for example, the impact of a transient thermal excursion above the normal storage temperature. The foregoing analysis (Section 4.1.2) indicated that 1YM and 1YS are the most age-sensitive properties from the standpoint of being the first to respond to age. From an engineering and surveillance point of view, the determination of the most age-sensitive property should also take into account the likely experimental errors, which show up in the form of scatter (e.g., standard deviation) of measurements carried out on replicate specimens. The practical threshold of ages that are most likely to give a statistically significant experimentally observable property change relative to the time $=0$ condition can be evaluated from knowledge of the scatter of the $P(t=0)$ point and the scatter of the short-time age points. This critical fraction transformed was defined as the point at which

$$
P(t)-P(t=0)>S D(t)+S D(t=0)
$$

Here the scatter is quantified in terms of the standard deviation $(S D)$ of the experimental measurements at time $=0$ and at low- $f$ ages. The SDs of the latter were defined as average $S D$ values for the $100^{\circ} \mathrm{C}$ aging data for $\mathrm{U}-5.6 \mathrm{Nb}$ and $\mathrm{U}-7.7 \mathrm{Nb}$, and the 5 -year ages at $40^{\circ} \mathrm{C}, 65^{\circ} \mathrm{C}$, and $90^{\circ} \mathrm{C}$ for banded U-6Nb. The threshold $P$ values resulting from Eq. 4.2, and their corresponding threshold $f$ values, appear in Tables 4.16-4.18. In all three alloys, 1YS and HV show consistently low thresholds, while TE and 1YM have consistently high thresholds. Thresholds for UE, 2YS, and UTS vary from alloy to alloy.

The aging time needed to exceed these thresholds can be evaluated from Eq. 3.25. This was done for a representative aging temperature of $40^{\circ} \mathrm{C}$, with results given in Tables 4.16, 4.17, and 4.18. The properties 1YS and 1YM show the earliest aging response (typically, on a timescale of weeks) in all three alloys; therefore, these are the most sensitive tripwires. The relatively high thresholds $(0.13 \leq f \leq 0.22)$ for 1 YM were more than offset by its low values of $Q$ (Table 4.4$)$ to give this early-time response (in comparison to those of other properties). It is also noted that 2YS and HV can show an early response, but only in U-7.7Nb.

The engineering interest of this threshold calculation lies in banded U-6Nb (Table 4.16), where changes in 1YS and 1YM could be observable in weeks or possibly days after the solutionizeand-quench processing step. This remarkably rapid response has the following caveat. The data are from one lot of RFP material, which may give a lower-than-normal $\Delta P$, at least for 1YS (8.1 MPa). Banded U-6Nb of Y-12 origin is known to have time $=0$, AM condition values of 1YS ranging over 100-200 MPa. An alternative calculation of the times it takes to progress from a representative time $=0$ value of 157.3 MPa to $200 \mathrm{MPa}$ gave times of 251 days, 3 years, and 196 years for the lower bound, mean, and upper bound aging times at $40^{\circ} \mathrm{C}$, respectively. (This row is denoted by the asterisk in Table 4.16) This shows that 1YS in Y-12 pedigree material is still expected to change on timescales of surveillance interest, though on a scale of years, not a scale of months as is the case for RFP pedigree material. Similar differences, 
between Y-12 and RFP pedigrees, in the threshold response of other properties may also occur, though at the present time there is scant data that would facilitate a quantitative evaluation like that done for 1YS.

The differences between the banded U-6Nb and the nonbanded U-5.6Nb and U-7.7Nb are quite pronounced when comparing the threshold values in Tables 4.16 with those of Tables 4.17 and 4.18. The threshold of $f$ in $\mathrm{U}-6 \mathrm{Nb}$ is comparable or lower than that of the nonbanded alloys in all instances. ${ }^{9}$ This leads to the conclusion that the much higher degree of redundant hot work experienced by banded U-6Nb and/or the larger tensile specimen size employed (both of which lower the scatter) far outweighs the expected increase in mechanical response scatter because of its higher chemical inhomogeneity vis-a-vis the nonbanded alloys that had less hot work and were tested with smaller tensile specimens.

In summary, the most sensitive tripwires to detect an aging process in U-Nb alloys-which are defined by a combination of early-time aging responses and relatively low experimental scatter-were determined to be 1YM, 1YS, and in some instances, HV. This finding is consistent with long-standing observations of rapid changes in 1YS with age relative to other properties, particularly TE and UTS, for example in U-4.6Nb and U-6.4Nb [1967jac], U-4.2Nb [1971jac], and U-4.5Nb [1976jac].

\subsection{Recommendations for Future Work}

Any future reexaminations of U-Nb aging with goals similar to those that motivated this study might benefit from improvements prompted mainly by the examination of the assumptions outlined in Section 4.1.3 and the acquisition of more data. To this end, the following recommendations are made:

1. An analysis identical to that taken in this study can be carried out with the benefit of a larger model-fitting data set. The validation data used in this study are obvious examples. Additionally, the potentially overaged data points excluded at the outset of this study can now be released for legitimate use in model fitting in view of microstructural evidence (Section 2.1) and theoretical considerations (see footnote in Section 4.3) that came to light during the course of this work.

2. The models can be improved by additional data that will reduce the residual errors and the $C I$ and $P I$ bounds, assuming the models are basically sound. Any additional data should concentrate on the domain of sub- $200^{\circ} \mathrm{C}$ aging temperatures. The top three recommended temperatures are $125^{\circ} \mathrm{C}, 150^{\circ} \mathrm{C}$, and $175^{\circ} \mathrm{C}$. Such data will help shed light on whether the aging mechanism truly changes (at least in a phenomenological sense), and if so, define more precisely the temperature that the mechanisms cross from one to another. Recall the instances of high uncertainty caused by "high-leverage" $100^{\circ} \mathrm{C}$ data points (Section 4.1.2) identified in both nonbanded alloys examined in the this study. ${ }^{10}$ This addresses assumption \#8.

\footnotetext{
${ }^{9}$ The one exception to this trend, HV, is likely due to the fact that the microhardness traces will capture the local spatial variations in $\mathrm{Nb}$ content in the microstructure (whereas these are averaged out in a tensile specimen), which are much larger in the banded U-6Nb than for the nonbanded U-5.6Nb and U-7.7Nb.

${ }^{10}$ High leverage arising from outlying $100^{\circ} \mathrm{C}$ data was also revealed in a similar analysis of microhardness data (shown in Figure 3.5 in [2007hac2]) obtained from banded Y-12 U-6Nb that was aged at similar times and temperatures as the nonbanded alloys in this study.
} 
3. The functional forms of model fitting at any given temperature could be reexamined, especially if additional data become available. Specific topics in this regard include, in order of increasing complexity:

a. Re-examine (and modify as needed) the assumption (\#3) that P(start) and P(peak) are independent of aging temperature. At a minimum, error bars can be placed on these parameters and the errors propagated through the model fitting process. Also update $\mathrm{P}$ (peak) for the 1YS, 2YS, and UTS - further consideration indicated that the $1,200 \mathrm{MPa}$ value for $\mathrm{U}-5.6 \mathrm{Nb}$ is probably too low, and would be set at 1,400 MPa or at an updated value for $\mathrm{U}-7.7 \mathrm{Nb}$ (say, 1,500 MPa), whichever is higher.

b. Further study of the phenomenology and time-temperature dependence of the apparent age-softening transient that precedes the age-hardening mechanism is desirable. A better understanding of this will result in an improved value of $P$ (start).

c. Reexamine, and modify as needed, the relationship of $P$ to $f$. In this study, it was assumed to be a simple linear transformation (Eq. 3.1). Other aging models, backed up by more physical insight, have proposed different functional forms [1994rob, 2002guo1, 2002guo2].

d. The time-series model-fitting equation could be reexamined (assumption \#6). After the probation of various functional forms (Section 3.3), the Logistic-cum-Boltzmann function (Eq. 3.18) was deemed the most appropriate to fit the data at hand, but additional data may prompt an exploration of other functional forms of the time-series equation.

e. A reexamination of the temperature-series function, the Arrhenius one (Eq. 3.6, assumption \#7), is not recommended in the absence of a physically plausible alternative - too few alternative models are available at the present time.

4. A systematic study should be undertaken of the effects of tensile geometry and machining damage state (AM vs MA) on the various properties on material of otherwise identical pedigree and heat treatment. One could envision that a property equivalency table would result from such a study, along the lines of that shown in Table 4.19. This would address (and provide quantitative correction for) the provisional assumption, made in this study, that the validation tensile data from banded U-6Nb could be directly compared with the model prediction data in spite of differences in both geometry and machining damage state. It would also give more confidence to the first recommendation of using existing validation data as model-fitting data. This addresses assumption \#9.

5. Develop an improved understanding of the physical aging mechanism(s), to address assumptions 1 and 2. Recent 3D atom probe results effectively ruled out Nb-diffusioncontrolled phase separation mechanisms at $200^{\circ} \mathrm{C}$ and presumably lower-still aging temperatures [2008cla]. The finding of this study that the 1YM is the earliest-changing property might suggest certain alternative avenues of investigation, for example, those involving internal friction or resonant ultrasound spectroscopy measurements.

6. The failure criterion should be critically reexamined with input from the relevant stakeholders. As mentioned at the outset, uncertainty in the nature and magnitude of the failure criterion for U-6Nb components is expected to be the most significant contributor to uncertainty in the lifetime evaluation. 


\section{CONCLUSIONS}

A more systematic and statistically aware kinetics modeling approach than employed previously gave reasonable model fits to accelerated aging property data in U-5.6Nb and U-7.7Nb, and useful age-sensitive property predictions for most of the properties studied, including TE, UE, 1YS, 2YS, and HV. This approach shared all of the fitting data, and many of the key assumptions of the previous approach, including the assumption of Arrhenius behavior. The key findings of this work include the following:

1. The activation energies for TE were $32 \mathrm{kcal} / \mathrm{mol}$ for $\mathrm{U}-5.6 \mathrm{Nb}$, in line with the previous study, but $39 \mathrm{kcal} / \mathrm{mol}$ for $\mathrm{U}-7.7 \mathrm{Nb}, 50 \%$ higher than the $Q$ value in the previous study. In light of recent atom probe studies, the closeness of these values to that for $\mathrm{Nb}$ diffusion in uranium, $32.6 \mathrm{kcal} / \mathrm{mol}$, may merely be coincidental.

2. The models generally showed more or less reasonable goodnesses of fit for both $\mathrm{U}-5.6 \mathrm{Nb}$ and U-7.7Nb, which vary from property to property. The best model fits were obtained for 2YS and HV, 1YM fit the least well, and the other properties' fits were in-between.

3. The U-5.6Nb models are more robust and symmetric, and therefore are expected to have better predictive power than those of U-7.7Nb, especially at the lower aging temperatures of interest.

4. Model extrapolations to longer times, and in some cases, lower temperatures than used for the model fitting agreed well with most of the validation data gathered, giving provisional validation of the fitted models.

5. The consistency of the banded $\mathrm{U}-6 \mathrm{Nb}$ validation data with the model predictions ratified the choice of using the model parameters fitted from nonbanded U-5.6Nb data, in the face of potential complexities owing to the materials' different bulk Nb contents, $\mathrm{Nb}$ homogeneity, tensile specimen size, and state of machining damage.

6. Even at aging temperatures as high as $60^{\circ} \mathrm{C}$, the minimum lifetimes from this present study are beyond 100 years, giving no cause for concern, if the failure criterion based on uniaxial tensile elongation is accepted, along with its serious caveats.

7. For surveillance purposes, $1 \mathrm{YM}, 1 \mathrm{YS}$, and to a lesser extent, $\mathrm{HV}$ are the properties most amenable to the early detection of the onset of aging.

8. The models can be improved by additional data that will test the various assumptions and, if the models are basically sound, reduce the residual errors and the $C I$ and $P I$ bounds. The additional data deemed of highest priority should concentrate on the domain of sub- $200^{\circ} \mathrm{C}$ aging temperatures. 


\section{ACKNOWLEDGEMENTS}

Robert Aikin, Jason Cooley, Paul Dunn, Duncan Hammon, Larry Hults, Dan Thoma, Phil Tubesing, and Chastity Vigil are thanked for their roles in planning and establishing the longterm aging experiments and associated apparatus within MST-6. David Alexander, John Balog, Tim Beard, Robert Forsyth, Denny Guidry, Ann Kelly, Martin Koby, Kathy Lao, Mike Lopez, Manny Lovato, Pallas Papin, Amanda Smith and Tim Tucker are thanked for assistance with specimen machining, heat treatment, testing, characterization, and analyses for the validation aging experiments. We also acknowledge useful discussions with Mike Hamada, Aparna Huzurbazar, Jennifer Lillard, Richard Picard, David Teter, and Alyson Wilson.

\section{REFERENCES}

1967jac R.J. Jackson, R.P. Brugger, and D.V. Miley, “Tensile Properties of Gamma Quenched and Aged Uranium-Rich Niobium Alloys,” Rocky Flats Plant, report RFP-933 (June 2, 1967).

1971jac R.J. Jackson and J.F. Boland, "Mechanical Properties of Uranium-Base Niobium Alloys,” Rocky Flats Plant, report RFP-1703 (June 17, 1971).

1974net J. Neter and W. Wasserman, Applied Linear Statistical Models, (Richard D. Irwin, Inc., Homewood, IL, 1974).

1976eck K.H. Eckelmeyer, “Aging Phenomena in Dilute Uranium Alloys,” in Physical Metallurgy of Uranium Alloys, J.J. Burke, D.A. Colling, A.E. Gorum, and J. Greenspan, ed. (Brook Hill, Chestnut Hill, MA, 1976), pp. 463-509.

1976jac R.J. Jackson, "Elastic, Plastic and Strength Properties of U-Nb and U-Nb-Zr Alloys,” in Physical Metallurgy of Uranium Alloys, J.J. Burke, D.A. Colling, A.E. Gorum, and J. Greenspan, ed. (Brook Hill, Chestnut Hill, MA, 1976), pp. 611-656.

1978kle D. Kleinbaum and L. Kupper, Applied Regression Analysis and Other Multivariable Methods. (Duxbury Press, North Scituate, MA, 1978).

1978sny1 W.B. Snyder, "Homogenization of Arc-Melted Uranium-6 Weight Percent Niobium Alloy Ingots,” Oak Ridge Y-12 Plant, report Y-2102 (January 31, 1978).

1978sny2 W.B. Snyder, "Fabrication and Characterization of Uranium-6 Niobium Alloy Plate with Improved Homogeneity,” Oak Ridge Y-12 Plant, report Y-2135 (October 31, 1978).

1981dra N.R. Draper and H. Smith, Applied Regression Analysis, 2nd ed. (Wiley, New York, 1981).

1982mee W.Q. Meeker and G.J. Hahn, "Sample sizes for prediction intervals,” Journal of Quality Technology 14, 201-206 (1982).

1983rat D.A. Ratkowsky, Nonlinear Regression Modeling. (Marcel Dekker, New York, 1983).

1984eck K.H. Eckelmeyer, A.D. Romig, Jr., and L.J. Weirick, “The Effect of Quench Rate on the Microstructure, Mechanical Properties, and Corrosion Behavior of U-6 Wt Pct Nb,” Metallurgical Transactions A 15A, 1319-1330 (1984). 
1988bat D.M. Bates and D.G. Watts, Nonlinear Regression Analysis and Its Applications. (Wiley, New York, 1988).

1990rat D.A. Ratkowsky, Handbook of Nonlinear Regression Models. (Marcel Dekker, New York, 1990).

1991hah G.J. Hahn and W.Q. Meeker, Statistical Intervals. (Wiley, New York, 1991).

1994rob ～C.V. Robino, M.J. Cieslak, P.W. Hochanadel, and G.R. Edwards, "Heat Treatment of Investment Cast PH 13-8 Mo Stainless Steel 2: Isothermal Aging Kinetics,” Metallurgical Transactions A 25A, 697-704 (1994).

1994ven W.N. Venables, and B.D. Ripley, Modern Applied Statistics with S-Plus. (Springer-Verlag, New York, 1994).

2002eck K.H. Eckelmeyer and D.J. Thoma, “Age Hardening of U-6wt\%Nb: Kinetic Analysis of Existing Heat Treatment Data and Prediction of Property Changes During Long Term Storage,” Los Alamos National Laboratory report LA-UR-02-7188 (June 2002).

2002 guo1 Z. Guo, W. Sha, and E.A. Wilson, "Modeling of Precipitation Kinetics and Age Hardening of Fe-12Ni-6Mn Maraging Type Alloy,” Materials Science and Technology 18, 377-382 (2002).

2002 guo2 Z. Guo and E.A. Wilson, "Quantification of Precipitation Kinetics and Age Hardening of Fe-12Ni-6Mn Alloy during Overaging,” Materials Science and Technology 18, 529-533 (2002).

2007hac1 R.E. Hackenberg, R.M. Aikin, Jr., J.A. Balog, B.L. Bingham, R. Casey, A. Casteel, I. Cordova, R. Forsyth, F.G. Garcia, D. Guidry, D.L. Hammon, W.L. Hults, D.R. Korzekwa, A.M. Kelly, M.W. Koby, K.A. Lao, J.C. Lashley, M.F. Lopez, R. McCabe, D.E. Nye, P.A. Papin, S.W. Quintana, J.L. Smith, D.F. Teter, D.J. Thoma, T. Tucker, P.K. Tubesing, R.R. Trujillo, C.J. Vigil, and H.M. Volz:, "Synthesis and Characterization of Nonbanded U-Nb Plate Material," Los Alamos National Laboratory report LA-14316 (January 2007).

2007hac2 R.E. Hackenberg, D.W. Brown, A.J. Clarke, L.B. Dauelsberg, R.D. Field, W.L. Hults, A.M. Kelly, M.F. Lopez, D.F. Teter, D.J. Thoma, T.J. Tucker, C.J. Vigil, and H.M. Volz, "U-Nb Aging Final Report," Los Alamos National Laboratory report LA-14327 (May 2007).

2008ale D.J. Alexander, private communication to REH, February 2008.

2008cla A.J. Clarke, R.D. Field, R.E. Hackenberg, D.J. Thoma, D.W. Brown, D.F. Teter, M.K. Miller, K.F. Russell, D.V. Edmonds, and G. Beverini, "Low Temperature Age-Hardening in U-13at.\%Nb: An Atom Probe Study,” J. Nuclear Materials, submitted (2008). Also available as LANL Report LA-UR-08-05743 (2008). 
Table 2.1. Tensile Data from Each Replicate of Nonbanded U-5.6Nb. Elongations are dimensionless; strengths and moduli are in MPa.

\begin{tabular}{|c|c|c|c|c|c|c|c|c|c|c|c|c|}
\hline \multirow{3}{*}{$\begin{array}{l}\text { Aging } \\
\text { Temp. } \\
(\mathrm{C})\end{array}$} & \multirow{3}{*}{$\begin{array}{l}\text { Aging } \\
\text { Time } \\
\text { (min.) }\end{array}$} & \multirow{3}{*}{$\begin{array}{l}\text { Rep. } \\
\#\end{array}$} & \multicolumn{9}{|c|}{ Engineering Stress-Strain Curve Parameters } & \multirow[t]{3}{*}{ Notes } \\
\hline & & & \multirow{2}{*}{\begin{tabular}{|l|} 
SYS \\
stress
\end{tabular}} & \multirow{2}{*}{$\begin{array}{l}1 Y S \\
\text { slope }\end{array}$} & \multirow{2}{*}{\begin{tabular}{|l|}
$2 Y S$ \\
stress
\end{tabular}} & \multirow{2}{*}{$\begin{array}{l}2 \text { YS } \\
\text { slope }\end{array}$} & \multirow{2}{*}{$\begin{array}{l}\text { UTS } \\
\text { stress }\end{array}$} & \multicolumn{3}{|c|}{ Plastic Elongation } & \multirow[t]{2}{*}{$\%$ RA } & \\
\hline & & & & & & & & Uniform & $\begin{array}{l}\text { Non- } \\
\text { Uniform }\end{array}$ & Total & & \\
\hline 23 & $<360$ & 1 & 107 & 37000 & 501 & 16673 & 715 & 0.2186 & 0.0269 & 0.2456 & 42.37 & $\mathrm{AQ}$ \\
\hline 23 & $<360$ & 2 & 148 & 70000 & 618 & 9803 & 873 & 0.1896 & 0.0063 & 0.1959 & 17.16 & $\overline{\mathrm{AQ}}$ \\
\hline 23 & $<360$ & $\overline{3}$ & 112 & 57000 & 507 & 13711 & 747 & 0.2039 & 0.0084 & 0.2122 & 22.13 & $\mathrm{AQ}$ \\
\hline 23 & $<360$ & 4 & 132 & 80000 & 553 & 10667 & 819 & 0.1969 & 0.0055 & 0.2025 & 24.12 & $\overrightarrow{A Q}$ \\
\hline 23 & $<360$ & 6 & 128 & 75000 & 547 & 10804 & 806 & 0.1938 & 0.0084 & 0.2022 & 34.33 & $\overline{\mathrm{AQ}}$ \\
\hline & & & & & & & & & & & & \\
\hline 100 & 10 & 1 & 124 & 60000 & 530 & 11446 & 745 & 0.1630 & 0.0063 & 0.1693 & 18.59 & \\
\hline 100 & 10 & 2 & 121 & 55000 & 519 & 12527 & 791 & 0.2149 & 0.0077 & 0.2227 & 24.83 & \\
\hline 100 & 100 & 1 & 114 & 62000 & 512 & 16489 & 742 & 0.2331 & 0.0343 & 0.2673 & 32.26 & \\
\hline 100 & 100 & 2 & 136 & 90000 & 536 & 11002 & 808 & 0.2100 & 0.0101 & 0.2202 & 33.64 & \\
\hline 100 & 165 & $\overline{1}$ & 139 & 52000 & 517 & 14321 & 771 & 0.2396 & 0.0629 & 0.3025 & 37.20 & \\
\hline 100 & 165 & 2 & 142 & 90000 & 544 & 11127 & 755 & 0.1555 & 0.0119 & 0.1674 & 17.05 & \\
\hline 100 & 215 & 1 & 178 & 55000 & 640 & 9949 & 902 & 0.2138 & 0.0040 & 0.2178 & 15.43 & \\
\hline 100 & 215 & 2 & 149 & 54000 & 534 & 10768 & 820 & 0.2491 & 0.0515 & 0.3006 & 28.22 & \\
\hline 100 & 1000 & 1 & 160 & 67000 & 531 & 11233 & 814 & 0.2478 & 0.0413 & 0.2890 & 32.05 & \\
\hline 100 & 1000 & $\overline{3}$ & 167 & 5000 & 508 & 16736 & 700 & 0.2247 & 0.0148 & 0.2395 & 24.09 & \\
\hline 100 & 10000 & 1 & 164 & 8000 & 563 & 15480 & 817 & 0.2338 & .0294 & 0.2632 & 31.47 & \\
\hline 100 & $\overline{000}$ & 2 & 169 & 70000 & 570 & 15395 & 821 & 0.2202 & 0.0158 & 0.2360 & 28.69 & \\
\hline 100 & $\overline{0000}$ & 1 & 225 & 66000 & 542 & 15051 & 757 & 0.2179 & 0.0108 & 0.2286 & 23.41 & \\
\hline 100 & 100000 & 2 & 223 & 86000 & 595 & 13409 & 845 & 0.2144 & 0.0069 & 0.2214 & 18.57 & \\
\hline & & & & & & & & & & & & \\
\hline 200 & 10 & 1 & 240 & 55000 & 595 & 12857 & 807 & 0.2049 & 0.0059 & 0.2108 & 21.88 & \\
\hline 200 & 10 & 3 & 208 & 50000 & 581 & 11809 & 811 & 0.2044 & 0.0046 & 0.2091 & 19.15 & \\
\hline 200 & 100 & $\overline{1}$ & 368 & 85000 & 640 & 8577 & 852 & 0.2228 & 0.0100 & 0.2328 & 22.47 & \\
\hline 200 & 100 & 2 & 202 & 38000 & 625 & 15516 & 758 & 0.2097 & 0.0257 & 0.2354 & 38.56 & \\
\hline 200 & 100 & 3 & 365 & 73000 & 641 & 7668 & 872 & 0.2498 & 0.0290 & 0.2788 & 27.85 & \\
\hline 200 & 1000 & 1 & 494 & 81000 & 788 & 7510 & 868 & 0.0797 & 0.0071 & 0.0868 & 8.55 & \\
\hline 200 & 1000 & 2 & 284 & 60000 & 720 & 11261 & 805 & 0.1881 & 0.0088 & 0.1969 & 19.89 & \\
\hline 200 & 1000 & 3 & 474 & 70000 & 737 & 5857 & 810 & 0.1324 & 0.0079 & 0.1403 & 13.86 & \\
\hline 200 & 10000 & 1 & 670 & 105000 & 880 & 2242 & 905 & 0.1092 & 0.0137 & 0.1229 & 10.23 & \\
\hline 200 & 10000 & 3 & 668 & 115000 & 857 & 3664 & 914 & 0.1125 & 0.0061 & 0.1186 & 13.16 & \\
\hline 200 & 100000 & 1 & 906 & 114000 & 1022 & 2847 & 1029 & 0.0670 & 0.0058 & 0.0728 & 17.69 & \\
\hline 200 & 100000 & 2 & 922 & 94000 & 978 & & 978 & 0.0021 & 0.0026 & 0.0046 & 9.07 & 2 \\
\hline 200 & 100000 & 3 & 762 & 75000 & 917 & 2756 & 924 & 0.0539 & 0.0055 & 0.0594 & 10.41 & \\
\hline 200 & 100000 & 4 & 960 & 95000 & 960 & & 1005 & 0.0045 & 0.0038 & 0.0083 & 12.73 & \\
\hline
\end{tabular}

Notes

AQ) Unaged "as-quenched" condition.

1) $2 Y S$ stress set equal to 1YS stress because they aren't distinguishable; $2 Y S$ slope indeterminate.

2) UTS stress set equal to $2 Y S$ stress because they aren't distinguishable; $2 Y S$ slope indeterminate.

3) Potentially overaged condition; not used in model fitting. 
Table 2.1 (continued). Tensile Data from Each Replicate of Nonbanded U-5.6Nb. Elongations are dimensionless; strengths and moduli are in MPa.

\begin{tabular}{|c|c|c|c|c|c|c|c|c|c|c|c|c|}
\hline \multirow{3}{*}{$\begin{array}{l}\text { Aging } \\
\text { Temp } \\
\text { (C) }\end{array}$} & \multirow{3}{*}{$\begin{array}{l}\text { Aging } \\
\text { Time } \\
\text { (min.) }\end{array}$} & \multirow{3}{*}{$\begin{array}{l}\text { Rep. } \\
\#\end{array}$} & \multicolumn{9}{|c|}{ Engineering Stress-Strain Curve Parameters } & \multirow[t]{3}{*}{ Notes } \\
\hline & & & \multirow{2}{*}{\begin{tabular}{|l|}
$1 Y S$ \\
stress \\
\end{tabular}} & \multirow{2}{*}{$\begin{array}{l}Y S \\
\text { slope }\end{array}$} & \multirow{2}{*}{$\begin{array}{l}2 Y S \\
\text { stress }\end{array}$} & \multirow{2}{*}{$\begin{array}{l}\text { YYS } \\
\text { slope }\end{array}$} & \multirow{2}{*}{\begin{tabular}{|l|} 
UTS \\
stress
\end{tabular}} & \multicolumn{3}{|c|}{ Plastic Elongation } & \multirow[t]{2}{*}{$\%$ RA } & \\
\hline & & & & & & & & Uniform & $\begin{array}{l}\text { Non- } \\
\text { Uniform }\end{array}$ & Total & & \\
\hline 250 & 10 & 1 & 387 & 110000 & 778 & 6916 & 920 & 0.1451 & 0.0039 & 0.1490 & 19.19 & \\
\hline 250 & 10 & $\overline{2}$ & 178 & 55000 & 630 & 12935 & 759 & 0.1307 & 0.0017 & 0.1324 & 17.06 & \\
\hline 250 & 100 & 1 & 227 & 62000 & 716 & 12186 & 875 & 0.2044 & 0.0483 & 0.2526 & 30.53 & \\
\hline 250 & 100 & 2 & 299 & 75000 & 749 & 10391 & 858 & 0.1233 & $\overline{041}$ & .1274 & 14.60 & \\
\hline 250 & 100 & $\overline{3}$ & 443 & 65000 & 801 & 4490 & 885 & 0.1256 & 0.0065 & 0.1322 & 12.46 & \\
\hline 250 & 1000 & 1 & 392 & 65000 & 842 & 9774 & 923 & 0.1342 & 0.0044 & 0.1386 & 19.88 & \\
\hline 250 & 1000 & 2 & 413 & 70000 & 845 & 9206 & 932 & 0.1275 & 0.0040 & 0.1315 & 11.98 & \\
\hline 250 & 10000 & $\overline{1}$ & 925 & 87000 & 998 & & 998 & 0.0047 & 0.0005 & 0.0052 & 3.86 & 2 \\
\hline 250 & 10000 & 2 & 1058 & 121000 & 1177 & & 1177 & 0.0071 & 0.0009 & 0.0080 & 5.12 & 2 \\
\hline 250 & 100000 & 1 & 1039 & 100000 & 1145 & & 1145 & 0.0048 & $\overline{08}$ & 0.0056 & 1.78 & 2,3 \\
\hline 250 & 100000 & 2 & 890 & 110000 & 890 & & 890 & 0.0000 & 0.0000 & 0.0000 & 0.82 & $\overline{1,2,3}$ \\
\hline & & & & & & & & & & & & \\
\hline 300 & 10 & 1 & 296 & 60000 & 837 & 10661 & 1000 & 0.1617 & 0.0067 & 0.1684 & 17.38 & \\
\hline 300 & 10 & 2 & 255 & 60000 & 806 & 12597 & 904 & 0.0653 & 0.0007 & 0.0660 & 11.43 & \\
\hline 300 & 100 & 1 & 539 & 74000 & 980 & 9834 & 1041 & 0.0596 & 0.0032 & 0.0627 & 5.64 & \\
\hline 300 & 100 & 2 & 494 & 84000 & 957 & 9005 & 1025 & 0.0718 & 0.0030 & 0.0748 & 9.47 & \\
\hline 300 & 1000 & 1 & 927 & 80000 & 1013 & & 1013 & 0.0027 & 0.0002 & 0.0029 & 0.14 & 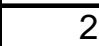 \\
\hline 300 & 1000 & $\overline{2}$ & $\overline{799}$ & 120000 & 1067 & & 1067 & 0.0064 & 0.0006 & 0.0070 & 2.27 & 5 \\
\hline 300 & 10000 & 1 & 1039 & 115000 & 1039 & & 1039 & 0.0000 & 0.0000 & 0.0000 & 2.98 & $\overline{1,2}$ \\
\hline 300 & 10000 & 2 & 1249 & 113000 & 1249 & & 1249 & 0.0000 & 0.0000 & 0.0000 & 2.51 & 1,2 \\
\hline 300 & 100000 & 1 & 966 & 124000 & 966 & & 966 & 0.0000 & 0.0000 & 0.0000 & 0.60 & $\overline{1,2,3}$ \\
\hline 300 & 100000 & 2 & 942 & 160000 & 942 & & 942 & 0.0000 & 0.0000 & 0.0000 & 2.17 & $1,2,3$ \\
\hline
\end{tabular}

Notes

AQ) Unaged "as-quenched" condition.

1) $2 Y S$ stress set equal to 1YS stress because they aren't distinguishable; $2 Y S$ slope indeterminate.

2) UTS stress set equal to $2 Y S$ stress because they aren't distinguishable; $2 Y S$ slope indeterminate.

3) Potentially overaged condition; not used in model fitting. 
Table 2.2. Vickers Microhardness Linescan Data from Nonbanded U-5.6Nb.

\begin{tabular}{|c|c|c|c|c|c|c|c|c|c|}
\hline Agin & p. (C) & 23 & 100 & 100 & 100 & 100 & 100 & 100 & 100 \\
\hline Agin & (min.) & $<360$ & 10 & 100 & 165 & 215 & 1000 & 10000 & 100000 \\
\hline Note & & AQ & & & & & & & \\
\hline Inde & $\operatorname{orce}(\mathrm{g})$ & 500 & 500 & 500 & 500 & 500 & 500 & 500 & 500 \\
\hline Spa & nicron) & 250 & 210 & 210 & 210 & 210 & 210 & 210 & 210 \\
\hline $\mathrm{HV}$ & Average & 147.2 & 147.1 & 153.1 & 156.0 & 160.7 & 163.1 & 163.4 & 159.1 \\
\hline & SD & 7.9 & 13.0 & 9.8 & 6.3 & 6.3 & 8.2 & 8.0 & 6.1 \\
\hline & Individual & 164 & 173 & 168 & 166 & 168 & 173 & 165 & 163 \\
\hline & Data & 144 & 166 & 160 & 148 & 172 & 164 & 168 & 162 \\
\hline & Points & 155 & 152 & 153 & 159 & 165 & 167 & 149 & 154 \\
\hline & & 146 & 147 & 144 & 154 & 165 & 176 & 150 & 167 \\
\hline & & 143 & 145 & 143 & 145 & 158 & 158 & 162 & 167 \\
\hline & & 147 & 145 & 135 & 160 & 157 & 157 & 169 & 158 \\
\hline & & 146 & 139 & 157 & 163 & 161 & 158 & 162 & 150 \\
\hline & & 151 & 147 & 146 & 154 & 155 & 158 & 156 & 155 \\
\hline & & 139 & 143 & 161 & 151 & 152 & 165 & 164 & 158 \\
\hline & & 137 & 127 & 156 & 153 & 155 & 146 & 175 & 150 \\
\hline & & & 151 & 164 & 158 & 155 & 165 & 159 & 159 \\
\hline & & & 130 & 150 & 161 & 165 & 170 & 176 & 166 \\
\hline & & & & & & & & 164 & \\
\hline & & & & & & & & 168 & \\
\hline
\end{tabular}

\begin{tabular}{|c|c|c|c|c|c|c|}
\hline \multicolumn{2}{|c|}{ Aging temp. (C) } & 200 & 200 & 200 & 200 & 200 \\
\hline \multicolumn{2}{|c|}{ Aging time (min.) } & 10 & 100 & 1000 & 10000 & 100000 \\
\hline \multicolumn{7}{|c|}{\begin{tabular}{|l|} 
Notes \\
\end{tabular}} \\
\hline \multicolumn{2}{|c|}{\begin{tabular}{|l|} 
Indenter force $(\mathrm{g})$ \\
\end{tabular}} & 500 & 500 & 50 & 500 & 500 \\
\hline \multicolumn{2}{|c|}{ Spacing (micron) } & 200 & 210 & 200 & 170 & 210 \\
\hline \multirow[t]{17}{*}{$\mathrm{HV}$} & Average & 177.8 & 194.5 & 246.2 & 284.6 & 331.9 \\
\hline & SD & 8.2 & 6.0 & 9.3 & 7.1 & 6.9 \\
\hline & \multirow{15}{*}{\begin{tabular}{|l} 
Individual \\
Data \\
Points
\end{tabular}} & 171 & 210 & 248 & 288 & 331 \\
\hline & & 176 & 194 & 240 & 281 & 334 \\
\hline & & 163 & 199 & 265 & 282 & 330 \\
\hline & & 176 & 189 & 243 & 274 & 337 \\
\hline & & 172 & 190 & 243 & 282 & 337 \\
\hline & & 178 & 192 & 233 & 280 & 317 \\
\hline & & 173 & 194 & 243 & 274 & 327 \\
\hline & & 183 & 187 & 235 & 282 & 322 \\
\hline & & 188 & 192 & 238 & 284 & 338 \\
\hline & & 180 & 195 & 248 & 285 & 338 \\
\hline & & 194 & 194 & 256 & 282 & 333 \\
\hline & & 180 & 198 & 256 & 291 & 339 \\
\hline & & & & 253 & 301 & \\
\hline & & & & & 289 & \\
\hline & & & & & 294 & \\
\hline
\end{tabular}

\section{Notes}

AQ) Unaged "as-quenched" condition.

1) Potentially overaged condition. 
Table 2.2 (continued). Vickers Microhardness Linescan Data from Nonbanded U-5.6Nb.

\begin{tabular}{|c|c|c|c|c|c|c|}
\hline \multicolumn{2}{|c|}{ Aging temp. (C) } & 250 & 250 & 250 & 250 & 250 \\
\hline \multicolumn{2}{|c|}{ Aging time (min.) } & 10 & 100 & 1000 & 10000 & 100000 \\
\hline \multicolumn{2}{|c|}{ Notes } & & & & & 1 \\
\hline \multicolumn{2}{|c|}{ Indenter force (g) } & 500 & 50 & 500 & 500 & 500 \\
\hline \multicolumn{2}{|c|}{ Spacing (micron) } & 210 & 200 & 210 & 175 & 210 \\
\hline \multirow[t]{16}{*}{$\mathrm{HV}$} & Average & 189.4 & 252.2 & 265.6 & 316.8 & 407.8 \\
\hline & SD & 7.6 & 21.1 & 14.7 & 8.0 & 9.2 \\
\hline & \multirow{14}{*}{$\begin{array}{l}\text { Individual } \\
\text { Data } \\
\text { Points }\end{array}$} & 191 & 265 & 274 & 320 & 407 \\
\hline & & 188 & 268 & 277 & 315 & 409 \\
\hline & & 195 & 308 & 247 & 324 & 416 \\
\hline & & 180 & 245 & 250 & 317 & 422 \\
\hline & & 182 & 265 & 249 & 327 & 416 \\
\hline & & 185 & 243 & 261 & 298 & 411 \\
\hline & & 182 & 253 & 261 & 306 & 406 \\
\hline & & 182 & 245 & 260 & 315 & 399 \\
\hline & & 192 & 223 & 258 & 320 & 399 \\
\hline & & 193 & 240 & 272 & 317 & 413 \\
\hline & & 204 & 248 & 284 & 322 & 407 \\
\hline & & 199 & 235 & 294 & 321 & 388 \\
\hline & & & 240 & & 308 & \\
\hline & & & & & 325 & \\
\hline
\end{tabular}

\begin{tabular}{|c|c|c|c|c|c|c|}
\hline \multicolumn{2}{|c|}{ Aging temp. (C) } & 300 & 300 & 300 & 300 & 300 \\
\hline \multicolumn{2}{|c|}{ Aging time (min.) } & 10 & 100 & 1000 & 10000 & 100000 \\
\hline \multicolumn{2}{|c|}{ Notes } & & & & & 1 \\
\hline \multicolumn{2}{|c|}{ Indenter force $(\mathrm{g})$} & 500 & 500 & 500 & 500 & 500 \\
\hline \multicolumn{2}{|c|}{ Spacing (micron) } & 175 & 175 & 175 & 175 & 210 \\
\hline \multirow[t]{16}{*}{$\mathrm{HV}$} & Average & 217.4 & 273.9 & 357.1 & 427.4 & 468.10 \\
\hline & SD & 15.8 & 8.1 & 11.0 & 13.4 & 14.40 \\
\hline & \multirow{14}{*}{$\begin{array}{l}\text { Individual } \\
\text { Data } \\
\text { Points }\end{array}$} & 195 & 277 & 356 & 433 & 480 \\
\hline & & 228 & 274 & 362 & 439 & 458 \\
\hline & & 210 & 279 & 360 & 434 & 491 \\
\hline & & 202 & 265 & 349 & 426 & 468 \\
\hline & & 208 & 261 & 366 & 407 & 480 \\
\hline & & 211 & 264 & 362 & 435 & 475 \\
\hline & & 199 & 264 & 337 & 415 & 458 \\
\hline & & 214 & 277 & 349 & 445 & 458 \\
\hline & & 214 & 271 & 341 & 431 & 445 \\
\hline & & 223 & 271 & 369 & 413 & 449 \\
\hline & & 234 & 279 & 369 & 429 & 473 \\
\hline & & 239 & 284 & 346 & 445 & 482 \\
\hline & & 217 & 281 & 371 & 430 & \\
\hline & & 250 & 287 & 363 & 402 & \\
\hline
\end{tabular}

Notes

AQ) Unaged "as-quenched" condition.

1) Potentially overaged condition. 
Table 2.3. Tensile Data from Each Replicate of Nonbanded U-7.7Nb. Elongations are dimensionless; strengths and moduli are in MPa.

\begin{tabular}{|c|c|c|c|c|c|c|c|c|c|c|c|c|}
\hline \multirow{3}{*}{$\begin{array}{l}\text { Aging } \\
\text { Temp. } \\
\text { (C) }\end{array}$} & \multirow{3}{*}{$\begin{array}{l}\text { Aging } \\
\text { Time } \\
\text { (min.) }\end{array}$} & \multirow{3}{*}{$\begin{array}{l}\text { Rep. } \\
\#\end{array}$} & \multicolumn{9}{|c|}{ Engineering Stress-Strain Curve Parameters } & \multirow[t]{3}{*}{ Notes } \\
\hline & & & \multirow{2}{*}{\begin{tabular}{|l|}
$1 Y S$ \\
stress
\end{tabular}} & \multirow{2}{*}{$\begin{array}{l}\text { YS } \\
\text { slope }\end{array}$} & \multirow{2}{*}{$\begin{array}{l}2 Y S \\
\text { stress }\end{array}$} & \multirow{2}{*}{$\begin{array}{l}\text { YS } \\
\text { slope }\end{array}$} & \multirow{2}{*}{\begin{tabular}{|l|} 
UTS \\
stress
\end{tabular}} & \multicolumn{3}{|c|}{ Plastic Elongation } & \multirow[t]{2}{*}{$\%$ RA } & \\
\hline & & & & & & & & Uniform & \begin{tabular}{|l|} 
Non- \\
Uniform
\end{tabular} & Total & & \\
\hline 23 & $<360$ & 1 & 110 & 27000 & 546 & 16800 & 729 & 0.2885 & 0.0507 & 0.3391 & 53.94 & $\mathrm{AQ}$ \\
\hline 23 & $<360$ & 2 & 100 & 32000 & 507 & 19013 & 719 & 0.2862 & 0.1952 & 0.4814 & 49.71 & $\overrightarrow{\mathrm{AQ}}$ \\
\hline 23 & $<360$ & 3 & 95 & 25000 & 522 & 17658 & 686 & 0.1985 & 0.0084 & 0.2069 & 25.98 & $\mathrm{AQ}$ \\
\hline 23 & $<360$ & $\overline{4}$ & 94 & 32000 & 500 & 19334 & 694 & 0.2349 & 0.0096 & 0.2445 & 26.25 & $\overrightarrow{A Q}$ \\
\hline 23 & $<360$ & 5 & 96 & 26000 & 496 & 16601 & 684 & 0.2829 & 0.1508 & 0.4337 & 50.15 & $\mathrm{AQ}$ \\
\hline 23 & $<360$ & 6 & 84 & 52000 & 495 & 16771 & 672 & 0.2733 & 0.0316 & 0.3050 & 30.29 & $\overrightarrow{\mathrm{AQ}}$ \\
\hline 23 & $<360$ & $\overline{7}$ & 98 & 40000 & 499 & 17559 & 709 & 0.2683 & 0.1186 & 0.3869 & 58.16 & $\overrightarrow{A Q}$ \\
\hline & & & & & & & & & & & & \\
\hline 100 & 10 & $\overline{1}$ & $\overline{113}$ & 43000 & 556 & 19256 & 720 & 0.2847 & 0.0725 & 0.3572 & 49.99 & \\
\hline 100 & 10 & 2 & 136 & 28000 & 590 & 17722 & 726 & 0.2833 & 0.1389 & 0.4222 & 57.82 & \\
\hline 100 & 100 & 1 & 215 & 33000 & 606 & 17071 & 733 & 0.2770 & 0.1068 & 0.3837 & 58.37 & \\
\hline 100 & 100 & 2 & 188 & 29000 & 586 & 16891 & 726 & 0.2644 & 0.0412 & 0.3055 & 29.82 & \\
\hline 100 & 1000 & 1 & 300 & 43000 & 632 & 14833 & 739 & 0.2652 & 0.0735 & 0.3387 & 63.18 & \\
\hline 100 & 1000 & 2 & 330 & 48000 & 648 & 14940 & 747 & 0.2312 & 0.0442 & 0.2754 & 50.57 & \\
\hline 100 & 10000 & 1 & 546 & 42000 & 617 & 9660 & 744 & 0.2163 & 0.1319 & 0.3482 & 56.78 & \\
\hline 100 & 10000 & 2 & 476 & 46000 & 638 & 3158 & 725 & 0.2349 & 0.0920 & 0.3269 & 58.85 & \\
\hline 100 & 230385 & 1 & 589 & 53000 & 762 & 4638 & 784 & 0.2290 & 0.0412 & 0.2703 & 51.68 & \\
\hline 100 & 230385 & 2 & 534 & 55000 & 710 & 6449 & 785 & 0.2726 & 0.1095 & 0.3822 & 43.85 & \\
\hline & & & & & & & & & & & & \\
\hline 200 & 10 & $\overline{1}$ & 269 & 31000 & 656 & 16067 & 742 & 0.2118 & 0.0777 & 0.2895 & 56.02 & \\
\hline 200 & 10 & 2 & 155 & 31000 & 630 & 18299 & 733 & 0.2459 & 0.0481 & 0.2940 & 57.91 & \\
\hline 200 & 100 & 1 & 153 & 37000 & 630 & 16409 & 759 & 0.2638 & 0.0216 & 0.2854 & 26.45 & \\
\hline 200 & 100 & 2 & 318 & 29000 & 702 & 14943 & 760 & 0.2184 & 0.0363 & 0.2548 & 27.77 & \\
\hline 200 & 1000 & 1 & 296 & 55000 & 730 & 13419 & 811 & 0.2346 & 0.0345 & 0.2691 & 42.41 & \\
\hline 200 & 1000 & 2 & 284 & 45000 & 770 & 11621 & 796 & 0.2058 & 0.0789 & 0.2847 & 44.92 & \\
\hline 200 & 10000 & 1 & 611 & 52000 & 855 & 6540 & 874 & 0.1347 & 0.0648 & 0.1994 & 25.80 & \\
\hline 200 & 10000 & 2 & 576 & 56000 & 868 & 6466 & 880 & 0.0549 & 0.0460 & 0.1009 & 39.46 & \\
\hline 200 & 10000 & 3 & 404 & 30000 & 768 & 5220 & 793 & 0.1096 & 0.0259 & 0.1355 & 14.50 & \\
\hline 200 & 100000 & 1 & 763 & 65000 & 979 & 2482 & 987 & 0.0501 & 0.1061 & 0.1562 & 27.08 & \\
\hline 200 & 100000 & 2 & 806 & 60000 & 982 & 1597 & 986 & 0.0471 & 0.0466 & 0.0937 & 24.78 & \\
\hline
\end{tabular}

Notes

AQ) Unaged "as-quenched" condition.

1) $2 Y S$ stress set equal to 1YS stress because they aren't distinguishable; $2 Y S$ slope indeterminate.

2) UTS stress set equal to $2 Y S$ stress because they aren't distinguishable; $2 Y S$ slope indeterminate.

3) Potentially overaged condition; not used in model fitting. 
Table 2.3 (continued). Tensile Data from Each Replicate of Nonbanded U-7.7Nb. Elongations are dimensionless; strengths and moduli are in MPa.

\begin{tabular}{|c|c|c|c|c|c|c|c|c|c|c|c|c|}
\hline \multirow{3}{*}{$\begin{array}{l}\text { Aging } \\
\text { Temp. } \\
\text { (C) }\end{array}$} & \multirow{3}{*}{$\begin{array}{l}\text { Aging } \\
\text { Time } \\
\text { (min.) }\end{array}$} & \multirow{3}{*}{$\begin{array}{l}\text { Rep. } \\
\#\end{array}$} & \multicolumn{9}{|c|}{ Engineering Stress-Strain Curve Parameters } & \multirow[t]{3}{*}{ Notes } \\
\hline & & & \multirow{2}{*}{\begin{tabular}{|l|}
$1 Y S$ \\
stress
\end{tabular}} & \multirow{2}{*}{\begin{tabular}{|l|}
$1 Y S$ \\
slope
\end{tabular}} & \multirow{2}{*}{\begin{tabular}{|l|}
$2 Y S$ \\
stress
\end{tabular}} & \multirow{2}{*}{$\begin{array}{l}2 Y S \\
\text { slope }\end{array}$} & \multirow{2}{*}{$\begin{array}{l}\text { UTS } \\
\text { stress }\end{array}$} & \multicolumn{3}{|c|}{ Plastic Elongation } & \multirow[t]{2}{*}{$\%$ RA } & \\
\hline & & & & & & & & Uniform & \begin{tabular}{|l|} 
Non- \\
Uniform
\end{tabular} & Total & & \\
\hline 250 & 10 & 1 & 146 & 45000 & 746 & 19173 & 794 & 0.2065 & 0.0323 & 0.2388 & 47.94 & \\
\hline 250 & 10 & 2 & 170 & 38000 & 774 & 20382 & 809 & 0.1274 & 0.0605 & 0.1880 & 50.31 & \\
\hline 250 & 100 & 1 & 187 & 41000 & 861 & 20791 & 894 & 0.0277 & 0.0370 & 0.0647 & 42.66 & \\
\hline 250 & 100 & 2 & 191 & 40000 & 855 & 21047 & 870 & 0.0264 & 0.0064 & 0.0328 & 46.97 & \\
\hline 250 & 1000 & 1 & 412 & 51000 & 1018 & 14571 & 1046 & 0.0305 & 0.0164 & 0.0469 & 30.31 & \\
\hline 250 & 1000 & 2 & 522 & 50000 & 1017 & 12998 & 1047 & 0.0335 & 0.0106 & 0.0441 & 31.18 & \\
\hline 250 & 10000 & 1 & 988 & 73000 & 988 & & 1058 & 0.0058 & 0.0008 & 0.0066 & 0.89 & 1 \\
\hline 250 & 10000 & 2 & 961 & 73000 & 1166 & 2780 & 1169 & 0.0427 & 0.0096 & 0.0523 & 17.99 & \\
\hline 250 & 10000 & 3 & 728 & 78000 & 1105 & 5697 & 1116 & 0.0416 & 0.0207 & 0.0623 & 21.86 & \\
\hline 250 & 10000 & 4 & 775 & 72000 & 1118 & 5580 & 1123 & 0.0374 & 0.0094 & 0.0468 & 21.89 & \\
\hline 250 & 100000 & 1 & 1216 & 75000 & 1216 & & 1225 & 0.0022 & 0.0015 & 0.0037 & 11.19 & $\perp$ \\
\hline 250 & 100000 & 2 & 1176 & 98000 & 1176 & & 1229 & 0.0066 & 0.0023 & 0.0089 & 9.58 & 1 \\
\hline & & & & & & & & & & & & \\
\hline 300 & 10 & 1 & 232 & 37000 & 856 & 21375 & 900 & 0.1189 & 0.1541 & 0.2731 & 38.21 & \\
\hline 300 & 10 & 2 & 242 & 40000 & 871 & 21171 & 908 & 0.0309 & 0.1239 & 0.1548 & 36.97 & \\
\hline 300 & 100 & 1 & 378 & 38000 & 1041 & 19672 & 1096 & 0.0338 & 0.0329 & 0.0667 & 23.28 & \\
\hline 300 & 100 & 2 & 394 & 34000 & 1045 & 17869 & 1095 & 0.0352 & 0.0216 & 0.0568 & 19.22 & \\
\hline 300 & 1000 & 1 & 1177 & 68000 & 1177 & & 1261 & 0.0131 & 0.0000 & 0.0131 & 0.36 & $\perp$ \\
\hline 300 & 1000 & 2 & 1124 & 63000 & 1124 & & 1234 & 0.0063 & 0.0027 & 0.0091 & 1.54 & \\
\hline 300 & 10000 & 1 & 1340 & 77000 & 1340 & & 1340 & 0.0000 & 0.0000 & 0.0000 & 1.10 & $1,2,3$ \\
\hline 300 & 10000 & 2 & 1268 & 76000 & 1268 & & 1268 & 0.0000 & 0.0000 & 0.0000 & 0.92 & $1,2,3$ \\
\hline 300 & 100000 & 1 & 1137 & 76000 & 1137 & & 1137 & 0.0000 & 0.0000 & 0.0000 & 1.33 & $1,2,3$ \\
\hline 300 & 100000 & 2 & 1213 & 81000 & 1213 & & 1213 & 0.0000 & 0.0000 & 0.0000 & 2.68 & $1,2,3$ \\
\hline
\end{tabular}

Notes

AQ) Unaged "as-quenched" condition.

1) $2 Y S$ stress set equal to 1YS stress because they aren't distinguishable; $2 Y S$ slope indeterminate.

2) UTS stress set equal to $2 Y S$ stress because they aren't distinguishable; $2 Y S$ slope indeterminate.

3) Potentially overaged condition; not used in model fitting. 
Table 2.4. Vickers Microhardness Linescan Data from Nonbanded U-7.7Nb.

\begin{tabular}{|c|c|c|c|c|c|c|c|}
\hline Agin & o. (C) & 23 & 100 & 100 & 100 & 100 & 100 \\
\hline Agin & $(\min )$. & $<360$ & 10 & 100 & 1000 & 10000 & 230385 \\
\hline Note & & $\mathrm{AQ}$ & & & & & \\
\hline Inde & rce $(\mathrm{g})$ & 500 & 500 & 500 & 500 & 500 & 500 \\
\hline Spa & nicron) & 194 & 234 & 235 & 233 & 236 & 250 \\
\hline $\mathrm{HV}$ & Average & 115.2 & 145.6 & 158.5 & 157.2 & 192.6 & 214.8 \\
\hline & SD & 2.2 & 5.2 & 6.4 & 7.6 & 4.9 & 3.5 \\
\hline & | Individual & 116 & 139 & 171 & 139 & 185 & 214 \\
\hline & Data & 120 & 135 & 164 & 156 & 196 & 221 \\
\hline & Points & 112 & 145 & 156 & 153 & 198 & 210 \\
\hline & & 113 & 146 & 149 & 165 & 190 & 212 \\
\hline & & 115 & 149 & 161 & 162 & 191 & 217 \\
\hline & & 114 & 145 & 159 & 159 & 198 & 214 \\
\hline & & 115 & 149 & 151 & 167 & 192 & 215 \\
\hline & & 117 & 143 & 155 & 158 & 185 & 215 \\
\hline & & 115 & 147 & 154 & 161 & 196 & 219 \\
\hline & & 115 & 151 & 161 & 157 & 195 & 211 \\
\hline & & & 153 & 163 & 152 & & \\
\hline
\end{tabular}

\begin{tabular}{|c|c|c|c|c|c|c|}
\hline \multicolumn{2}{|c|}{ Aging temp. (C) } & 200 & 200 & 200 & 200 & 200 \\
\hline \multicolumn{2}{|c|}{ Aging time (min.) } & 10 & 100 & 1000 & 10000 & 100000 \\
\hline \multicolumn{7}{|c|}{ Notes } \\
\hline \multicolumn{2}{|c|}{ Indenter force $(\mathrm{g})$} & 500 & 500 & 500 & 500 & 500 \\
\hline \multicolumn{2}{|c|}{ Spacing (micron) } & 315 & 293 & 335 & 335 & 261 \\
\hline \multirow[t]{11}{*}{$\mathrm{HV}$} & Average & 147.9 & 179.0 & 204.8 & 229.4 & 272.6 \\
\hline & SD & 6.7 & 7.1 & 10.8 & 8.8 & 7.3 \\
\hline & \multirow{9}{*}{\begin{tabular}{|l} 
Individual \\
Data \\
Points
\end{tabular}} & 155 & 172 & 195 & 215 & 275 \\
\hline & & 149 & 189 & 194 & 227 & 275 \\
\hline & & 151 & 175 & 195 & 229 & 273 \\
\hline & & 145 & 176 & 199 & 239 & 269 \\
\hline & & 151 & 183 & 204 & 243 & 282 \\
\hline & & 148 & 184 & 220 & 227 & 273 \\
\hline & & 151 & 188 & 218 & 232 & 262 \\
\hline & & 133 & 170 & 213 & 223 & 282 \\
\hline & & & 174 & & & 262 \\
\hline
\end{tabular}

Notes

AQ) Unaged "as-quenched" condition.

1) Potentially overaged condition. 
Table 2.4 (continued). Vickers Microhardness Linescan Data from Nonbanded U-7.7Nb.

\begin{tabular}{|c|c|c|c|c|c|c|}
\hline \multicolumn{2}{|c|}{ Aging temp. (C) } & 250 & 250 & 250 & 250 & 250 \\
\hline \multicolumn{2}{|c|}{ Aging time (min.) } & 10 & 100 & 1000 & 10000 & 100000 \\
\hline \multicolumn{7}{|c|}{ Notes } \\
\hline \multicolumn{2}{|c|}{ Indenter force $(\mathrm{g})$} & 500 & 500 & 500 & 500 & 500 \\
\hline \multicolumn{2}{|c|}{ Spacing (micron) } & 373 & 350 & 350 & 350 & 275 \\
\hline \multirow[t]{10}{*}{$\mathrm{HV}$} & Average & 172.7 & 183.3 & 230.6 & 321.4 & 372.6 \\
\hline & SD & 2.6 & 4.6 & 4.0 & 8.2 & 11.7 \\
\hline & \multirow{8}{*}{\begin{tabular}{|l|} 
Individual \\
Data \\
Points
\end{tabular}} & 175 & 175 & 225 & $\overline{316}$ & 356 \\
\hline & & 172 & 189 & 230 & 315 & 380 \\
\hline & & 175 & 184 & 225 & 334 & 369 \\
\hline & & 171 & 182 & 234 & 324 & 380 \\
\hline & & 173 & 187 & 233 & 325 & 392 \\
\hline & & 175 & 181 & 233 & 310 & 374 \\
\hline & & 168 & 185 & 234 & 326 & 359 \\
\hline & & & & & & 371 \\
\hline
\end{tabular}

\begin{tabular}{|c|c|c|c|c|c|c|}
\hline Agin & p. (C) & 300 & 300 & 300 & 300 & 300 \\
\hline Agin & (min.) & 10 & 100 & 1000 & 10000 & 100000 \\
\hline Note & & & & & 1 & 1 \\
\hline Inde & $\operatorname{prce}(\mathrm{g})$ & 500 & 500 & 500 & 500 & 500 \\
\hline Spa & nicron) & 325 & 293 & 329 & 275 & 217 \\
\hline $\mathrm{HV}$ & Average & 181.5 & 234.4 & 365.9 & 416.0 & 488.8 \\
\hline & SD & 6.5 & 5.8 & 10.4 & 8.7 & 8.0 \\
\hline & \begin{tabular}{|l|} 
Individua \\
\end{tabular} & 192 & 233 & 353 & 409 & 487 \\
\hline & Data & 181 & 247 & 374 & 404 & 482 \\
\hline & Points & 183 & 228 & 355 & 426 & 489 \\
\hline & & 180 & 238 & 380 & 422 & 491 \\
\hline & & 185 & 228 & 374 & 415 & 477 \\
\hline & & 185 & 236 & 365 & 433 & 496 \\
\hline & & 171 & 232 & 371 & 416 & 484 \\
\hline & & 175 & 236 & 355 & 413 & 489 \\
\hline & & & 232 & & 409 & 501 \\
\hline & & & & & 413 & 501 \\
\hline & & & & & & 480 \\
\hline
\end{tabular}

Notes

AQ) Unaged "as-quenched" condition.

1) Potentially overaged condition. 
Table 2.5. Tensile Validation Data for Nonbanded U-7.7Nb. Elongations are dimensionless; strengths and moduli are in MPa.

\begin{tabular}{|c|c|c|c|c|c|c|c|c|c|c|c|}
\hline \multirow{3}{*}{$\begin{array}{l}\text { Aging } \\
\text { Temp } \\
\text { (C) }\end{array}$} & \multirow{3}{*}{$\begin{array}{l}\text { Aging } \\
\text { Time } \\
\text { (min.) }\end{array}$} & \multirow{3}{*}{$\begin{array}{l}\text { Rep. } \\
\#\end{array}$} & \multicolumn{9}{|c|}{ Engineering Stress-Strain Curve Parameters } \\
\hline & & & \multirow{2}{*}{\begin{tabular}{|l|}
$1 Y S$ \\
stress
\end{tabular}} & \multirow{2}{*}{$\begin{array}{l}1 Y S \\
\text { slope }\end{array}$} & \multirow{2}{*}{\begin{tabular}{|l|}
$2 Y S$ \\
stress
\end{tabular}} & \multirow{2}{*}{$\begin{array}{l}\mathrm{YS} \\
\text { slope }\end{array}$} & \multirow{2}{*}{\begin{tabular}{|l|} 
UTS \\
stress
\end{tabular}} & \multicolumn{3}{|c|}{ Plastic Elongation } & \multirow[t]{2}{*}{$\%$ RA } \\
\hline & & & & & & & & Uniform & Total & \begin{tabular}{|l|} 
Non- \\
Uniform
\end{tabular} & \\
\hline 100 & & 1 & 546 & 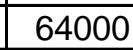 & 703 & 46 & 754 & & & & 48. \\
\hline 100 & & 2 & 451 & 65000 & 655 & 8822 & 763 & 0.2352 & 096 & 44 & 56.78 \\
\hline 200 & 318000 & 1 & 978 & 84000 & 1084 & 1034 & 1084 & 0.0450 & 0.0732 & 0.0282 & 18.37 \\
\hline 200 & 318000 & 2 & 997 & 79000 & 1119 & 1466 & 1121 & 0.0440 & 0.0833 & 0.0394 & 15.36 \\
\hline
\end{tabular}


Table 2.6. Vickers Hardness Validation Data for Nonbanded U-5.6Nb. The individual HV data point lists are split into two columns, running top-to-bottom, then left-to-right. Blank spaces indicate the locations of points that gave invalid $\mathrm{HV}$ readings because of inclusion or edge effects.

\begin{tabular}{|c|c|c|c|c|c|c|c|}
\hline \multicolumn{2}{|c|}{ Aging temp. (C) } & \multicolumn{2}{|l|}{100} & \multicolumn{2}{|l|}{200} & \multicolumn{2}{|l|}{300} \\
\hline \multicolumn{2}{|c|}{ Aging time (min.) } & \multicolumn{2}{|l|}{318000} & \multicolumn{2}{|l|}{318000} & \multicolumn{2}{|l|}{318000} \\
\hline \multicolumn{2}{|c|}{ Replicate } & \multicolumn{2}{|c|}{1} & \multicolumn{2}{|l|}{1} & \multicolumn{2}{|l|}{1} \\
\hline \multicolumn{2}{|c|}{ Notes } & & & & & \multicolumn{2}{|l|}{1} \\
\hline \multirow{2}{*}{\multicolumn{2}{|c|}{\begin{tabular}{|l} 
Indenter force $(\mathrm{g})$ \\
Snacing (micron)
\end{tabular}}} & \multicolumn{2}{|l|}{50} & \multicolumn{2}{|l|}{50} & 50 & \\
\hline & nicron) & \multicolumn{2}{|c|}{50} & \multicolumn{2}{|l|}{50} & \multicolumn{2}{|l|}{50} \\
\hline \multirow[t]{30}{*}{$\mathrm{HV}$} & Average & 188.7 & & 366.5 & & 529.6 & \\
\hline & SD & \multicolumn{2}{|l|}{7.9} & \multicolumn{2}{|l|}{12.8} & \multicolumn{2}{|l|}{24.0} \\
\hline & \multirow{28}{*}{$\begin{array}{l}\text { Individual } \\
\text { Data } \\
\text { Points }\end{array}$} & & 182 & 373 & 378 & 564 & 546 \\
\hline & & 214 & 192 & 363 & 359 & 529 & 520 \\
\hline & & 200 & 196 & 383 & 363 & 537 & 546 \\
\hline & & 198 & 192 & 363 & 368 & 529 & 520 \\
\hline & & 192 & 190 & 349 & 373 & 537 & 504 \\
\hline & & 187 & 182 & 388 & 328 & 504 & 489 \\
\hline & & 200 & 189 & 388 & 363 & 546 & 504 \\
\hline & & 192 & 202 & 378 & 349 & 555 & 512 \\
\hline & & 194 & 200 & 378 & 363 & 520 & 504 \\
\hline & & 198 & 198 & 363 & 349 & 564 & 529 \\
\hline & & 192 & 189 & 373 & 359 & 529 & 537 \\
\hline & & 189 & 175 & 368 & 368 & 537 & 529 \\
\hline & & 187 & 183 & 373 & 373 & & 546 \\
\hline & & 182 & 182 & 368 & 368 & 546 & 537 \\
\hline & & 202 & 185 & 368 & 363 & 546 & 497 \\
\hline & & 185 & 183 & 368 & 363 & 546 & 529 \\
\hline & & 192 & 187 & 388 & 354 & 555 & 555 \\
\hline & & & 177 & 378 & & 546 & 564 \\
\hline & & 189 & 178 & & 373 & 546 & 555 \\
\hline & & 185 & 177 & 388 & 349 & 546 & \\
\hline & & 180 & 185 & 378 & 378 & 546 & \\
\hline & & & 177 & 373 & & 546 & 497 \\
\hline & & 187 & 183 & 368 & 363 & 482 & 482 \\
\hline & & 187 & 196 & 354 & 368 & 537 & 467 \\
\hline & & 187 & 190 & 373 & 345 & 546 & 497 \\
\hline & & 187 & 183 & 378 & 341 & 529 & 482 \\
\hline & & 183 & 177 & 373 & 345 & 546 & 529 \\
\hline & & 194 & & 359 & & 546 & \\
\hline
\end{tabular}

Notes

1) Potentially overaged condition. 
Table 2.7. Vickers Hardness Validation Data for Nonbanded U-7.7Nb. The individual HV data point lists are split into two columns, running top-to-bottom, then left-to-right. Blank spaces indicate the locations of points that gave invalid $\mathrm{HV}$ readings because of inclusion or edge effects. For the two ages having more than one replicate $\left(200^{\circ} \mathrm{C}\right.$ and $\left.300^{\circ} \mathrm{C}\right)$, only replicate \#2 data were used for the validation; replicate \#1 is listed for information only.

\begin{tabular}{|c|c|c|c|c|c|c|c|c|c|c|c|}
\hline \multicolumn{2}{|c|}{ Aging temp. (C) } & \multicolumn{2}{|l|}{100} & \multicolumn{2}{|l|}{200} & \multicolumn{2}{|l|}{200} & \multicolumn{2}{|l|}{300} & \multicolumn{2}{|l|}{300} \\
\hline & (min.) & 318000 & & 318000 & & 318000 & & 318000 & & 318000 & \\
\hline \multicolumn{2}{|c|}{ Replicate } & \multicolumn{2}{|l|}{\begin{tabular}{|r}
1 \\
\end{tabular}} & \multicolumn{2}{|l|}{$\begin{array}{r}318000 \\
1\end{array}$} & \multicolumn{2}{|l|}{2} & \multicolumn{2}{|l|}{1} & \multicolumn{2}{|l|}{2} \\
\hline \multicolumn{2}{|c|}{ Notes } & \multicolumn{2}{|l|}{2} & \multicolumn{2}{|l|}{2} & & \multicolumn{2}{|l|}{1,2} & \multicolumn{2}{|l|}{1} \\
\hline \multicolumn{2}{|c|}{ Indenter force (g) } & \multicolumn{2}{|l|}{50} & \multicolumn{2}{|l|}{50} & \multicolumn{2}{|l|}{50} & \multicolumn{2}{|l|}{50} & \multicolumn{2}{|l|}{50} \\
\hline \multicolumn{2}{|c|}{ Spacing (micron) } & 50 & & 50 & & 50 & & 50 & & 50 & \\
\hline $\mathrm{HV}$ & Average & 190.9 & & 306.8 & & 320.9 & & 518.5 & & 526.6 & \\
\hline & SD & 5.8 & & 13.9 & & 20.4 & & 26.3 & & 24.7 & \\
\hline & \begin{tabular}{|l|} 
Individual \\
\end{tabular} & 198 & 198 & 328 & 301 & 349 & 316 & 520 & 489 & 564 & 537 \\
\hline & Data & 189 & 185 & 308 & 290 & 312 & 324 & 537 & 482 & 573 & 520 \\
\hline & Points & 198 & 190 & 312 & 308 & 336 & 332 & 529 & 537 & 537 & 497 \\
\hline & & 196 & 202 & 324 & 294 & 332 & 328 & 529 & 512 & 546 & 497 \\
\hline & & 189 & 190 & 316 & 332 & 345 & 308 & 537 & 489 & 537 & 489 \\
\hline & & 187 & 190 & 320 & 336 & 332 & 320 & 564 & 489 & 546 & 489 \\
\hline & & 189 & 200 & 308 & & 328 & 308 & 537 & 520 & 564 & 489 \\
\hline & & 190 & 190 & 308 & 328 & 336 & 297 & 546 & 504 & 564 & 497 \\
\hline & & 189 & 196 & 320 & 320 & 316 & 297 & 520 & 475 & 573 & 504 \\
\hline & & 189 & 194 & 308 & 320 & 363 & 301 & 434 & 504 & 573 & 489 \\
\hline & & 178 & 192 & 312 & 316 & 359 & 290 & 537 & 497 & 555 & 504 \\
\hline & & 185 & 189 & 312 & 297 & 359 & 294 & 564 & 489 & & 520 \\
\hline & & 185 & 192 & 287 & 312 & 354 & 328 & 497 & 489 & 546 & 512 \\
\hline & & 190 & 189 & 297 & 308 & 345 & 305 & 537 & 512 & 546 & 489 \\
\hline & & 187 & 198 & 320 & & & 301 & 555 & 546 & 520 & 546 \\
\hline & & 182 & 192 & 297 & 284 & 312 & 297 & 520 & 537 & 537 & 504 \\
\hline & & 182 & 206 & & 301 & 312 & 297 & 537 & 520 & 537 & 489 \\
\hline & & 177 & 196 & 287 & 287 & 336 & 312 & 537 & 512 & 529 & 504 \\
\hline & & 180 & 192 & 290 & 305 & 308 & 336 & 529 & 537 & 520 & 537 \\
\hline & & 192 & 194 & 312 & 290 & 312 & 308 & 537 & 529 & 512 & 555 \\
\hline & & 187 & 198 & 332 & 280 & 332 & 280 & 497 & 537 & 512 & 520 \\
\hline & & 190 & 196 & 297 & 305 & 328 & 294 & 489 & 555 & & 520 \\
\hline & & 189 & 190 & 305 & 294 & 305 & 316 & 512 & 555 & 504 & 520 \\
\hline & & 194 & & 297 & & 316 & 312 & & & 512 & 529 \\
\hline & & 196 & & 320 & & 316 & 324 & & & 520 & 564 \\
\hline & & 192 & & 308 & & 336 & 294 & 497 & & 546 & 529 \\
\hline & & 189 & & 297 & & 363 & & 497 & & 529 & 537 \\
\hline & & 189 & & 294 & & 345 & & 497 & & 520 & \\
\hline
\end{tabular}

Notes

1) Potentially overaged condition.

2) This replicate specimen may be less chemically homogeneous than typical specimens of this alloy. 
Table 2.8. Chemical Analysis Results of Banded U-6Nb from the Validation Experiments. The units are in wppm; the SD is from multiple replicate measurements. The elements $\mathrm{H}$, $\mathrm{C}, \mathrm{N}, \mathrm{O}$, and $\mathrm{S}$ were analyzed as described in [2007hac1]. The remaining elements were analyzed by inductively coupled plasma-optical emission spectroscopy (regular font) or by inductively coupled plasma-mass spectroscopy (bold font), with the aliquot preparation technique as described in [2007hac1], with the exception that aqua regia was used for initial specimen dissolution. Less than $(<)$ indicates the method detection limit.

\begin{tabular}{|c|c|c|c|c|c|c|c|c|}
\hline & \multicolumn{2}{|c|}{ Unaged } & \multicolumn{2}{|c|}{ 40C-5 years } & \multicolumn{2}{|c|}{ 65C-5 years } & \multicolumn{2}{|c|}{ 90C-5 years } \\
\hline Element & Average & SD & Average & $\mathrm{SD}$ & Average & SD & Average & SD \\
\hline $\mathrm{H}$ & 0.05 & 0.08 & 0.04 & 0.03 & 0.09 & 0.09 & 0.17 & 0.05 \\
\hline $\mathrm{B}$ & $<25.50$ & & $<25.50$ & & $<25.50$ & & $<25.50$ & \\
\hline$C$ & 60.50 & 15.66 & 58.71 & 9.41 & 51.12 & 8.34 & 55.66 & 10.70 \\
\hline $\bar{N}$ & \begin{tabular}{l|l|}
5.02 \\
\end{tabular} & 1.15 & 4.68 & 1.06 & 7.93 & 1.15 & 5.15 & 1.07 \\
\hline 0 & 55.57 & 6.89 & 60.07 & 5.34 & 59.83 & 7.04 & 53.58 & 6.18 \\
\hline $\mathrm{Na}$ & $<19.10$ & & $<19.10$ & & $<19.10$ & & $<19.10$ & \\
\hline $\mathrm{Mg}$ & $<3.20$ & & $<3.20$ & & $<3.20$ & & $<3.20$ & \\
\hline Al & $<22.30$ & & $<22.30$ & & $<22.30$ & & $<22.30$ & \\
\hline Si & $<79.70$ & & $<79.70$ & & $<79.70$ & & $<79.70$ & \\
\hline $\mathrm{P}$ & $<510.00$ & & $<510.00$ & & $<510.00$ & & $<510.00$ & \\
\hline$S$ & $<4.10$ & & $<4.10$ & & $<4.10$ & & $<4.10$ & \\
\hline $\mathrm{K}$ & $<25.50$ & & $<25.50$ & & $<25.50$ & & $<25.50$ & \\
\hline $\mathrm{Ca}$ & $<31.90$ & & $<31.90$ & & $<31.90$ & & $<31.90$ & \\
\hline $\mathrm{Ti}$ & 3.84 & 0.83 & 3.23 & 0.25 & 2.90 & 0.10 & 1.90 & 1.39 \\
\hline V & $<22.30$ & & $<22.30$ & & $<22.30$ & & $<22.30$ & \\
\hline $\mathrm{Cr}$ & $<19.10$ & & $<19.10$ & & $<19.10$ & & $<19.10$ & \\
\hline $\mathrm{Mn}$ & 5.89 & 0.97 & 4.83 & 0.25 & 4.40 & 0.00 & 4.50 & 0.10 \\
\hline $\mathrm{Fe}$ & $<22.30$ & & $<22.30$ & & $<22.30$ & & $<22.30$ & \\
\hline Co & $<19.10$ & & $<19.10$ & & $<19.10$ & & $<19.10$ & \\
\hline $\mathrm{Ni}$ & 5.41 & 1.49 & 3.73 & 0.38 & 3.07 & 0.25 & 2.97 & 0.06 \\
\hline $\mathrm{Cu}$ & 7.05 & 1.92 & & & & & & \\
\hline $\mathrm{Zn}$ & 4.25 & 4.60 & & & & & & \\
\hline $\mathrm{Ga}$ & $<37.30$ & & $<37.30$ & & $<37.30$ & & $<37.30$ & \\
\hline $\mathrm{Ge}$ & $<210.00$ & & $<210.00$ & & $<210.00$ & & $<210.00$ & \\
\hline As & $<234.00$ & & $<234.00$ & & $<234.00$ & & $<234.00$ & \\
\hline \begin{tabular}{|l}
$\mathrm{Y}$ \\
\end{tabular} & $<3.20$ & & $<3.20$ & & $<3.20$ & & $<3.20$ & \\
\hline $\mathrm{Zr}$ & $<0.23$ & & 6.80 & 0.30 & 6.33 & 0.15 & 5.97 & 0.15 \\
\hline $\mathrm{Nb}$ & 62628.57 & 969.04 & 63300.00 & 793.73 & 64000.00 & 1609.35 & 63600.00 & 1178.98 \\
\hline Mo & $<35.10$ & & $<35.10$ & & $<35.10$ & & $<35.10$ & \\
\hline $\mathrm{Ru}$ & $<0.07$ & & $<0.07$ & & $<0.07$ & & $<0.07$ & \\
\hline $\mathrm{Cd}$ & $<9.60$ & & $<9.60$ & & $<9.60$ & & $<9.60$ & \\
\hline $\ln$ & $<166.00$ & & $<166.00$ & & $<166.00$ & & $<166.00$ & \\
\hline $\mathrm{Sb}$ & $<134.00$ & & $<134.00$ & & $<134.00$ & & $<134.00$ & \\
\hline $\mathrm{Ba}$ & 2.26 & 0.98 & 2.37 & 0.40 & 2.20 & 0.10 & 1.50 & 0.95 \\
\hline $\mathrm{Hf}$ & $<0.05$ & & $<0.05$ & & $<0.05$ & & $<0.05$ & \\
\hline $\mathrm{Ta}$ & 43.27 & 1.99 & 45.13 & 1.06 & 45.70 & 0.95 & 44.93 & 0.95 \\
\hline \begin{tabular}{|l}
$W$ \\
\end{tabular} & 2.84 & 0.08 & 2.83 & 0.12 & 3.13 & 0.12 & 3.03 & 0.12 \\
\hline $\operatorname{Re}$ & $<54.20$ & & $<54.20$ & & $<54.20$ & & $<54.20$ & \\
\hline $\mathrm{Pb}$ & $<89.30$ & & $<89.30$ & & $<89.30$ & & $<89.30$ & \\
\hline <3.20: Li, & e, Sc, Sr & & $<0.04:$ Cs, & & & & ICP-OES n & rmal \\
\hline$<0.12: \mathrm{Sr}$ & & & $<0.02: \mathrm{Rh}$, & Ag, La, & Tm, Tl, Th & & ICP-MS bo & \\
\hline
\end{tabular}


Table 2.9. Tensile Validation Data for Banded U-6Nb.

\begin{tabular}{|c|c|c|c|c|c|c|c|c|c|c|c|c|}
\hline \multirow{3}{*}{\begin{tabular}{|l} 
Aging \\
Temp. \\
(C)
\end{tabular}} & \multirow{3}{*}{$\begin{array}{l}\text { Aging } \\
\text { Time } \\
\text { (min.) }\end{array}$} & \multirow{3}{*}{$\begin{array}{l}\text { Rep. } \\
\#\end{array}$} & \multicolumn{9}{|c|}{ Engineering Stress-Strain Curve Parameters } & \multirow[t]{3}{*}{ Notes } \\
\hline & & & \multirow{2}{*}{\begin{tabular}{|l|}
$1 Y S$ \\
stress
\end{tabular}} & \multirow{2}{*}{$\begin{array}{l}1 Y S \\
\text { slope }\end{array}$} & \multirow{2}{*}{$\begin{array}{l}\mathrm{YS} \\
\text { stress }\end{array}$} & \multirow{2}{*}{$\begin{array}{l}2 \text { YS } \\
\text { slope }\end{array}$} & \multirow{2}{*}{$\begin{array}{l}\text { UTS } \\
\text { stress }\end{array}$} & \multicolumn{3}{|c|}{ Plastic Elongation } & \multirow[t]{2}{*}{$\%$ RA } & \\
\hline & & & & & & & & Uniform & Total & $\begin{array}{l}\text { Non- } \\
\text { Uniform }\end{array}$ & & \\
\hline 23 & $<135000$ & 1 & 15 & 60000 & 657 & 20602 & 821 & 0.2354 & 0.3930 & 0.1576 & 38.49 & \\
\hline 23 & $<135000$ & 2 & 16 & 50000 & 658 & 21047 & 819 & 0.2337 & 0.3490 & 0.1153 & 34.92 & \\
\hline 23 & $<135000$ & 3 & 15 & 50000 & 650 & 20315 & 816 & 0.2453 & 0.4339 & 0.1886 & 43.91 & \\
\hline 40 & 2629440 & 1 & 19 & 66000 & 674 & 19484 & 826 & 0.2476 & 0.3792 & 0.1316 & 38.93 & \\
\hline 40 & 2629440 & 2 & 19 & 70000 & 664 & 20580 & 823 & 0.2542 & 0.4110 & 0.1568 & 40.31 & \\
\hline 40 & 2629440 & 3 & 19 & 69000 & 663 & 20119 & 821 & 0.2458 & 0.3568 & 0.1110 & 39.63 & \\
\hline 65 & 2629440 & 1 & $26 !$ & 69000 & 680 & 17500 & 829 & 0.2508 & 0.4001 & 0.1494 & 37.92 & \\
\hline 65 & 2629440 & 2 & 268 & 72000 & 678 & 17607 & 835 & 0.2500 & 0.3606 & 0.1106 & 35.23 & \\
\hline 65 & 2629440 & 3 & 26 & 72000 & 673 & 17361 & 824 & 0.2598 & 0.3808 & 0.1209 & 32.82 & \\
\hline 90 & 2629440 & $\overline{1}$ & 39 & 82000 & 726 & 13129 & 832 & 0.2556 & 0.4140 & 0.1583 & 34.30 & \\
\hline 90 & 2629440 & 2 & 39 & 88000 & 722 & 13954 & 827 & 0.2550 & 0.4142 & 0.1592 & 39.81 & \\
\hline 90 & 2629440 & 3 & 39 & 79000 & 716 & 14187 & 828 & 0.2528 & 0.3879 & 0.1352 & 37.88 & \\
\hline
\end{tabular}


Table 2.10. Vickers Hardness Validation Data for Banded U-6Nb. The individual HV data point lists are split into five columns, running top-to-bottom, then left-to-right. Blank spaces indicate the locations of points that gave invalid $\mathrm{HV}$ readings because of inclusion or edge effects.

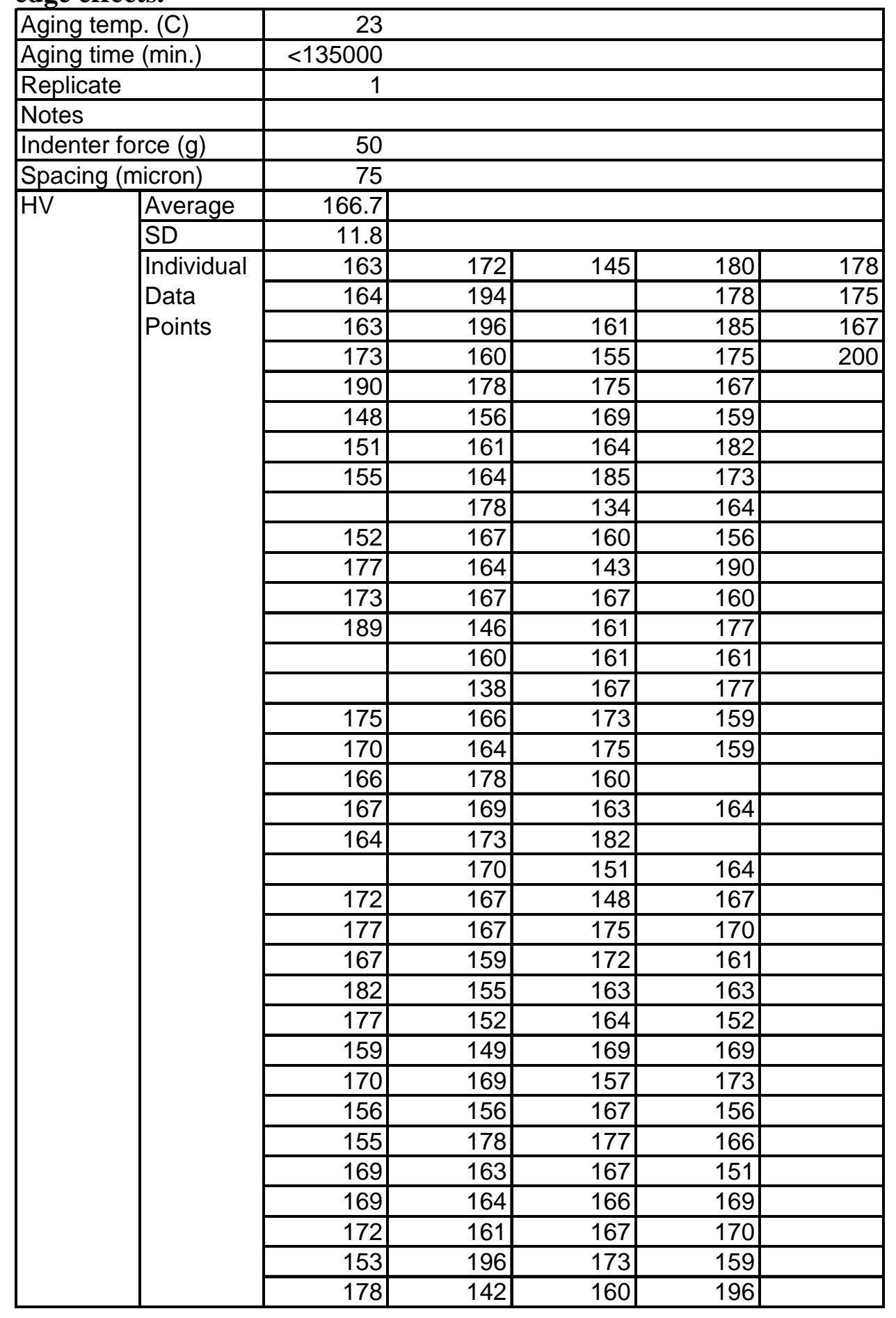


Table 2.10 (continued). Vickers Hardness Validation Data for Banded U-6Nb. The individual HV data point lists are split into five columns, running top-to-bottom, then leftto-right. Blank spaces indicate the locations of points that gave invalid $\mathrm{HV}$ readings because of inclusion or edge effects.

\begin{tabular}{|c|c|c|c|c|c|c|}
\hline \multicolumn{2}{|c|}{ Aging temp. (C) } & \multicolumn{5}{|l|}{40} \\
\hline \multicolumn{2}{|c|}{ Aging time (min.) } & \multicolumn{5}{|l|}{2629440} \\
\hline \multicolumn{2}{|c|}{ Replicate } & \multicolumn{3}{|l|}{1} & & \\
\hline \multicolumn{7}{|c|}{ Notes } \\
\hline \multicolumn{2}{|c|}{ Indenter force $(\mathrm{g})$} & \multicolumn{3}{|l|}{50} & & \\
\hline \multicolumn{2}{|c|}{ Spacing (micron) } & \multicolumn{3}{|l|}{65} & & \\
\hline \multirow[t]{37}{*}{$\mathrm{HV}$} & Average & \multicolumn{2}{|l|}{170.7} & & & \\
\hline & SD & 11.9 & & & & \\
\hline & \multirow{35}{*}{$\begin{array}{l}\text { Individual } \\
\text { Data } \\
\text { Points }\end{array}$} & 180 & 152 & 152 & 163 & 178 \\
\hline & & 169 & 153 & 172 & 177 & 178 \\
\hline & & 187 & 167 & 169 & 172 & 170 \\
\hline & & 161 & 160 & 192 & 172 & 161 \\
\hline & & 160 & 177 & 185 & 170 & 170 \\
\hline & & 160 & 185 & 196 & 169 & 178 \\
\hline & & 163 & 164 & 147 & 167 & 157 \\
\hline & & 169 & 163 & 148 & 177 & 190 \\
\hline & & 167 & 155 & 160 & 160 & 157 \\
\hline & & 170 & 170 & 159 & 166 & 157 \\
\hline & & 185 & 160 & 175 & 177 & 155 \\
\hline & & & 160 & 160 & 172 & 164 \\
\hline & & 172 & 172 & 161 & 178 & 175 \\
\hline & & 210 & 163 & 169 & 170 & 173 \\
\hline & & 178 & 178 & 164 & 146 & \\
\hline & & 164 & 173 & 169 & 177 & 175 \\
\hline & & 170 & 155 & 194 & 175 & 163 \\
\hline & & 173 & 149 & 175 & 180 & 175 \\
\hline & & 161 & 161 & 160 & 178 & 166 \\
\hline & & 194 & & 167 & 172 & 170 \\
\hline & & 159 & 177 & 170 & 166 & 170 \\
\hline & & 182 & 164 & 185 & 160 & 153 \\
\hline & & 163 & 166 & 167 & 170 & 196 \\
\hline & & 144 & 172 & 190 & 169 & 182 \\
\hline & & 175 & 167 & 183 & 175 & 183 \\
\hline & & 163 & & 190 & 173 & 182 \\
\hline & & 166 & 173 & 163 & 192 & \\
\hline & & 173 & 156 & 160 & 159 & \\
\hline & & 178 & 173 & 187 & 180 & \\
\hline & & & 180 & 166 & 167 & \\
\hline & & 177 & 190 & 172 & 182 & \\
\hline & & 152 & 182 & 159 & 177 & \\
\hline & & 190 & 177 & 152 & 182 & \\
\hline & & 167 & 182 & 160 & 214 & \\
\hline & & 170 & 163 & 166 & 189 & \\
\hline
\end{tabular}


Table 2.10 (continued). Vickers Hardness Validation Data for Banded U-6Nb. The individual HV data point lists are split into five columns, running top-to-bottom, then leftto-right. Blank spaces indicate the locations of points that gave invalid $\mathrm{HV}$ readings because of inclusion or edge effects.

\begin{tabular}{|c|c|c|c|c|c|c|}
\hline \multicolumn{2}{|c|}{ Aging temp. (C) } & \multicolumn{5}{|l|}{65} \\
\hline \multicolumn{2}{|c|}{ Aging time (min.) } & \multicolumn{5}{|l|}{2629440} \\
\hline \multicolumn{2}{|c|}{ Replicate } & \multicolumn{3}{|l|}{1} & & \\
\hline \multicolumn{7}{|c|}{ Notes } \\
\hline \multicolumn{2}{|c|}{ Indenter force $(\mathrm{g})$} & \multicolumn{3}{|l|}{50} & & \\
\hline \multicolumn{2}{|c|}{ Spacing (micron) } & \multicolumn{3}{|l|}{65} & & \\
\hline \multirow[t]{37}{*}{$\mathrm{HV}$} & Average & 181.5 & & & & \\
\hline & SD & 13.2 & & & & \\
\hline & \multirow{35}{*}{$\begin{array}{l}\text { Individual } \\
\text { Data } \\
\text { Points }\end{array}$} & 172 & 163 & 194 & 198 & 169 \\
\hline & & 192 & 190 & 185 & 177 & 169 \\
\hline & & 172 & 178 & 182 & 188 & 187 \\
\hline & & & 167 & 190 & 175 & 164 \\
\hline & & 187 & 187 & 172 & 183 & 160 \\
\hline & & 185 & 177 & 204 & 170 & 159 \\
\hline & & 169 & 183 & 210 & 190 & 167 \\
\hline & & 185 & 185 & 185 & 182 & 189 \\
\hline & & 167 & 182 & 183 & 196 & 155 \\
\hline & & 166 & 175 & 196 & 182 & \\
\hline & & 177 & 194 & 169 & 169 & 194 \\
\hline & & 170 & 187 & 183 & 190 & 192 \\
\hline & & 170 & 177 & 185 & 212 & 192 \\
\hline & & 167 & 185 & 204 & 182 & 177 \\
\hline & & 161 & & 208 & 190 & 172 \\
\hline & & 173 & 178 & 177 & 166 & 164 \\
\hline & & 163 & 202 & 163 & 166 & 169 \\
\hline & & 172 & 167 & 190 & 173 & 177 \\
\hline & & 187 & 163 & 182 & 204 & 173 \\
\hline & & 178 & 180 & 192 & 187 & 200 \\
\hline & & & 178 & 178 & 182 & 175 \\
\hline & & 177 & 183 & 200 & 190 & 157 \\
\hline & & 183 & 169 & 192 & 187 & 172 \\
\hline & & 175 & 187 & & 225 & 172 \\
\hline & & 194 & 189 & 182 & 237 & 216 \\
\hline & & 192 & 182 & 178 & 216 & 177 \\
\hline & & 189 & 175 & 189 & 178 & 196 \\
\hline & & 172 & 180 & 175 & 183 & 187 \\
\hline & & 187 & 183 & 180 & 173 & 167 \\
\hline & & 183 & 185 & 172 & & \\
\hline & & 208 & 177 & 183 & 173 & \\
\hline & & 200 & 177 & 178 & 169 & \\
\hline & & 185 & 182 & 177 & 172 & \\
\hline & & 164 & 189 & 180 & 166 & \\
\hline & & 157 & 189 & 182 & 178 & \\
\hline
\end{tabular}


Table 2.10 (continued). Vickers Hardness Validation Data for Banded U-6Nb. The individual HV data point lists are split into five columns, running top-to-bottom, then left-to-right. Blank spaces indicate the locations of points that gave invalid $\mathrm{HV}$ readings because of inclusion or edge effects.

\begin{tabular}{|c|c|c|c|c|c|c|}
\hline \multicolumn{2}{|c|}{ Aging temp. (C) } & 90 & & & & \\
\hline \multicolumn{2}{|c|}{ Aging time (min.) } & 2629440 & & & & \\
\hline \multicolumn{2}{|c|}{ Replicate } & 1 & & & & \\
\hline \multicolumn{7}{|c|}{ Notes } \\
\hline \multicolumn{2}{|c|}{ Indenter force $(\mathrm{g})$} & 50 & & & & \\
\hline \multicolumn{2}{|c|}{ Spacing (micron) } & 65 & & & & \\
\hline \multirow[t]{37}{*}{$\mathrm{HV}$} & Average & 201.3 & & & & \\
\hline & SD & 9.7 & & & & \\
\hline & \multirow{35}{*}{\begin{tabular}{|l} 
Individual \\
Data \\
Points
\end{tabular}} & 204 & 194 & 208 & 200 & 202 \\
\hline & & 204 & 196 & 182 & & 206 \\
\hline & & 198 & 192 & 221 & 208 & 196 \\
\hline & & 200 & 196 & 206 & 202 & 196 \\
\hline & & 196 & 208 & 196 & 194 & 194 \\
\hline & & 182 & 208 & 200 & 206 & 206 \\
\hline & & 178 & 214 & 196 & 202 & 202 \\
\hline & & 173 & 202 & 192 & 185 & 210 \\
\hline & & 183 & 206 & 196 & 212 & 202 \\
\hline & & 204 & 190 & 196 & 196 & 212 \\
\hline & & 200 & 198 & 187 & & 235 \\
\hline & & 200 & 200 & 202 & 223 & 210 \\
\hline & & 216 & 210 & 202 & 206 & 210 \\
\hline & & 210 & 208 & 190 & 218 & 206 \\
\hline & & 212 & 190 & 198 & 216 & 206 \\
\hline & & 196 & 206 & 196 & 204 & 210 \\
\hline & & 189 & 194 & 208 & 216 & 204 \\
\hline & & 192 & 200 & 204 & 218 & 214 \\
\hline & & 182 & 208 & 189 & 200 & 223 \\
\hline & & 204 & 202 & 185 & 187 & 188 \\
\hline & & 214 & 200 & 192 & 198 & 194 \\
\hline & & 190 & 192 & 185 & 194 & 202 \\
\hline & & 208 & 192 & 182 & 194 & 198 \\
\hline & & 196 & 210 & 196 & 200 & 196 \\
\hline & & 196 & 206 & 206 & 194 & 218 \\
\hline & & 208 & 198 & 196 & 210 & \\
\hline & & 206 & 194 & 200 & 206 & \\
\hline & & 198 & 208 & 214 & 208 & \\
\hline & & 187 & 204 & 204 & 202 & \\
\hline & & 202 & 198 & 190 & 198 & \\
\hline & & 192 & 218 & 210 & 208 & \\
\hline & & 185 & 208 & 202 & 216 & \\
\hline & & 200 & 204 & 216 & 200 & \\
\hline & & 202 & 202 & 202 & 208 & \\
\hline & & 212 & 208 & 206 & 200 & \\
\hline
\end{tabular}


Table 3.1. Property Values at the Start and End (Peak) of Age-Hardening. The same values for U-5.6Nb and U-7.7Nb used in the previous study [2007hac2] were also used in the present work.

\begin{tabular}{|c|c|c|c|c|}
\hline \multicolumn{2}{|l|}{ Property } & U-5.6Nb & U-7.7Nb & Banded U-6Nb \\
\hline \multirow[t]{2}{*}{$\mathrm{HV}$} & $P$ (start) & 147.2 & 115.2 & 166.7 \\
\hline & $P($ peak $)$ & 600.0 & 600.0 & 600.0 \\
\hline \multirow[t]{2}{*}{ 1YS (MPa) } & $P($ start $)$ & 125.4 & 96.7 & 157.3 \\
\hline & $P($ peak $)$ & $1,200.0$ & $1,400.0$ & $1,200.0$ \\
\hline \multirow[t]{2}{*}{ 2YS (MPa) } & $P($ start $)$ & 545.2 & 509.0 & 655.0 \\
\hline & $P($ peak $)$ & $1,200.0$ & $1,400.0$ & $1,200.0$ \\
\hline \multirow[t]{2}{*}{ UTS (MPa) } & $P$ (start) & 792.0 & 699.0 & 818.7 \\
\hline & $P($ peak $)$ & $1,200.0$ & $1,400.0$ & $1,200.0$ \\
\hline \multirow[t]{2}{*}{ UE } & $P($ start $)$ & 0.2314 & 0.2840 & 0.2382 \\
\hline & $P($ peak $)$ & 0.0000 & 0.0000 & 0.0000 \\
\hline \multirow[t]{2}{*}{ TE } & $P($ start $)$ & 0.2592 & 0.3900 & 0.3920 \\
\hline & $P($ peak $)$ & 0.0000 & 0.0000 & 0.0000 \\
\hline \multirow[t]{2}{*}{$\%$ RA } & $P($ start $)$ & 32.95 & 56.97 & 39.11 \\
\hline & $P($ peak $)$ & 0.00 & 0.00 & 0.00 \\
\hline \multirow{2}{*}{ 1YM (MPa) } & $P($ start $)$ & 52,500 & 31,000 & 53,333 \\
\hline & $P($ peak $)$ & 120,000 & 85,000 & 120,000 \\
\hline
\end{tabular}


Table 3.2. Kinetic Parameters for U-5.6Nb Obtained from the Previous Study [2007hac2]. The $Q$ values were fit at $\boldsymbol{f}=0.25$ using data from all 4 temperatures.

\begin{tabular}{|c|l|r|r|r|r|r|r|r|r|}
\hline$P$ & $\begin{array}{l}Q \\
\text { (kcal } \\
\text { /mol) }\end{array}$ & \multicolumn{3}{|c|}{$x_{o}$ value at the indicated temperature } & \multicolumn{3}{|c|}{$d x$ value at the indicated temperature } \\
\cline { 3 - 10 } & $100^{\circ} \mathrm{C}$ & $200^{\circ} \mathrm{C}$ & $250^{\circ} \mathrm{C}$ & $300^{\circ} \mathrm{C}$ & $100^{\circ} \mathrm{C}$ & $200^{\circ} \mathrm{C}$ & $250^{\circ} \mathrm{C}$ & $300^{\circ} \mathrm{C}$ \\
\hline HV & 115.922 & 33.2797 & 5.6516 & 4.6900 & 3.3261 & 8.2681 & 1.9506 & 1.7056 & 1.5891 \\
\hline 2YS & 29.069 & 6.8210 & 4.1863 & 3.2708 & 1.3850 & 0.7923 & 1.2408 & 1.9511 & 1.2687 \\
\hline TE & 29.030 & 6.8129 & 3.5431 & 2.2016 & 0.8536 & 0.9853 & 0.9450 & 1.4430 & 0.8303 \\
\hline
\end{tabular}

Table 3.3. Kinetic Parameters for U-7.7Nb Obtained from the Previous Study [2007hac2]. The $Q$ values were fit at $\boldsymbol{f}=0.25$ using data from all 4 temperatures.

\begin{tabular}{|c|l|r|r|r|r|r|r|r|r|}
\hline$P$ & $\begin{array}{l}Q \\
(\mathrm{kcal} \\
\text { /mol })\end{array}$ & \multicolumn{2}{|c|}{$x_{o}$ value at the indicated temperature } & \multicolumn{3}{|c|}{$d x$ value at the indicated temperature } \\
\cline { 3 - 11 } & $100^{\circ} \mathrm{C}$ & $200^{\circ} \mathrm{C}$ & $250^{\circ} \mathrm{C}$ & $300^{\circ} \mathrm{C}$ & $100^{\circ} \mathrm{C}$ & $200^{\circ} \mathrm{C}$ & $250^{\circ} \mathrm{C}$ & $300^{\circ} \mathrm{C}$ \\
\hline HV & 20.650 & 9.7186 & 6.7170 & 4.7351 & 3.3211 & 3.2504 & 2.3486 & 1.7270 & 1.3380 \\
\hline 2YS & 30.532 & 10.4549 & 5.1036 & 2.6139 & 1.5471 & 3.9061 & 2.0877 & 1.6372 & 1.3549 \\
\hline TE & 25.216 & 6.6042 & 3.5201 & 1.0653 & 1.1052 & 1.1993 & 1.5680 & 0.6342 & 0.5359 \\
\hline
\end{tabular}

Table 3.4. Lifetimes for U-5.6Nb and U-7.7Nb Obtained from the Previous Study [2007hac2], on the Basis of the $f^{\prime} \geq 0.25$ Failure Criterion for TE. The minimum lifetime values for each alloy and storage temperature of the four values calculated are highlighted in bold.

\begin{tabular}{|c|c|c|c|c|c|c|c|c|}
\hline \multirow[t]{2}{*}{$\begin{array}{l}\text { Storage } \\
\text { Temp. }\end{array}$} & \multicolumn{4}{|c|}{$\begin{array}{l}\text { U-5.6Nb lifetime (years) extrapolated } \\
\text { from the column-heading temperature }\end{array}$} & \multicolumn{4}{|c|}{$\begin{array}{l}\text { U-7.7Nb lifetime (years) extrapolated } \\
\text { from the column-heading temperature }\end{array}$} \\
\hline & $100^{\circ} \mathrm{C}$ & $200^{\circ} \mathrm{C}$ & $250^{\circ} \mathrm{C}$ & $300^{\circ} \mathrm{C}$ & $100^{\circ} \mathrm{C}$ & $200^{\circ} \mathrm{C}$ & $250^{\circ} \mathrm{C}$ & $300^{\circ} \mathrm{C}$ \\
\hline 30 & & & & & & & 362 & 3,815 \\
\hline & & & & & & & & 1,004 \\
\hline $50^{\circ} \mathrm{C}$ & 1,835 & 3,989 & $2,03 /$ & 1,998 & 236 & 145 & 27 & 287 \\
\hline
\end{tabular}


Table 3.5. Analytic Method Comparison between the Previous and Present Studies.

\begin{tabular}{|c|c|c|c|}
\hline Topic & Characteristic & Previous Study & Present Study \\
\hline \multirow[t]{2}{*}{$\begin{array}{l}\text { Experimental } \\
\text { data set }\end{array}$} & $\begin{array}{l}\text { For model } \\
\text { fitting }\end{array}$ & $\begin{array}{l}\text { Nonbanded U-5.6Nb and U- } \\
7.7 \mathrm{Nb} \text { aged } 100^{\circ} \mathrm{C}, 200^{\circ} \mathrm{C} \text {, } \\
250^{\circ} \mathrm{C}, 300^{\circ} \mathrm{C} \text { up to } 140 \text { days. }\end{array}$ & Same \\
\hline & For validation & None & $\begin{array}{l}\text { 1) Nonbanded } \mathrm{U}-5.6 \mathrm{Nb} \text { and } \mathrm{U}- \\
7.7 \mathrm{Nb} \text { aged } 100^{\circ} \mathrm{C}, 200^{\circ} \mathrm{C} \text {, } \\
300^{\circ} \mathrm{C} \text { for } 221 \text { days. } \\
\text { 2) Banded } \mathrm{U}-6 \mathrm{Nb} \text { aged } 40^{\circ} \mathrm{C} \text {, } \\
65^{\circ} \mathrm{C}, 90^{\circ} \mathrm{C} \text { for } 5 \text { years. }\end{array}$ \\
\hline \multirow{2}{*}{$\begin{array}{l}\text { Fraction } \\
\text { transformed }\end{array}$} & Formula & Eq. 3.1 & Same \\
\hline & $\begin{array}{l}\text { Normalization } \\
\text { values } \\
P(\text { start }) \text { and } \\
P(\text { peak })\end{array}$ & Table 3.1 & Same \\
\hline \multirow[t]{5}{*}{ Model fitting } & $\begin{array}{l}\text { Data form and } \\
\text { weighting }\end{array}$ & Averages, weighted by $S D$ & $\begin{array}{l}\text { All replicate data points, } \\
\text { equally weighted }\end{array}$ \\
\hline & $\begin{array}{l}\text { Analyst } \\
\text { interventions }\end{array}$ & $\begin{array}{l}\text { Fictitious long-time points } \\
\text { added to guide } 100^{\circ} \mathrm{C} \text { and } \\
200^{\circ} \mathrm{C} \text { time-series UE and TE }\end{array}$ & None \\
\hline & $\begin{array}{l}\text { Time-series } \\
\text { function }\end{array}$ & $\begin{array}{l}\text { Boltzmann; two adjustable } \\
\text { parameters } x_{o} \text { and } d x\end{array}$ & $\begin{array}{l}\text { Logistic, two adjustable } \\
\text { parameters } A(=1 / d x) \text { and } B(= \\
\left.x_{o}\right) \text {; equivalent to Boltzmann }\end{array}$ \\
\hline & $\begin{array}{l}\text { Temperature- } \\
\text { series } \\
\text { function } \\
\end{array}$ & Arrhenius & Same \\
\hline & $\begin{array}{l}\text { Fitting } \\
\text { method }\end{array}$ & $\begin{array}{l}\text { Sequential time, then temp.: } \\
\text { 1) Time-series: Nonlinear } \\
\text { least-squares fitting of } x_{o} \text { and } \\
d x \text {. Both parameters were } \\
\text { specific to each temperature } \\
\text { and were unconstrained. } \\
\text { 2) Arrhenius fitting for } Q \text { from } \\
\text { linear fit to log(time to reach } f \\
=0.25) \text { vs } 1 / T \text {. }\end{array}$ & $\begin{array}{l}\text { Simultaneous time and temp.: } \\
\text { Nonlinear least-squares fitting } \\
\text { of } A, B \text {, and } Q \text {. } A \text { was } \\
\text { constrained to have the same } \\
\text { value for all temperatures } \\
\text { (making it isokinetic). } A, B \text {, } \\
\text { and } Q \text { were further forced to } \\
\text { be "perfectly-Arrhenius.” }\end{array}$ \\
\hline \multirow[t]{2}{*}{$\begin{array}{l}\text { Lifetime } \\
\text { prediction }\end{array}$} & $\begin{array}{l}\text { Failure } \\
\text { criterion }\end{array}$ & $\begin{array}{l}f^{\prime} \geq 0.25 \text { for TE; equivalently, } \\
f \geq 0.3866(\mathrm{U}-5.6 \mathrm{Nb}) \text { or } \\
f \geq 0.3404(\mathrm{U}-7.7 \mathrm{Nb})\end{array}$ & Same \\
\hline & $\begin{array}{l}\text { Lifetime } \\
\text { evaluation }\end{array}$ & $\begin{array}{l}\text { Lifetime spread from } \\
\text { minimum and maximum } \\
\text { values from extrapolations } \\
\text { from each of the four aging } \\
\text { temperature fits. }\end{array}$ & $\begin{array}{l}\text { Extrapolations from each of } \\
\text { the four aging temperature fits } \\
\text { resulted in a single value (from } \\
\text { "perfect-Arrhenius” forcing). } \\
\text { Lifetime spread from } 95 \% \text { CIs. }\end{array}$ \\
\hline $\begin{array}{l}\text { Model } \\
\text { validation }\end{array}$ & & None & $\begin{array}{l}\text { 1a) U-5.6Nb: HV only } \\
\text { 1b) U-7.7Nb: HV and tensile } \\
\text { 2) U-6Nb: HV and tensile }\end{array}$ \\
\hline
\end{tabular}


Table 3.6. Total Elongation Kinetic Parameters for U-5.6Nb. The $t$ value used to compute the confidence interval is 2.018 . The units of $Q$ are $\mathrm{kcal} / \mathrm{mol} ; A$ and $B$ were assessed on the basis of the time in minutes.

\begin{tabular}{|l|r|r|r|r|}
\hline \multicolumn{5}{|c|}{ U-5.6Nb TE } \\
\hline Parameter & Estimate & Standard Error & \multicolumn{2}{|c|}{ 95\% Confidence Interval } \\
\cline { 4 - 5 } & & & Lower Bound & \multicolumn{1}{c|}{ Upper Bound } \\
\hline$Q$ & 31.699 & 4.985 & 21.639 & 41.759 \\
\hline$A$ & 0.9666 & 0.1734 & 0.6167 & 1.3165 \\
\hline$B$ & 0.9955 & 0.3043 & 0.3814 & 1.6096 \\
\hline
\end{tabular}

Table 3.7. Total Elongation Kinetic Parameters for U-7.7Nb. The $t$ value used to compute the confidence interval is 2.028 . The units of $Q$ are $\mathrm{kcal} / \mathrm{mol}$; $A$ and $B$ were assessed on the basis of the time in minutes.

\begin{tabular}{|l|r|r|r|r|}
\hline \multicolumn{5}{|c|}{ U-7.7Nb TE } \\
\hline Parameter & Estimate & Standard Error & \multicolumn{2}{|c|}{ 95\% Confidence Interval } \\
\cline { 4 - 5 } & & & \multicolumn{1}{c|}{ Lower Bound } & Upper Bound \\
\hline$Q$ & 39.286 & 6.285 & 26.540 & 52.032 \\
\hline$A$ & 0.6577 & 0.1225 & 0.4093 & 0.9061 \\
\hline$B$ & -0.1346 & 0.4805 & -1.1091 & 0.8399 \\
\hline
\end{tabular}

Table 3.8. Uniform Elongation Kinetic Parameters for U-5.6Nb. The $t$ value used to compute the confidence interval is 2.018 . The units of $Q$ are $\mathrm{kcal} / \mathrm{mol} ; A$ and $B$ were assessed on the basis of the time in minutes.

\begin{tabular}{|l|r|r|r|r|}
\hline \multicolumn{5}{|c|}{ U-5.6Nb UE } \\
\hline Parameter & Estimate & Standard Error & \multicolumn{2}{c|}{ 95\% Confidence Interval } \\
\cline { 4 - 5 } & & & Lower Bound & Upper Bound \\
\hline$Q$ & 31.836 & 4.306 & 22.696 & 40.526 \\
\hline$A$ & 1.0612 & 0.1680 & 0.7222 & 1.4002 \\
\hline$B$ & 1.1163 & 0.2514 & 0.6090 & 1.6236 \\
\hline
\end{tabular}

Table 3.9. Uniform Elongation Kinetic Parameters for U-7.7Nb. The $t$ value used to compute the confidence interval is 2.028 . The units of $Q$ are $\mathrm{kcal} / \mathrm{mol} ; A$ and $B$ were assessed on the basis of the time in minutes.

\begin{tabular}{|l|r|r|r|r|}
\hline \multicolumn{5}{|c|}{ U-7.7Nb UE } \\
\hline Parameter & Estimate & Standard Error & \multicolumn{2}{|c|}{ 95\% Confidence Interval } \\
\cline { 4 - 5 } & & & Lower Bound & \multicolumn{1}{c|}{ Upper Bound } \\
\hline$Q$ & 51.741 & 6.415 & 38.731 & 64.751 \\
\hline$A$ & 0.9927 & 0.1637 & 0.6607 & 1.3247 \\
\hline$B$ & -0.6659 & 0.4295 & -1.5369 & 0.2051 \\
\hline
\end{tabular}


Table 3.10. First-Yield Strength Kinetic Parameters for U-5.6Nb. The $t$ value used to compute the confidence interval is 2.018 . The units of $Q$ are $\mathrm{kcal} / \mathrm{mol} ; A$ and $B$ were assessed on the basis of the time in minutes.

\begin{tabular}{|l|r|r|r|r|}
\hline \multicolumn{5}{|c|}{ U-5.6Nb 1YS } \\
\hline Parameter & \multirow{2}{*}{ Estimate } & Standard Error & \multicolumn{2}{|c|}{ 95\% Confidence Interval } \\
\cline { 4 - 5 } & & & \multicolumn{1}{c|}{ Lower Bound } & \multicolumn{1}{c|}{ Upper Bound } \\
\hline$Q$ & 20.813 & 1.796 & 17.189 & 24.437 \\
\hline$A$ & 1.1425 & 0.0956 & 0.9496 & 1.3354 \\
\hline$B$ & 2.4264 & 0.1132 & 2.1980 & 2.6548 \\
\hline
\end{tabular}

Table 3.11. First-Yield Strength Kinetic Parameters for U-7.7Nb. The $t$ value used to compute the confidence interval is 2.028 . The units of $Q$ are $\mathrm{kcal} / \mathrm{mol} ; A$ and $B$ were assessed on the basis of the time in minutes.

\begin{tabular}{|l|r|r|r|r|}
\hline \multicolumn{5}{|c|}{ U-7.7Nb 1YS } \\
\hline$Q$ & Estimate & Standard Error & \multicolumn{2}{|c|}{ 95\% Confidence Interval } \\
\cline { 4 - 5 } & & & Lower Bound & \multicolumn{1}{c|}{ Upper Bound } \\
\hline$A$ & 15.243 & 2.204 & 10.773 & 19.713 \\
\hline$B$ & 0.9190 & 0.1252 & 0.6651 & 1.1729 \\
\hline
\end{tabular}

Table 3.12. First-Yield Modulus Kinetic Parameters for U-5.6Nb. The $t$ value used to compute the confidence interval is 2.018 . The units of $Q$ are $\mathrm{kcal} / \mathrm{mol} ; A$ and $B$ were assessed on the basis of the time in minutes.

\begin{tabular}{|l|r|r|r|r|}
\hline \multicolumn{5}{|c|}{ U-5.6Nb 1YM } \\
\hline Parameter & Estimate & Standard Error & \multicolumn{2}{|c|}{ 95\% Confidence Interval } \\
\cline { 3 - 5 } & & & \multicolumn{1}{c|}{ Lower Bound } & \multicolumn{1}{c|}{ Upper Bound } \\
\hline$Q$ & 14.259 & 4.084 & 6.017 & 22.501 \\
\hline$A$ & 0.7710 & 0.1823 & 0.4031 & 1.1389 \\
\hline$B$ & 2.5672 & 0.3672 & 1.8262 & 3.3082 \\
\hline
\end{tabular}

Table 3.13. First-Yield Modulus Kinetic Parameters for U-7.7Nb. The $t$ value used to compute the confidence interval is 2.028 . The units of $Q$ are $\mathrm{kcal} / \mathrm{mol} ; A$ and $B$ were assessed on the basis of the time in minutes.

\begin{tabular}{|l|r|r|r|r|}
\hline \multicolumn{5}{|c|}{ U-7.7Nb 1YM } \\
\hline Parameter & Estimate & Standard Error & \multicolumn{2}{|c|}{ 95\% Confidence Interval } \\
\cline { 3 - 5 } & & & Lower Bound & \multicolumn{1}{c|}{ Upper Bound } \\
\hline$Q$ & 13.655 & 2.312 & 8.966 & 18.344 \\
\hline$A$ & 1.0966 & 0.1890 & 0.7133 & 1.4799 \\
\hline$B$ & 2.8964 & 0.2051 & 2.4805 & 3.3123 \\
\hline
\end{tabular}


Table 3.14. Second-Yield Strength Kinetic Parameters for U-5.6Nb. The $t$ value used to compute the confidence interval is 2.018 . The units of $Q$ are $\mathrm{kcal} / \mathrm{mol}$; $A$ and $B$ were assessed on the basis of the time in minutes.

\begin{tabular}{|l|r|r|r|r|}
\hline \multicolumn{5}{|c|}{ U-5.6Nb 2YS } \\
\hline Parameter & Estimate & Standard Error & \multicolumn{2}{|c|}{ 95\% Confidence Interval } \\
\cline { 4 - 5 } & & & Lower Bound & Upper Bound \\
\hline$Q$ & 34.178 & 2.570 & 28.992 & 39.364 \\
\hline$A$ & 0.8244 & 0.0690 & 0.6852 & 0.9636 \\
\hline$B$ & 1.4401 & 0.1444 & 1.1488 & 1.7315 \\
\hline
\end{tabular}

Table 3.15. Second-Yield Strength Kinetic Parameters for U-7.7Nb. The $t$ value used to compute the confidence interval is 2.028 . The units of $Q$ are $\mathrm{kcal} / \mathrm{mol} ; A$ and $B$ were assessed on the basis of the time in minutes.

\begin{tabular}{|l|r|r|r|r|}
\hline \multicolumn{5}{|c|}{ U-7.7Nb 2YS } \\
\hline Parameter & Estimate & Standard Error & \multicolumn{2}{|c|}{$95 \%$ Confidence Interval } \\
\cline { 4 - 5 } & & & Lower Bound & Upper Bound \\
\hline$Q$ & 33.927 & 3.038 & 27.766 & 40.088 \\
\hline$A$ & 0.4981 & 0.0458 & 0.4052 & 0.5910 \\
\hline$B$ & 1.6405 & 0.1751 & 1.2854 & 1.9956 \\
\hline
\end{tabular}

Table 3.16. Ultimate Tensile Strength Kinetic Parameters for U-5.6Nb. The $t$ value used to compute the confidence interval is 2.018 . The units of $Q$ are $\mathrm{kcal} / \mathrm{mol} ; A$ and $B$ were assessed on the basis of the time in minutes.

\begin{tabular}{|l|r|r|r|r|}
\hline \multicolumn{5}{|c|}{ U-5.6Nb UTS } \\
\hline Parameter & Estimate & Standard Error & \multicolumn{2}{|c|}{ 95\% Confidence Interval } \\
\cline { 4 - 5 } & & & Lower Bound & Upper Bound \\
\hline$Q$ & 41.365 & 4.984 & 31.307 & 51.423 \\
\hline$A$ & 0.8555 & 0.1444 & 0.5641 & 1.1469 \\
\hline$B$ & 1.8603 & 0.2418 & 1.3723 & 2.3483 \\
\hline
\end{tabular}

Table 3.17. Ultimate Tensile Strength Kinetic Parameters for U-7.7Nb. The $t$ value used to compute the confidence interval is 2.028 . The units of $Q$ are $\mathrm{kcal} / \mathrm{mol} ; A$ and $B$ were assessed on the basis of the time in minutes.

\begin{tabular}{|l|r|r|r|r|}
\hline \multicolumn{5}{|c|}{ U-7.7Nb UTS } \\
\hline Parameter & Estimate & Standard Error & \multicolumn{2}{|c|}{ 95\% Confidence Interval } \\
\cline { 4 - 5 } & & & Lower Bound & Upper Bound \\
\hline$Q$ & 46.146 & 2.593 & 40.887 & 51.405 \\
\hline$A$ & 0.7636 & 0.0538 & 0.6545 & 0.8727 \\
\hline$B$ & 1.7249 & 0.1116 & 1.4986 & 1.9512 \\
\hline
\end{tabular}


Table 3.18. Vickers Hardness Kinetic Parameters for U-5.6Nb. The $t$ value used to compute the confidence interval is 2.110 . The units of $Q$ are $\mathrm{kcal} / \mathrm{mol} ; A$ and $B$ were assessed on the basis of the time in minutes.

\begin{tabular}{|l|r|r|r|r|}
\hline \multicolumn{5}{|c|}{ U-5.6Nb HV } \\
\hline Parameter & Estimate & Standard Error & \multicolumn{2}{|c|}{ 95\% Confidence Interval } \\
\cline { 4 - 5 } & & & Lower Bound & Upper Bound \\
\hline$Q$ & 27.734 & 2.263 & 22.959 & 32.509 \\
\hline$A$ & 0.6136 & 0.0470 & 0.5144 & 0.7128 \\
\hline$B$ & 3.4006 & 0.1141 & 3.1598 & 3.6414 \\
\hline
\end{tabular}

Table 3.19. Vickers Hardness Kinetic Parameters for U-7.7Nb. The $t$ value used to compute the confidence interval is 2.131 . The units of $Q$ are $\mathrm{kcal} / \mathrm{mol}$; $A$ and $B$ were assessed on the basis of the time in minutes.

\begin{tabular}{|l|r|r|r|r|}
\hline \multicolumn{5}{|c|}{ U-7.7Nb HV } \\
\hline Parameter & Estimate & Standard Error & \multicolumn{2}{c|}{ 95\% Confidence Interval } \\
\cline { 4 - 5 } & & & Lower Bound & Upper Bound \\
\hline$Q$ & 22.538 & 4042 & 13.924 & 31.152 \\
\hline$A$ & 0.4763 & 0.0716 & 0.3237 & 0.6289 \\
\hline$B$ & 4.1543 & 0.3261 & 3.4594 & 4.8492 \\
\hline
\end{tabular}


Table 4.1. Results for Three Quality-of-Model-Fitting Metrics Evaluated for each Property in $\mathrm{U}-5.6 \mathrm{Nb}$. The color coding is a redundant indicator of the text result in each box (green $=$ good, yellow $=$ fair, pink $=$ questionable). The number in parentheses in the Goodness of Fit column is the RSE value. The parenthetical comments in the Symmetry of Fit column call out the temperatures where notable asymmetry was found.

\begin{tabular}{|l|l|l|l|}
\hline Property & $\begin{array}{l}\text { Goodness of Fit } \\
\text { (RSE, fractional basis) }\end{array}$ & $\begin{array}{l}\text { Robustness of Fit } \\
\text { (plot of RSS tau) }\end{array}$ & $\begin{array}{l}\text { Symmetry of Fit } \\
\text { (eveness of data point scatter about prediction) }\end{array}$ \\
\hline TE & fair $(0.1696)$ & fair & good \\
\hline UE & fair $(0.1517)$ & fair & good \\
\hline 1YS & good $(0.0851)$ & good & good \\
\hline 1YM & questionable $(0.2410)$ & questionable & good \\
\hline 2YS & good $(0.0763)$ & good & fair (100C below line) \\
\hline UTS & fair $(0.1326)$ & fair & good \\
\hline HV & good $(0.0321)$ & fair & good \\
\hline
\end{tabular}

Table 4.2. Results for Three Quality-of-Model-Fitting Metrics Evaluated for each Property in $\mathrm{U}-7.7 \mathrm{Nb}$. The color coding is a redundant indicator of the text result in each box (green $=$ good, yellow $=$ fair, pink $=$ questionable). The number in parentheses in the Goodness of Fit column is the RSE value. The parenthetical comments in the Symmetry of Fit column call out the temperatures where notable asymmetry was found.

\begin{tabular}{|l|l|l|l|}
\hline Property & $\begin{array}{l}\text { Goodness of Fit } \\
\text { (RSE, fractional basis) }\end{array}$ & $\begin{array}{l}\text { Robustness of Fit } \\
\text { (plot of RSS tau) }\end{array}$ & $\begin{array}{l}\text { Symmetry of Fit } \\
\text { (eveness of data point scatter about prediction) }\end{array}$ \\
\hline TE & fair $(0.1409)$ & questionable & questionable (100C, 250C above line) \\
\hline UE & fair $(0.1233)$ & questionable & questionable (100C above line) \\
\hline 1YS & fair $(0.1210)$ & fair & questionable (100C above line, 200C below line) \\
\hline 1YM & fair $(0.1631)$ & questionable & questionable (100C above line, 200C below line) \\
\hline 2YS & good $(0.0657)$ & fair & questionable (100C above line, 200C below line) \\
\hline UTS & good $(0.0533)$ & good & questionable (100C above line) \\
\hline HV & good $(0.0614)$ & questionable & questionable (100C above line, 200C below line) \\
\hline
\end{tabular}

Table 4.3. Residual Standard Errors and Ancillary Quantities. The RSE is quoted as an absolute value (MPa units for 1YS, 1YM, 2YS, and UTS) and on the basis of fractional property change. The difference quantity $n-p$ is the degrees of freedom $(d f)$.

\begin{tabular}{|c|c|c|c|c|c|c|c|c|c|c|}
\hline \multirow[t]{3}{*}{ Property } & \multicolumn{5}{|l|}{ U-5.6Nb } & \multicolumn{5}{|l|}{ U-7.7Nb } \\
\hline & \multicolumn{2}{|l|}{$R S E$} & \multirow[t]{2}{*}{$n$} & \multirow[t]{2}{*}{$p$} & \multirow[t]{2}{*}{$n-p$} & \multicolumn{2}{|l|}{$R S E$} & \multirow[t]{2}{*}{$n$} & \multirow[t]{2}{*}{$p$} & \multirow[t]{2}{*}{$n-p$} \\
\hline & fractional & absolute & & & & fractional & absolute & & & \\
\hline $\mathrm{TE}$ & 0.1696 & 0.043964 & 45 & 3 & 42 & 0.1409 & 0.054955 & 39 & 3 & 36 \\
\hline UE & 0.1517 & 0.035097 & 45 & 3 & 42 & 0.1233 & 0.035004 & 39 & 3 & 36 \\
\hline $1 Y S$ & 0.0851 & 91.4812 & 45 & 3 & 42 & 0.1210 & 157.638 & 39 & 3 & 36 \\
\hline 1YM & 0.2410 & 16271 & 45 & 3 & 42 & 0.1631 & 8808.38 & 39 & 3 & 36 \\
\hline $2 Y S$ & 0.0763 & 49.9509 & 45 & 3 & 42 & 0.0657 & 58.5185 & 39 & 3 & 36 \\
\hline UTS & 0.1326 & 54.1052 & 45 & 3 & 42 & 0.0533 & 37.3853 & 39 & 3 & 36 \\
\hline $\mathrm{HV}$ & 0.0321 & 14.5191 & 20 & 3 & 17 & 0.0614 & 29.7493 & 18 & 3 & 15 \\
\hline
\end{tabular}


Table 4.4. Summary of Activation Energies $Q$ from Model Fitting of the Previous Study [2007hac2] and This Study.

\begin{tabular}{|l|c|c|c|c|}
\hline \multirow{2}{*}{ Property } & \multicolumn{4}{|c|}{ Activation Energy Q (kcal/mol) } \\
\cline { 2 - 5 } & \multicolumn{2}{|c|}{ U-5.6Nb } & \multicolumn{2}{c|}{ U-7.7Nb } \\
\cline { 2 - 5 } & $\begin{array}{c}\text { Previous } \\
\text { Study }\end{array}$ & $\begin{array}{c}\text { This } \\
\text { Study }\end{array}$ & $\begin{array}{c}\text { Previous } \\
\text { Study }\end{array}$ & $\begin{array}{c}\text { This } \\
\text { Study }\end{array}$ \\
\hline TE & 29.030 & 31.699 & 25.216 & 39.286 \\
\hline UE & & 31.836 & & 51.741 \\
\hline 1YS & & 20.813 & & 15.243 \\
\hline 1YM & & 14.259 & & 13.655 \\
\hline 2YS & 29.069 & 34.178 & 30.532 & 33.927 \\
\hline UTS & & 41.365 & & 46.146 \\
\hline HV & 115.922 & 27.734 & 20.650 & 22.538 \\
\hline
\end{tabular}

Table 4.5. Summary of A Parameter (= 1/dx) from Model Fitting of the Previous Study [2007hac2] and This Study. The value listed for the previous study is the arithmetically averaged value of the reciprocals of the 4 individual $d x$ values provided in Tables 3.2 and 3.3.

\begin{tabular}{|l|c|c|c|c|}
\hline \multirow{2}{*}{ Property } & \multicolumn{4}{|c|}{ A Parameter } \\
\cline { 2 - 5 } & \multicolumn{2}{|c|}{ U-5.6Nb } & \multicolumn{2}{c|}{ U-7.7Nb } \\
\cline { 2 - 5 } & $\begin{array}{c}\text { Previous } \\
\text { Study }\end{array}$ & $\begin{array}{c}\text { This } \\
\text { Study }\end{array}$ & $\begin{array}{c}\text { Previous } \\
\text { Study }\end{array}$ & $\begin{array}{c}\text { This } \\
\text { Study }\end{array}$ \\
\hline TE & 0.9926 & 0.9666 & 1.2285 & 0.6577 \\
\hline UE & & 1.0612 & & 0.9927 \\
\hline 1YS & & 1.1425 & & 0.9190 \\
\hline 1YM & & 0.7710 & & 1.0966 \\
\hline 2YS & 0.8422 & 0.8244 & 0.5210 & 0.4981 \\
\hline UTS & & 0.8555 & & 0.7636 \\
\hline HV & 0.4623 & 0.6136 & 0.5150 & 0.4763 \\
\hline
\end{tabular}

Table 4.6. Summary of B Parameter $\left(=x_{0}\right.$ at $\left.300^{\circ} \mathrm{C}\right)$ from Model Fitting of the Previous Study [2007hac2] and This Study. The value listed for the previous study is the arithmetically averaged value of the 4 individual values of $x_{o}$, provided in Tables 3.2 and 3.3, after rescaling to $300^{\circ} \mathrm{C}$.

\begin{tabular}{|l|c|c|c|c|}
\hline \multirow{2}{*}{ Property } & \multicolumn{4}{|c|}{ B Parameter } \\
\cline { 2 - 5 } & \multicolumn{2}{|c|}{ U-5.6Nb } & \multicolumn{2}{c|}{ U-7.7Nb } \\
\cline { 2 - 5 } & $\begin{array}{c}\text { Previous } \\
\text { Study }\end{array}$ & $\begin{array}{c}\text { This } \\
\text { Study }\end{array}$ & $\begin{array}{c}\text { Previous } \\
\text { Study }\end{array}$ & $\begin{array}{c}\text { This } \\
\text { Study }\end{array}$ \\
\hline TE & 1.0235 & 0.9955 & 1.0504 & -0.1346 \\
\hline UE & & 1.1163 & & -0.6659 \\
\hline 1YS & & 2.4264 & & 3.0692 \\
\hline 1YM & & 2.5672 & & 2.8964 \\
\hline 2YS & 1.5865 & 1.4401 & 2.9066 & 1.6405 \\
\hline UTS & & 1.8603 & & 1.7249 \\
\hline HV & 9.4076 & 3.4006 & 4.0997 & 4.1543 \\
\hline
\end{tabular}


Table 4.7. Experimentally Measured and Predicted Mean TE Values for Various U-Nb Alloys for Current and Future Model Validation. The $95 \%$ prediction and confidence intervals are also given. The alloy identified as $6 \mathrm{wt} \% \mathrm{Nb}$ is the RFP banded U-6Nb; the alloys identified as $5.6 \mathrm{wt} \%$ and $7.7 \mathrm{wt} \%$ are nonbanded. "NP" indicates that no data point is expected, because of material limitations.

\begin{tabular}{|c|c|c|c|c|c|c|c|c|c|}
\hline \multirow{3}{*}{\begin{tabular}{|l|} 
Alloy \\
Wt.\% \\
Nb
\end{tabular}} & \multirow{3}{*}{$\begin{array}{l}\text { Aging } \\
\text { Temp. } \\
\text { (C) }\end{array}$} & \multirow{2}{*}{\multicolumn{2}{|c|}{$\begin{array}{l}\text { Aging } \\
\text { Time }\end{array}$}} & \multicolumn{6}{|c|}{ Total Plastic Elongation } \\
\hline & & & & \multirow{2}{*}{$\begin{array}{l}\text { Experimental } \\
\text { Mean }\end{array}$} & \multicolumn{2}{|c|}{ Prediction Interval } & \multirow{2}{*}{\begin{tabular}{|l} 
Prediction \\
Mean
\end{tabular}} & \multicolumn{2}{|c|}{ Confidence Interval } \\
\hline & & $(\min )$. & (years) & & Lower & Upper & & Lower & Upper \\
\hline 6 & 40 & 328320 & 0.625 & & 0.3451 & 0.3920 & 0.3901 & 0.3850 & 0.3920 \\
\hline 6 & 40 & 656640 & 1.25 & & 0.3442 & 0.3920 & 0.3894 & 0.3828 & 0.3920 \\
\hline 6 & 40 & 1311840 & 2.5 & & 0.3430 & 0.3920 & 0.3886 & 0.3799 & 0.3920 \\
\hline 6 & 40 & 2629440 & 5.0 & 0.3823 & 0.3413 & 0.3920 & 0.3874 & 0.3760 & 0.3920 \\
\hline 6 & 40 & 5260320 & 10.0 & & 0.3387 & 0.3920 & 0.3859 & 0.3709 & 0.3920 \\
\hline 6 & 40 & 10519200 & 20.0 & & 0.3350 & 0.3920 & 0.3839 & 0.3642 & 0.3920 \\
\hline 6 & 40 & 21038400 & 40.0 & & 0.3296 & 0.3920 & 0.3813 & 0.3555 & 0.3920 \\
\hline 6 & 65 & 328320 & 0.625 & & 0.3345 & 0.3920 & 0.3829 & 0.3644 & 0.3920 \\
\hline 6 & 65 & 656640 & 1.25 & & 0.3291 & 0.3920 & 0.3799 & 0.3559 & 0.3920 \\
\hline 6 & 65 & 1311840 & 2.5 & & 0.3215 & 0.3920 & 0.3760 & 0.3449 & 0.3920 \\
\hline 6 & 65 & 2629440 & 5.0 & 0.3805 & 0.3108 & 0.3920 & 0.3709 & 0.3308 & 0.3920 \\
\hline 6 & 65 & 5260320 & 10.0 & & 0.2961 & 0.3920 & 0.3642 & 0.3128 & 0.3920 \\
\hline 6 & 65 & 10519200 & 20.0 & & 0.2766 & 0.3920 & 0.3557 & 0.2904 & 0.3920 \\
\hline 6 & 65 & 21038400 & 40.0 & & 0.2515 & 0.3920 & 0.3449 & 0.2629 & 0.3920 \\
\hline 6 & 90 & 328320 & 0.625 & & 0.2923 & 0.3920 & 0.3587 & 0.3096 & 0.3920 \\
\hline 6 & 90 & 656640 & 1.25 & & 0.2727 & 0.3920 & 0.3487 & 0.2872 & 0.3920 \\
\hline 6 & 90 & 1311840 & 2.5 & & 0.2480 & 0.3920 & 0.3362 & 0.2602 & 0.3920 \\
\hline 6 & 90 & 2629440 & 5.0 & 0.4054 & 0.2180 & 0.3920 & 0.3207 & 0.2283 & 0.3920 \\
\hline 6 & 90 & 5260320 & 10.0 & & 0.1833 & 0.3920 & 0.3021 & 0.1921 & 0.3920 \\
\hline 6 & 90 & 10519200 & 20.0 & & 0.1450 & 0.3920 & 0.2804 & 0.1526 & 0.3920 \\
\hline 6 & 90 & 21038400 & 40.0 & & 0.1051 & 0.3920 & 0.2558 & 0.1119 & 0.3920 \\
\hline & & & & & & & & & \\
\hline 5.6 & 100 & 318000 & 0.6 & NP & & & 0.2254 & 0.1827 & 0.2592 \\
\hline 5.6 & 100 & 1052400 & 2.0 & & 0.1335 & 0.2592 & 0.2077 & 0.1482 & 0.2592 \\
\hline 5.6 & 100 & 3153600 & 6.0 & & 0.1043 & 0.2592 & 0.1861 & 0.1099 & 0.2592 \\
\hline 5.6 & 200 & 318000 & $\overline{0.6}$ & NP & & & 0.0340 & 0.0082 & 0.0598 \\
\hline 5.6 & 200 & 1052400 & 2.0 & $\mathrm{NP}$ & & & 0.0217 & 0.0011 & 0.0423 \\
\hline 5.6 & 200 & 3153600 & 6.0 & NP & & & 0.0141 & 0.0000 & 0.0300 \\
\hline 5.6 & 300 & 318000 & 0.6 & NP & & & 0.0033 & 0.0000 & 0.0081 \\
\hline 5.6 & 300 & 1052400 & 2.0 & NP & & & 0.0020 & 0.0000 & 0.0053 \\
\hline 5.6 & 300 & 3153600 & 6.0 & $N P$ & & & 0.0013 & 0.0000 & 0.0035 \\
\hline & & & & & & & & & \\
\hline 7.7 & 100 & 318000 & 0.6 & 0.3362 & 0.2450 & 0.3900 & 0.3226 & 0.2686 & 0.3766 \\
\hline 7.7 & 100 & 1052400 & 2.0 & & 0.2137 & 0.3890 & 0.3014 & 0.2337 & 0.3690 \\
\hline 7.7 & 100 & 3153600 & 6.0 & & 0.1886 & 0.3676 & 0.2781 & 0.1967 & 0.3595 \\
\hline 7.7 & 200 & 318000 & 0.6 & 0.0782 & 0.0000 & 0.1307 & 0.0640 & 0.0274 & 0.1006 \\
\hline 7.7 & 200 & 1052400 & 2.0 & & 0.0000 & 0.1128 & 0.0477 & 0.0142 & 0.0813 \\
\hline 7.7 & 200 & 3153600 & 6.0 & & 0.0000 & 0.0993 & 0.0361 & 0.0062 & 0.0659 \\
\hline 7.7 & 300 & 318000 & 0.6 & NP & & & 0.0093 & 0.0000 & 0.0190 \\
\hline 7.7 & 300 & 1052400 & 2.0 & NP & & & 0.0067 & 0.0000 & 0.0144 \\
\hline 7.7 & 300 & 3153600 & 6.0 & $\mathrm{NP}$ & & & 0.0049 & 0.0000 & 0.0112 \\
\hline
\end{tabular}


Table 4.8. Experimentally Measured and Predicted Mean UE Values for Various U-Nb Alloys for Current and Future Model Validation. The $95 \%$ prediction and confidence intervals are also given. The alloy identified as $6 \mathrm{wt} \% \mathrm{Nb}$ is the RFP banded U-6Nb; the alloys identified as $5.6 \mathrm{wt} \%$ and $7.7 \mathrm{wt} \%$ are nonbanded. "NP" indicates that no data point is expected, because of material limitations.

\begin{tabular}{|c|c|c|c|c|c|c|c|c|c|}
\hline \multirow{3}{*}{$\begin{array}{l}\text { Alloy } \\
\text { Wt.\% } \\
\mathrm{Nb} \\
\end{array}$} & \multirow{3}{*}{$\begin{array}{l}\text { Aging } \\
\text { Temp. } \\
\text { (C) }\end{array}$} & \multirow{2}{*}{\multicolumn{2}{|c|}{$\begin{array}{l}\text { Aging } \\
\text { Time }\end{array}$}} & \multicolumn{6}{|c|}{ Uniform Plastic Elongation } \\
\hline & & & & \multirow{2}{*}{$\begin{array}{l}\text { Experimental } \\
\text { Mean }\end{array}$} & \multicolumn{2}{|c|}{ Prediction Interval } & \multirow{2}{*}{\begin{tabular}{|l} 
Prediction \\
Mean
\end{tabular}} & \multicolumn{2}{|c|}{ Confidence Interval } \\
\hline & & (min.) & (years) & & Lower & Upper & & Lower & Upper \\
\hline 6 & 40 & 328320 & 0.625 & & 0.2133 & 0.2382 & 0.2376 & 0.2360 & 0.2382 \\
\hline 6 & 40 & 656640 & 1.25 & & 0.2130 & 0.2382 & 0.2374 & 0.2353 & 0.2382 \\
\hline 6 & 40 & 1311840 & 2.5 & & 0.2126 & 0.2382 & 0.2371 & 0.2342 & 0.2382 \\
\hline 6 & 40 & 2629440 & 5.0 & 0.2101 & 0.2121 & 0.2382 & 0.2367 & 0.2328 & 0.2382 \\
\hline 6 & 40 & 5260320 & 10.0 & & 0.2113 & 0.2382 & 0.2361 & 0.2309 & 0.2382 \\
\hline 6 & 40 & 10519200 & 20.0 & & 0.2100 & 0.2382 & 0.2353 & 0.2283 & 0.2382 \\
\hline 6 & 40 & 21038400 & 40.0 & & 0.2082 & 0.2382 & 0.2343 & 0.2247 & 0.2382 \\
\hline 6 & 65 & 328320 & 0.625 & & 0.2097 & 0.2382 & 0.2349 & 0.2281 & 0.2382 \\
\hline 6 & 65 & 656640 & 1.25 & & 0.2077 & 0.2382 & 0.2337 & 0.2245 & 0.2382 \\
\hline 6 & 65 & 1311840 & 2.5 & & 0.2048 & 0.2382 & 0.2321 & 0.2198 & 0.2382 \\
\hline 6 & 65 & 2629440 & 5.0 & 0.2535 & 0.2005 & 0.2382 & 0.2298 & 0.2135 & 0.2382 \\
\hline 6 & 65 & 5260320 & 10.0 & & 0.1943 & 0.2382 & 0.2268 & 0.2052 & 0.2382 \\
\hline 6 & 65 & 10519200 & 20.0 & & 0.1854 & 0.2382 & 0.2228 & 0.1944 & 0.2382 \\
\hline 6 & 65 & 21038400 & 40.0 & & 0.1732 & 0.2382 & 0.2175 & 0.1805 & 0.2382 \\
\hline 6 & 90 & 328320 & 0.625 & & 0.1917 & 0.2382 & 0.2241 & 0.2026 & 0.2382 \\
\hline 6 & 90 & 656640 & 1.25 & & 0.1822 & 0.2382 & 0.2193 & 0.1913 & 0.2382 \\
\hline 6 & 90 & 1311840 & 2.5 & & 0.1697 & 0.2382 & 0.2129 & 0.1771 & 0.2382 \\
\hline 6 & 90 & 2629440 & 5.0 & 0.2545 & 0.1534 & 0.2382 & 0.2047 & 0.1595 & 0.2382 \\
\hline 6 & 90 & 5260320 & 10.0 & & 0.1335 & 0.2382 & 0.1944 & 0.1386 & 0.2382 \\
\hline 6 & 90 & 10519200 & 20.0 & & 0.1104 & 0.2382 & 0.1819 & 0.1146 & 0.2382 \\
\hline 6 & 90 & 21038400 & 40.0 & & 0.0850 & 0.2382 & 0.1670 & 0.0887 & 0.2382 \\
\hline & & & & & & & & & \\
\hline 5.6 & 100 & 318000 & 0.6 & NP & & & 0.2091 & 0.1797 & 0.2314 \\
\hline 5.6 & 100 & 1052400 & 2.0 & & 0.1391 & 0.2314 & 0.1953 & 0.1517 & 0.2314 \\
\hline 5.6 & 100 & 3153600 & 6.0 & & 0.1135 & 0.2314 & 0.1771 & 0.1180 & 0.2314 \\
\hline 5.6 & 200 & 318000 & $\overline{0.6}$ & NP & & & 0.0292 & 0.0088 & 0.0496 \\
\hline 5.6 & 200 & 1052400 & 2.0 & $\mathrm{NP}$ & & & 0.0178 & 0.0021 & 0.0334 \\
\hline 5.6 & 200 & 3153600 & 6.0 & NP & & & 0.0110 & 0.0000 & 0.0226 \\
\hline 5.6 & 300 & 318000 & 0.6 & NP & & & 0.0022 & 0.0000 & 0.0053 \\
\hline 5.6 & 300 & 1052400 & 2.0 & NP & & & 0.0013 & 0.0000 & 0.0033 \\
\hline 5.6 & 300 & 3153600 & 6.0 & $N P$ & & & 0.0008 & 0.0000 & 0.0021 \\
\hline & & & & & & & & & \\
\hline 7.7 & 100 & 318000 & 0.6 & 0.2488 & 0.2443 & 0.2840 & 0.2804 & 0.2738 & 0.2840 \\
\hline 7.7 & 100 & 1052400 & 2.0 & & 0.2410 & 0.2840 & 0.2780 & 0.2676 & 0.2840 \\
\hline 7.7 & 100 & 3153600 & 6.0 & & 0.2460 & 0.2840 & 0.2746 & 0.2586 & 0.2840 \\
\hline 7.7 & 200 & 318000 & 0.6 & 0.0445 & 0.0000 & 0.0769 & 0.0342 & 0.0104 & 0.0579 \\
\hline 7.7 & 200 & 1052400 & 2.0 & & 0.0000 & 0.0615 & 0.0214 & 0.0028 & 0.0401 \\
\hline 7.7 & 200 & 3153600 & 6.0 & & 0.0000 & 0.0520 & 0.0137 & 0.0000 & 0.0280 \\
\hline 7.7 & 300 & 318000 & 0.6 & NP & & & 0.0006 & 0.0000 & 0.0017 \\
\hline 7.7 & 300 & 1052400 & 2.0 & NP & & & 0.0004 & 0.0000 & 0.0011 \\
\hline 7.7 & 300 & 3153600 & 6.0 & NP & & & 0.0002 & 0.0000 & 0.0007 \\
\hline
\end{tabular}


Table 4.9. Experimentally Measured and Predicted Mean 1YS Values for Various U-Nb Alloys for Current and Future Model Validation. The $95 \%$ prediction and confidence intervals are also given. The alloy identified as $6 \mathrm{wt} \% \mathrm{Nb}$ is the RFP banded $\mathrm{U}-6 \mathrm{Nb}$; the alloys identified as $5.6 \mathrm{wt} \%$ and $7.7 \mathrm{wt} \%$ are nonbanded. "NP" indicates that no data point is expected, because of material limitations.

\begin{tabular}{|c|c|c|c|c|c|c|c|c|c|}
\hline \multirow{3}{*}{$\begin{array}{l}\text { Alloy } \\
\text { Wt.\% } \\
\mathrm{Nb}\end{array}$} & \multirow{3}{*}{$\begin{array}{l}\text { Aging } \\
\text { Temp. } \\
\text { (C) }\end{array}$} & \multirow{2}{*}{\multicolumn{2}{|c|}{$\begin{array}{l}\text { Aging } \\
\text { Time }\end{array}$}} & \multicolumn{6}{|c|}{ First Yield Strength (MPa) } \\
\hline & & & & \multirow{2}{*}{$\begin{array}{l}\text { Experimental } \\
\text { Mean }\end{array}$} & \multicolumn{2}{|c|}{ Prediction Interval } & \multirow{2}{*}{$\begin{array}{l}\text { Prediction } \\
\text { Mean }\end{array}$} & \multicolumn{2}{|c|}{ Confidence Interval } \\
\hline & & $(\min )$. & (years) & & Lower & Upper & & Lower & Upper \\
\hline 6 & 40 & 328320 & 0.625 & & 157.3 & 239.9 & 176.3 & 157.3 & 198.1 \\
\hline 6 & 40 & 656640 & 1.25 & & 157.3 & 250.6 & 183.9 & 157.3 & 213.7 \\
\hline 6 & 40 & 1311840 & 2.5 & & 157.3 & 266.6 & 194.4 & 157.3 & 235.0 \\
\hline 6 & 40 & 2629440 & 5.0 & 195 & 157.3 & 290.1 & 208.9 & 157.3 & 263.9 \\
\hline 6 & 40 & 5260320 & 10.0 & & 157.3 & 323.6 & 228.7 & 157.3 & 302.5 \\
\hline 6 & 40 & 10519200 & 20.0 & & 157.3 & 369.8 & 255.2 & 157.4 & 353.0 \\
\hline 6 & 40 & 21038400 & 40.0 & & 157.3 & 431.0 & 290.3 & 162.8 & 417.7 \\
\hline 6 & 65 & 328320 & 0.625 & & 157.3 & 299.7 & 219.2 & 165.3 & 273.1 \\
\hline 6 & 65 & 656640 & 1.25 & & 157.3 & 335.6 & 242.6 & 171.2 & 314.0 \\
\hline 6 & 65 & 1311840 & 2.5 & & 163.0 & 384.3 & 273.6 & 180.5 & 366.8 \\
\hline 6 & 65 & 2629440 & 5.0 & 266 & 180.9 & 447.8 & 314.3 & 195.0 & 433.7 \\
\hline 6 & 65 & 5260320 & 10.0 & & 205.6 & 526.3 & 365.9 & 217.1 & 514.8 \\
\hline 6 & 65 & 10519200 & 20.0 & & 240.1 & 618.4 & 429.2 & 249.7 & 608.7 \\
\hline 6 & 65 & 21038400 & 40.0 & & 287.7 & 719.8 & 503.8 & 296.1 & 711.4 \\
\hline 6 & 90 & 328320 & 0.625 & & 202.6 & 432.6 & 317.6 & 219.3 & 415.9 \\
\hline 6 & 90 & 656640 & 1.25 & & 234.5 & 505.4 & 370.0 & 248.4 & 491.5 \\
\hline 6 & 90 & 1311840 & 2.5 & & 276.6 & 591.4 & 434.0 & 288.3 & 579.6 \\
\hline 6 & 90 & 2629440 & 5.0 & 396 & 331.3 & 687.6 & 509.5 & 341.7 & 677.3 \\
\hline 6 & 90 & 5260320 & 10.0 & & 399.6 & 787.6 & 593.6 & 409.0 & 778.1 \\
\hline 6 & 90 & 10519200 & 20.0 & & 480.7 & 884.3 & 682.5 & 489.7 & 875.2 \\
\hline 6 & 90 & 21038400 & 40.0 & & 571.1 & 971.1 & 771.1 & 580.2 & 962.0 \\
\hline & & & & & & & & & \\
\hline 5.6 & 100 & 318000 & 0.6 & $\mathrm{NP}$ & & & 348.7 & 232.6 & 464.7 \\
\hline 5.6 & 100 & 1052400 & 2.0 & & 292.2 & 650.6 & 471.4 & 317.8 & 625.0 \\
\hline 5.6 & 100 & 3153600 & 6.0 & & 421.4 & 796.8 & 609.1 & 431.8 & 786.5 \\
\hline 5.6 & 200 & 318000 & 0.6 & NP & & & 1019.6 & 960.8 & 1078.4 \\
\hline 5.6 & 200 & 1052400 & 2.0 & NP & & & 1092.3 & 1045.9 & 1138.7 \\
\hline 5.6 & 200 & 3153600 & 6.0 & NP & & & 1134.8 & 1100.3 & 1169.2 \\
\hline 5.6 & 300 & 318000 & 0.6 & NP & & & 1168.9 & 1149.8 & 1188.0 \\
\hline 5.6 & 300 & 1052400 & 2.0 & NP & & & 1182.6 & 1170.2 & 1195.0 \\
\hline 5.6 & 300 & 3153600 & 6.0 & NP & & & 1189.8 & 1181.7 & 1198.0 \\
\hline & & & & & & & & & \\
\hline 7.7 & 100 & 318000 & 0.6 & 499 & 308.6 & 794.8 & 551.7 & 368.6 & 734.9 \\
\hline 7.7 & 100 & 1052400 & 2.0 & & 438.3 & 963.9 & 701.1 & 492.5 & 909.7 \\
\hline 7.7 & 100 & 3153600 & 6.0 & & 599.0 & 1087.2 & 843.1 & 623.5 & 1062.7 \\
\hline 7.7 & 200 & 318000 & 0.6 & 988 & 879.0 & 1274.3 & 1076.6 & 960.4 & 1192.9 \\
\hline 7.7 & 200 & 1052400 & 2.0 & & 984.8 & 1372.4 & 1178.6 & 1069.0 & 1288.1 \\
\hline 7.7 & 200 & 3153600 & 6.0 & & 1061.9 & 1400.0 & 1247.9 & 1152.8 & 1343.1 \\
\hline 7.7 & 300 & 318000 & 0.6 & NP & & & 1274.2 & 1191.5 & 1356.8 \\
\hline 7.7 & 300 & 1052400 & 2.0 & NP & & & 1319.0 & 1254.6 & 1383.3 \\
\hline 7.7 & 300 & 3153600 & 6.0 & NP & & & 1346.5 & 1297.3 & 1395.7 \\
\hline
\end{tabular}


Table 4.10. Experimentally Measured and Predicted Mean 1YM Values for Various U-Nb Alloys for Current and Future Model Validation. The $95 \%$ prediction and confidence intervals are also given. The alloy identified as $6 \mathrm{wt} \% \mathrm{Nb}$ is the RFP banded U-6Nb; the alloys identified as $5.6 \mathrm{wt} \%$ and $7.7 \mathrm{wt} \%$ are nonbanded. "NP" indicates that no data point is expected, because of material limitations.

\begin{tabular}{|c|c|c|c|c|c|c|c|c|c|}
\hline \multirow{3}{*}{$\begin{array}{l}\text { Alloy } \\
\text { Wt.\% } \\
\mathrm{Nb}\end{array}$} & \multirow{3}{*}{$\begin{array}{l}\text { Aging } \\
\text { Temp. } \\
\text { (C) }\end{array}$} & \multirow{2}{*}{\multicolumn{2}{|c|}{$\begin{array}{l}\text { Aging } \\
\text { Time }\end{array}$}} & \multicolumn{6}{|c|}{ First Yield Modulus (MPa) } \\
\hline & & & & \multirow{2}{*}{$\begin{array}{l}\text { Experimental } \\
\text { Mean }\end{array}$} & \multicolumn{2}{|c|}{ Prediction Interval } & \multirow{2}{*}{$\begin{array}{l}\text { Prediction } \\
\text { Mean }\end{array}$} & \multicolumn{2}{|c|}{ Confidence Interval } \\
\hline & & $(\min )$. & (years) & & Lower & Upper & & Lower & Upper \\
\hline 6 & 40 & 328320 & 0.625 & & 53333 & 89866 & 68742 & 53333 & 86890 \\
\hline 6 & 40 & 656640 & 1.25 & & 53333 & 94825 & 71661 & 53333 & 92148 \\
\hline 6 & 40 & 1311840 & 2.5 & & 53333 & 100079 & 74895 & 53333 & 97641 \\
\hline 6 & 40 & 2629440 & 5.0 & 68333 & 53333 & 105467 & 78421 & 53629 & 103213 \\
\hline 6 & 40 & 5260320 & 10.0 & & 53568 & 110720 & 82144 & 55692 & 108596 \\
\hline 6 & 40 & 10519200 & 20.0 & & 56352 & 115616 & 85984 & 58394 & 113574 \\
\hline 6 & 40 & 21038400 & 40.0 & & 59732 & 119953 & 89843 & 61740 & 117945 \\
\hline 6 & 65 & 328320 & 0.625 & & 54581 & 98256 & 76419 & 57444 & 95393 \\
\hline 6 & 65 & 656640 & 1.25 & & 56823 & 103245 & 80034 & 59494 & 100574 \\
\hline 6 & 65 & 1311840 & 2.5 & & 59477 & 108151 & 83814 & 62010 & 105618 \\
\hline 6 & 65 & 2629440 & 5.0 & 71000 & 62580 & 112794 & 87687 & 65026 & 110348 \\
\hline 6 & 65 & 5260320 & 10.0 & & 66089 & 116956 & 91523 & 68501 & 114545 \\
\hline 6 & 65 & 10519200 & 20.0 & & 69945 & 120000 & 95229 & 72373 & 118085 \\
\hline 6 & 65 & 21038400 & 40.0 & & 74048 & 120000 & 98722 & 76542 & 120000 \\
\hline 6 & 90 & 328320 & 0.625 & & 63646 & 104798 & 84222 & 66714 & 101729 \\
\hline 6 & 90 & 656640 & 1.25 & & 66817 & 109353 & 88085 & 69769 & 106400 \\
\hline 6 & 90 & 1311840 & 2.5 & & 70246 & 113562 & 91904 & 73137 & 110672 \\
\hline 6 & 90 & 2629440 & 5.0 & 83000 & 73907 & 117302 & 95605 & 76792 & 114417 \\
\hline 6 & 90 & 5260320 & 10.0 & & 77698 & 120000 & 99073 & 80633 & 117513 \\
\hline 6 & 90 & 10519200 & 20.0 & & 81526 & 120000 & 102253 & 84568 & 119938 \\
\hline 6 & 90 & 21038400 & 40.0 & & 85287 & 120000 & 105108 & 88494 & 120000 \\
\hline & & & & & & & & & \\
\hline 5.6 & 100 & 318000 & 0.6 & NP & & & 86578 & 69816 & 103340 \\
\hline 5.6 & 100 & 1052400 & 2.0 & & 69175 & 117301 & 93238 & 75646 & 110830 \\
\hline 5.6 & 100 & 3153600 & 6.0 & & 78365 & 119425 & 98895 & 81526 & 116264 \\
\hline 5.6 & 200 & 318000 & 0.6 & NP & & & 106414 & 96596 & 116232 \\
\hline 5.6 & 200 & 1052400 & 2.0 & NP & & & 110252 & 101246 & 119258 \\
\hline 5.6 & 200 & 3153600 & 6.0 & NP & & & 112936 & 105065 & 120000 \\
\hline 5.6 & 300 & 318000 & 0.6 & NP & & & 113640 & 106681 & 120000 \\
\hline 5.6 & 300 & 1052400 & 2.0 & NP & & & 115603 & 109933 & 120000 \\
\hline 5.6 & 300 & 3153600 & 6.0 & NP & & & 116893 & 112326 & 120000 \\
\hline & & & & & & & & & \\
\hline 7.7 & 100 & 318000 & 0.6 & 64500 & 41625 & 69039 & 55332 & 44935 & 65729 \\
\hline 7.7 & 100 & 1052400 & 2.0 & & 48921 & 77001 & 62961 & 52129 & 73793 \\
\hline 7.7 & 100 & 3153600 & 6.0 & & 57538 & 81120 & 69329 & 59152 & 79506 \\
\hline 7.7 & 200 & 318000 & 0.6 & 81500 & 65929 & 85000 & 76320 & 71010 & 81629 \\
\hline 7.7 & 200 & 1052400 & 2.0 & & 69791 & 85000 & 79722 & 75380 & 84064 \\
\hline 7.7 & 200 & 3153600 & 6.0 & & 72211 & 85000 & 81741 & 78419 & 85000 \\
\hline 7.7 & 300 & 318000 & 0.6 & NP & & & 82069 & 79007 & 85000 \\
\hline 7.7 & 300 & 1052400 & 2.0 & NP & & & 83302 & 81183 & 85000 \\
\hline 7.7 & 300 & 3153600 & 6.0 & NP & & & 83980 & 82519 & 85000 \\
\hline
\end{tabular}


Table 4.11. Experimentally Measured and Predicted Mean 2YS Values for Various U-Nb Alloys for Current and Future Model Validation. The $95 \%$ prediction and confidence intervals are also given. The alloy identified as $6 \mathrm{wt} \% \mathrm{Nb}$ is the RFP banded $\mathrm{U}-6 \mathrm{Nb}$; the alloys identified as $5.6 \mathrm{wt} \%$ and $7.7 \mathrm{wt} \%$ are nonbanded. "NP" indicates that no data point is expected, because of material limitations.

\begin{tabular}{|c|c|c|c|c|c|c|c|c|c|}
\hline \multirow{3}{*}{$\begin{array}{l}\text { Alloy } \\
\text { Wt.\% } \\
\mathrm{Nb}\end{array}$} & \multirow{3}{*}{$\begin{array}{l}\text { Aging } \\
\text { Temp. } \\
\text { (C) }\end{array}$} & \multirow{2}{*}{\multicolumn{2}{|c|}{$\begin{array}{l}\text { Aging } \\
\text { Time }\end{array}$}} & \multicolumn{6}{|c|}{ Second Yield Strength (MPa) } \\
\hline & & & & \multirow{2}{*}{$\begin{array}{l}\text { Experimental } \\
\text { Mean }\end{array}$} & \multicolumn{2}{|c|}{ Prediction Interval } & \multirow{2}{*}{$\begin{array}{l}\text { Prediction } \\
\text { Mean }\end{array}$} & \multicolumn{2}{|c|}{ Confidence Interval } \\
\hline & & $(\min )$. & (years) & & Lower & Upper & & Lower & Upper \\
\hline 6 & 40 & 328320 & 0.625 & & 655.0 & 685.2 & 657.1 & 655.0 & 659.7 \\
\hline 6 & 40 & 656640 & 1.25 & & 655.0 & 685.9 & 657.7 & 655.0 & 661.0 \\
\hline 6 & 40 & 1311840 & 2.5 & & 655.0 & 686.7 & 658.5 & 655.0 & 662.6 \\
\hline 6 & 40 & 2629440 & 5.0 & 667 & 655.0 & 687.9 & 659.4 & 655.0 & 664.6 \\
\hline 6 & 40 & 5260320 & 10.0 & & 655.0 & 689.4 & 660.7 & 655.0 & 667.2 \\
\hline 6 & 40 & 10519200 & 20.0 & & 655.0 & 691.4 & 662.3 & 655.0 & 670.5 \\
\hline 6 & 40 & 21038400 & 40.0 & & 655.0 & 694.1 & 664.3 & 655.0 & 674.6 \\
\hline 6 & 65 & 328320 & 0.625 & & 655.0 & 693.2 & 663.9 & 655.4 & 672.4 \\
\hline 6 & 65 & 656640 & 1.25 & & 655.0 & 696.3 & 666.4 & 655.8 & 677.0 \\
\hline 6 & 65 & 1311840 & 2.5 & & 655.0 & 700.4 & 669.5 & 656.3 & 682.7 \\
\hline 6 & 65 & 2629440 & 5.0 & 677 & 655.0 & 705.9 & 673.5 & 656.9 & 690.0 \\
\hline 6 & 65 & 5260320 & 10.0 & & 655.0 & 713.1 & 678.4 & 657.9 & 699.0 \\
\hline 6 & 65 & 10519200 & 20.0 & & 655.0 & 722.5 & 684.7 & 659.2 & 710.1 \\
\hline 6 & 65 & 21038400 & 40.0 & & 655.0 & 734.5 & 692.5 & 661.0 & 723.9 \\
\hline 6 & 90 & 328320 & 0.625 & & 655.0 & 720.1 & 685.0 & 663.7 & 706.2 \\
\hline 6 & 90 & 656640 & 1.25 & & 655.0 & 731.0 & 692.8 & 666.8 & 718.9 \\
\hline 6 & 90 & 1311840 & 2.5 & & 660.3 & 744.8 & 702.5 & 670.9 & 734.2 \\
\hline 6 & 90 & 2629440 & 5.0 & 721 & 667.1 & 762.0 & 714.5 & 676.2 & 752.8 \\
\hline 6 & 90 & 5260320 & 10.0 & & 675.2 & 782.8 & 729.0 & 683.1 & 774.9 \\
\hline 6 & 90 & 10519200 & 20.0 & & 685.2 & 807.6 & 746.4 & 691.9 & 800.8 \\
\hline 6 & 90 & 21038400 & 40.0 & & 697.3 & 836.3 & 766.8 & 703.2 & 830.5 \\
\hline & & & & & & & & & \\
\hline 5.6 & 100 & 318000 & 0.6 & NP & & & 599.6 & 565.9 & 633.3 \\
\hline 5.6 & 100 & 1052400 & 2.0 & & 556.7 & 693.6 & 625.1 & 578.8 & 671.5 \\
\hline 5.6 & 100 & 3153600 & 6.0 & & 587.8 & 726.3 & 657.0 & 596.5 & 717.6 \\
\hline 5.6 & 200 & 318000 & 0.6 & NP & & & 1034.2 & 995.9 & 1072.5 \\
\hline 5.6 & 200 & 1052400 & 2.0 & NP & & & 1081.5 & 1045.8 & 1117.2 \\
\hline 5.6 & 200 & 3153600 & 6.0 & NP & & & 1115.0 & 1083.5 & 1146.5 \\
\hline 5.6 & 300 & 318000 & 0.6 & NP & & & 1177.8 & 1165.8 & 1189.8 \\
\hline 5.6 & 300 & 1052400 & 2.0 & NP & & & 1185.4 & 1176.4 & 1194.3 \\
\hline 5.6 & 300 & 3153600 & 6.0 & NP & & & 1190.0 & 1183.3 & 1196.8 \\
\hline & & & & & & & & & \\
\hline 7.7 & 100 & 318000 & 0.6 & 679 & 589.6 & 746.9 & 668.2 & 616.6 & 719.9 \\
\hline 7.7 & 100 & 1052400 & 2.0 & & 619.7 & 790.2 & 704.9 & 643.7 & 766.2 \\
\hline 7.7 & 100 & 3153600 & 6.0 & & 662.3 & 824.9 & 743.6 & 672.6 & 814.6 \\
\hline 7.7 & 200 & 318000 & 0.6 & 1102 & 1001.2 & 1152.4 & 1076.8 & 1030.0 & 1123.7 \\
\hline 7.7 & 200 & 1052400 & 2.0 & & 1050.0 & 1206.1 & 1128.0 & 1077.4 & 1178.7 \\
\hline 7.7 & 200 & 3153600 & 6.0 & & 1091.6 & 1249.9 & 1170.7 & 1118.4 & 1223.1 \\
\hline 7.7 & 300 & 318000 & 0.6 & NP & & & 1286.4 & 1253.3 & 1319.5 \\
\hline 7.7 & 300 & 1052400 & 2.0 & NP & & & 1309.7 & 1279.1 & 1340.3 \\
\hline 7.7 & 300 & 3153600 & 6.0 & NP & & & 1327.2 & 1299.4 & 1355.1 \\
\hline
\end{tabular}


Table 4.12. Experimentally Measured and Predicted Mean UTS Values for Various U-Nb Alloys for Current and Future Model Validation. The $\mathbf{9 5 \%}$ prediction and confidence intervals are also given. The alloy identified as $6 \mathrm{wt} \% \mathrm{Nb}$ is the RFP banded $\mathrm{U}-6 \mathrm{Nb}$; the alloys identified as $5.6 \mathrm{wt} \%$ and $7.7 \mathrm{wt} \%$ are nonbanded. "NP" indicates that no data point is expected, because of material limitations.

\begin{tabular}{|c|c|c|c|c|c|c|c|c|c|}
\hline \multirow{3}{*}{$\begin{array}{l}\text { Alloy } \\
\text { Wt.\% } \\
\mathrm{Nb}\end{array}$} & \multirow{3}{*}{$\begin{array}{l}\text { Aging } \\
\text { Temp. } \\
\text { (C) }\end{array}$} & \multirow{2}{*}{\multicolumn{2}{|c|}{$\begin{array}{l}\text { Aging } \\
\text { Time }\end{array}$}} & \multicolumn{6}{|c|}{ Ultimate Tensile Strength (MPa) } \\
\hline & & & & \multirow{2}{*}{$\begin{array}{l}\text { Experimental } \\
\text { Mean }\end{array}$} & \multicolumn{2}{|c|}{ Prediction Interval } & \multirow{2}{*}{$\begin{array}{l}\text { Prediction } \\
\text { Mean }\end{array}$} & \multicolumn{2}{|c|}{ Confidence Interval } \\
\hline & & $(\min )$. & (years) & & Lower & Upper & & Lower & JUpper \\
\hline 6 & 40 & 328320 & 0.625 & & 818.7 & 852.8 & 818.8 & 818.7 & 819.2 \\
\hline 6 & 40 & 656640 & 1.25 & & 818.7 & 852.9 & 818.9 & 818.7 & 819.3 \\
\hline 6 & 40 & 1311840 & 2.5 & & 818.7 & 852.9 & 818.9 & 818.7 & 819.4 \\
\hline 6 & 40 & 2629440 & 5.0 & 823 & 818.7 & 853.0 & 819.0 & 818.7 & 819.6 \\
\hline 6 & 40 & 5260320 & 10.0 & & 818.7 & 853.1 & 819.0 & 818.7 & 819.9 \\
\hline 6 & 40 & 10519200 & 20.0 & & 818.7 & 853.2 & 819.1 & 818.7 & 820.2 \\
\hline 6 & 40 & 21038400 & 40.0 & & 818.7 & 853.3 & 819.3 & 818.7 & 820.7 \\
\hline 6 & 65 & 328320 & 0.625 & & 818.7 & 853.5 & 819.4 & 818.7 & 821.1 \\
\hline 6 & 65 & 656640 & 1.25 & & 818.7 & 853.7 & 819.7 & 818.7 & 821.7 \\
\hline 6 & 65 & 1311840 & 2.5 & & 818.7 & 854.1 & 819.9 & 818.7 & 822.5 \\
\hline 6 & 65 & 2629440 & 5.0 & 829 & 818.7 & 854.5 & 820.3 & 818.7 & 823.6 \\
\hline 6 & 65 & 5260320 & 10.0 & & 818.7 & 855.0 & 820.8 & 818.7 & 824.9 \\
\hline 6 & 65 & 10519200 & 20.0 & & 818.7 & 855.8 & 821.4 & 818.7 & 826.6 \\
\hline 6 & 65 & 21038400 & 40.0 & & 818.7 & 856.8 & 822.2 & 818.7 & 828.8 \\
\hline 6 & 90 & 328320 & 0.625 & & 818.7 & 856.8 & 822.3 & 818.7 & 828.2 \\
\hline 6 & 90 & 656640 & 1.25 & & 818.7 & 858.1 & 823.3 & 818.7 & 830.8 \\
\hline 6 & 90 & 1311840 & 2.5 & & 818.7 & 859.9 & 824.6 & 818.7 & 834.0 \\
\hline 6 & 90 & 2629440 & 5.0 & 829 & 818.7 & 862.3 & 826.3 & 818.7 & 838.1 \\
\hline 6 & 90 & 5260320 & 10.0 & & 818.7 & 865.6 & 828.5 & 818.7 & 843.2 \\
\hline 6 & 90 & 10519200 & 20.0 & & 818.7 & 870.0 & 831.3 & 818.7 & 849.7 \\
\hline 6 & 90 & 21038400 & 40.0 & & 818.7 & 875.9 & 834.9 & 818.7 & 857.8 \\
\hline & & & & & & & & & \\
\hline 5.6 & 100 & 318000 & 0.6 & NP & & & 798.6 & 792.0 & 808.5 \\
\hline 5.6 & 100 & 1052400 & 2.0 & & 792.0 & 858.7 & 802.2 & 792.0 & 816.6 \\
\hline 5.6 & 100 & 3153600 & 6.0 & & 792.0 & 848.9 & 807.2 & 792.0 & 827.5 \\
\hline 5.6 & 200 & 318000 & 0.6 & NP & & & 1023.2 & 971.2 & 1075.2 \\
\hline 5.6 & 200 & 1052400 & 2.0 & NP & & & 1065.8 & 1009.6 & 1122.0 \\
\hline 5.6 & 200 & 3153600 & 6.0 & NP & & & 1099.7 & 1044.1 & 1155.2 \\
\hline 5.6 & 300 & 318000 & 0.6 & NP & & & 1182.7 & 1164.5 & 1200.9 \\
\hline 5.6 & 300 & 1052400 & 2.0 & NP & & & 1188.7 & 1175.1 & 1202.3 \\
\hline 5.6 & 300 & 3153600 & 6.0 & NP & & & 1192.4 & 1182.2 & 1202.6 \\
\hline & & & & & & & & & \\
\hline 7.7 & 100 & 318000 & 0.6 & 759 & 699.0 & 746.7 & 708.3 & 702.2 & 714.4 \\
\hline 7.7 & 100 & 1052400 & 2.0 & & 699.0 & 751.7 & 712.8 & 704.1 & 721.5 \\
\hline 7.7 & 100 & 3153600 & 6.0 & & 699.0 & 746.6 & 718.7 & 706.7 & 730.7 \\
\hline 7.7 & 200 & 318000 & 0.6 & 1103 & 1004.2 & 1111.9 & 1058.1 & 1019.8 & 1096.3 \\
\hline 7.7 & 200 & 1052400 & 2.0 & & 1069.7 & 1183.0 & 1126.3 & 1084.2 & 1168.5 \\
\hline 7.7 & 200 & 3153600 & 6.0 & & 1126.6 & 1241.7 & 1184.1 & 1140.8 & 1227.4 \\
\hline 7.7 & 300 & 318000 & 0.6 & NP & & & 1362.9 & 1348.7 & 1377.1 \\
\hline 7.7 & 300 & 1052400 & 2.0 & NP & & & 1374.6 & 1363.5 & 1385.8 \\
\hline 7.7 & 300 & 3153600 & 6.0 & NP & & & 1382.2 & 1373.4 & 1390.9 \\
\hline
\end{tabular}


Table 4.13. Experimentally Measured and Predicted Mean HV Values for Various U-Nb Alloys for Current and Future Model Validation. The $95 \%$ prediction and confidence intervals are also given. The alloy identified as $6 \mathrm{wt} \% \mathrm{Nb}$ is the RFP banded $\mathrm{U}-6 \mathrm{Nb}$; the alloys identified as $5.6 \mathrm{wt} \%$ and $7.7 \mathrm{wt} \%$ are nonbanded. "NP" indicates that no data point is expected, because of material limitations.

\begin{tabular}{|c|c|c|c|c|c|c|c|c|c|}
\hline \multirow{3}{*}{$\begin{array}{l}\text { Alloy } \\
\text { Wt.\% } \\
\text { Nb }\end{array}$} & \multirow{3}{*}{$\begin{array}{l}\text { Aging } \\
\text { Temp } \\
\text { (C) }\end{array}$} & \multirow{2}{*}{\multicolumn{2}{|c|}{$\begin{array}{l}\text { Aging } \\
\text { Time }\end{array}$}} & \multicolumn{6}{|c|}{ Vickers Microhardness } \\
\hline & & & & \multirow{2}{*}{\begin{tabular}{|l} 
Experimental \\
Mean
\end{tabular}} & \multicolumn{2}{|c|}{ Prediction Int. } & \multirow{2}{*}{\begin{tabular}{|l|} 
Prediction \\
Mean
\end{tabular}} & \multicolumn{2}{|c|}{ Confidence Int. } \\
\hline & & $(\min )$. & (years) & & lower & upper & & lower & upper \\
\hline 6 & 40 & 328320 & 0.625 & & 166.7 & 208.7 & 173.9 & 168.7 & 179.0 \\
\hline 6 & 40 & 656640 & 1.25 & & 166.7 & 210.3 & 175.3 & 169.2 & 181.4 \\
\hline 6 & 40 & 1311840 & 2.5 & & 166.7 & 212.2 & 177.0 & 169.8 & 184.3 \\
\hline 6 & 40 & 2629440 & 5.0 & 171 & 166.7 & 214.6 & 179.1 & 170.5 & 187.7 \\
\hline 6 & 40 & 5260320 & 10.0 & & 166.7 & 217.4 & 181.5 & 171.3 & 191.7 \\
\hline 6 & 40 & 10519200 & 20.0 & & 166.7 & 220.9 & 184.4 & 172.2 & 196.5 \\
\hline 6 & 40 & 21038400 & 40.0 & & 166.7 & 225.1 & 187.8 & 173.4 & 202.2 \\
\hline 6 & 65 & 328320 & 0.625 & & 166.7 & 219.3 & 183.6 & 174.0 & 193.2 \\
\hline 6 & 65 & 656640 & 1.25 & & 166.7 & 223.1 & 186.9 & 175.6 & 198.2 \\
\hline 6 & 65 & 1311840 & 2.5 & & 166.7 & 227.6 & 190.7 & 177.4 & 204.0 \\
\hline 6 & 65 & 2629440 & 5.0 & 182 & 166.7 & 233.1 & 195.3 & 179.6 & 210.9 \\
\hline 6 & 65 & 5260320 & 10.0 & & 166.7 & 239.7 & 200.6 & 182.2 & 219.0 \\
\hline 6 & 65 & 10519200 & 20.0 & & 166.7 & 247.5 & 206.9 & 185.3 & 228.4 \\
\hline 6 & 65 & 21038400 & 40.0 & & 171.5 & 256.8 & 214.1 & 189.0 & 239.3 \\
\hline 6 & 90 & 328320 & 0.625 & & 163.6 & 238.7 & 201.2 & 186.1 & 216.3 \\
\hline 6 & 90 & 656640 & 1.25 & & 168.8 & 246.1 & 207.5 & 189.9 & 225.1 \\
\hline 6 & 90 & 1311840 & 2.5 & & 174.8 & 254.9 & 214.8 & 194.4 & 235.3 \\
\hline 6 & 90 & 2629440 & 5.0 & 201 & 181.6 & 265.2 & 223.4 & 199.6 & 247.1 \\
\hline 6 & 90 & 5260320 & 10.0 & & 189.1 & 277.1 & 233.1 & 205.7 & 260.5 \\
\hline 6 & 90 & 10519200 & 20.0 & & 197.5 & 290.8 & 244.2 & 212.7 & 275.6 \\
\hline 6 & 90 & 21038400 & 40.0 & & 206.9 & 306.3 & 256.6 & 220.8 & 292.4 \\
\hline & & & & & & & & & \\
\hline 5.6 & 100 & 318000 & 0.6 & 189 & 157.5 & 228.6 & 193.1 & 175.1 & 211.0 \\
\hline 5.6 & 100 & 1052400 & 2.0 & & 169.6 & 246.3 & 208.0 & 184.8 & 231.1 \\
\hline 5.6 & 100 & 3153600 & 6.0 & & 183.0 & 267.2 & 225.1 & 196.2 & 253.9 \\
\hline 5.6 & 200 & 318000 & 0.6 & 367 & 325.1 & 404.0 & 364.6 & 339.7 & 389.5 \\
\hline 5.6 & 200 & 1052400 & 2.0 & & 358.3 & 442.8 & 400.5 & 371.5 & 429.6 \\
\hline 5.6 & 200 & 3153600 & 6.0 & & 388.3 & 476.5 & 432.4 & 400.7 & 464.1 \\
\hline 5.6 & 300 & 318000 & 0.6 & 530 & 464.0 & 540.4 & 502.2 & 479.4 & 525.1 \\
\hline 5.6 & 300 & 1052400 & 2.0 & & 487.0 & 562.0 & 524.5 & 502.8 & 546.1 \\
\hline 5.6 & 300 & 3153600 & 6.0 & & 504.6 & 577.7 & 541.1 & 521.3 & 561.0 \\
\hline & & & & & & & & & \\
\hline 7.7 & 100 & 318000 & 0.6 & 191 & 123.9 & 276.4 & 200.2 & 157.8 & 242.5 \\
\hline 7.7 & 100 & 1052400 & 2.0 & & 138.2 & 299.7 & 218.9 & 169.0 & 268.9 \\
\hline 7.7 & 100 & 3153600 & 6.0 & & 152.8 & 324.5 & 238.6 & 180.8 & 296.5 \\
\hline 7.7 & 200 & 318000 & 0.6 & 321 & 256.1 & 405.6 & 330.8 & 291.3 & 370.4 \\
\hline 7.7 & 200 & 1052400 & 2.0 & & 281.4 & 440.1 & 360.7 & 313.0 & 408.4 \\
\hline 7.7 & 200 & 3153600 & 6.0 & & 304.5 & 471.6 & 388.1 & 333.7 & 442.5 \\
\hline 7.7 & 300 & 318000 & 0.6 & 527 & 350.5 & 515.2 & 432.9 & 380.3 & 485.4 \\
\hline 7.7 & 300 & 1052400 & 2.0 & & 375.1 & 542.6 & 458.8 & 404.1 & 513.6 \\
\hline 7.7 & 300 & 3153600 & 6.0 & & 396.4 & 564.4 & 480.4 & 425.4 & 535.5 \\
\hline
\end{tabular}


Table 4.14. Lifetime Predictions for U-5.6Nb.

\begin{tabular}{|c|c|c|c|}
\hline \multirow{3}{*}{$\begin{array}{c}\text { Aging } \\
\text { Temperature } \\
\left({ }^{\circ} \mathrm{C}\right)\end{array}$} & \multicolumn{3}{|c|}{ Lifetime (years) } \\
\hline & \multirow{2}{*}{$\begin{array}{c}\text { Predicted } \\
\text { Value }\end{array}$} & \multicolumn{2}{|c|}{ 95\% Confidence Interval } \\
\hline & & Lower Bound & Upper Bound \\
\hline 20 & $2,117,408$ & 41,783 & $3.2 \times 10^{8}$ \\
\hline 25 & 851,245 & 18,296 & $1.2 \times 10^{8}$ \\
\hline 30 & 352,667 & 8,255 & $4.4 \times 10^{7}$ \\
\hline 35 & 150,344 & 3,832 & $1.7 \times 10^{7}$ \\
\hline 40 & 65,862 & 1,828 & $6.7 \times 10^{6}$ \\
\hline 45 & 29,611 & 895 & $2.7 \times 10^{6}$ \\
\hline 50 & 13,646 & 449 & $1.1 \times 10^{6}$ \\
\hline 55 & 6,439 & 231 & $4.9 \times 10^{5}$ \\
\hline 60 & 3,108 & 121 & $2.1 \times 10^{5}$ \\
\hline
\end{tabular}

Table 4.15. Lifetime Predictions for U-7.7Nb.

\begin{tabular}{|l|r|r|r|}
\hline \multirow{2}{*}{$\begin{array}{c}\text { Aging } \\
\text { Temperature }\end{array}\left({ }^{\circ} \mathrm{C}\right)$} & \multicolumn{3}{|c|}{ Lifetime (years) } \\
\cline { 3 - 4 } & Predicted & \multicolumn{1}{|c|}{ 95\% Confidence Interval } \\
\cline { 3 - 4 } & Value & Lower Bound & Upper Bound \\
\hline 20 & $26,714,967$ & 243,514 & $3.3 \times 10^{10}$ \\
\hline 25 & $8,635,443$ & 89,812 & $9.3 \times 10^{9}$ \\
\hline 30 & $2,897,308$ & 34,194 & $2.7 \times 10^{9}$ \\
\hline 35 & $1,007,151$ & 13,413 & $7.9 \times 10^{8}$ \\
\hline 40 & 362,122 & 5,466 & $2.4 \times 10^{8}$ \\
\hline 45 & 134,454 & 2,298 & $7.7 \times 10^{7}$ \\
\hline 50 & 51,156 & 996 & $2.5 \times 10^{7}$ \\
\hline 55 & 20,293 & 444 & $8.4 \times 10^{6}$ \\
\hline 60 & 8,227 & 203 & $2.9 \times 10^{6}$ \\
\hline
\end{tabular}


Table 4.16. Threshold of Fractional and Absolute Property Changes, and Associated Aging Times at $40^{\circ} \mathrm{C}$ where a Statistically Significant Aging Response is Expected to be Experimentally Observable in Banded U-6Nb. The lower and upper bounds of aging time are prediction intervals.

\begin{tabular}{|c|c|c|c|c|c|c|c|c|}
\hline \multirow[t]{3}{*}{ Property } & \multirow{2}{*}{\multicolumn{2}{|c|}{$\begin{array}{l}\text { Threshold } \\
\text { of change }\end{array}$}} & \multicolumn{6}{|c|}{ Time at $40 \mathrm{C}$ to realize change } \\
\hline & & & \multicolumn{3}{|c|}{ years, except where bolded } & \multicolumn{3}{|c|}{ log(time in minutes) } \\
\hline & $f$ & $\Delta \mathrm{P}$ & $\begin{array}{l}\text { Lower } \\
\text { Bound }\end{array}$ & Mean & $\begin{array}{l}\text { Upper } \\
\text { Bound }\end{array}$ & $\begin{array}{l}\text { Lower } \\
\text { Bound }\end{array}$ & Mean & $\begin{array}{l}\text { Upper } \\
\text { Bound }\end{array}$ \\
\hline TE & 0.1610 & -6.31 & 163 & 3,880 & $1,810,000$ & 7.934 & 9.309 & 11.977 \\
\hline UE & 0.0420 & -1.00 & 20 & 326 & 940,000 & 7.029 & 8.234 & 11.694 \\
\hline $1 Y S$ & 0.0078 & 8.1 & 8 day & 40 day & 50 & 4.035 & 4.765 & 7.416 \\
\hline $1 Y S^{*}$ & 0.0410 & 42.7 & 251 day & 3 & 196 & 5.5584 & 6.2460 & 8.0138 \\
\hline $1 \mathrm{YM}$ & 0.1286 & 8573 & 1 day & 27 day & 41 & 3.229 & 4.594 & 7.337 \\
\hline $2 Y S$ & 0.0171 & 9.3 & 5 & 41 & 49,600 & 6.381 & 7.331 & 10.416 \\
\hline UTS & 0.0160 & 6.1 & 972 & 25,200 & $5,420,000,000$ & 8.708 & 10.122 & 15.455 \\
\hline $\mathrm{HV}$ & 0.0540 & 23.4 & 8 & 60 & 4,240 & 6.606 & 7.502 & 9.348 \\
\hline
\end{tabular}

* Threshold set to reach $200 \mathrm{MPa}$; see Section 4.1.3 for details.

Table 4.17. Threshold of Fractional and Absolute Property Changes, and Associated Aging Times at $40^{\circ} \mathrm{C}$ where a Statistically Significant Aging Response is Expected to be Experimentally Observable in Nonbanded U-5.6Nb. The lower and upper bounds of aging time are prediction intervals.

\begin{tabular}{|c|c|c|c|c|c|c|c|c|}
\hline \multirow[t]{3}{*}{ Property } & \multirow{2}{*}{\multicolumn{2}{|c|}{$\begin{array}{l}\text { Threshold } \\
\text { of change }\end{array}$}} & \multicolumn{6}{|c|}{ Time at $40 \mathrm{C}$ to realize change } \\
\hline & & & \multicolumn{3}{|c|}{ years, except where bolded } & \multicolumn{3}{|c|}{ log(time in minutes) } \\
\hline & $f$ & $\Delta \mathrm{P}$ & $\begin{array}{l}\text { Lower } \\
\text { Bound }\end{array}$ & Mean & $\begin{array}{l}\text { Upper } \\
\text { Bound }\end{array}$ & $\begin{array}{l}\text { Lower } \\
\text { Bound }\end{array}$ & Mean & $\begin{array}{l}\text { Upper } \\
\text { Bound }\end{array}$ \\
\hline TE & 0.4182 & -10.84 & 2,310 & 90,100 & $8,160,000$ & 9.085 & 10.675 & 12.632 \\
\hline$\overline{U E}$ & 0.2433 & -5.63 & 1,260 & 24,600 & $2,840,000$ & 8.820 & 10.112 & 12.174 \\
\hline 1YS & 0.0299 & 32.1 & 129 day & 2 & 143 & 5.268 & 5.961 & 7.876 \\
\hline $1 \mathrm{YM}$ & 0.2231 & 15062 & 9 day & 199 day & 120 & 4.093 & 5.457 & 7.801 \\
\hline $2 Y S$ & 0.1310 & 85.8 & 1,910 & 17,000 & $1,180,000$ & 9.003 & 9.950 & 11.792 \\
\hline UTS & 0.2527 & 103.1 & $2,630,000$ & $89,000,000$ & $29,800,000,000$ & 12.141 & 13.670 & 16.195 \\
\hline $\mathrm{HV}$ & 0.0393 & 17.8 & 2 & 17 & 1,280 & 6.063 & 6.959 & 8.829 \\
\hline
\end{tabular}

Table 4.18. Threshold of Fractional and Absolute Property Changes, and Associated Aging Times at $40^{\circ} \mathrm{C}$ where a Statistically Significant Aging Response is Expected to be Experimentally Observable in Nonbanded U-7.7Nb. The lower and upper bounds of aging time are prediction intervals.

\begin{tabular}{|c|c|c|c|c|c|c|c|c|}
\hline \multirow[t]{3}{*}{ Property } & \multirow{2}{*}{\multicolumn{2}{|c|}{$\begin{array}{l}\text { Threshold } \\
\text { of change }\end{array}$}} & \multicolumn{6}{|c|}{ Time at $40 \mathrm{C}$ to realize change } \\
\hline & & & \multicolumn{3}{|c|}{ years, except where bolded } & \multicolumn{3}{|c|}{ log(time in minutes) } \\
\hline & $f$ & $\Delta \mathrm{P}$ & $\begin{array}{l}\text { Lower } \\
\text { Bound }\end{array}$ & Mean & $\begin{array}{l}\text { Upper } \\
\text { Bound }\end{array}$ & $\begin{array}{l}\text { Lower } \\
\text { Bound }\end{array}$ & Mean & $\begin{array}{l}\text { Upper } \\
\text { Bound }\end{array}$ \\
\hline TE & 0.2628 & -10.25 & 1,890 & 99,200 & $114,000,000$ & 8.997 & 10.717 & 13.779 \\
\hline UE & 0.0687 & -1.95 & 813,000 & $22,100,000$ & $103,000,000,000$ & 11.631 & 13.065 & 16.732 \\
\hline $1 Y S$ & 0.0256 & 33.3 & 1 day & 6 day & 3 & 2.971 & 3.928 & 6.246 \\
\hline 1YM & 0.1476 & 7972 & 38 day & 286 day & 26 & 4.737 & 5.614 & $\overline{7.142}$ \\
\hline $2 Y S$ & 0.0438 & 39.0 & 91 day & 3 & 277 & 5.116 & 6.175 & 8.163 \\
\hline UTS & 0.0418 & 29.3 & 371,000 & $3,100,000$ & $1,060,000,000$ & 11.290 & 12.212 & 14.744 \\
\hline $\mathrm{HV}$ & 0.0149 & 7.2 & $5 \mathrm{~min}$ & $5 \mathrm{hr}$ & 37 & 0.659 & 2.479 & 7.292 \\
\hline
\end{tabular}


Table 4.19. Notional Tensile Property Equivalency Table that Would Result from a Future Systematic Study of the Effects of Tensile Geometry and Machining Damage. Values for the zero artificial age condition are provided from the data made available in this report; such tables could also be generated for several representative artificial ages where the properties differ considerably from the zero age condition.

\begin{tabular}{|c|c|c|c|c|c|c|c|c|}
\hline \multirow[t]{2}{*}{$\begin{array}{l}\text { Material } \\
\text { Pedigree }\end{array}$} & \multirow{2}{*}{$\begin{array}{l}\text { Machining } \\
\text { Damage } \\
\text { Condition }\end{array}$} & \multirow[t]{2}{*}{\begin{tabular}{|l} 
Tensile \\
Geometry
\end{tabular}} & \multicolumn{2}{|l|}{$\begin{array}{l}\text { 1YS } \\
\text { slope }\end{array}$} & \multicolumn{2}{|l|}{\begin{tabular}{|l|}
$1 Y S$ \\
stress
\end{tabular}} & \multicolumn{2}{|l|}{$\begin{array}{l}2 Y S \\
\text { stress }\end{array}$} \\
\hline & & & Value & StDev & Value & StDev & Value & StDev \\
\hline Banded RFP U-6Nb & MA & Fig. 2.1 & & & & & & \\
\hline Banded RFP U-6Nb & AM & Fig. 2.1 & & & & & & \\
\hline Banded RFP U-6Nb & MA & Fig. 2.2 & & & & & & \\
\hline Banded RFP U-6Nb & AM & Fig. 2.2 & 53333 & 5774 & 157.3 & 4.7 & 655.0 & 4.4 \\
\hline Banded RFP U-6Nb & MA & Alternate \#1 & & & & & & \\
\hline Banded RFP U-6Nb & AM & Alternate \#1 & & & & & & \\
\hline Nonbanded U-5.6 Nb & MA & Fig. 2.1 & 63800 & 17254 & 125.4 & 16.4 & 545.2 & 46.8 \\
\hline Nonbanded U-5.6Nb & AM & Fig. 2.1 & & & & & & \\
\hline Nonbanded U-5.6Nb & MA & Fig. 2.2 & & & & & & \\
\hline Nonbanded U-5.6Nb & AM & Fig. 2.2 & & & & & & \\
\hline Nonbanded U-5.6Nb & MA & Alternate \#1 & & & & & & \\
\hline Nonbanded U-5.6Nb & AM & Alternate \#1 & & & & & & \\
\hline
\end{tabular}

\begin{tabular}{|c|c|c|c|c|c|c|c|c|}
\hline \multirow{3}{*}{$\begin{array}{l}\text { Material } \\
\text { Pedigree }\end{array}$} & \multirow{3}{*}{$\begin{array}{l}\text { Machining } \\
\text { Damage } \\
\text { Condition }\end{array}$} & \multirow{3}{*}{$\begin{array}{l}\text { Tensile } \\
\text { Geometry }\end{array}$} & \multirow{2}{*}{\multicolumn{2}{|c|}{\begin{tabular}{|l} 
UTS \\
stress
\end{tabular}}} & \multicolumn{4}{|c|}{ Plastic Elongation } \\
\hline & & & & & \multicolumn{2}{|c|}{ Uniform } & \multicolumn{2}{|l|}{ Total } \\
\hline & & & Value & StDev & Value & StDev & Value & StDev \\
\hline Banded RFP U-6Nb & MA & Fig. 2.1 & & & & & & \\
\hline Banded RFP U-6Nb & AM & Fig. 2.1 & & & & & & \\
\hline Banded RFP U-6Nb & MA & Fig. 2.2 & & & & & & \\
\hline Banded RFP U-6Nb & AM & Fig. 2.2 & 818.7 & 2.5 & 0.2382 & 0.0062 & 0.3920 & 0.0424 \\
\hline Banded RFP U-6Nb & MA & Alternate \#1 & & & & & & \\
\hline Banded RFP U-6Nb & AM & Alternate \#1 & & & & & & \\
\hline Nonbanded U-5.6Nb & MA & Fig. 2.1 & 792.0 & 62.1 & 0.2006 & 0.0114 & 0.0111 & 0.0089 \\
\hline Nonbanded U-5.6Nb & AM & Fig. 2.1 & & & & & & \\
\hline Nonbanded U-5.6Nb & MA & Fig. 2.2 & & & & & & \\
\hline Nonbanded U-5.6Nb & AM & Fig. 2.2 & & & & & & \\
\hline Nonbanded U-5.6Nb & MA & Alternate \#1 & & & & & & \\
\hline Nonbanded U-5.6Nb & AM & Alternate \#1 & & & & & & \\
\hline
\end{tabular}




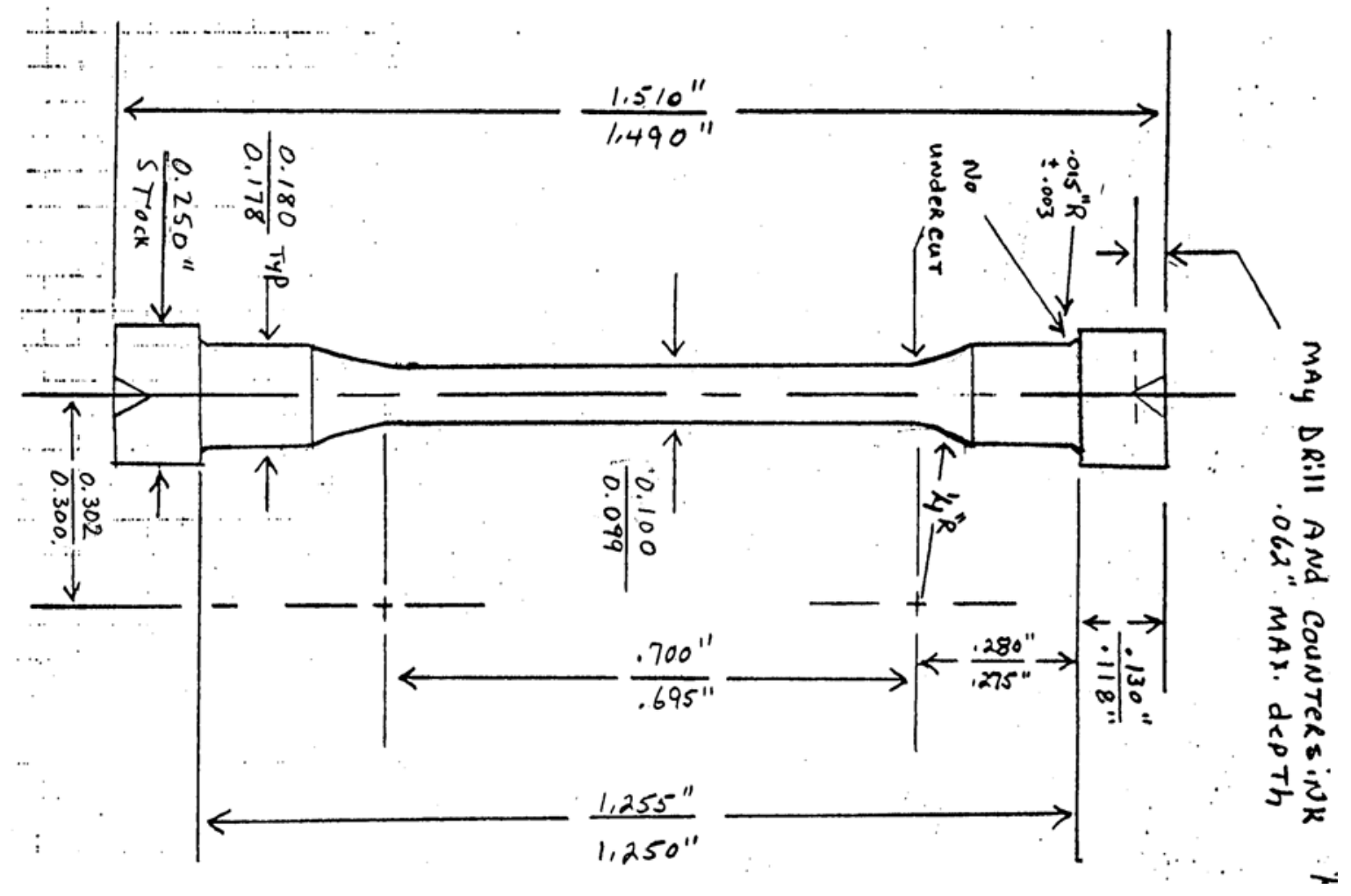

Figure 2.1. Cylindrical tensile specimen geometry used for the nonbanded U-5.6Nb and U-7.7Nb studies for both model fitting and for validation. Dimensions are in inches.

1 inch round button head tensile sample

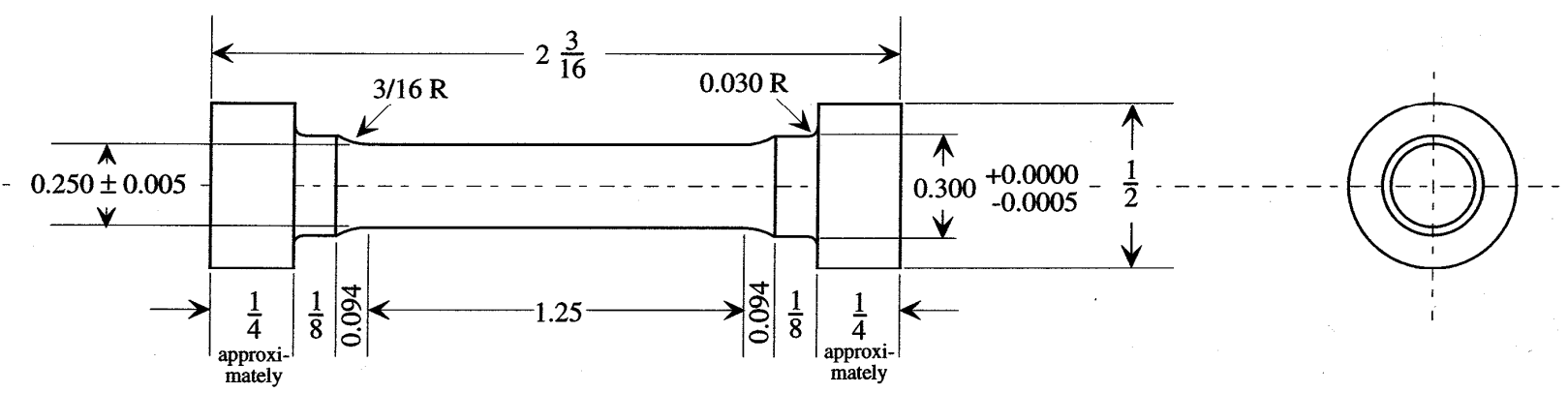

Figure 2.2. Cylindrical tensile specimen geometry used for the banded U-6Nb validation experiments. Dimensions are in inches. 


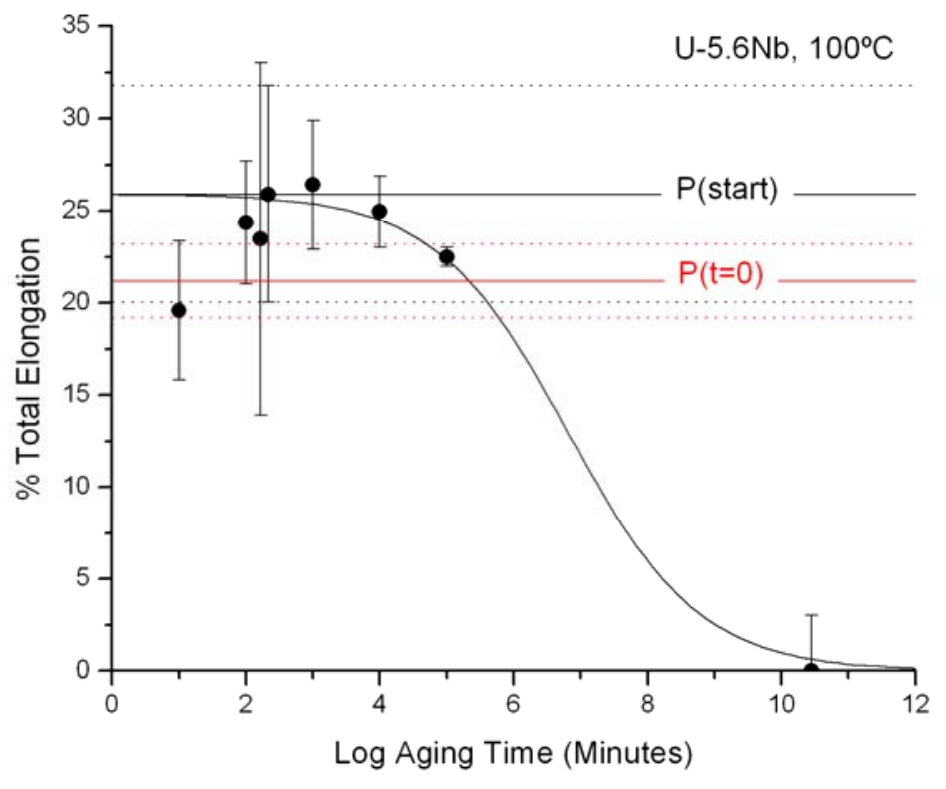

Figure 3.1. Total elongation at $100^{\circ} \mathrm{C}$ for U-5.6Nb. The black and red dotted lines indicate \pm 1 standard deviation about the mean values (solid lines) of $P($ start $)$ and $P(t=0)$, respectively. A fictitious end-of-hardening (peak age) point has been added at $x=10.44$ to guide the Boltzmann fit in the previous study. All other solid points are data, with \pm 1 standard deviation shown.

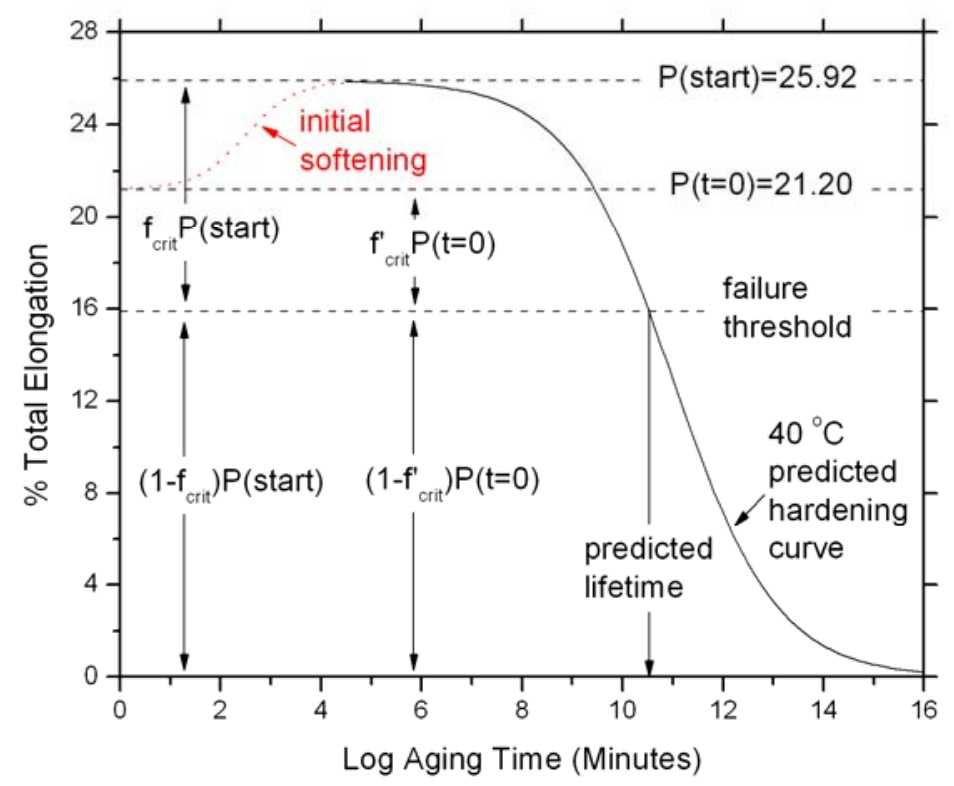

Figure 3.2. Predicted TE (solid curve) and lifetime at $40^{\circ} \mathrm{C}$ for U-5.6Nb (solid curve). The defining relationships between the failure threshold, $P($ start $), P(t=0), f_{\text {crit }}$ and $f_{\text {crit }}^{\prime}$ are graphically depicted. The differentiation between $f_{\text {crit }}$ and $f_{\text {crit }}^{\prime}$ is due to the initial softening transient that is schematically depicted (red dotted curve). See the text for further details. 


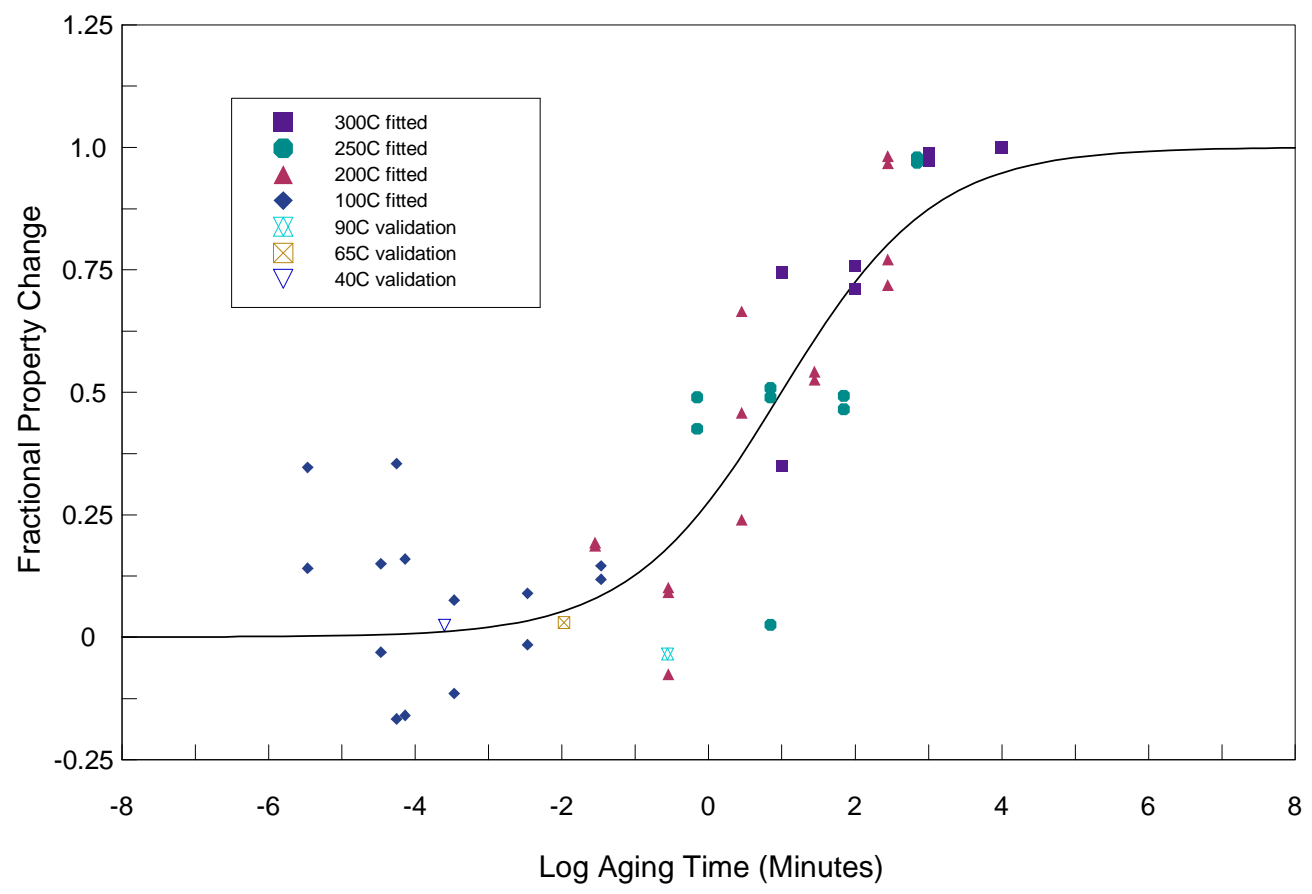

Figure 4.1. Universal plot of U-5.6Nb TE data (points) and model fit to data (solid line).

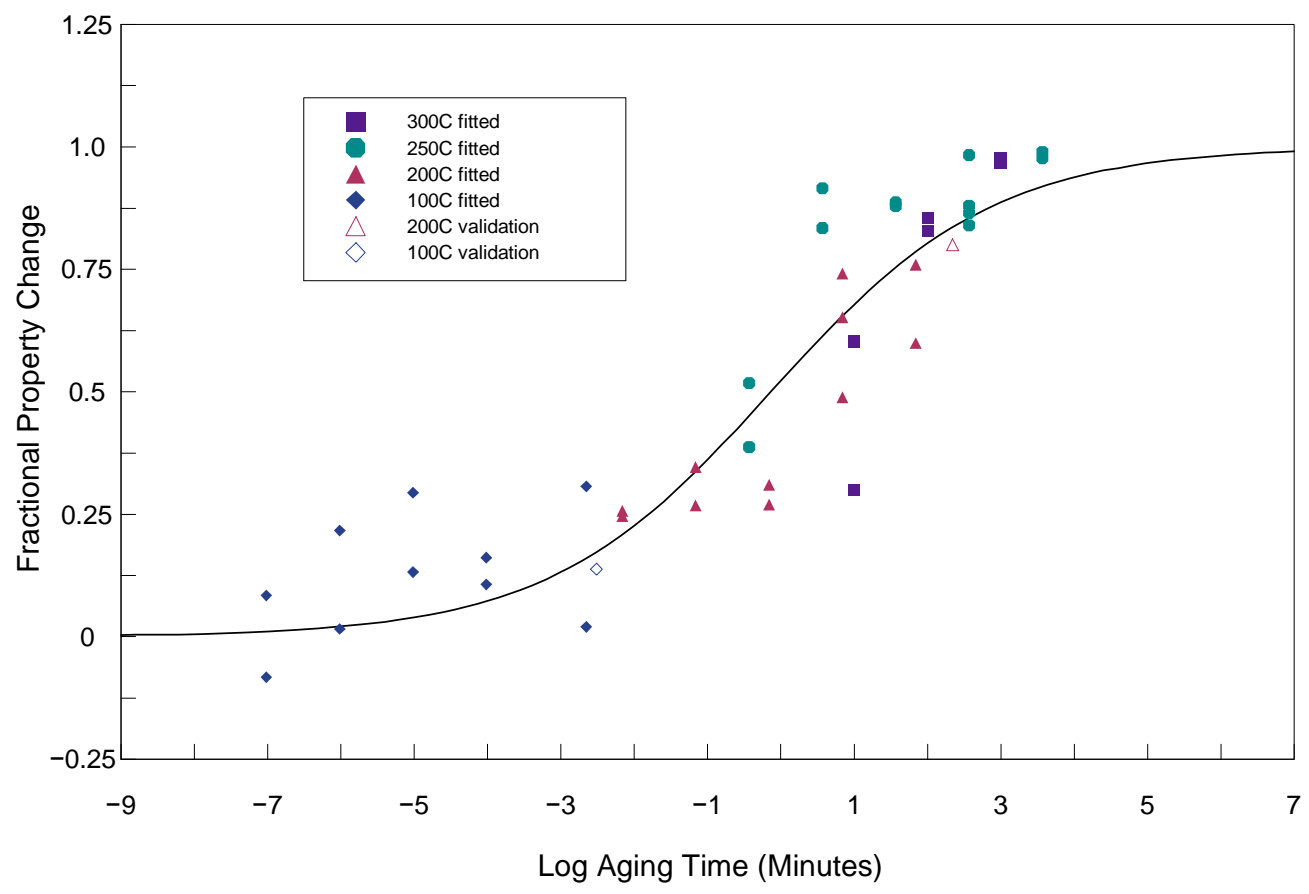

Figure 4.2. Universal plot of U-7.7Nb TE data (points) and model fit to data (solid line). 


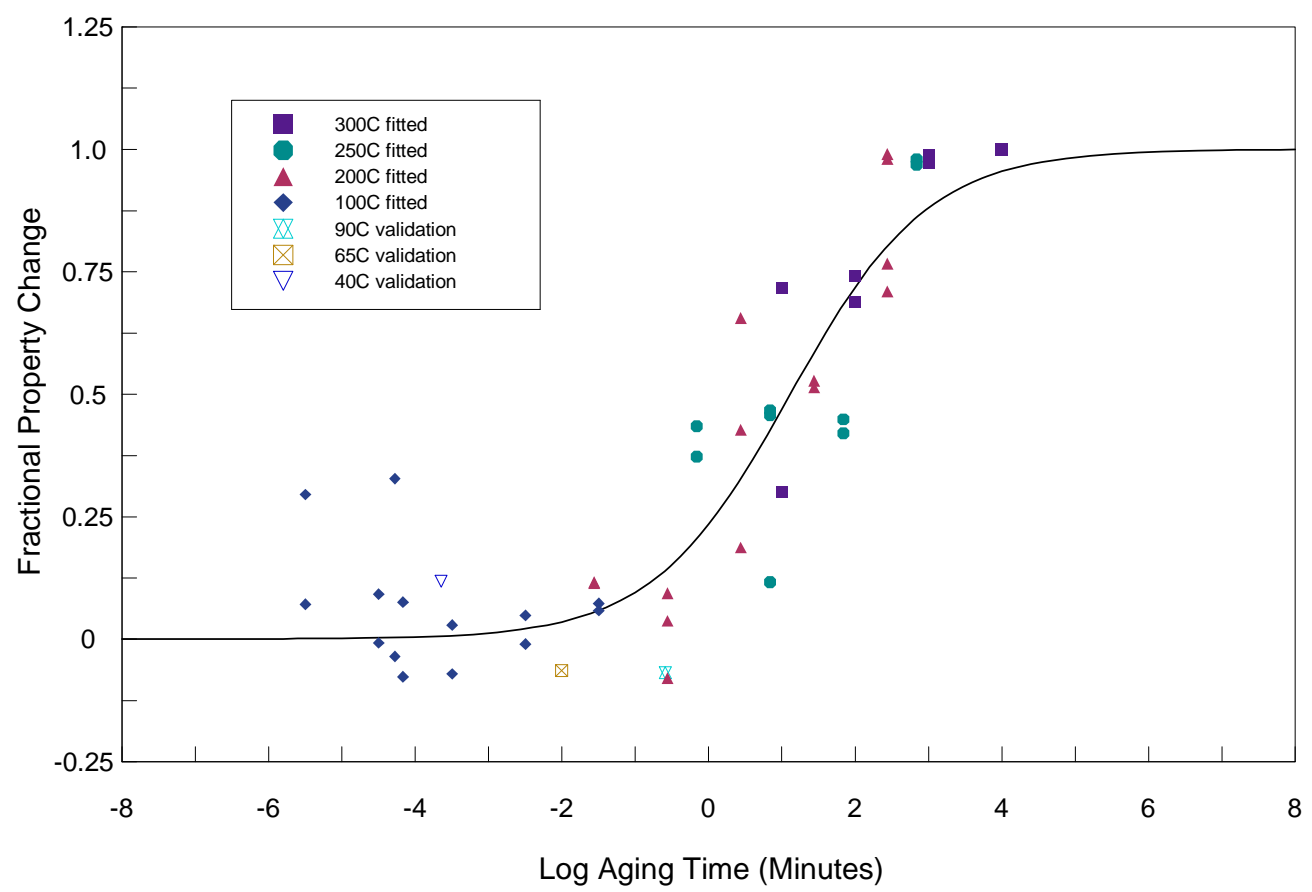

Figure 4.3. Universal plot of U-5.6Nb UE data (points) and model fit to data (solid line).

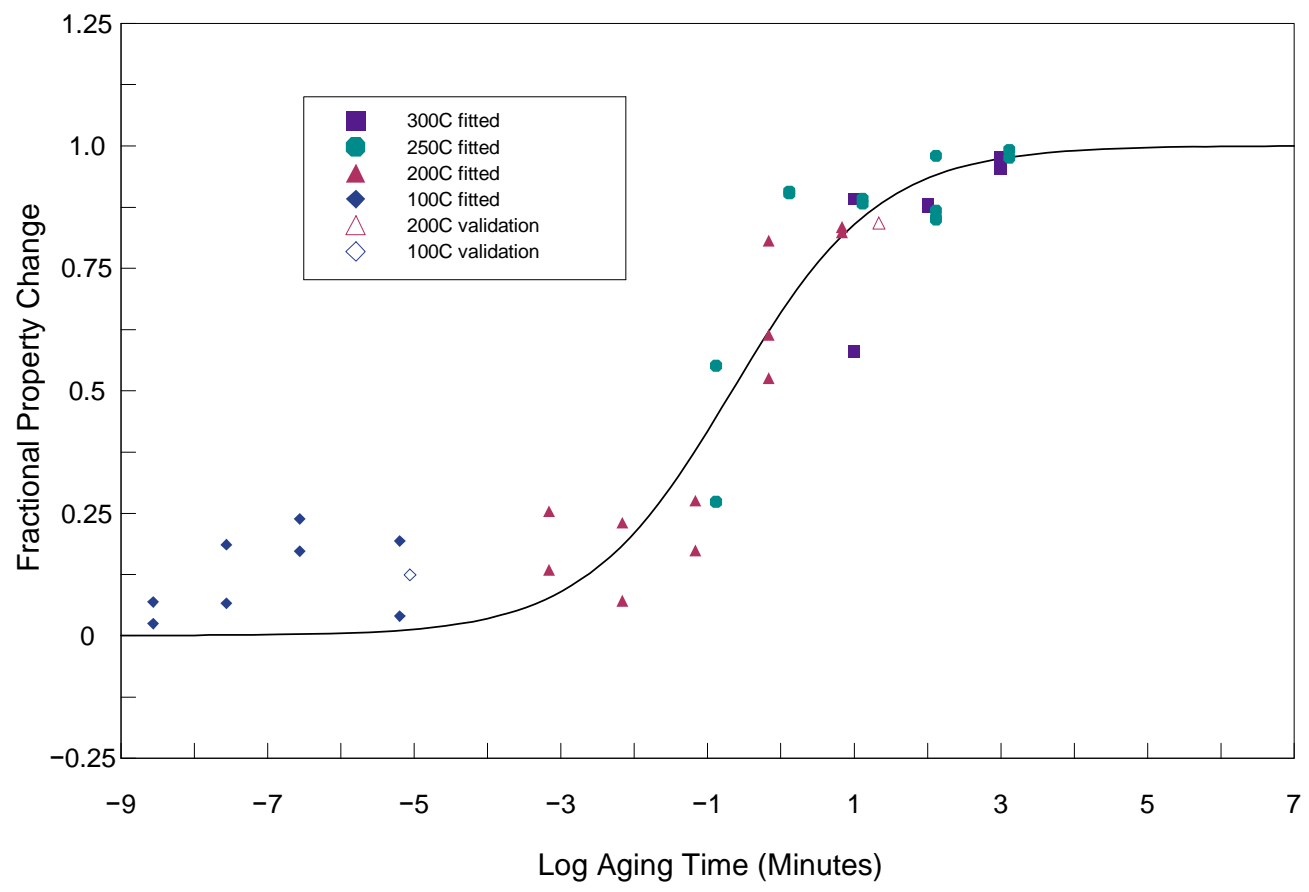

Figure 4.4. Universal plot of U-7.7Nb UE data (points) and model fit to data (solid line). 


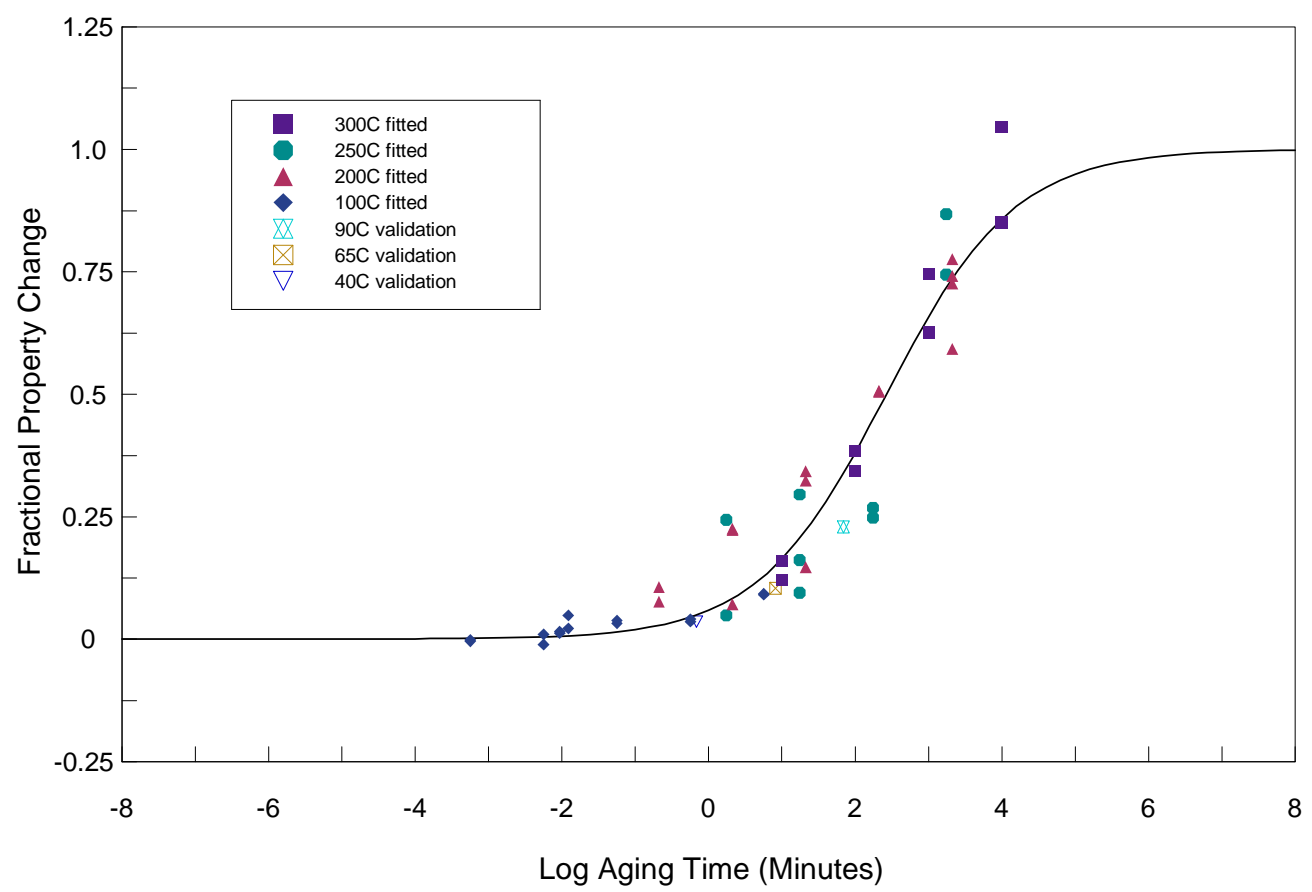

Figure 4.5. Universal plot of U-5.6Nb 1YS data (points) and model fit to data (solid line).

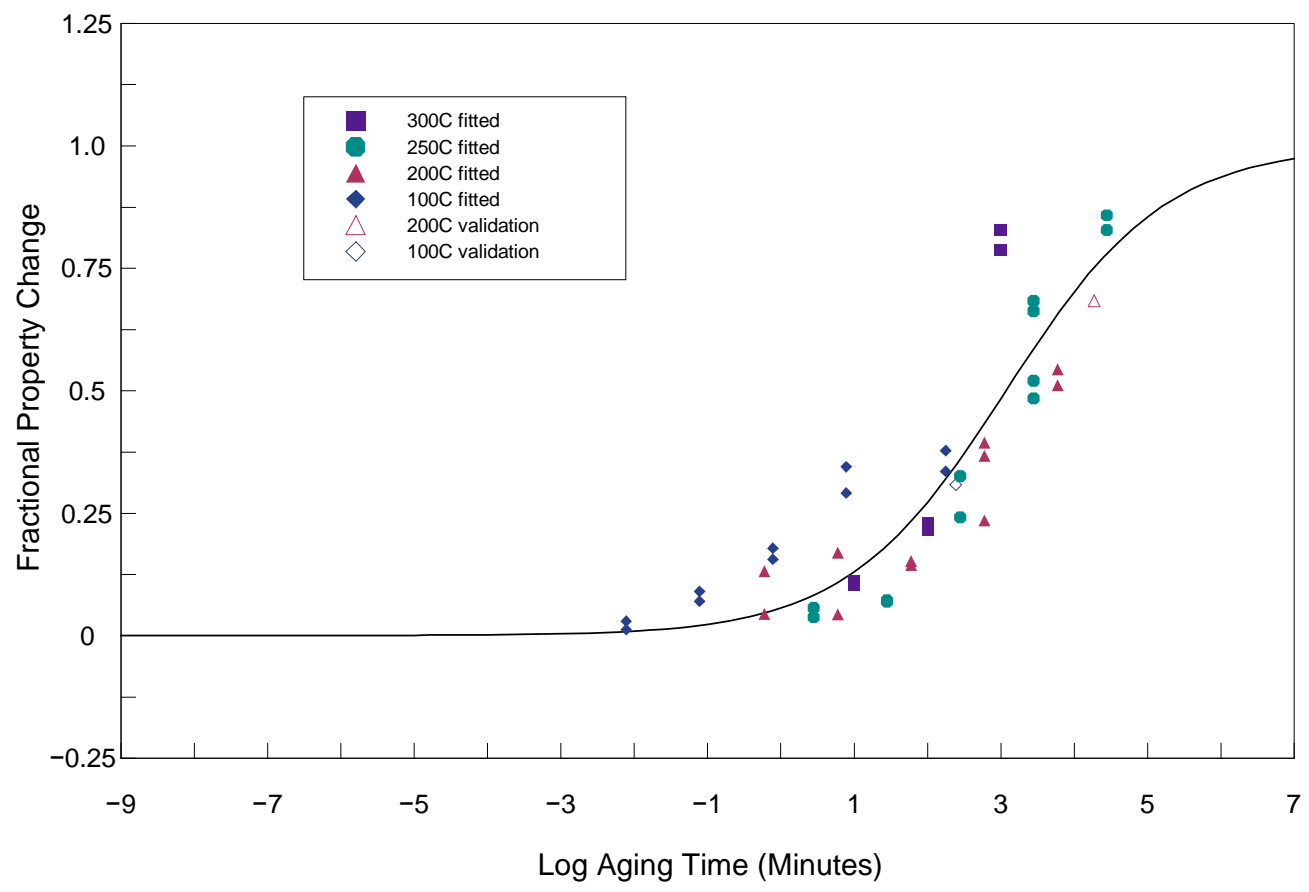

Figure 4.6. Universal plot of U-7.7Nb 1YS data (points) and model fit to data (solid line). 


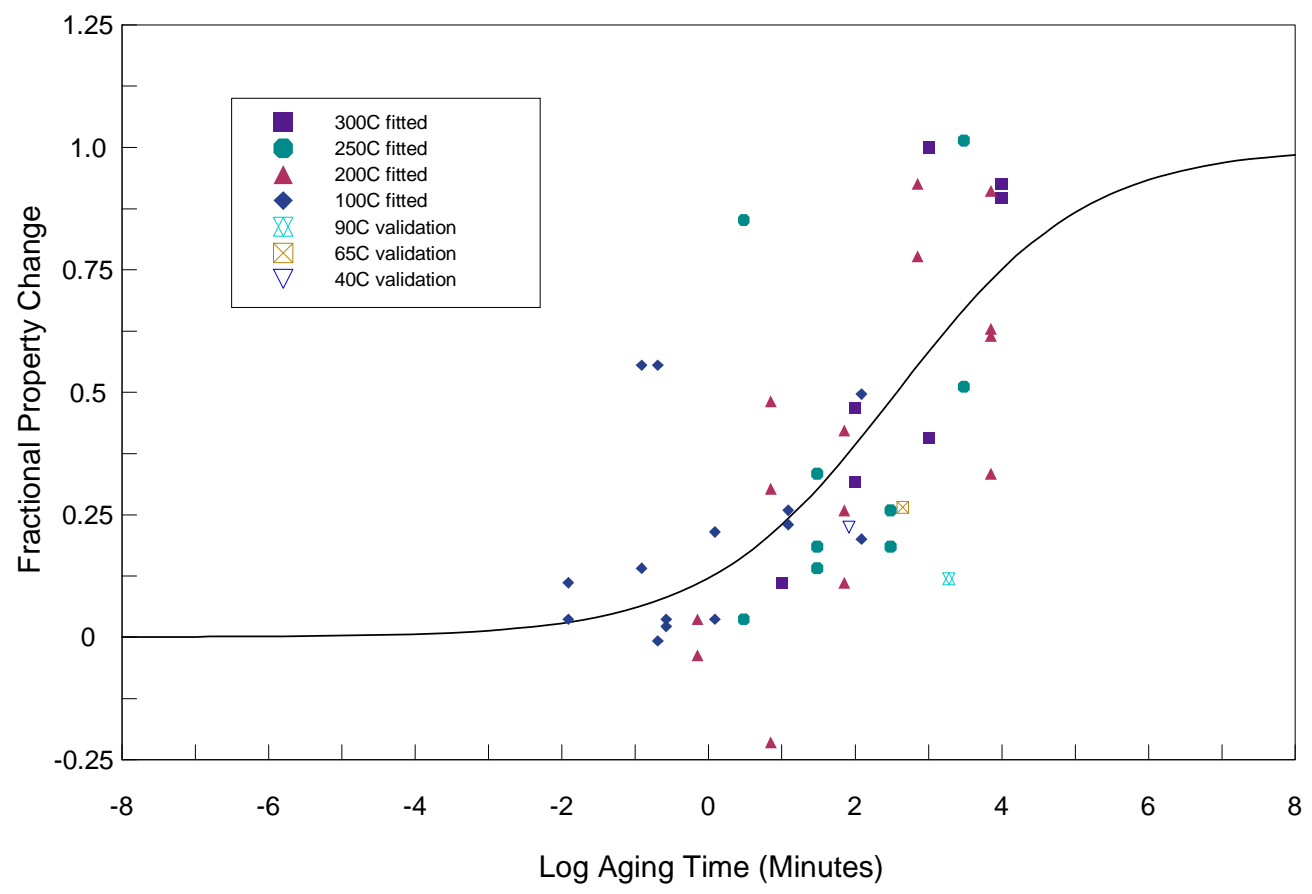

Figure 4.7. Universal plot of U-5.6Nb 1YM data (points) and model fit to data (solid line).

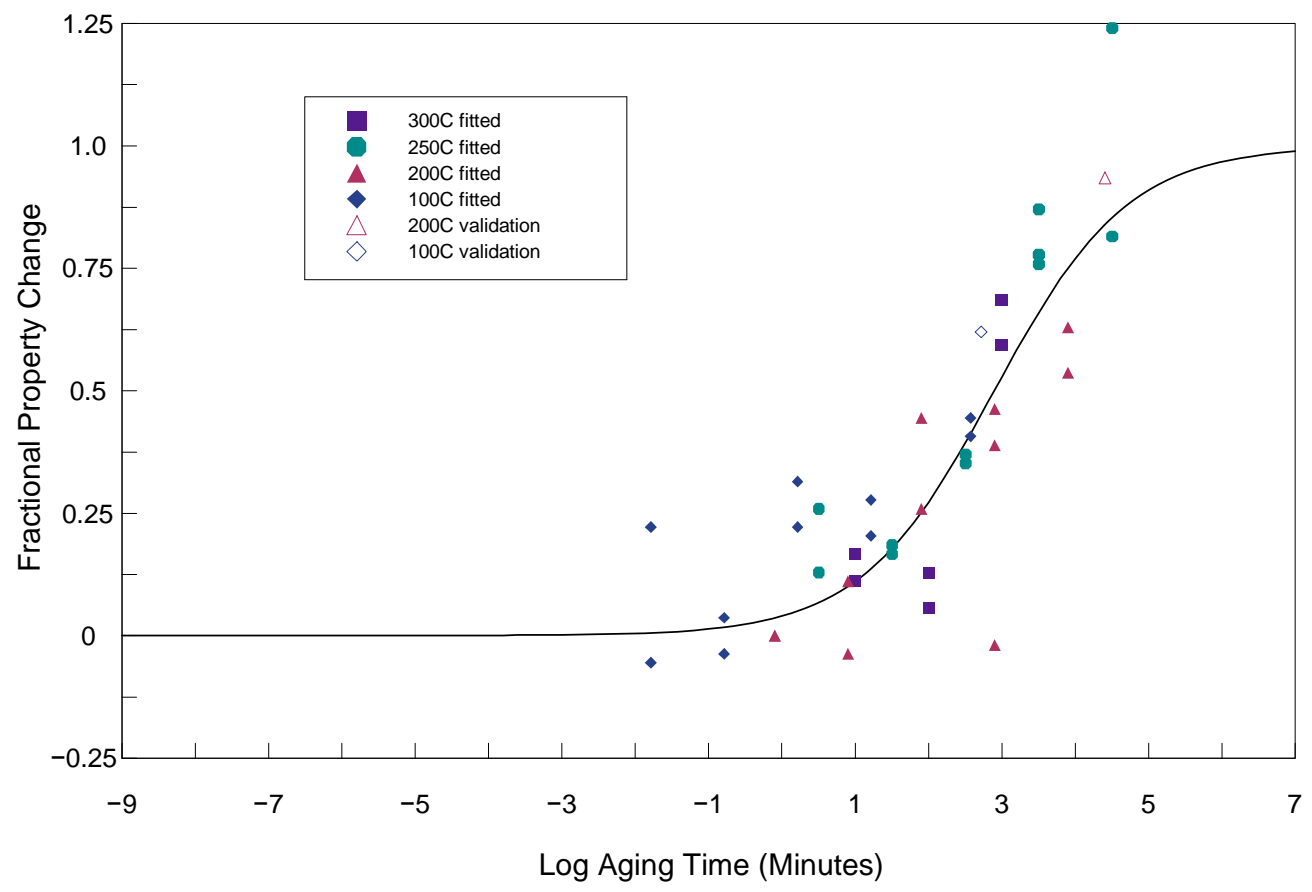

Figure 4.8. Universal plot of U-7.7Nb 1YM data (points) and model fit to data (solid line). 


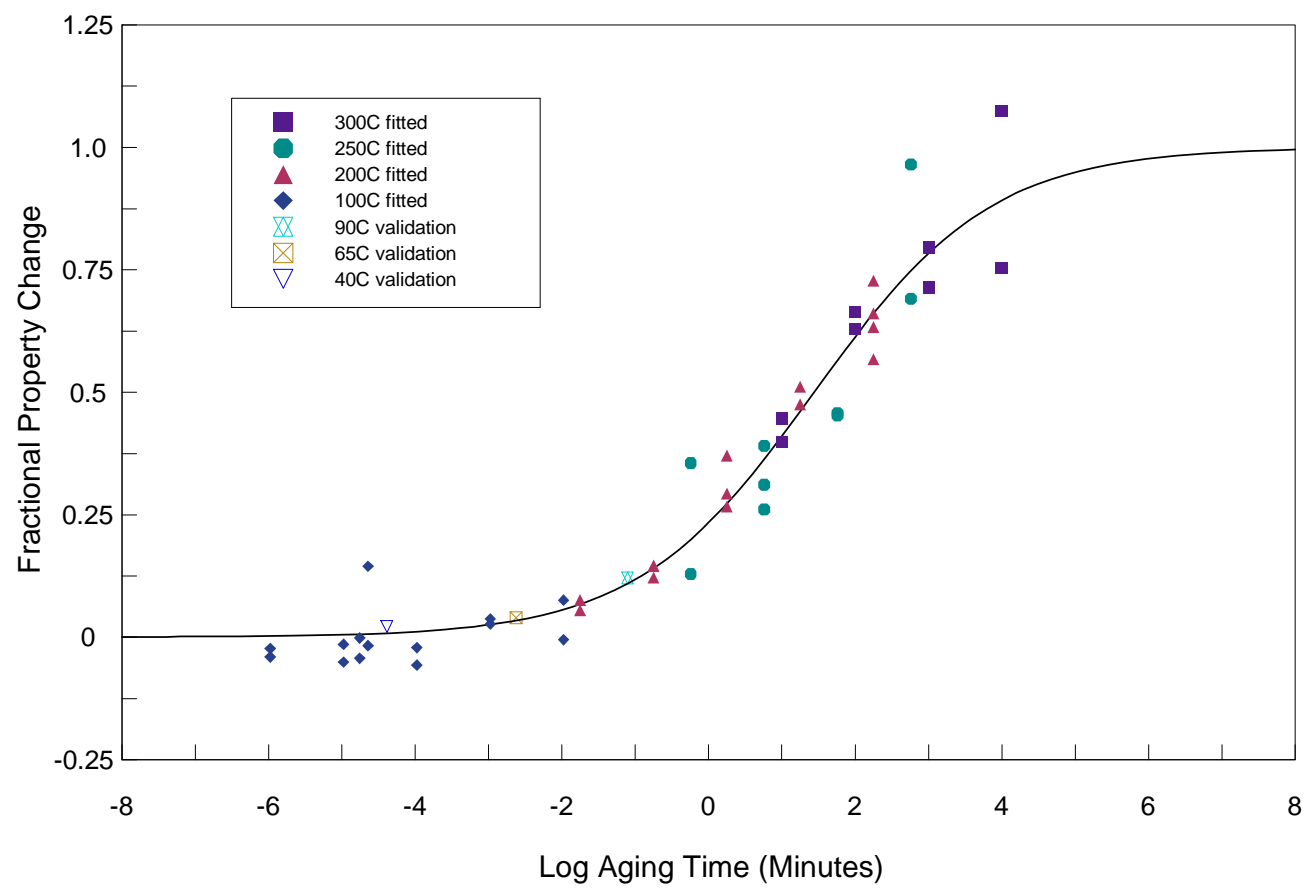

Figure 4.9. Universal plot of U-5.6Nb 2YS data (points) and model fit to data (solid line).

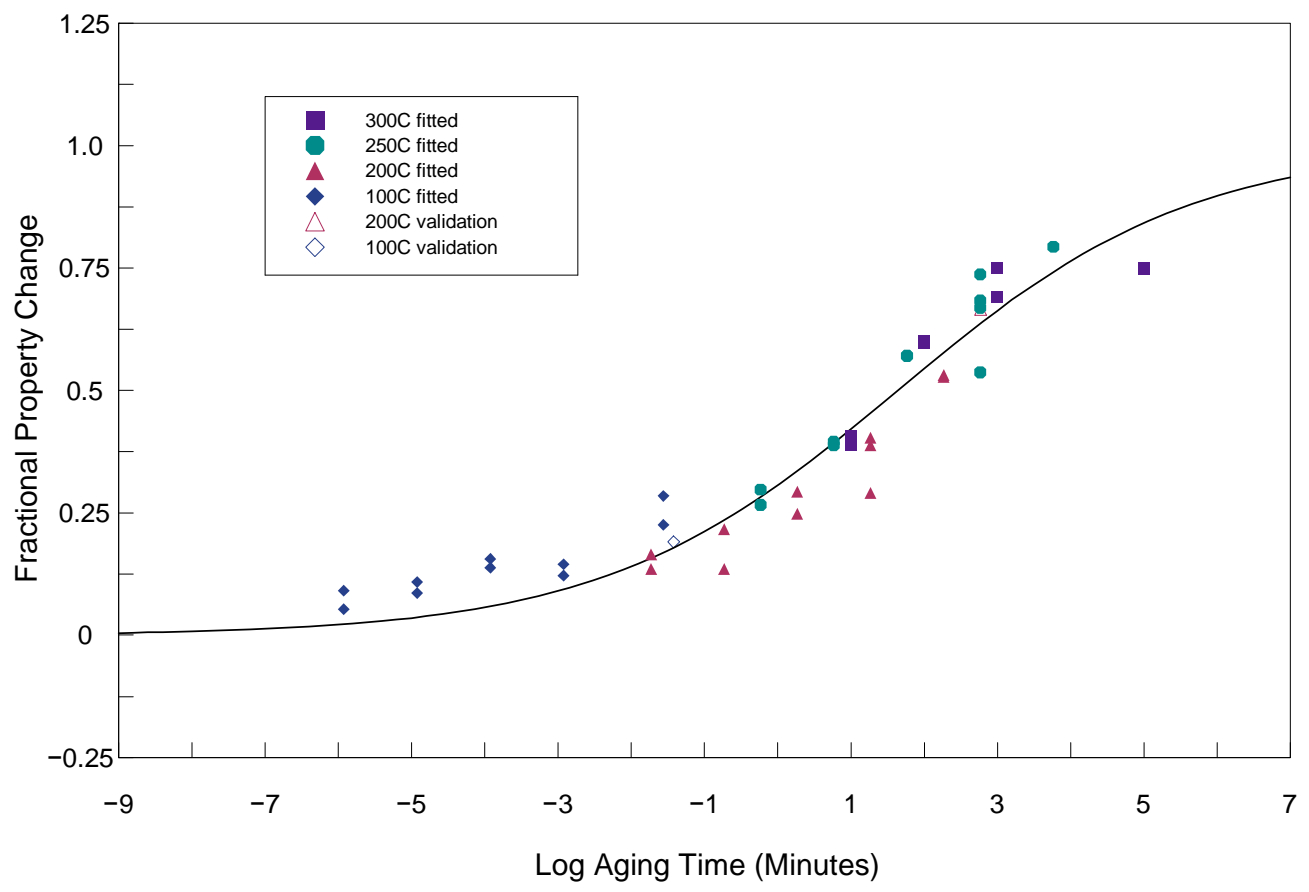

Figure 4.10. Universal plot of U-7.7Nb 2YS data (points) and model fit to data (solid line). 


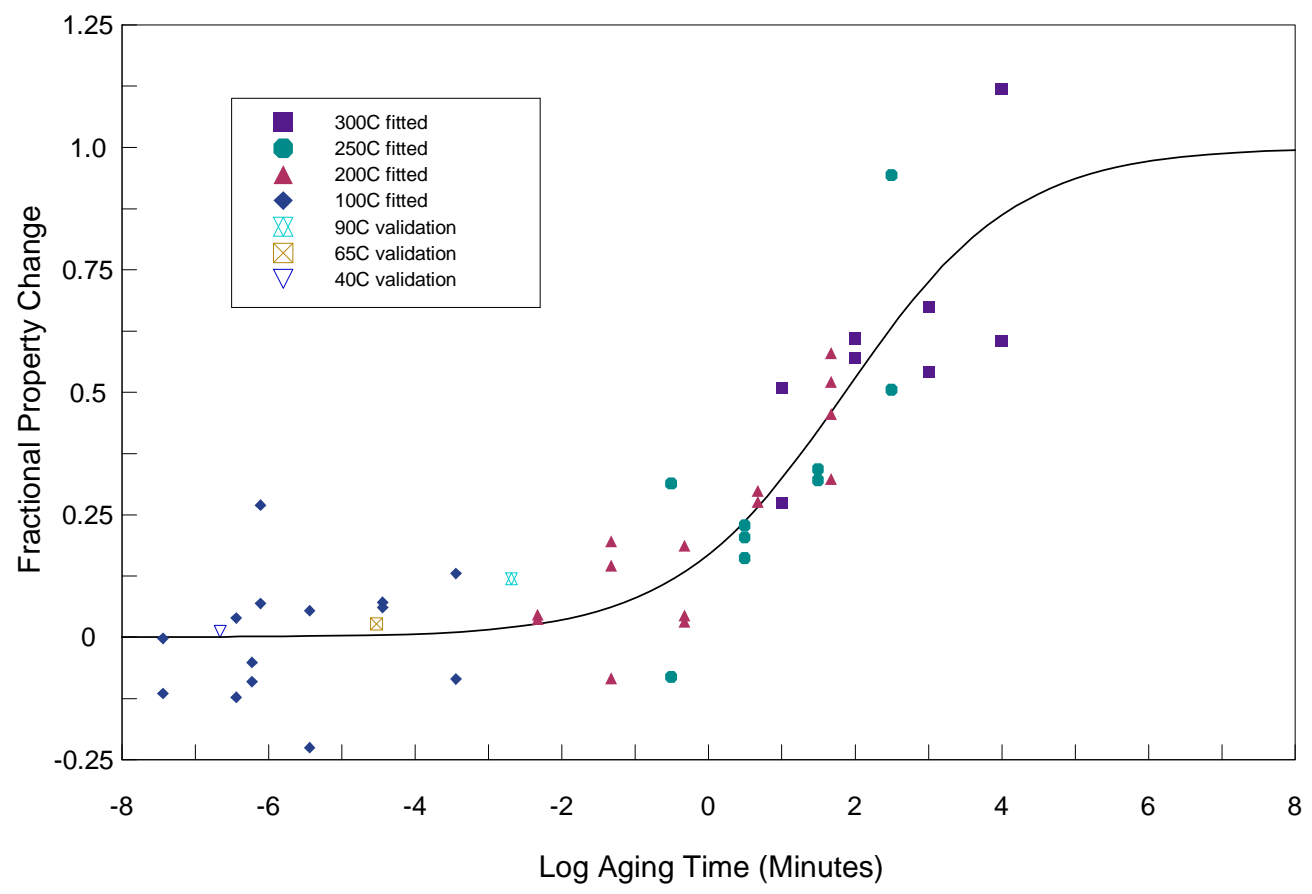

Figure 4.11. Universal plot of U-5.6Nb UTS data (points) and model fit to data (solid line).

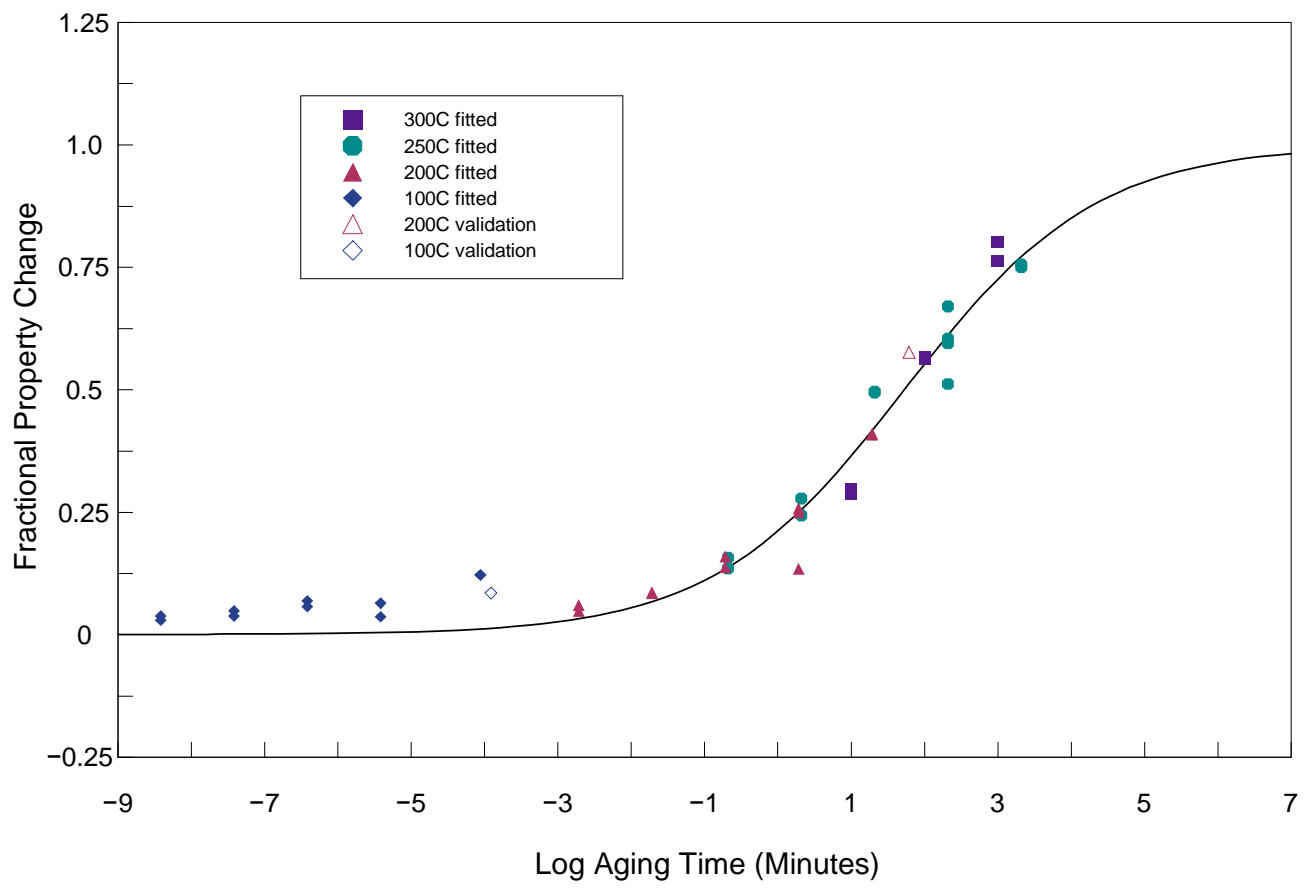

Figure 4.12. Universal plot of U-7.7Nb UTS data (points) and model fit to data (solid line). 


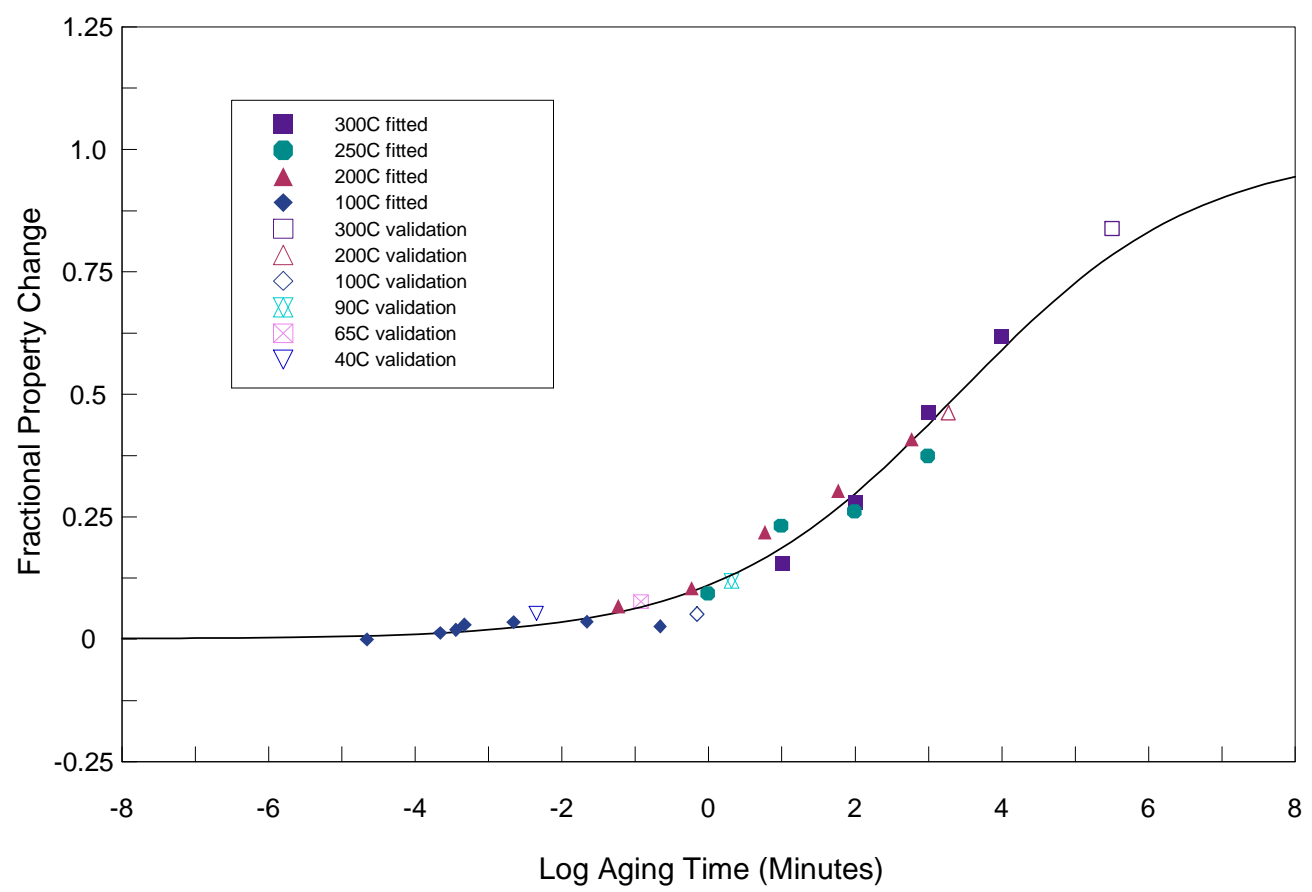

Figure 4.13. Universal plot of U-5.6Nb HV data (points) and model fit to data (solid line).

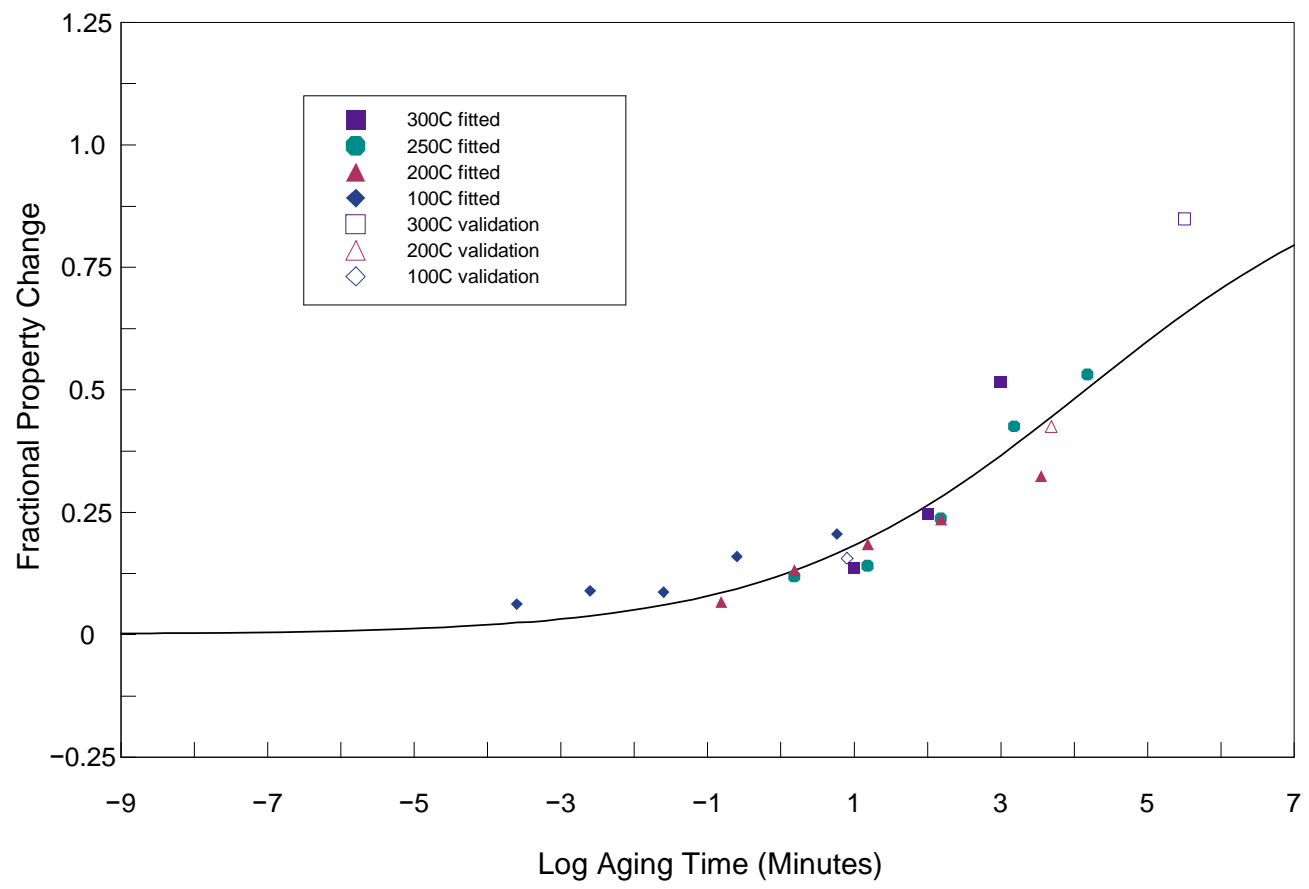

Figure 4.14. Universal plot of U-7.7Nb HV data (points) and model fit to data (solid line). 


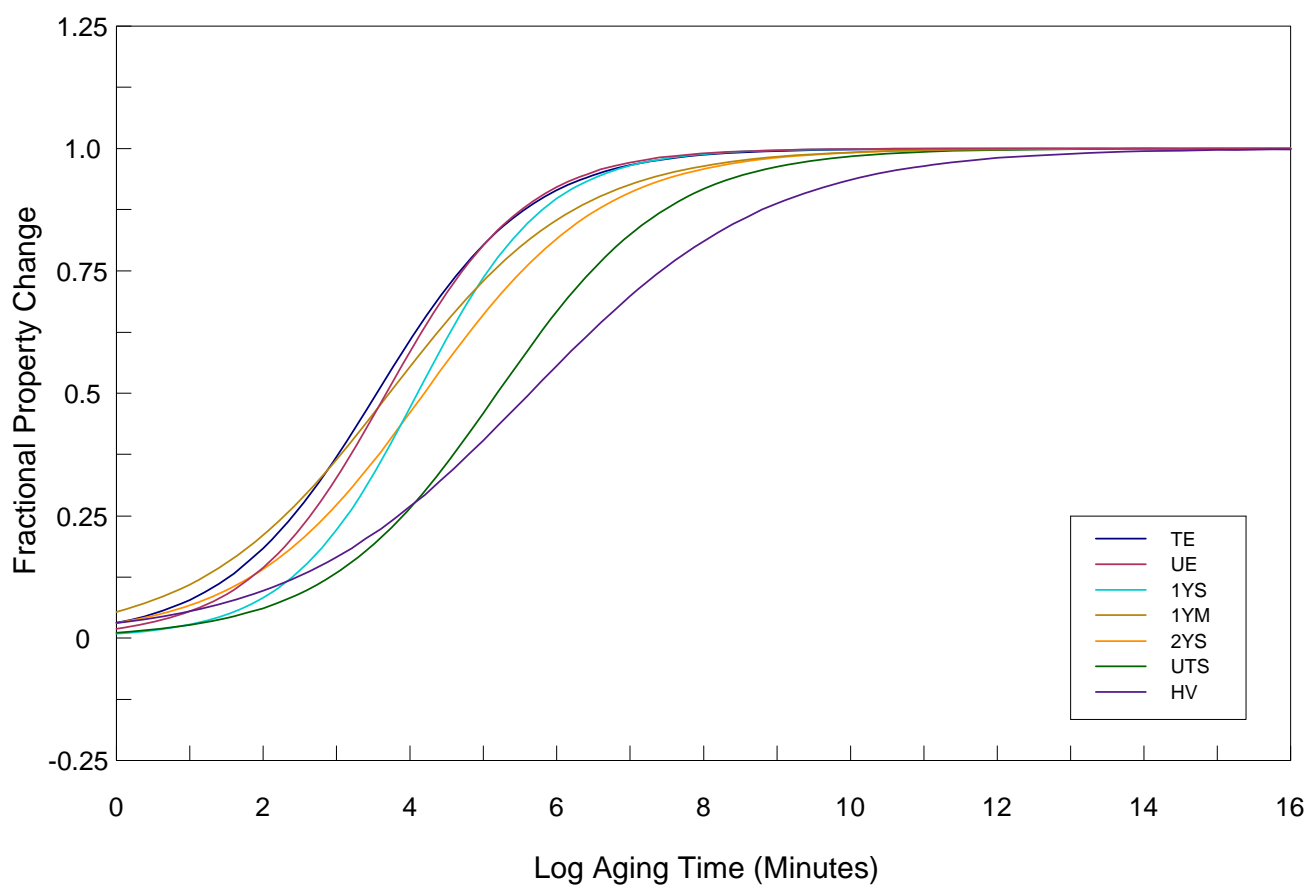

(a)

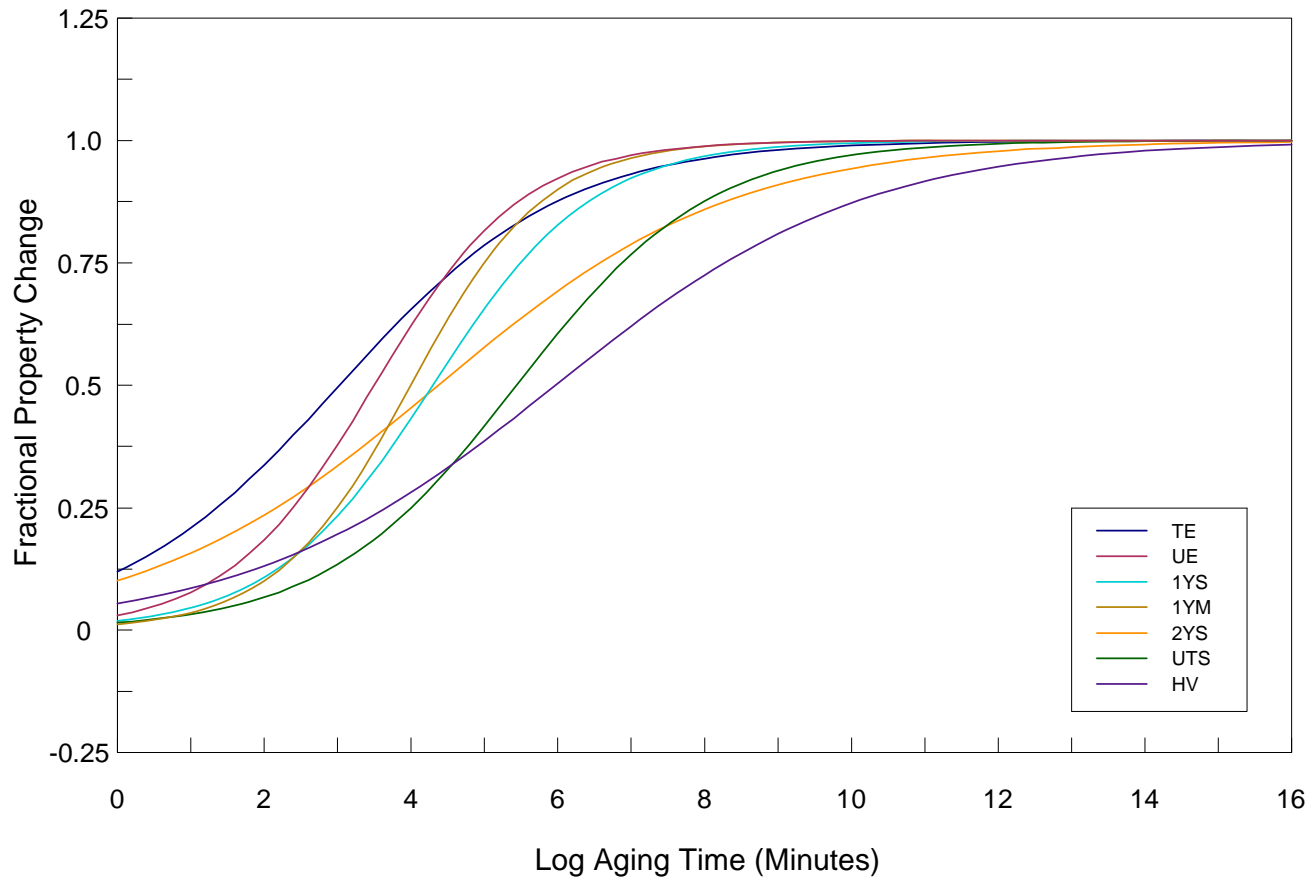

(b)

Figure 4.15. Consolidated aging response model fits at $200^{\circ} \mathrm{C}$ for (a) U-5.6Nb and (b) U-7.7Nb. 


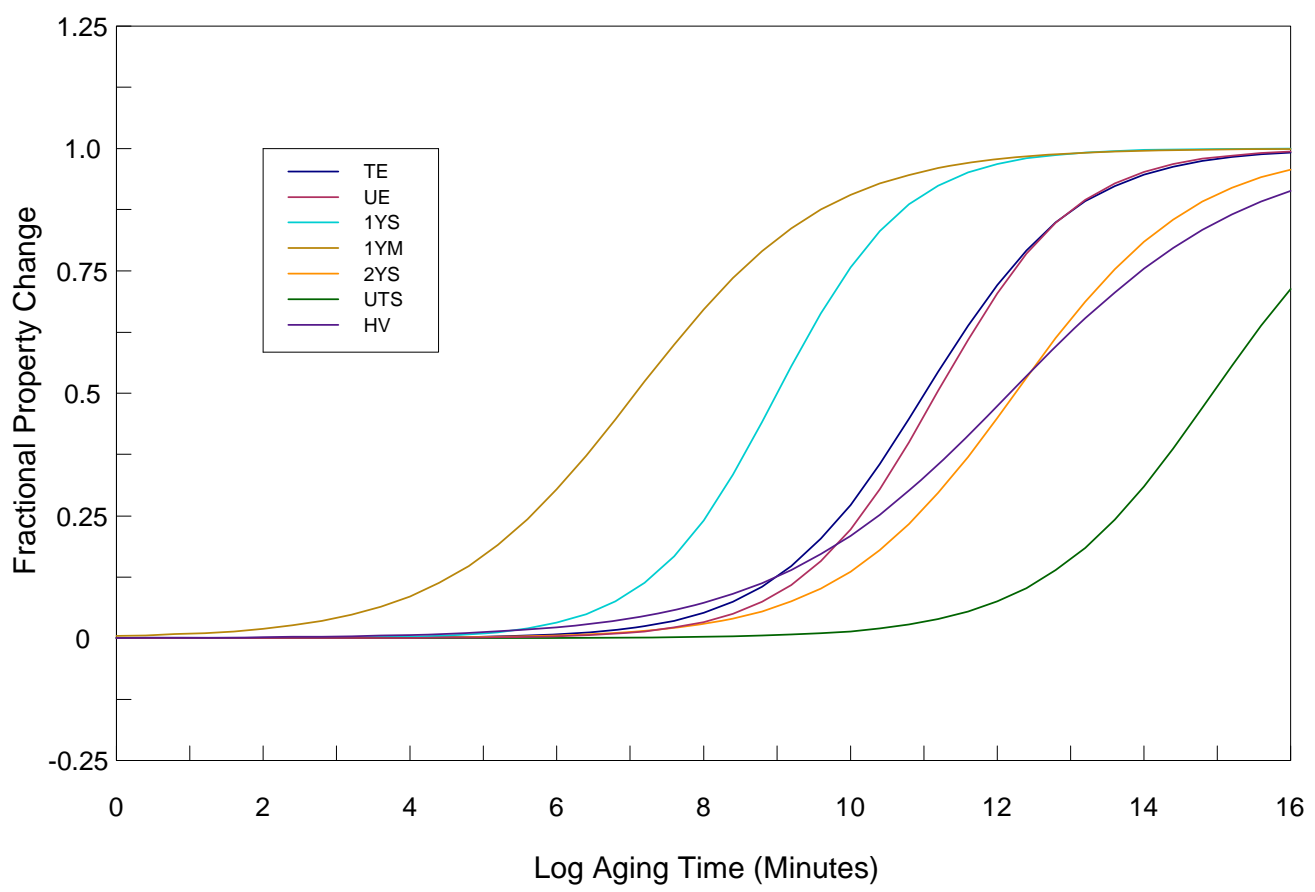

(a)

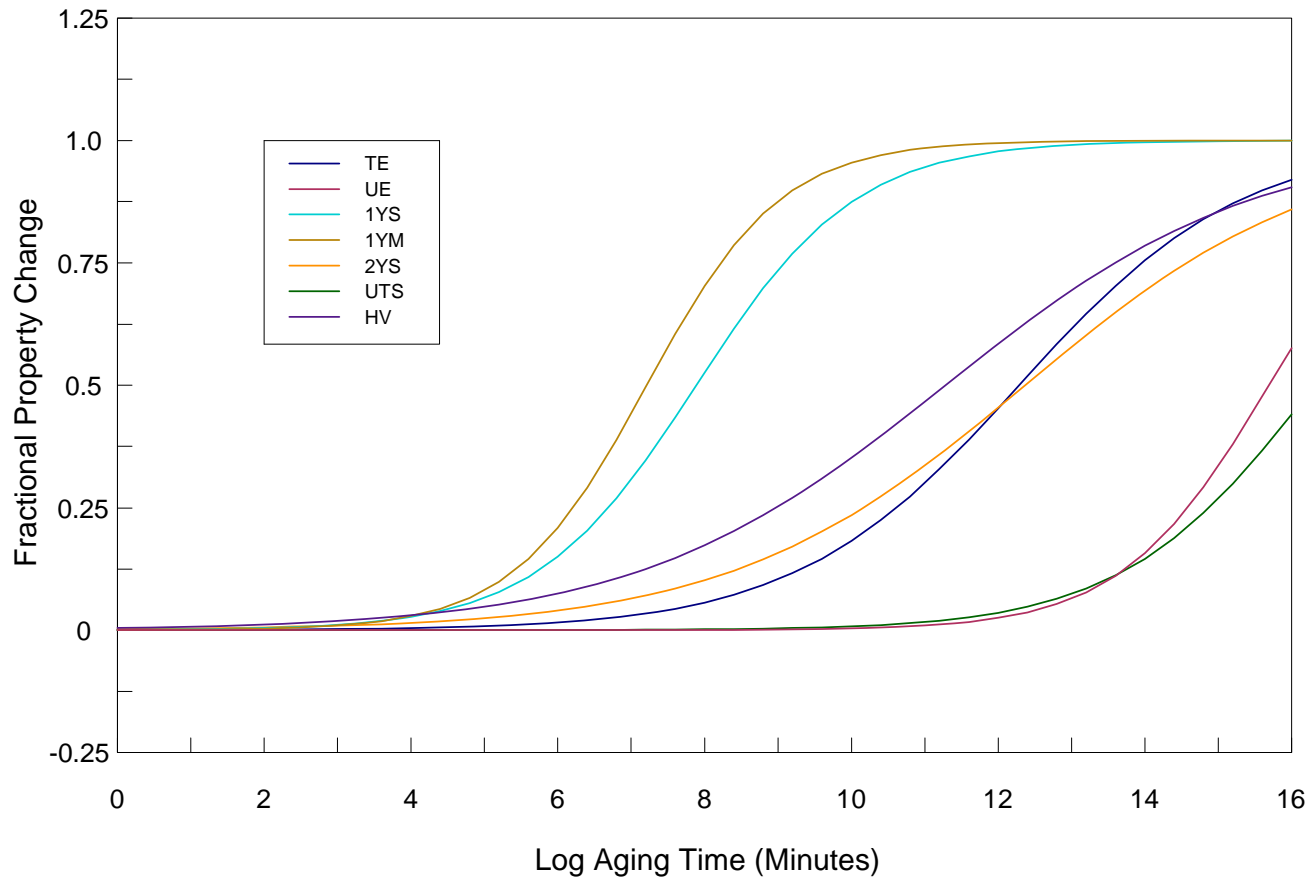

(b)

Figure 4.16. Consolidated aging response predictions at $40^{\circ} \mathrm{C}$ for (a) $\mathrm{U}-5.6 \mathrm{Nb}$ and (b) U-7.7Nb. 


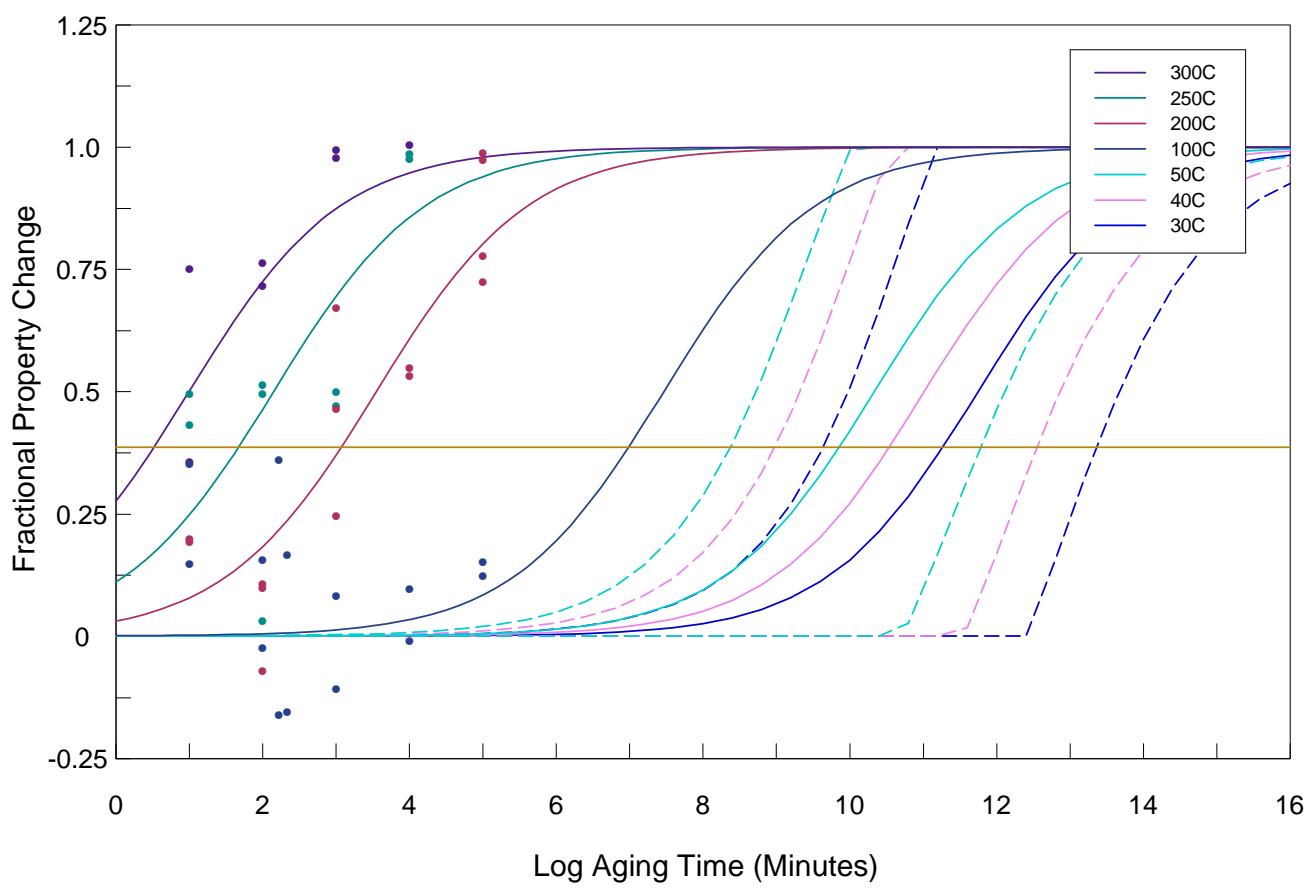

(a)

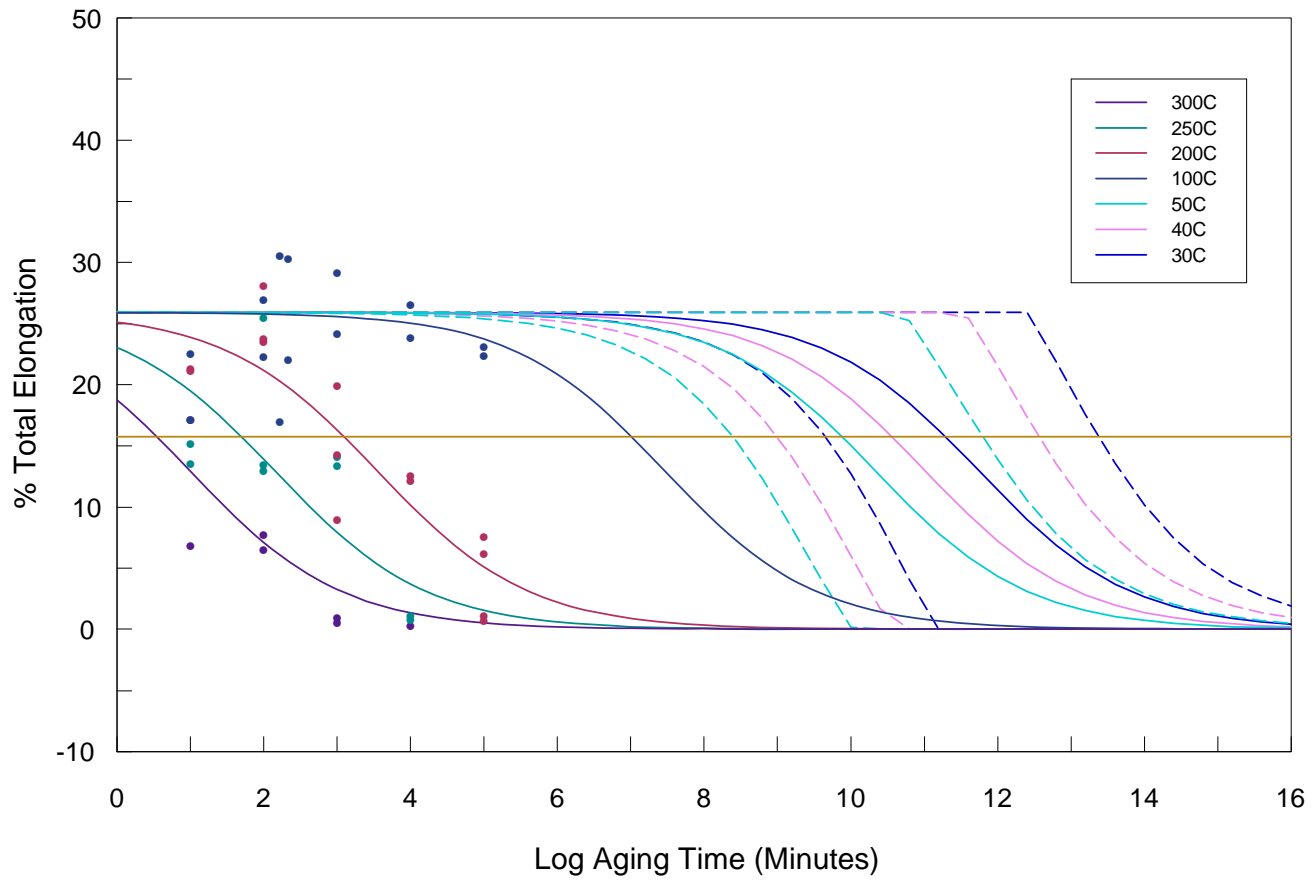

(b)

Figure 4.17. U-5.6Nb TE data (points), model fits to data (solid lines), and low-temperature model predictions (solid line-mean, dashed lines_-95\% confidence intervals). (a) in terms of $f$, (b) in terms of absolute property. The yellow line is the failure threshold. 


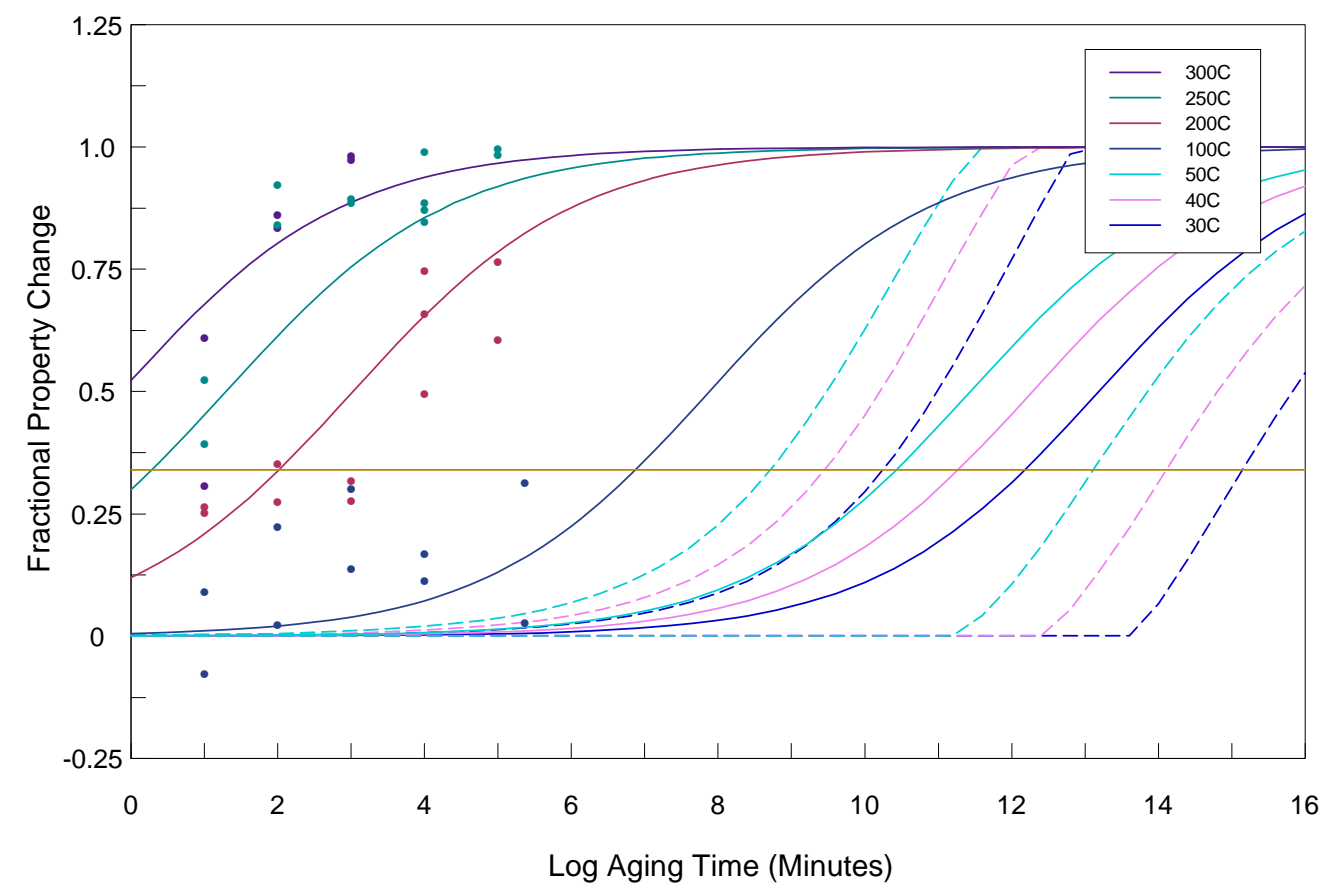

(a)

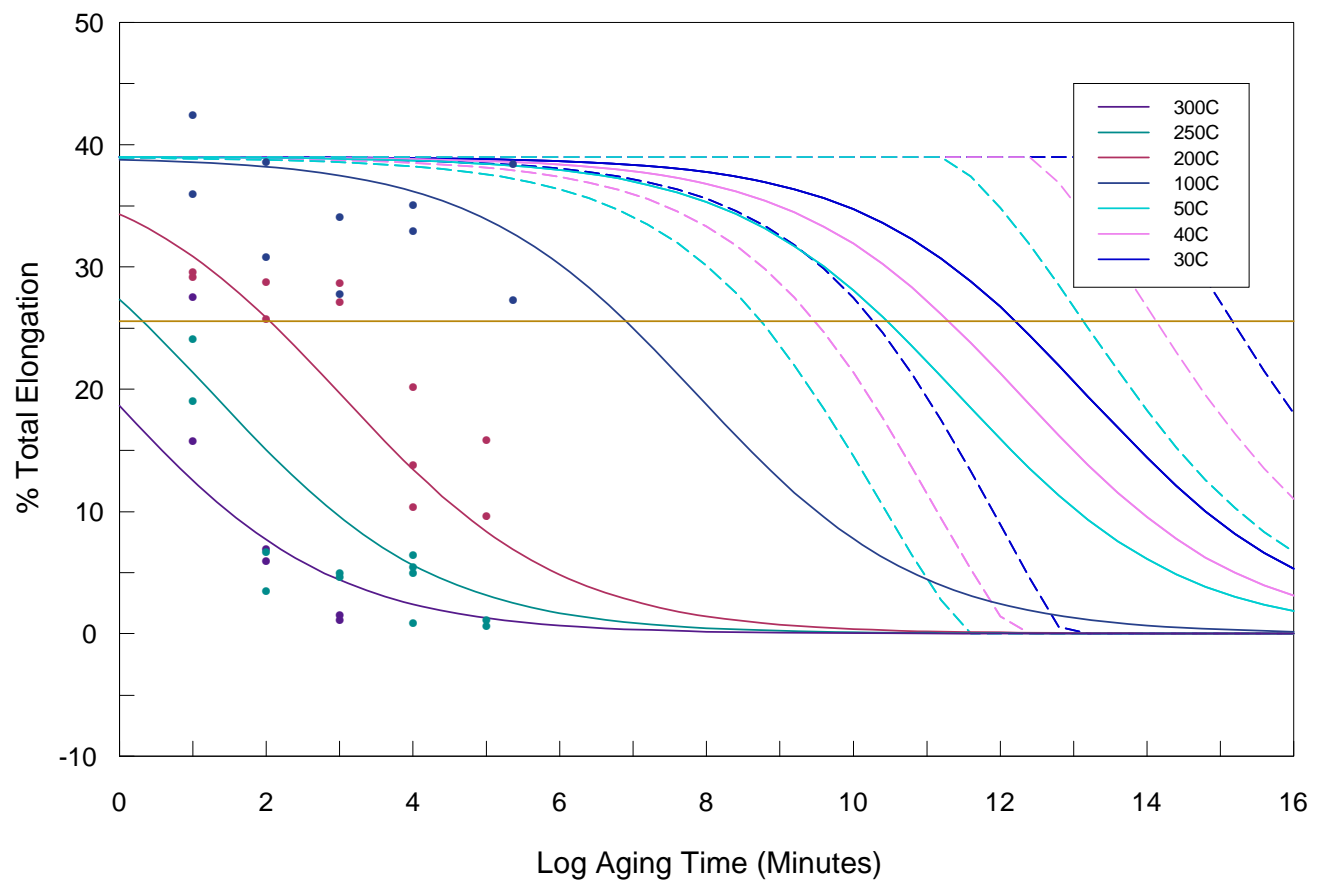

(b)

Figure 4.18. U-7.7Nb TE data (points), model fits to data (solid lines), and low-temperature model predictions (solid line - mean, dashed lines—95\% confidence intervals). (a) in terms of $f$, (b) in terms of absolute property. The yellow line is the failure threshold. 


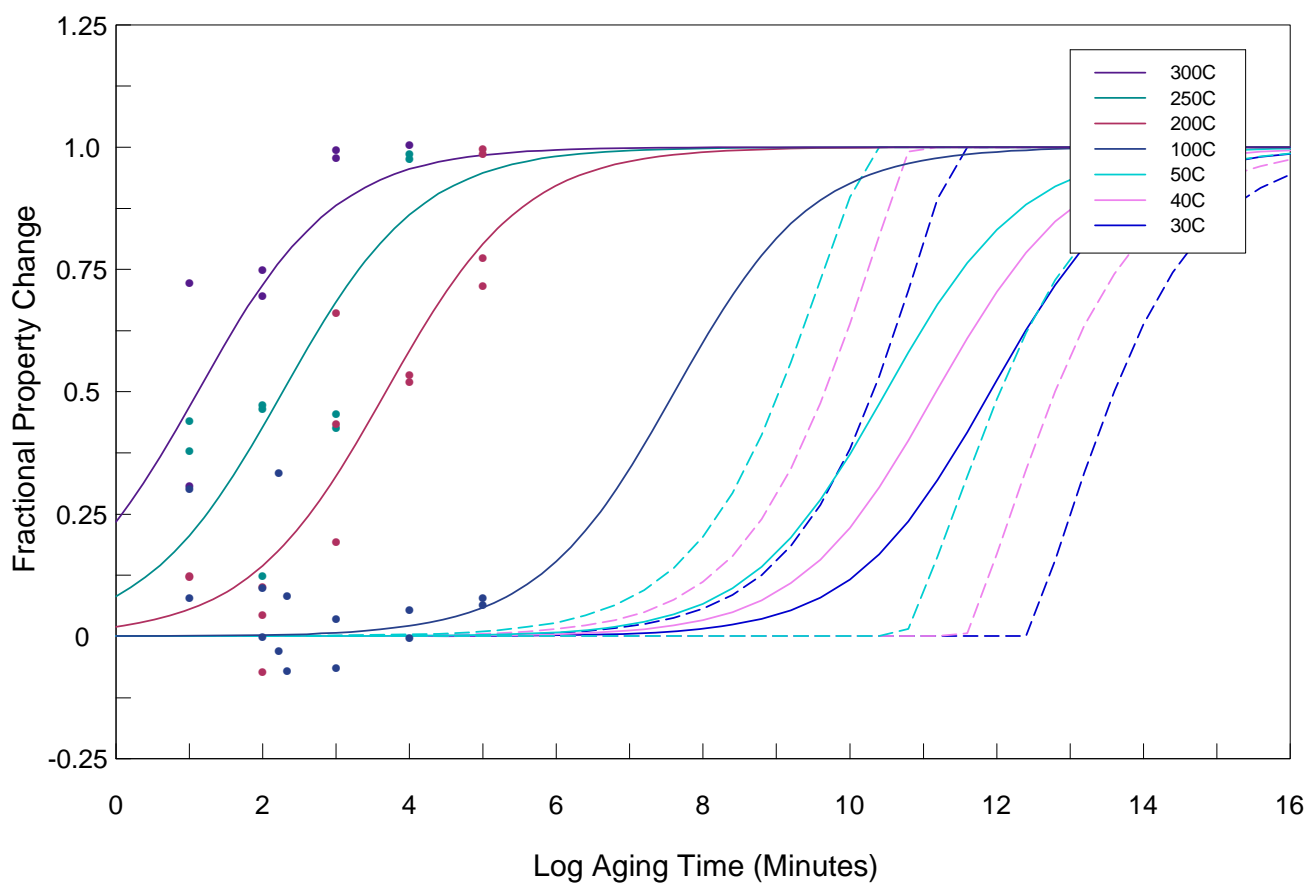

(a)

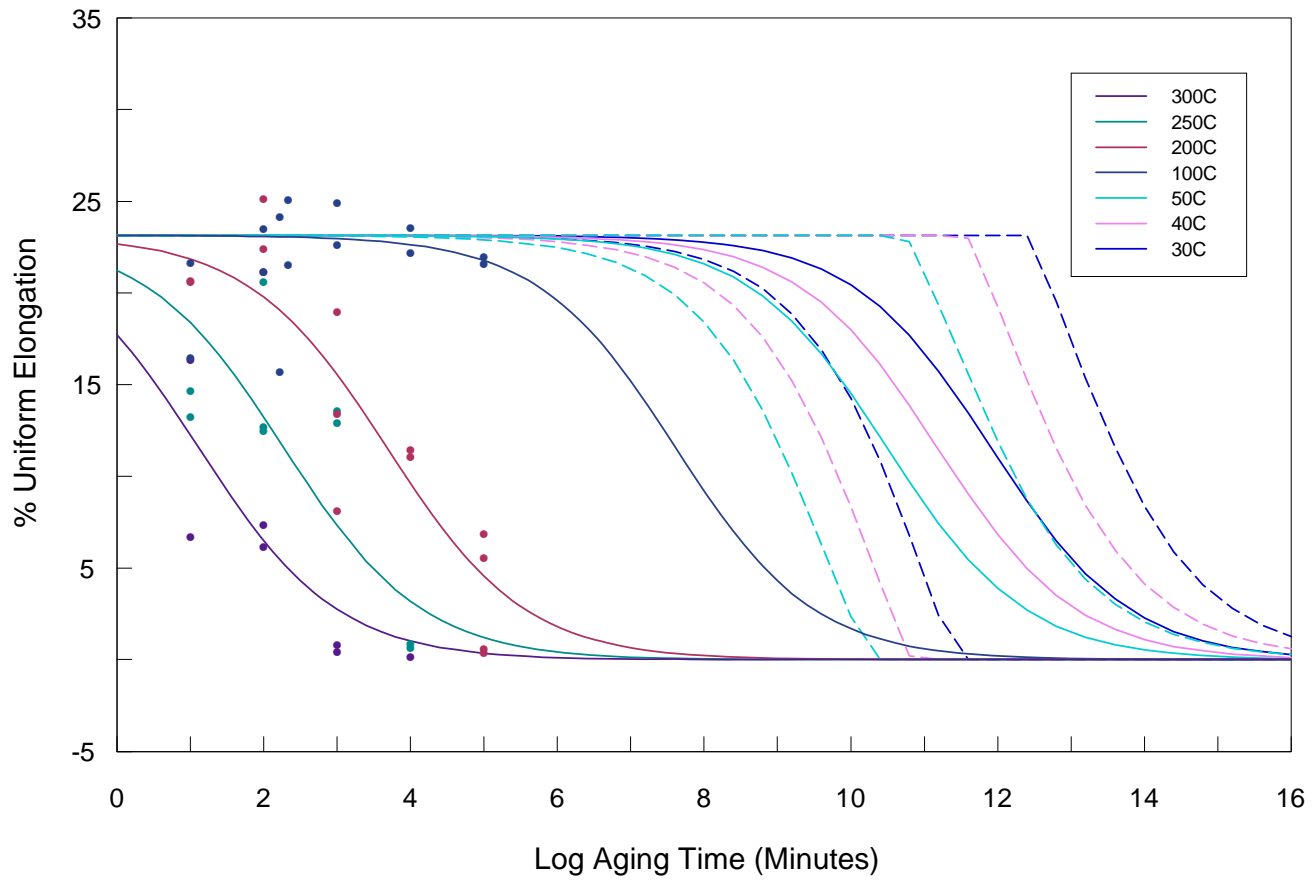

(b)

Figure 4.19. U-5.6Nb UE data (points), model fits to data (solid lines), and low-temperature model predictions (solid line-mean, dashed lines_-95\% confidence intervals). (a) in terms of $f$, (b) in terms of absolute property. 


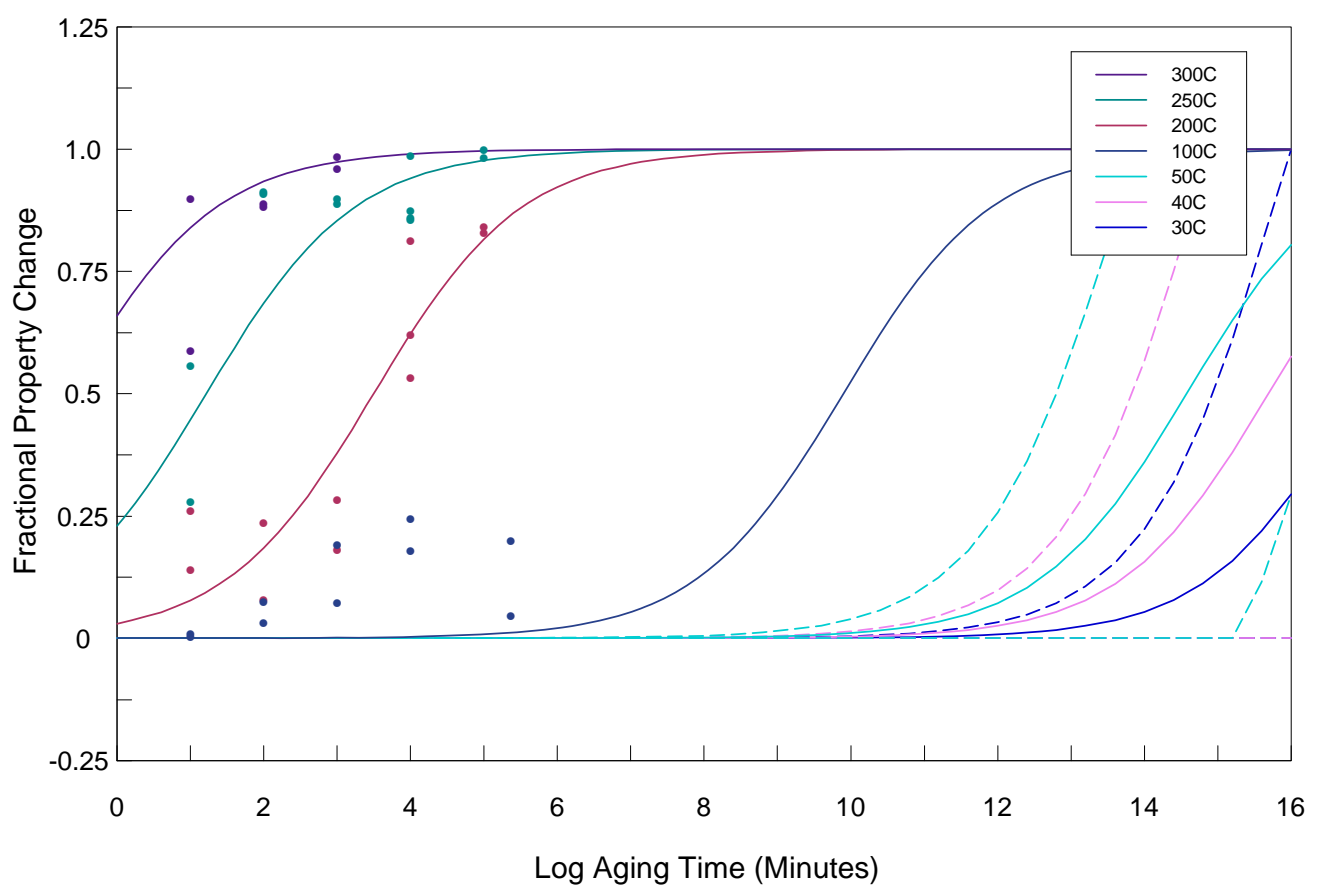

(a)

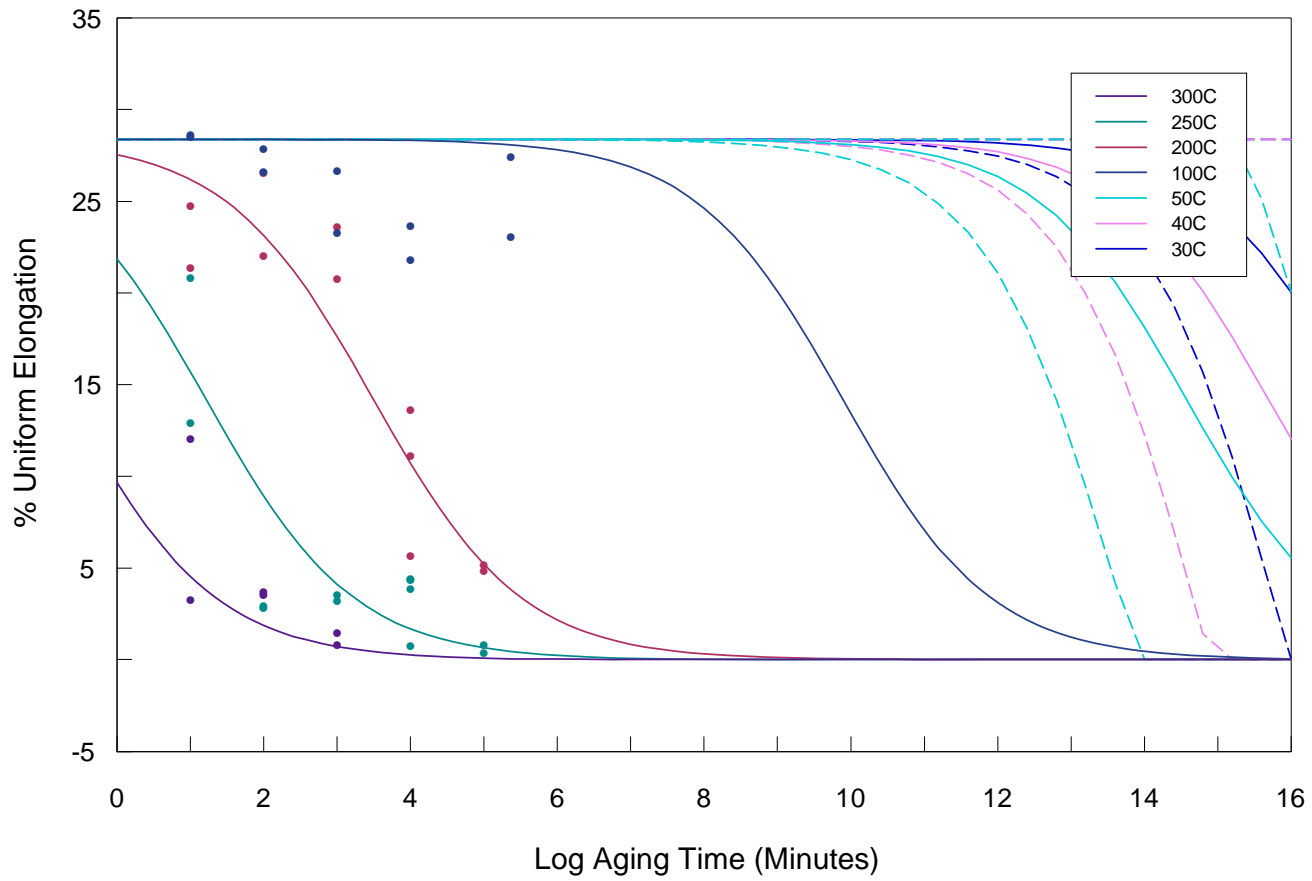

(b)

Figure 4.20. U-7.7Nb UE data (points), model fits to data (solid lines), and low-temperature model predictions (solid line-mean, dashed lines_-95\% confidence intervals). (a) in terms of $f$, (b) in terms of absolute property. 


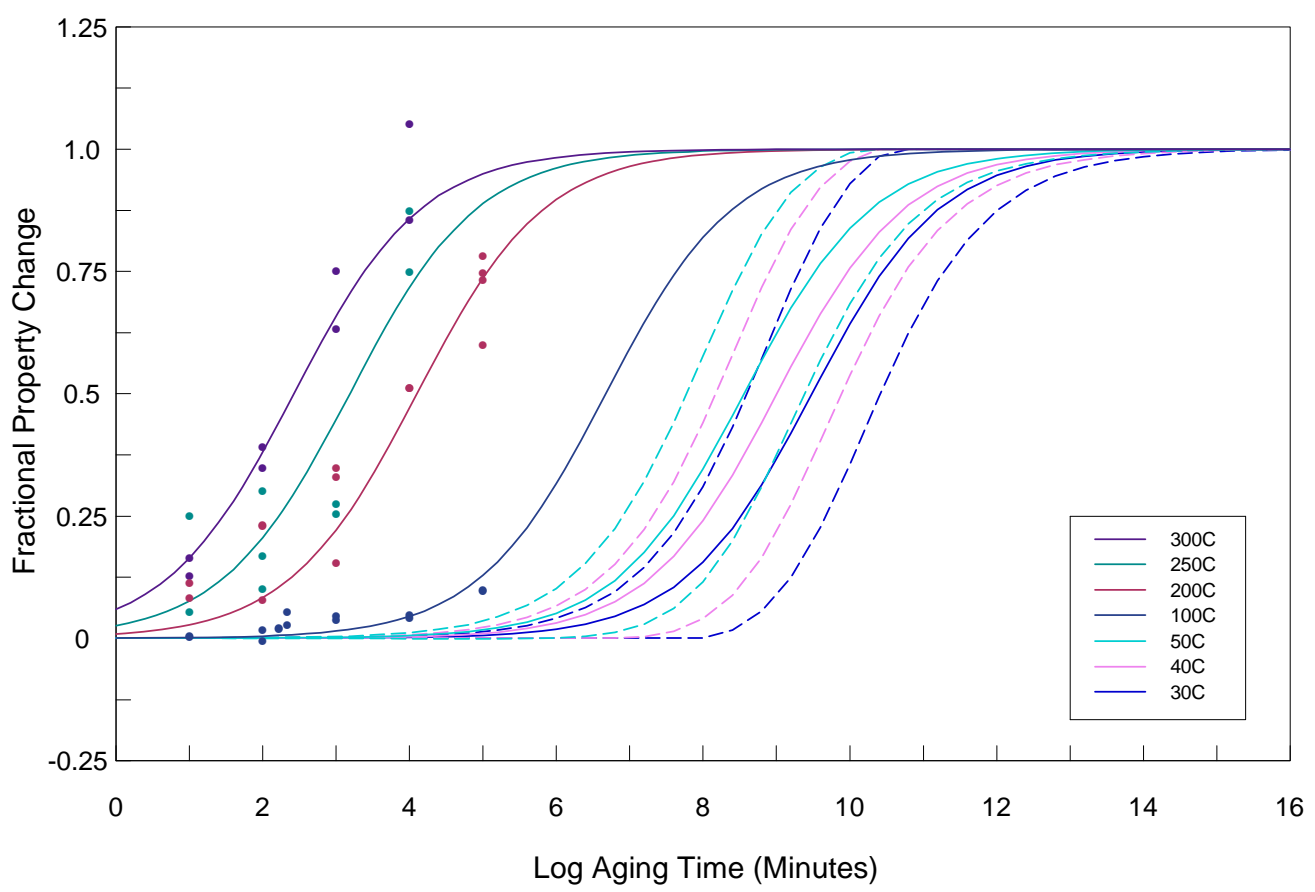

(a)

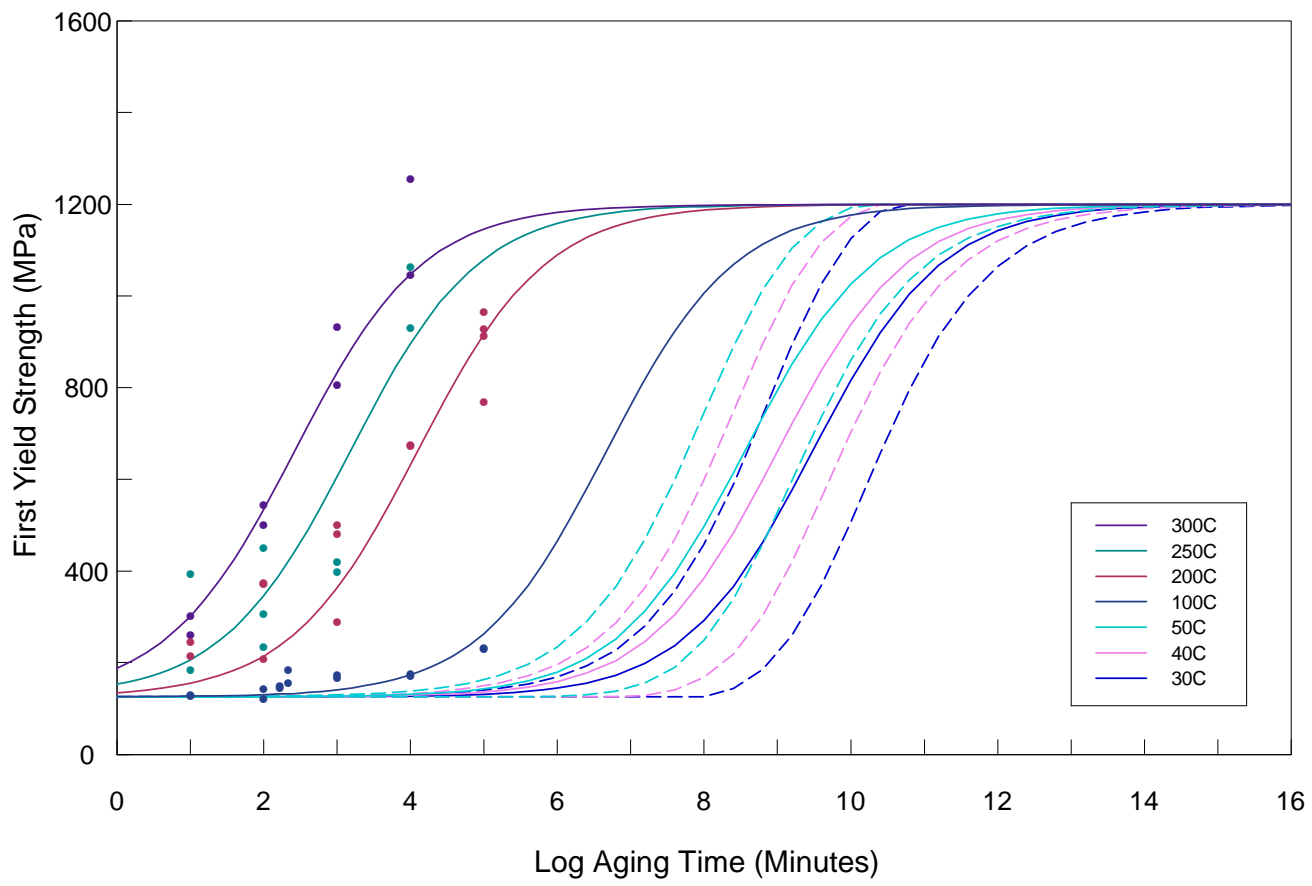

(b)

Figure 4.21. U-5.6Nb 1YS data (points), model fits to data (solid lines), and low-temperature model predictions (solid line-mean, dashed lines—95\% confidence intervals). (a) in terms of $f$, (b) in terms of absolute property. 


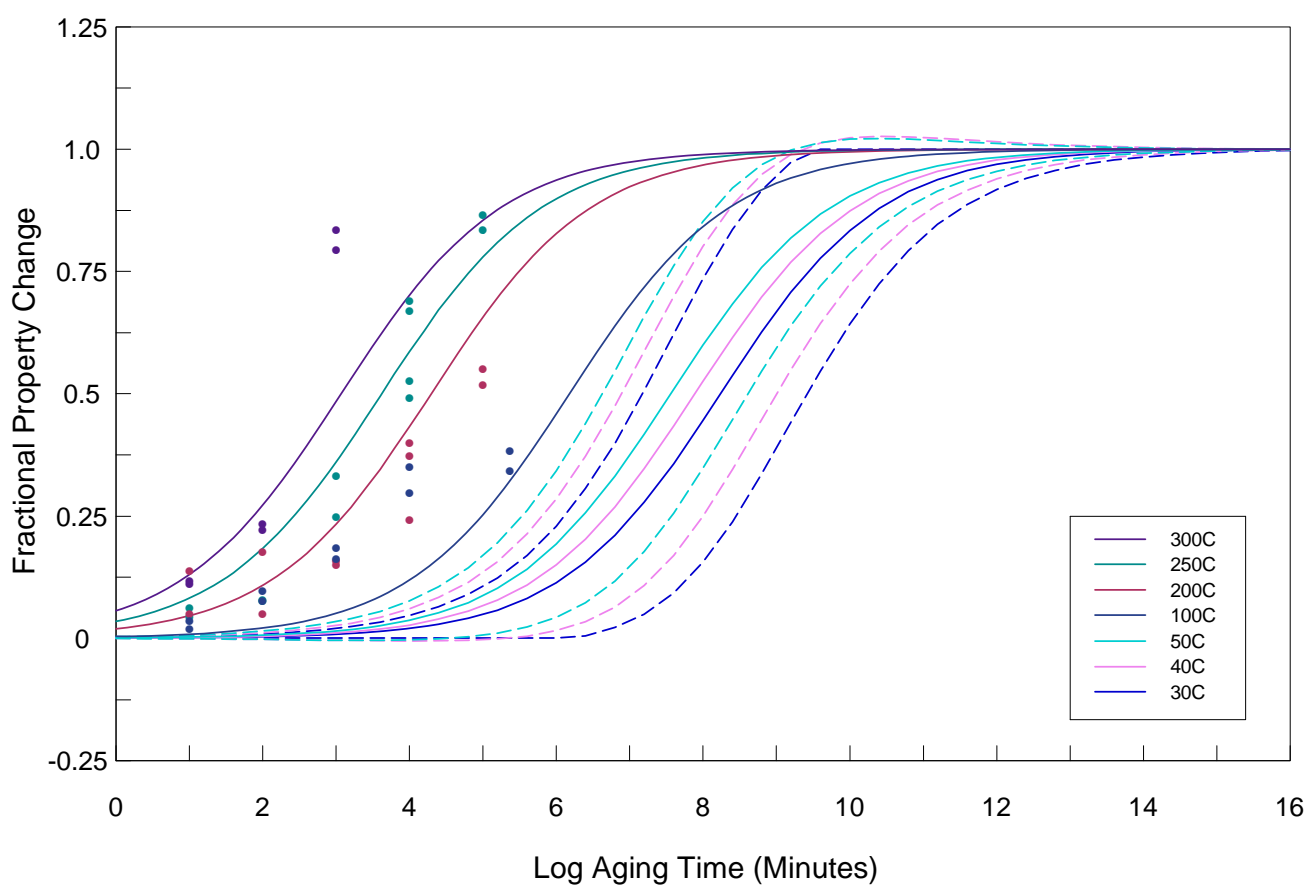

(a)

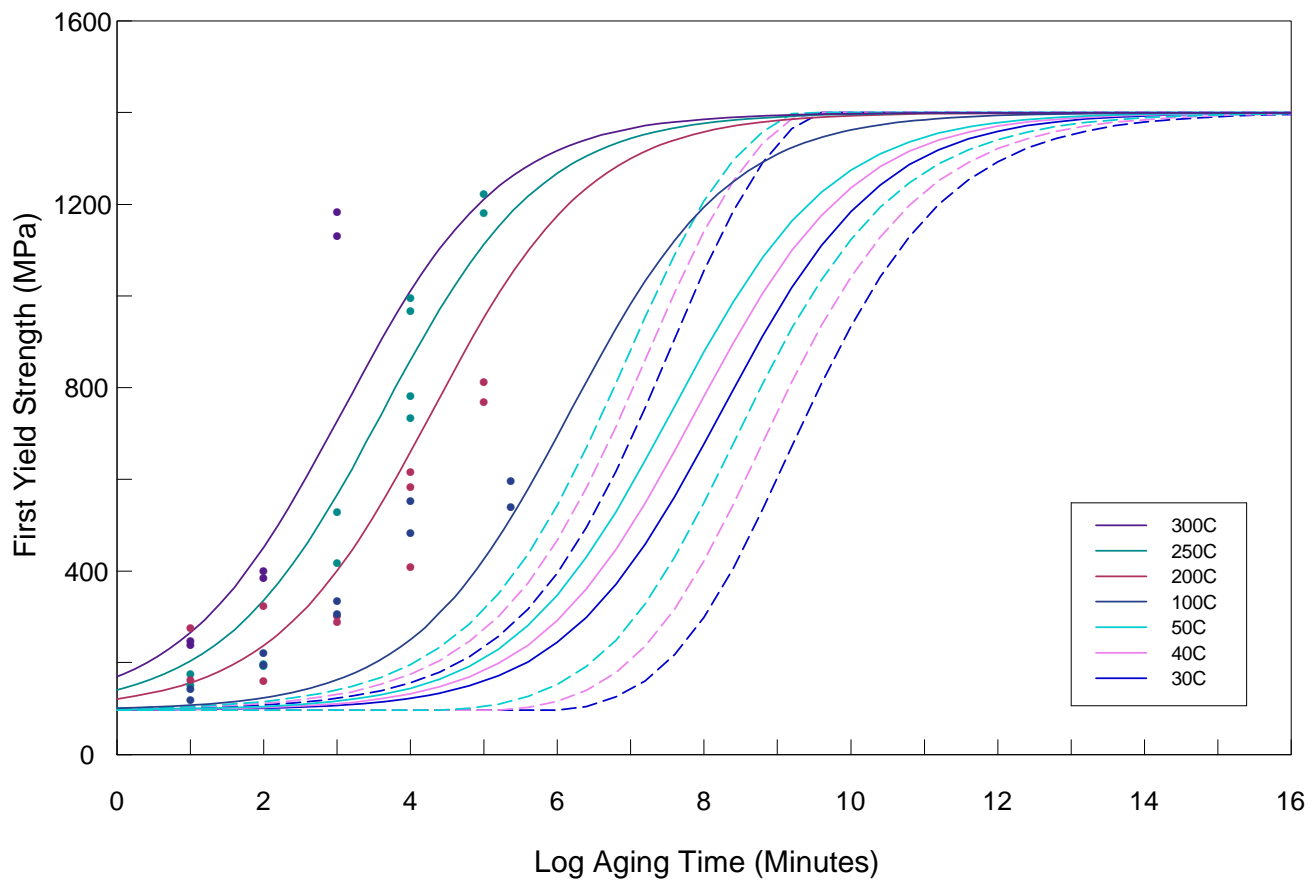

(b)

Figure 4.22. U-7.7Nb 1YS data (points), model fits to data (solid lines), and low-temperature model predictions (solid line-mean, dashed lines—95\% confidence intervals). (a) in terms of $f$, (b) in terms of absolute property. 


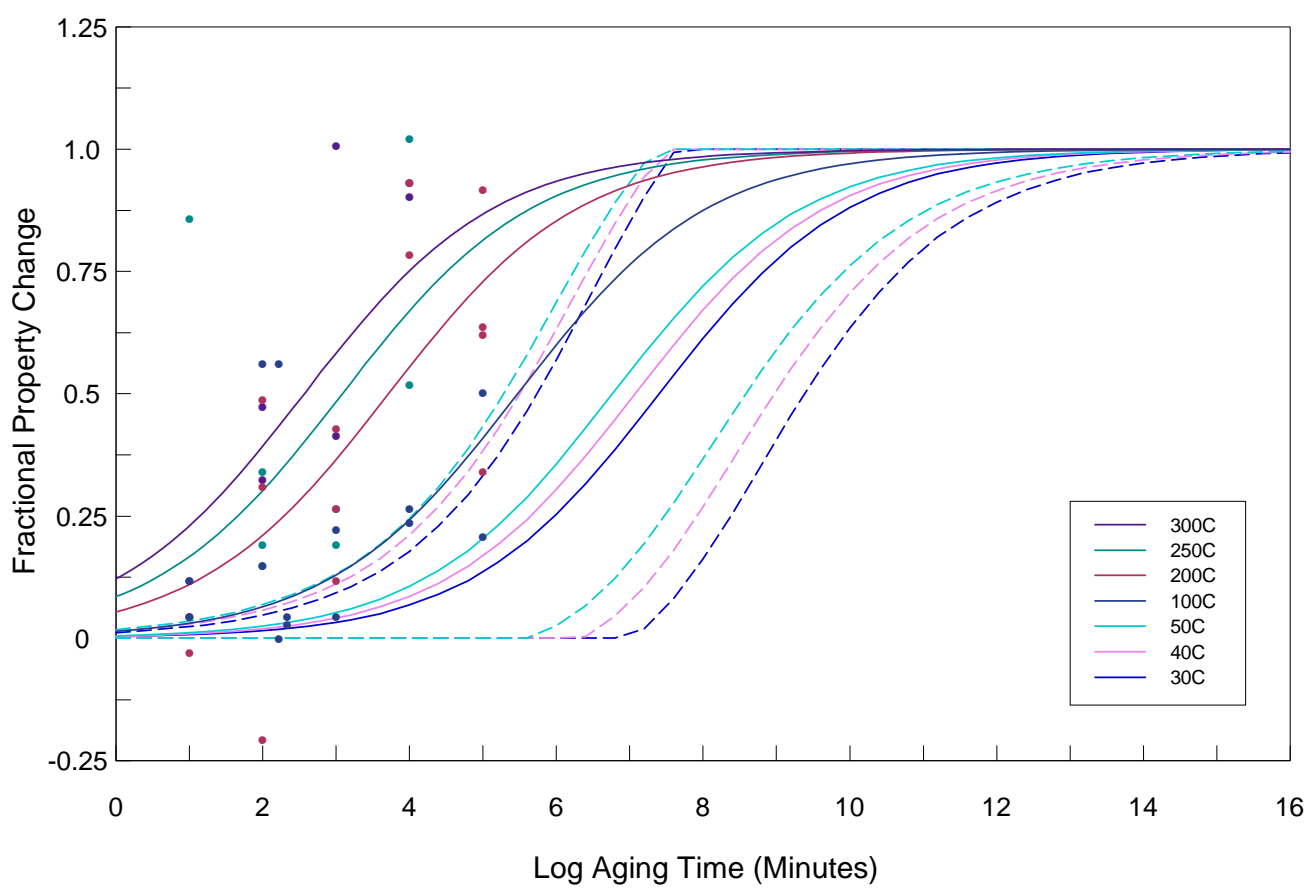

(a)

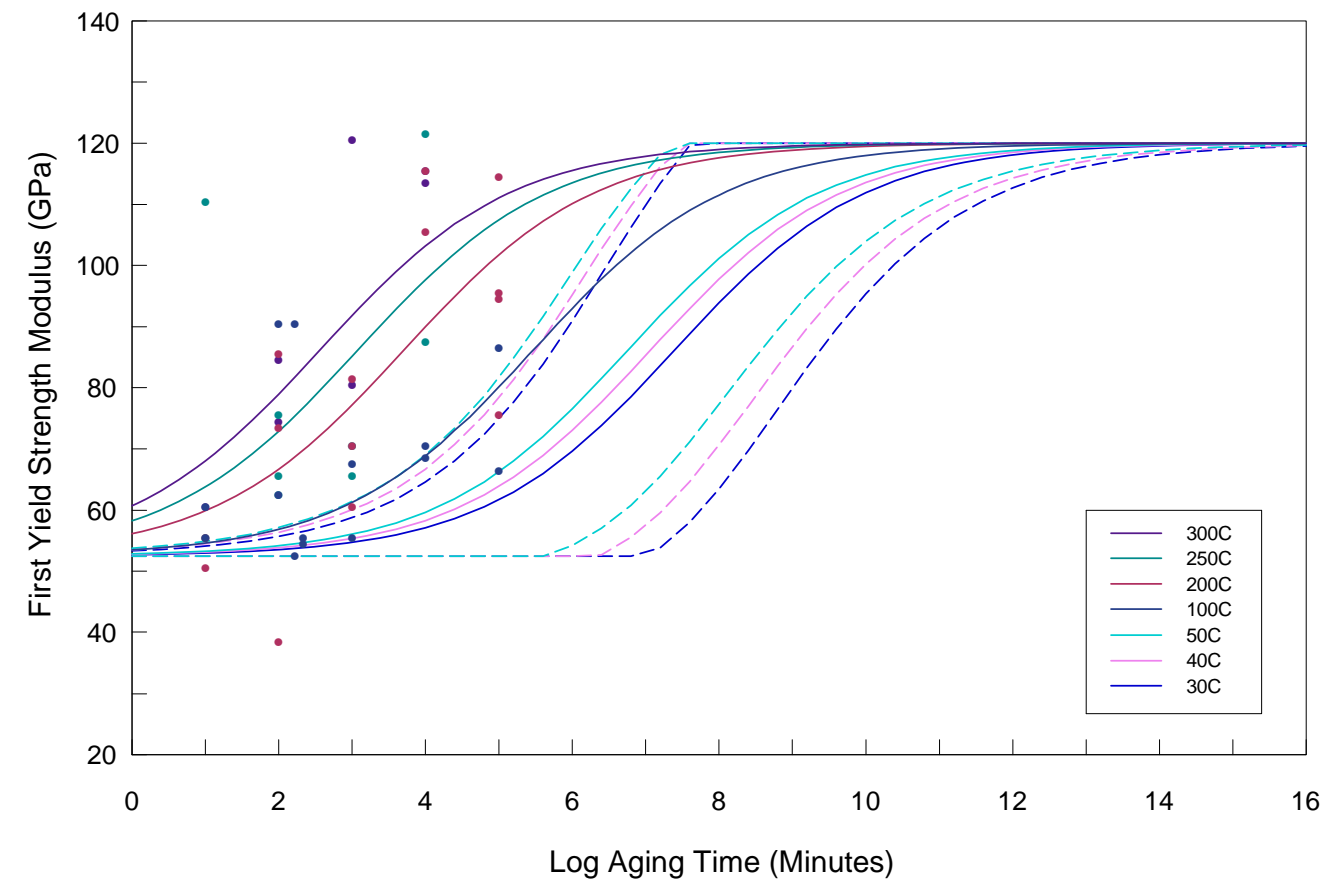

(b)

Figure 4.23. U-5.6Nb 1YM data (points), model fits to data (solid lines), and low-temperature model predictions (solid line-mean, dashed lines_-95\% confidence intervals). (a) in terms of $f$, (b) in terms of absolute property. 


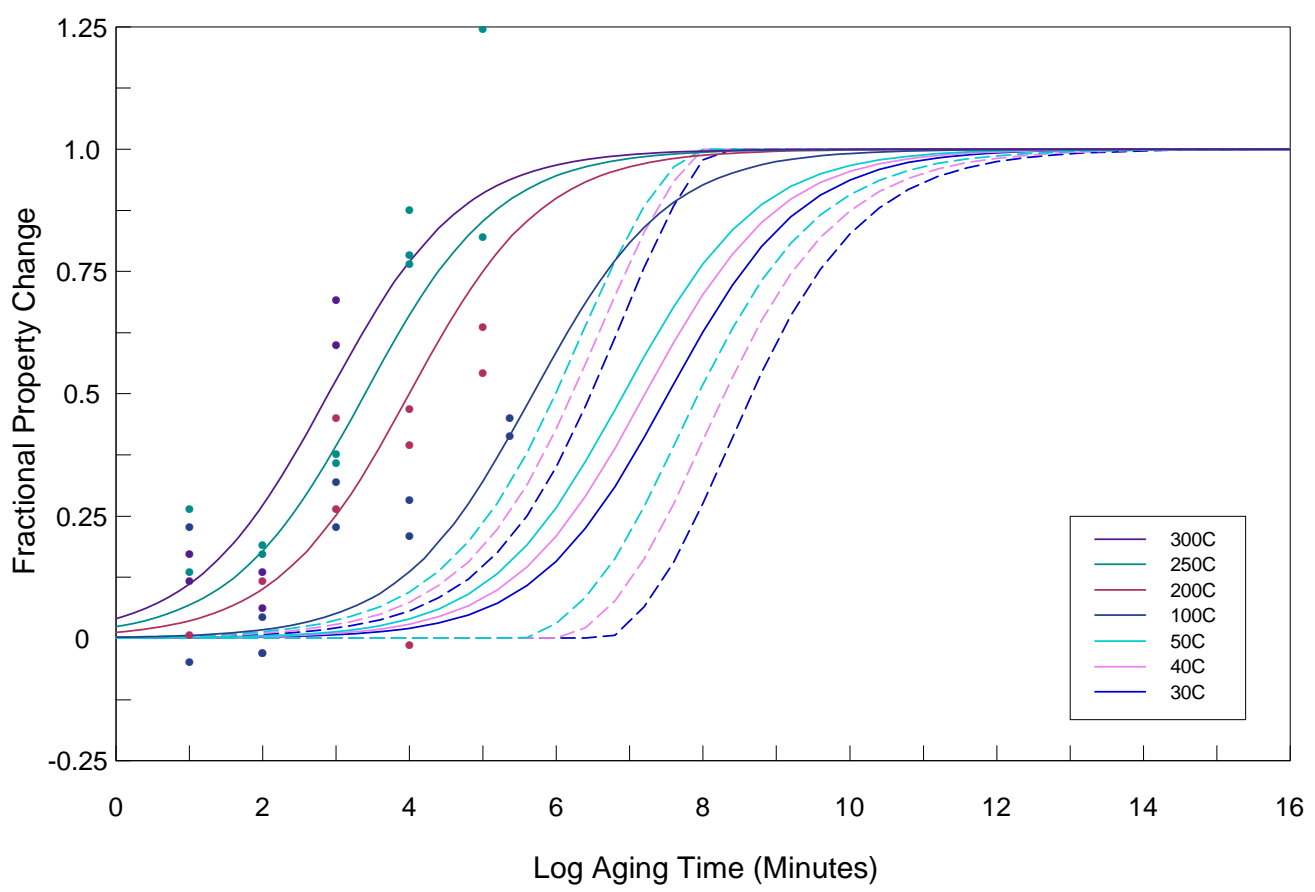

(a)

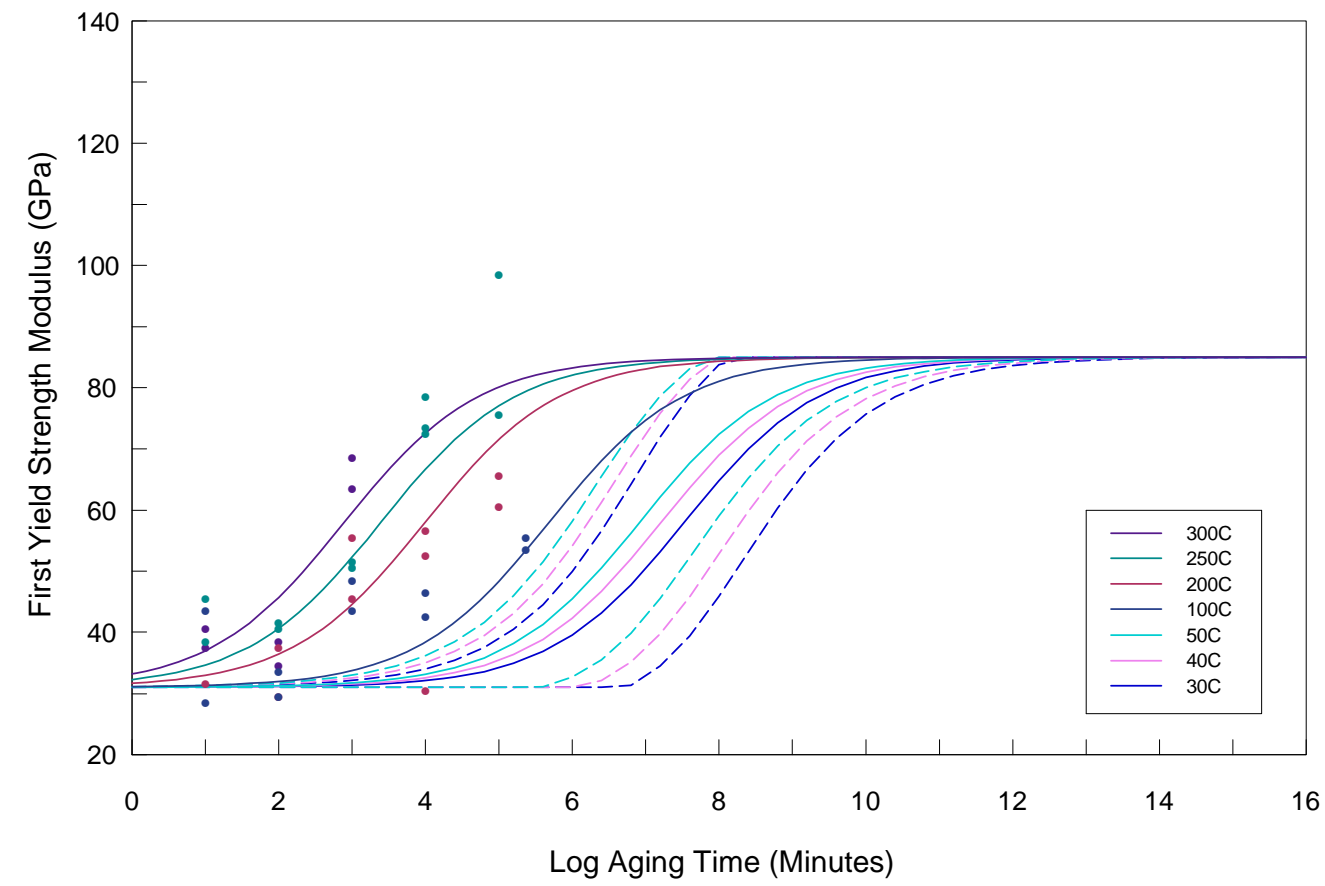

(b)

Figure 4.24. U-7.7Nb 1YM data (points), model fits to data (solid lines), and low-temperature model predictions (solid line — mean, dashed lines—95\% confidence intervals). (a) in terms of $f$, (b) in terms of absolute property. 


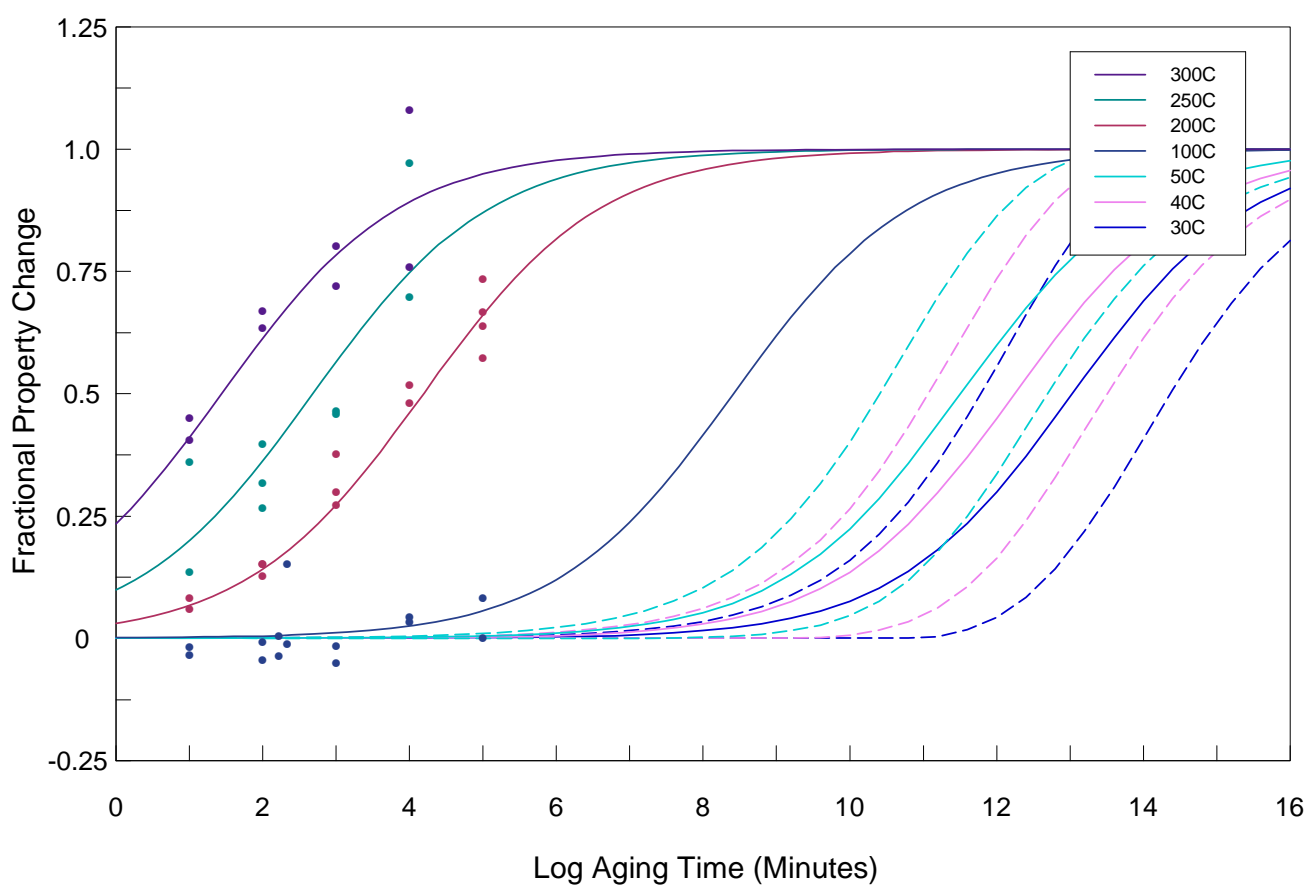

(a)

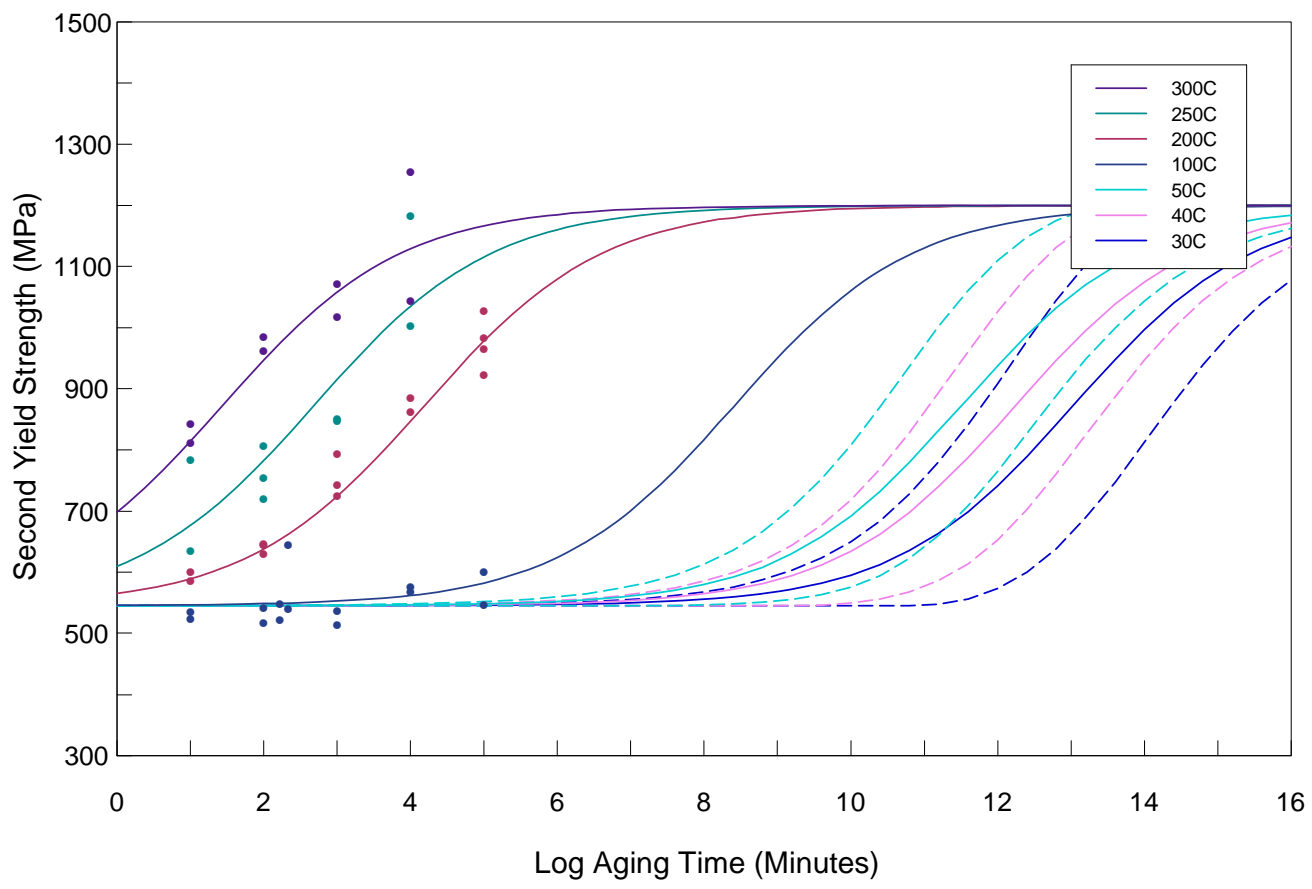

(b)

Figure 4.25. U-5.6Nb 2YS model data (points), model fits to data (solid lines), and lowtemperature model predictions (solid line_-mean, dashed lines—95\% confidence intervals). (a) in terms of $f$, (b) in terms of absolute property. 


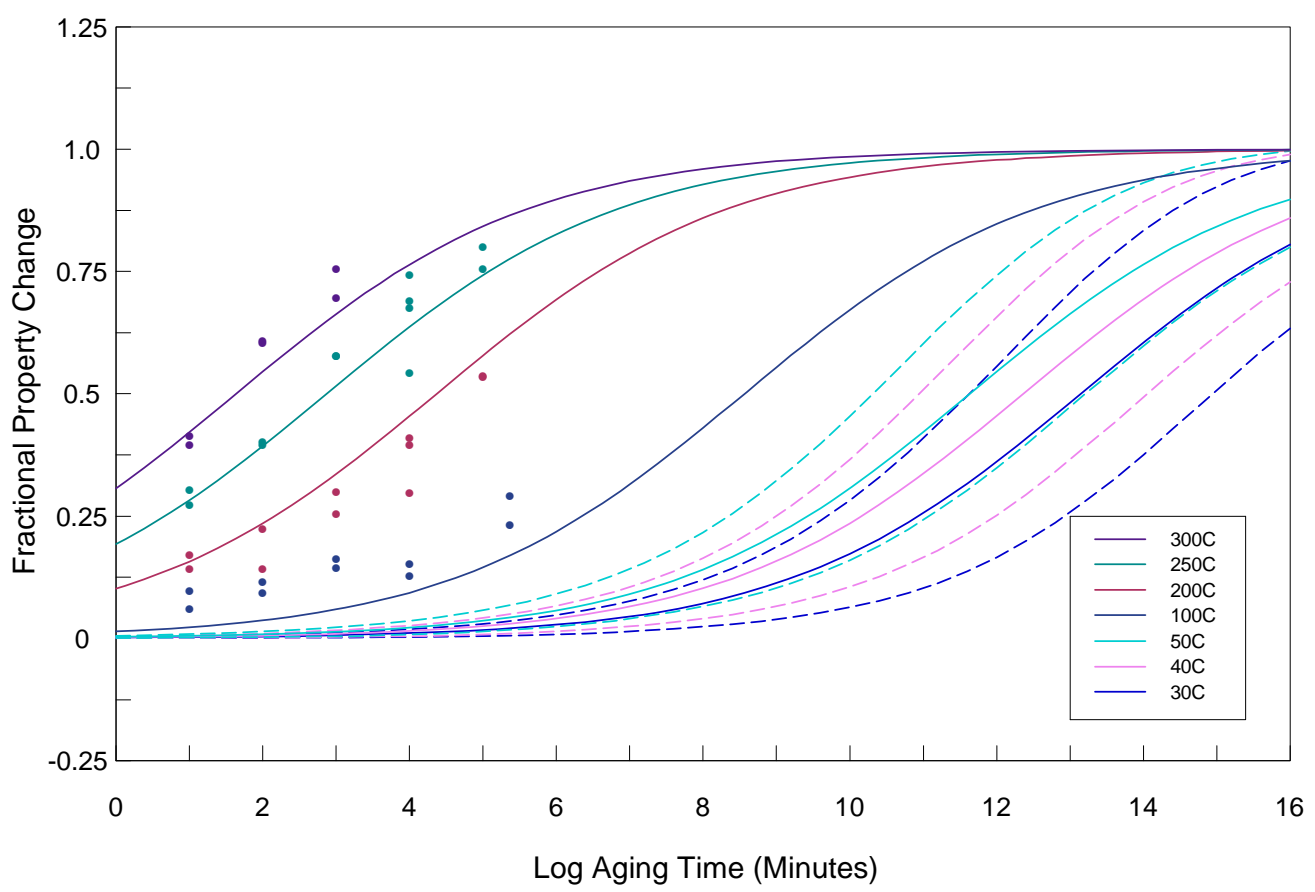

(a)

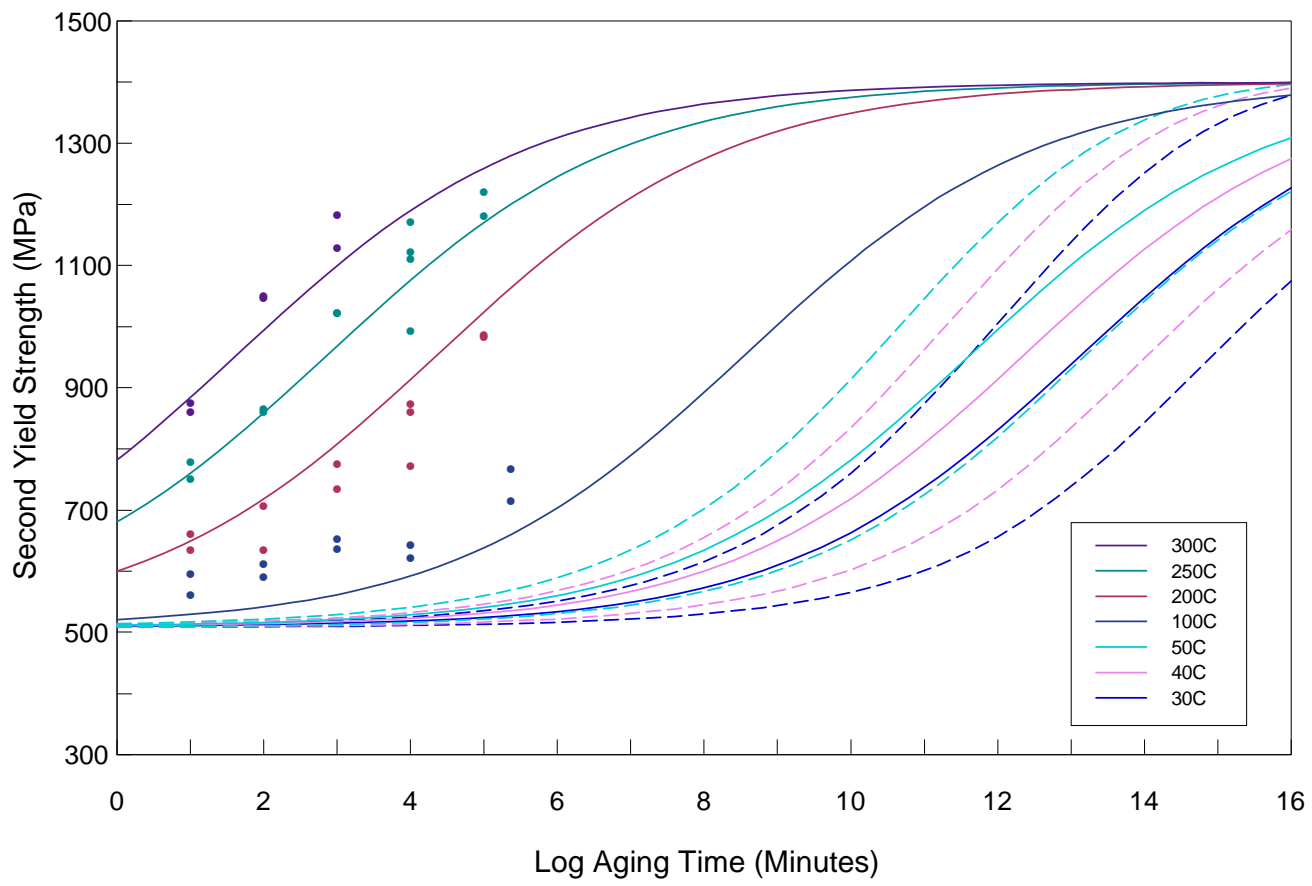

(b)

Figure 4.26. U-7.7Nb 2YS data (points), model fits to data (solid lines), and low-temperature model predictions (solid line-mean, dashed lines—95\% confidence intervals). (a) in terms of $f$, (b) in terms of absolute property. 


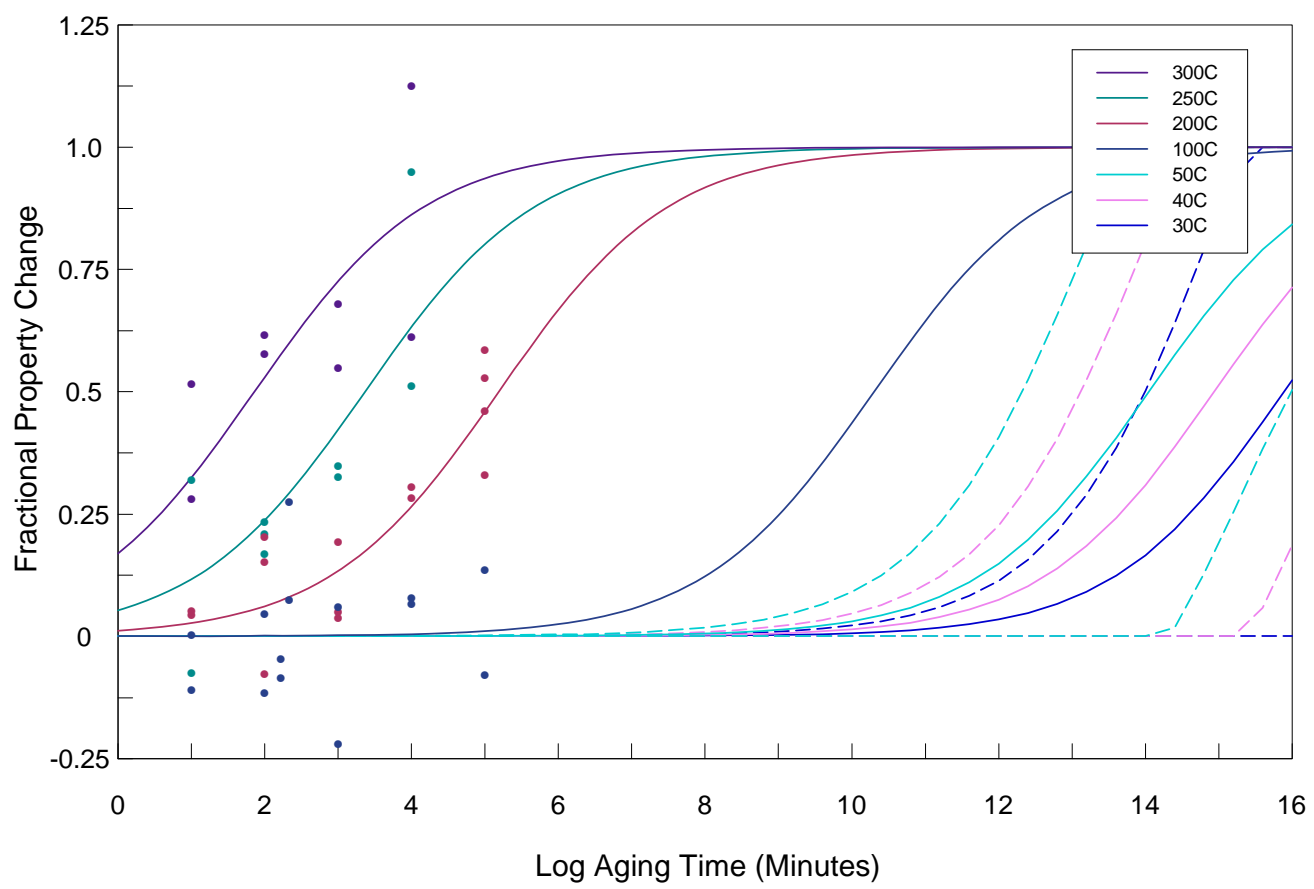

(a)

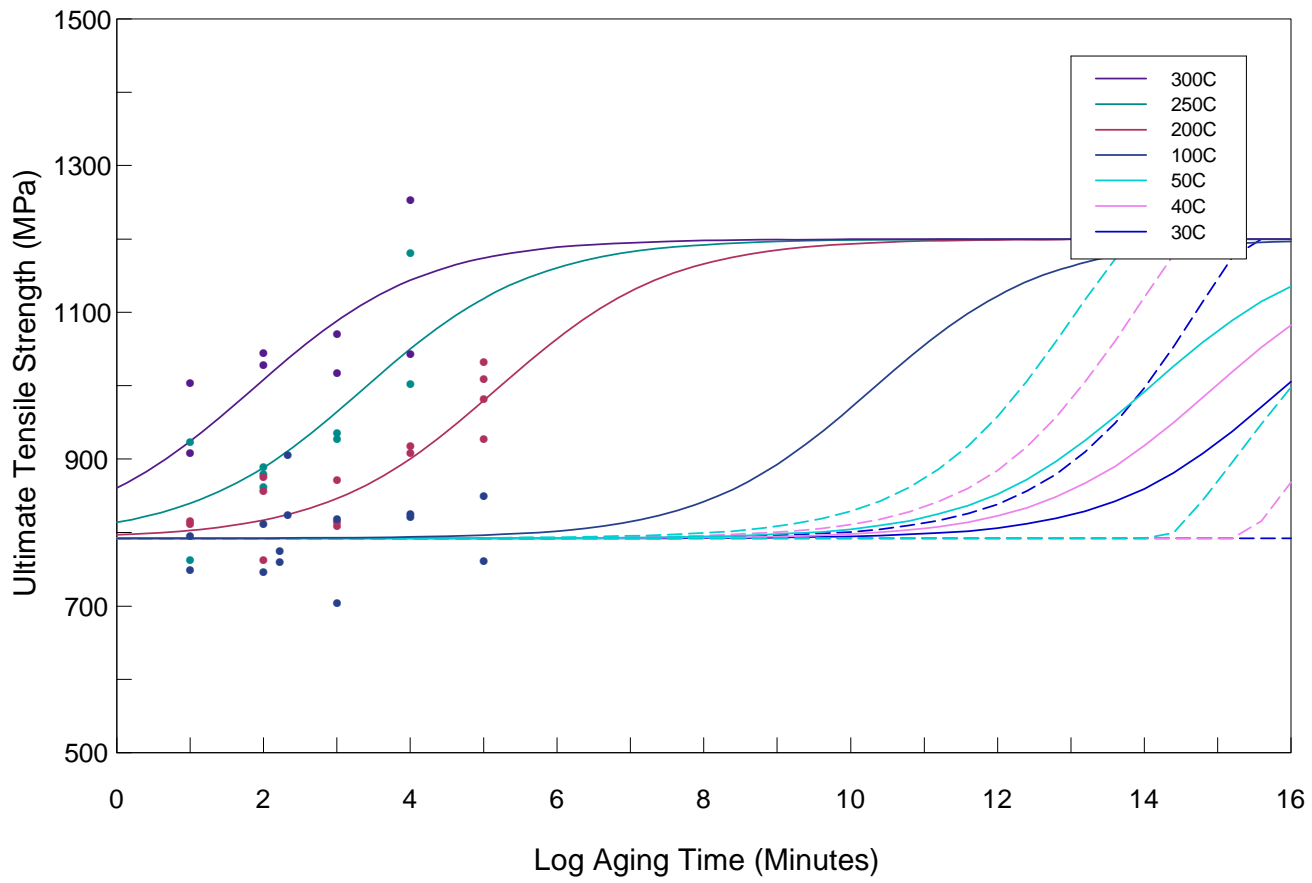

(b)

Figure 4.27. U-5.6Nb UTS data (points), model fits to data (solid lines), and low-temperature model predictions (solid line-mean, dashed lines—95\% confidence intervals). (a) in terms of $f$, (b) in terms of absolute property. 


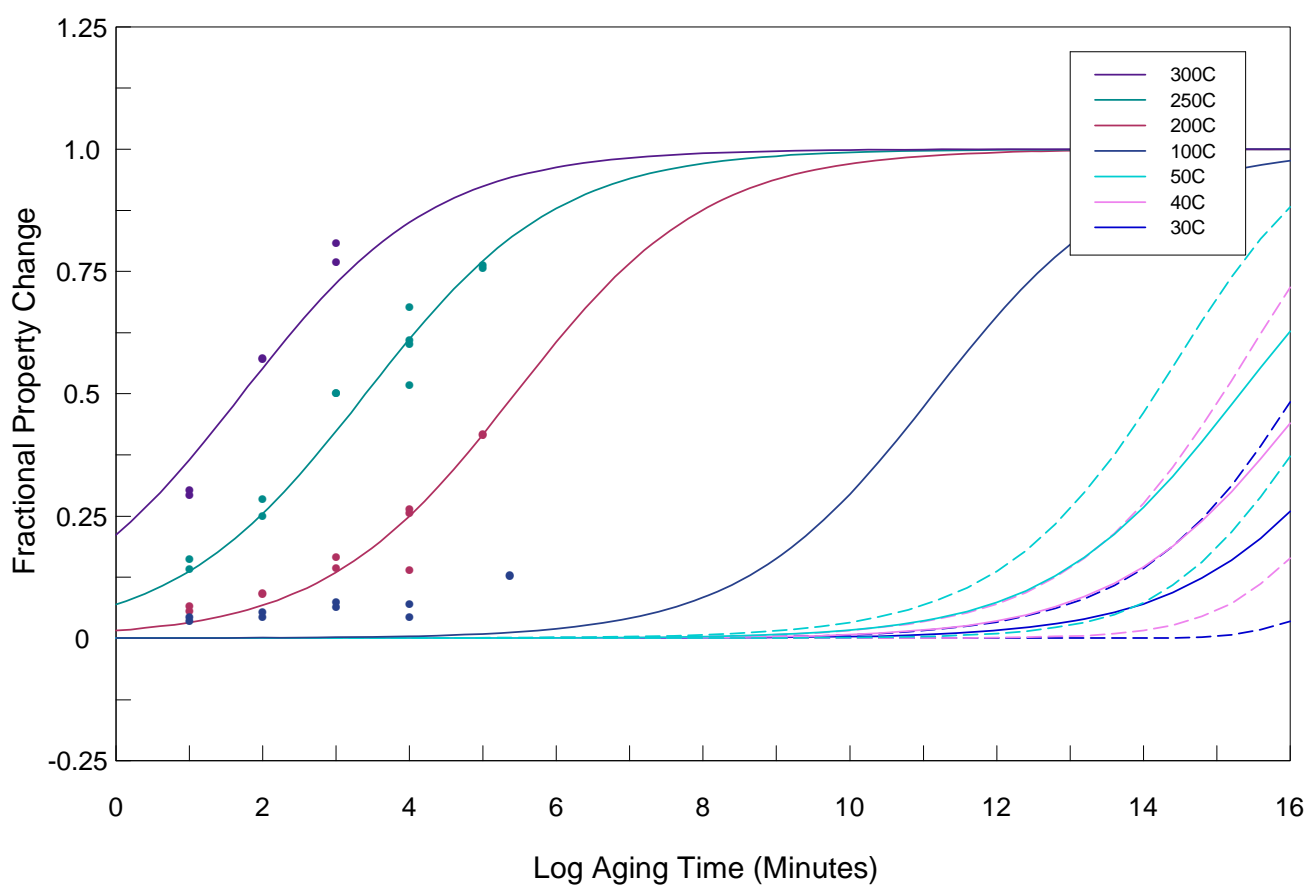

(a)

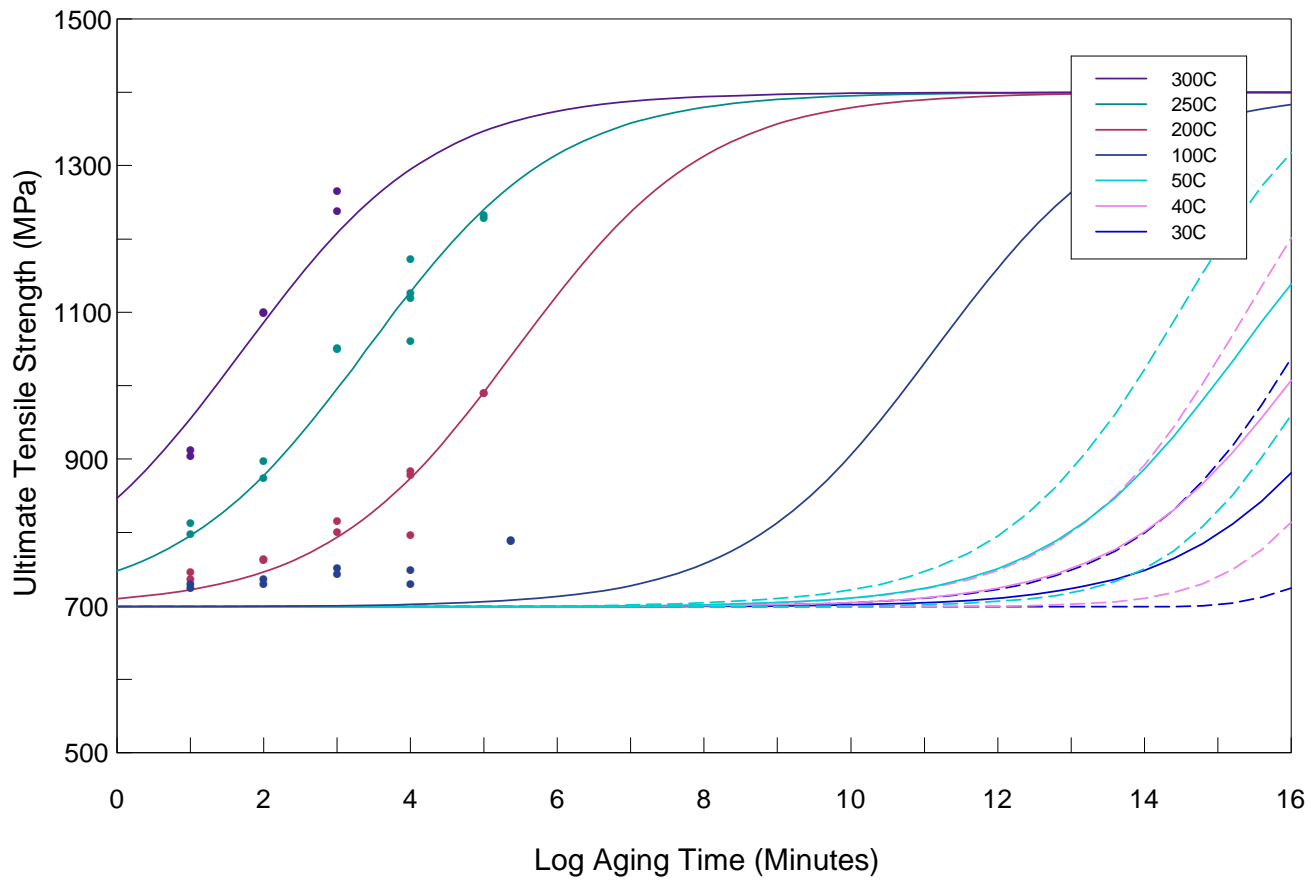

(b)

Figure 4.28. U-7.7Nb UTS data (points), model fits to data (solid lines), and low-temperature model predictions (solid line-mean, dashed lines—95\% confidence intervals). (a) in terms of $f$, (b) in terms of absolute property. 


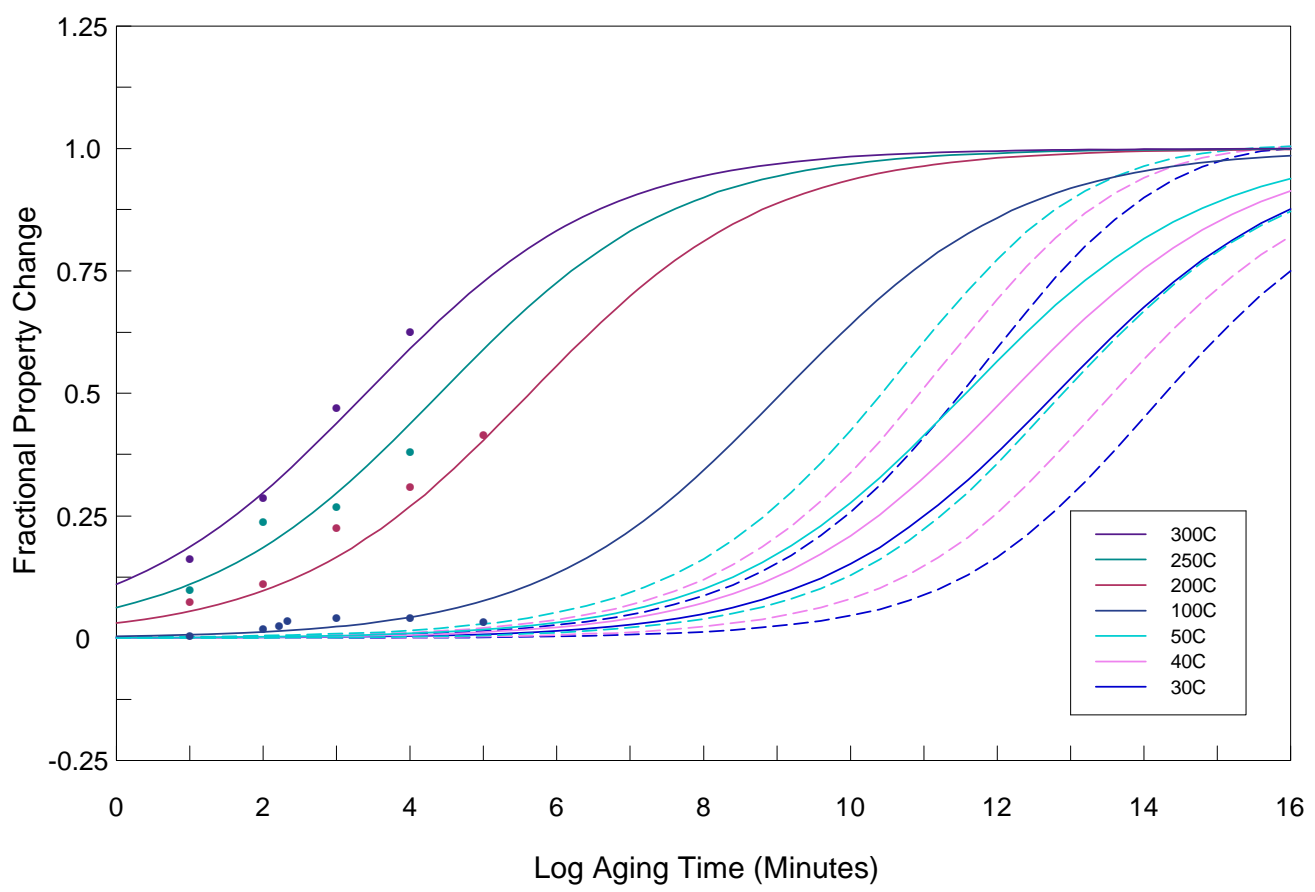

(a)

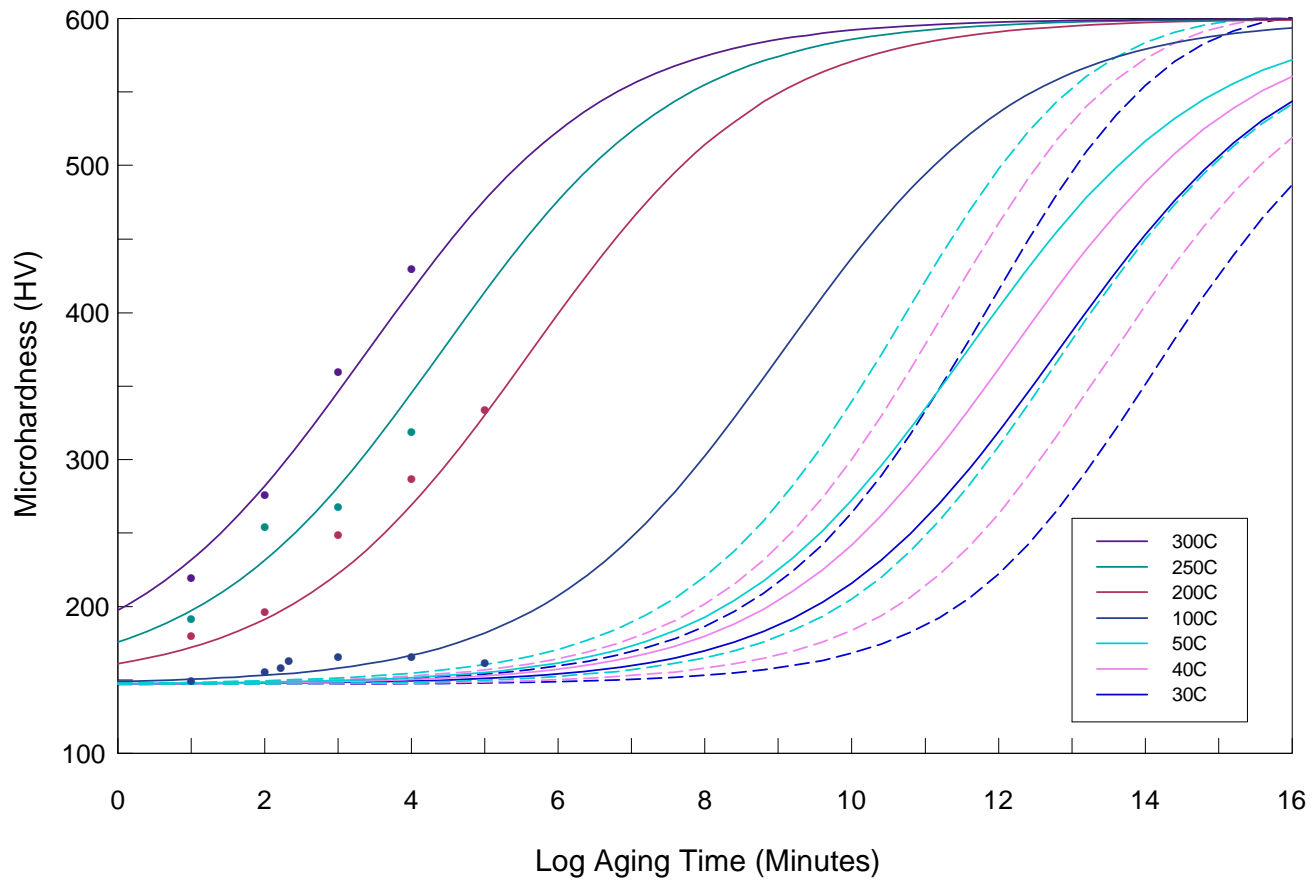

(b)

Figure 4.29. U-5.6Nb HV data (points), model fits to data (solid lines), and low-temperature model predictions (solid line-mean, dashed lines—95\% confidence intervals). (a) in terms of $f$, (b) in terms of absolute property. 


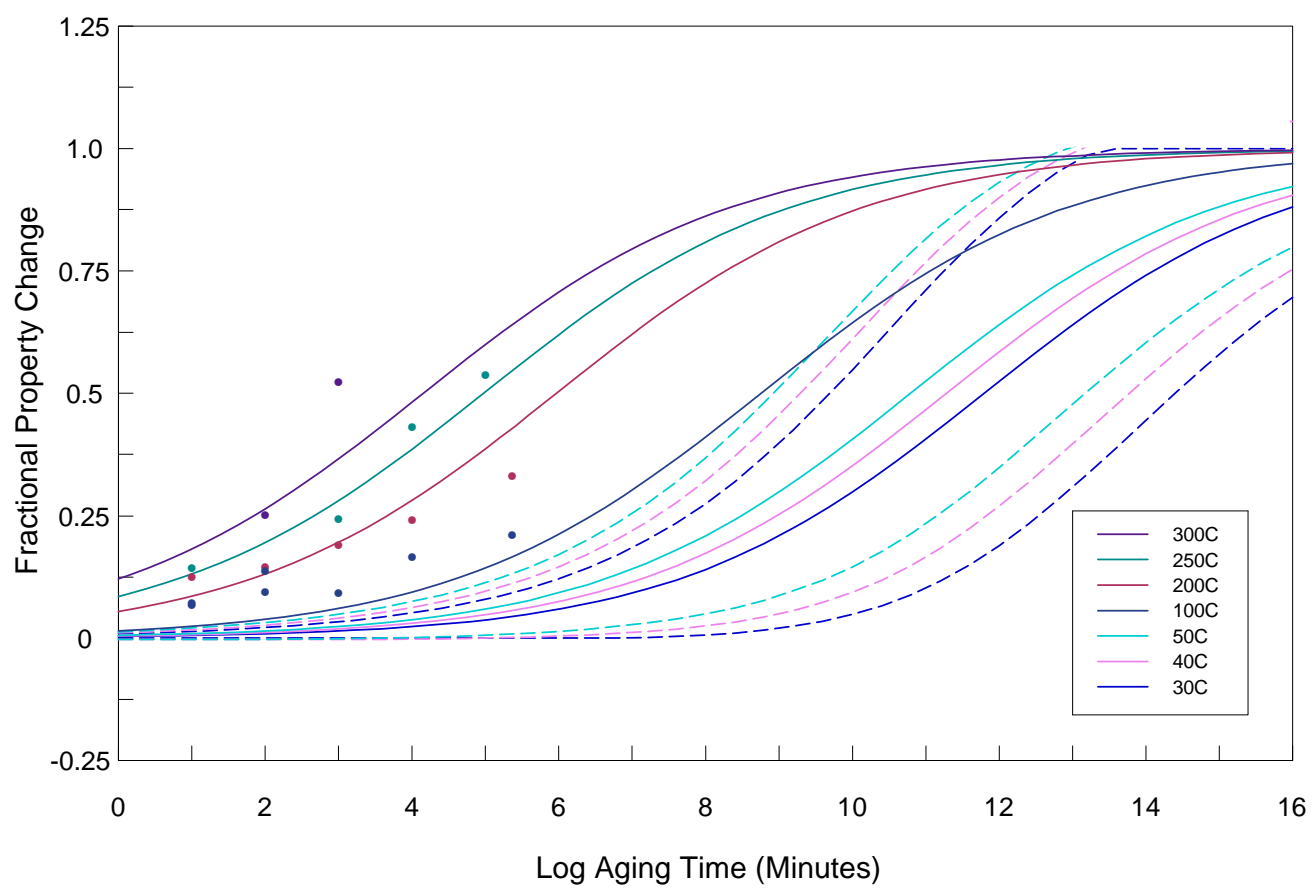

(a)

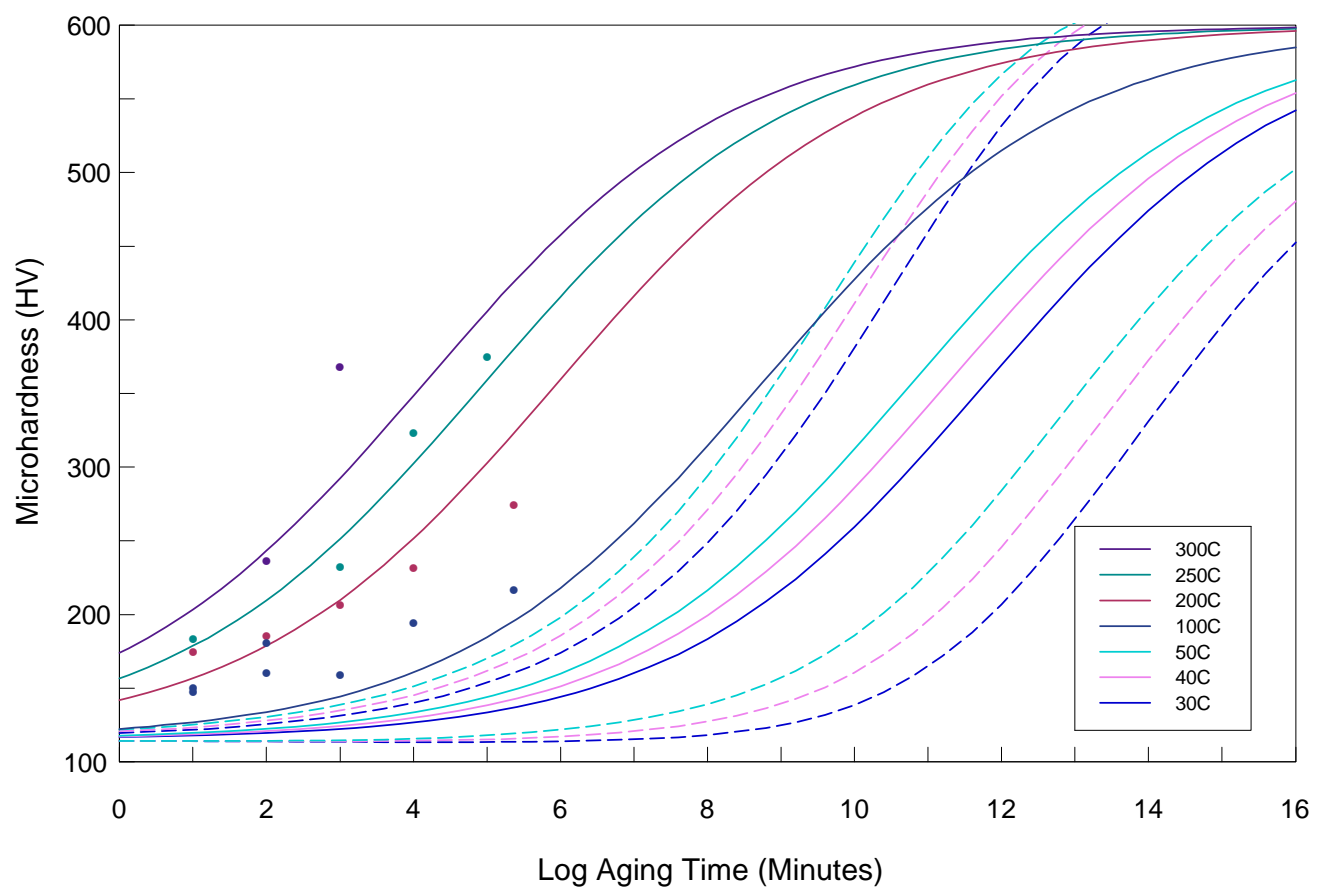

(b)

Figure 4.30. U-7.7Nb HV data (points), model fits to data (solid lines), and low-temperature model predictions (solid line-mean, dashed lines—95\% confidence intervals). (a) in terms of $f$, (b) in terms of absolute property. 


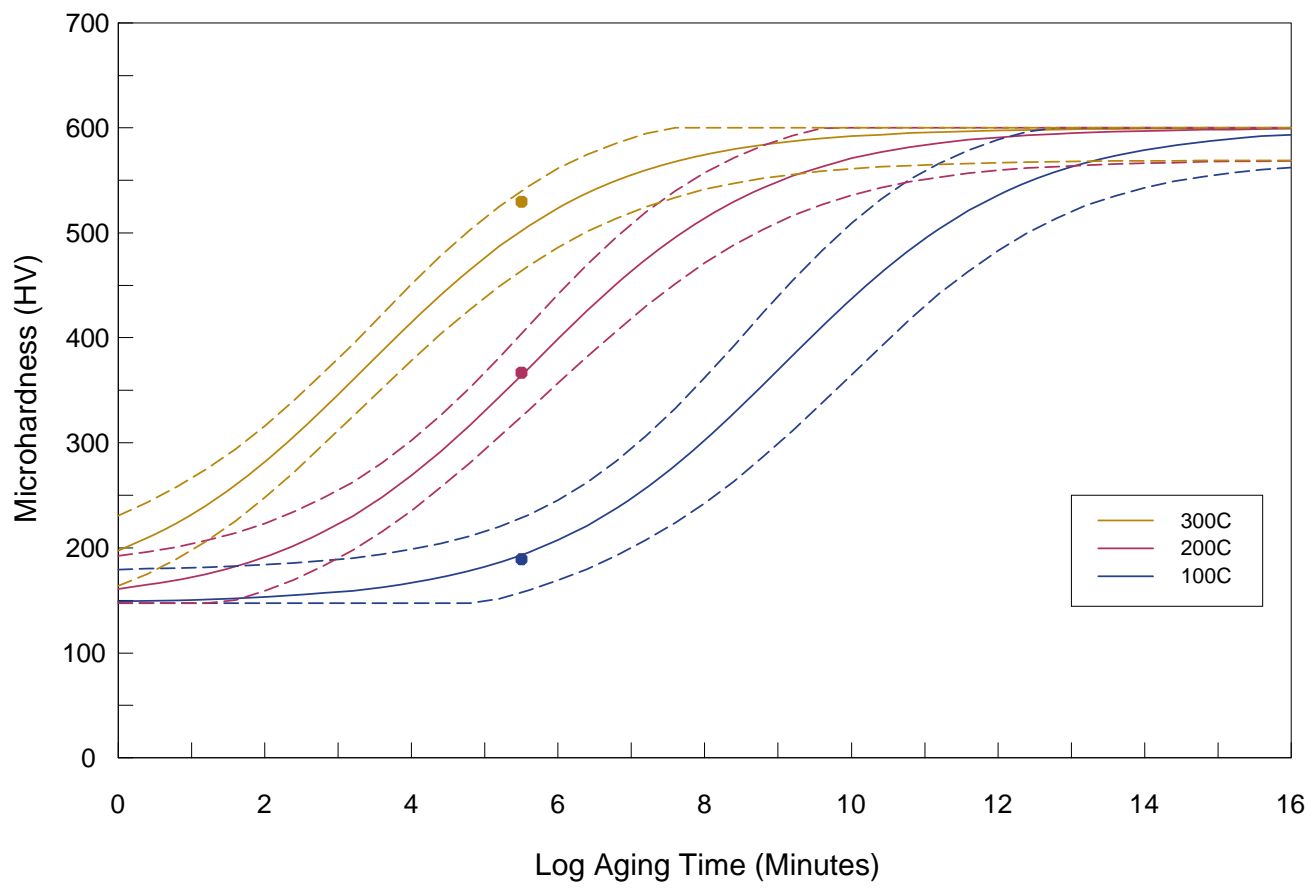

Figure 4.31. U-5.6Nb validation HV data (points) and model predictions (solid lines-mean, dashed lines_-95\% prediction intervals). 


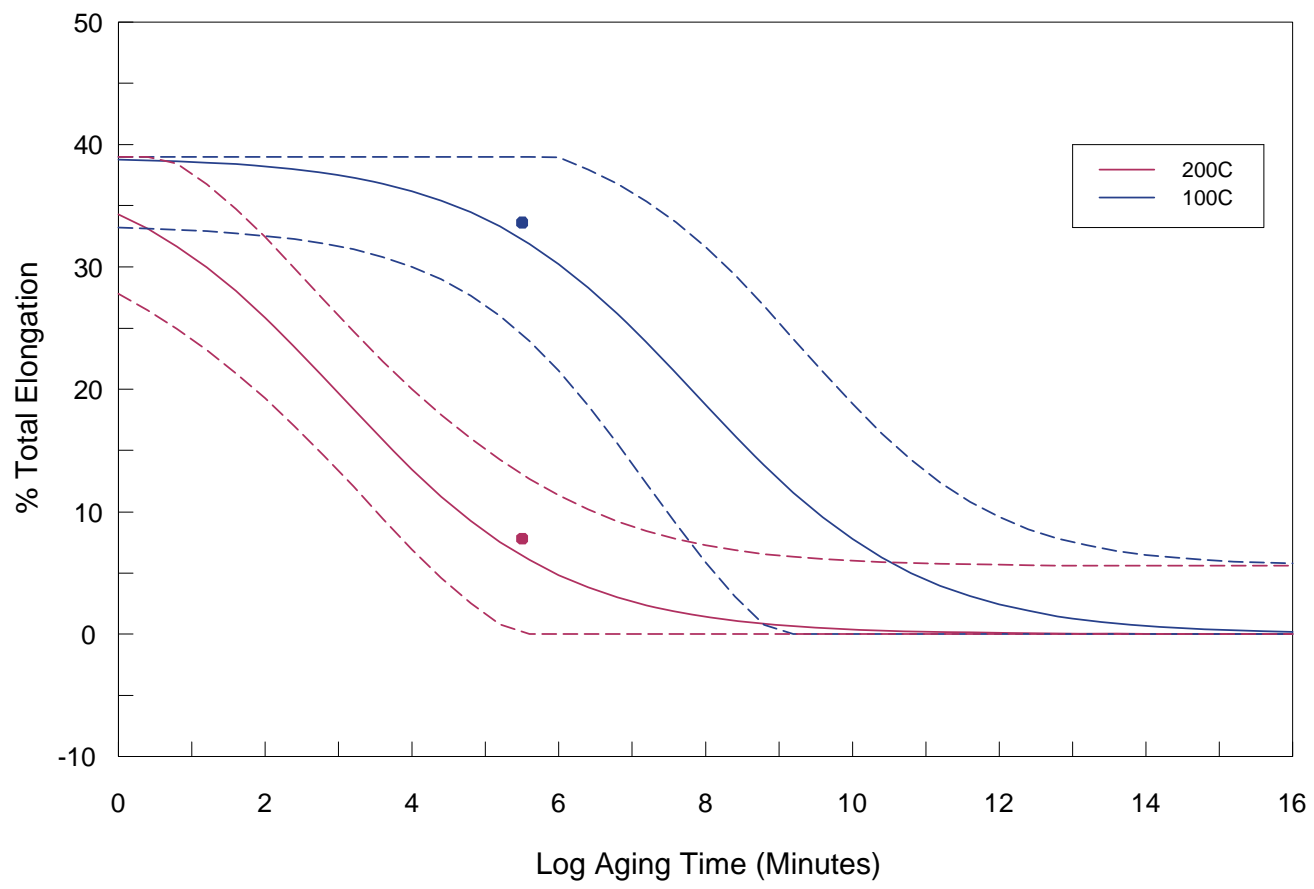

Figure 4.32. U-7.7Nb validation TE data (points) and model predictions (solid lines - mean, dashed lines-95\% prediction intervals).

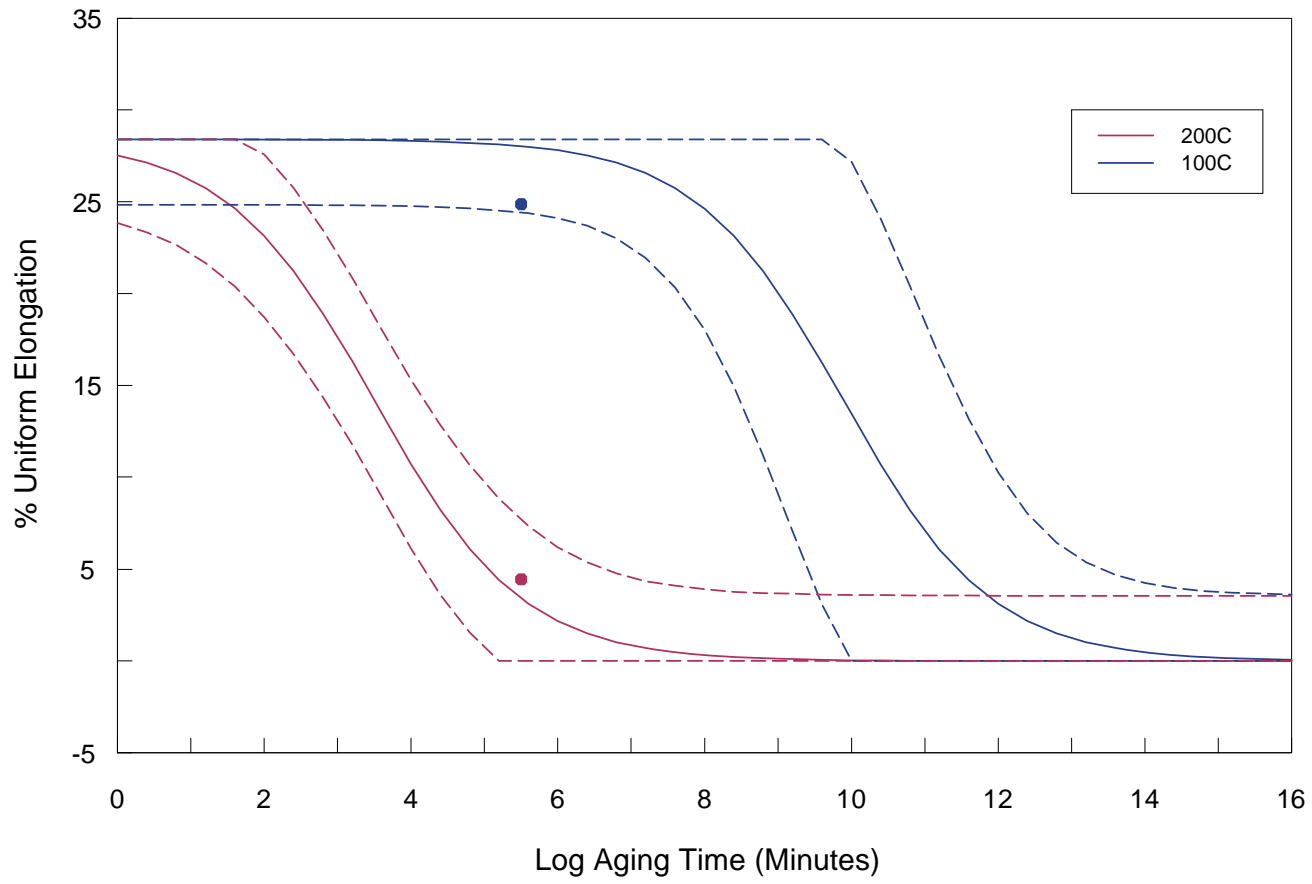

Figure 4.33. U-7.7Nb validation UE data (points) and model predictions (solid lines—mean, dashed lines_-95\% prediction intervals). 


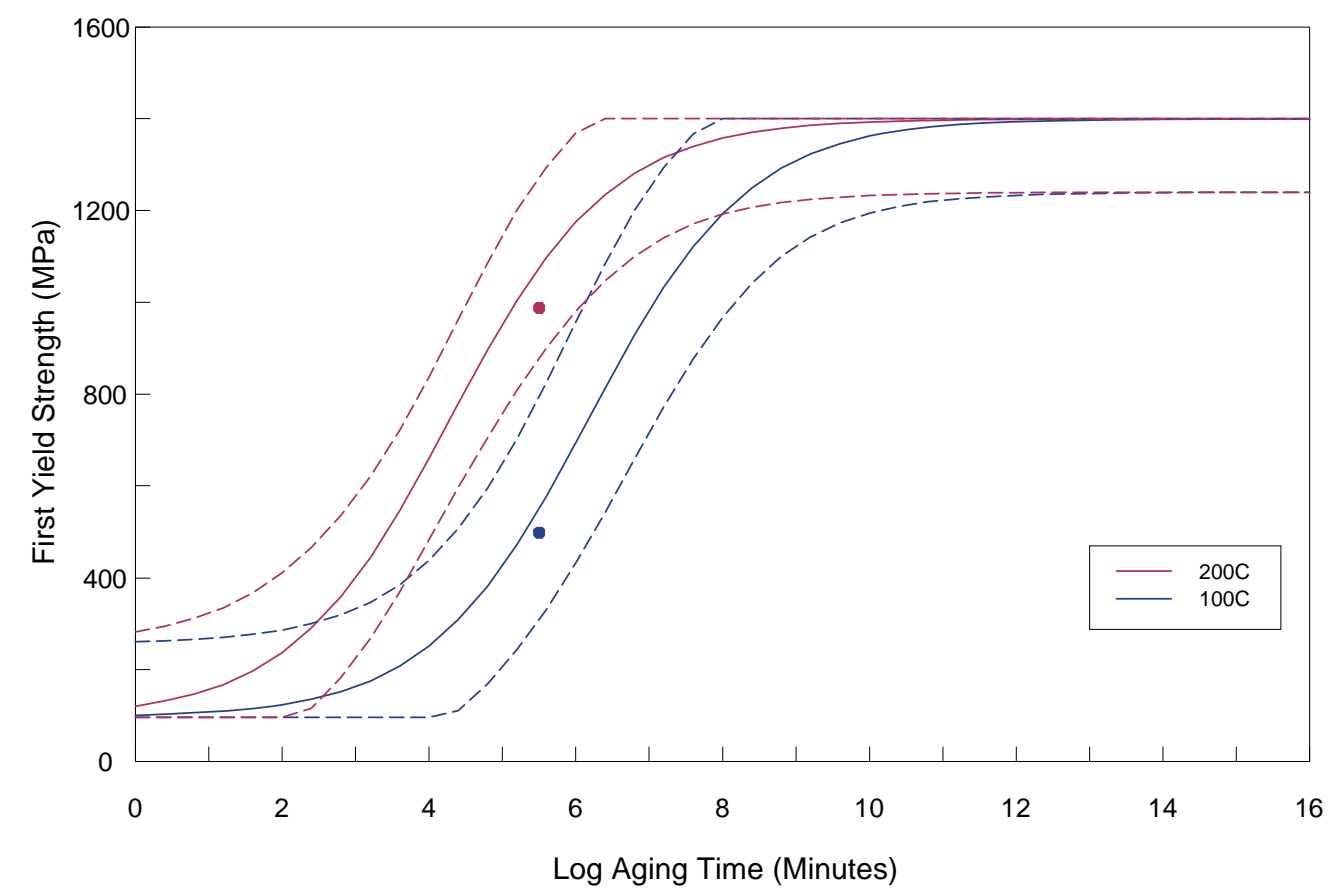

Figure 4.34. U-7.7Nb validation 1YS data (points) and model predictions (solid lines-mean, dashed lines-95\% prediction intervals).

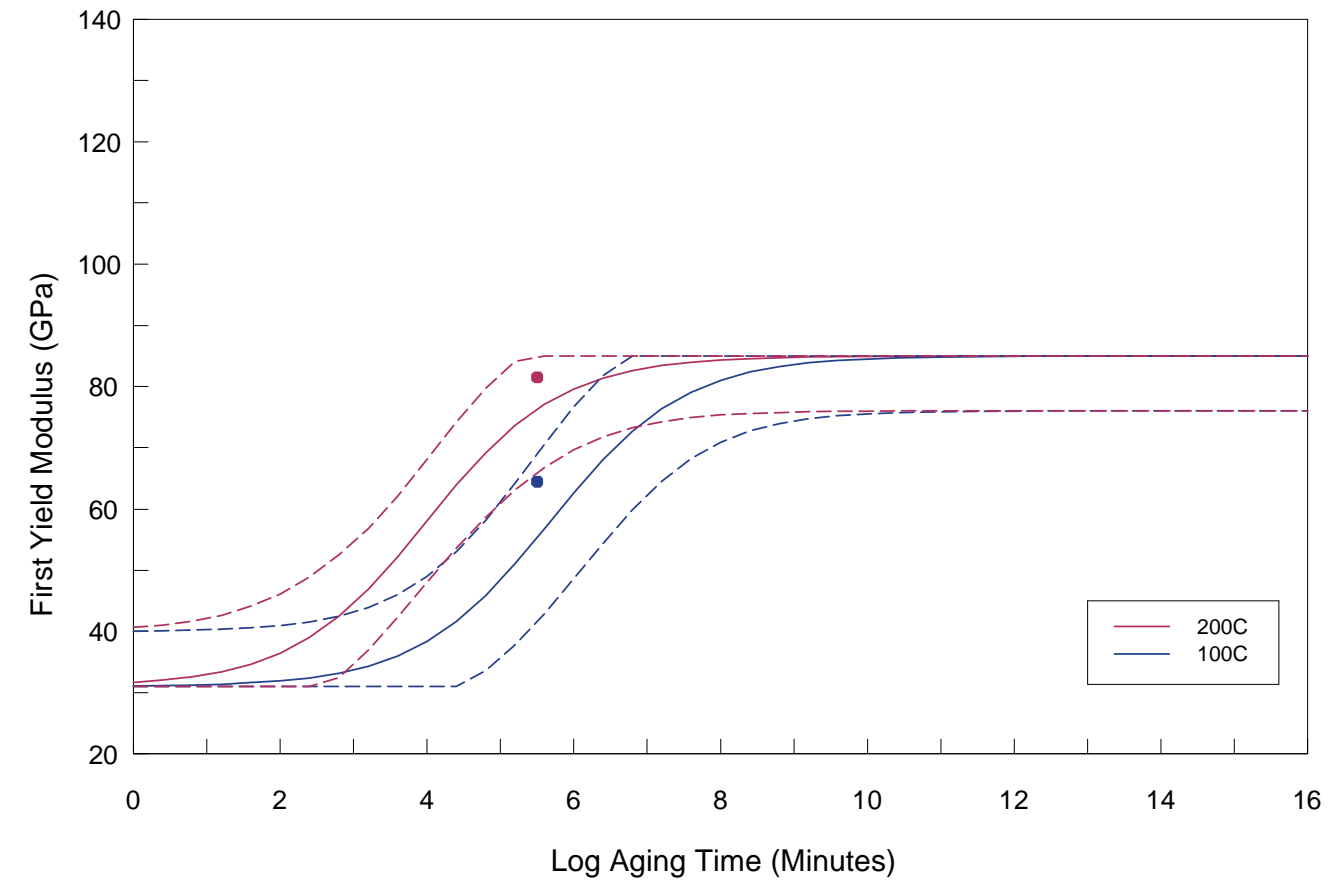

Figure 4.35. U-7.7Nb validation 1YM data (points) and model predictions (solid lines-mean, dashed lines-95\% prediction intervals). 


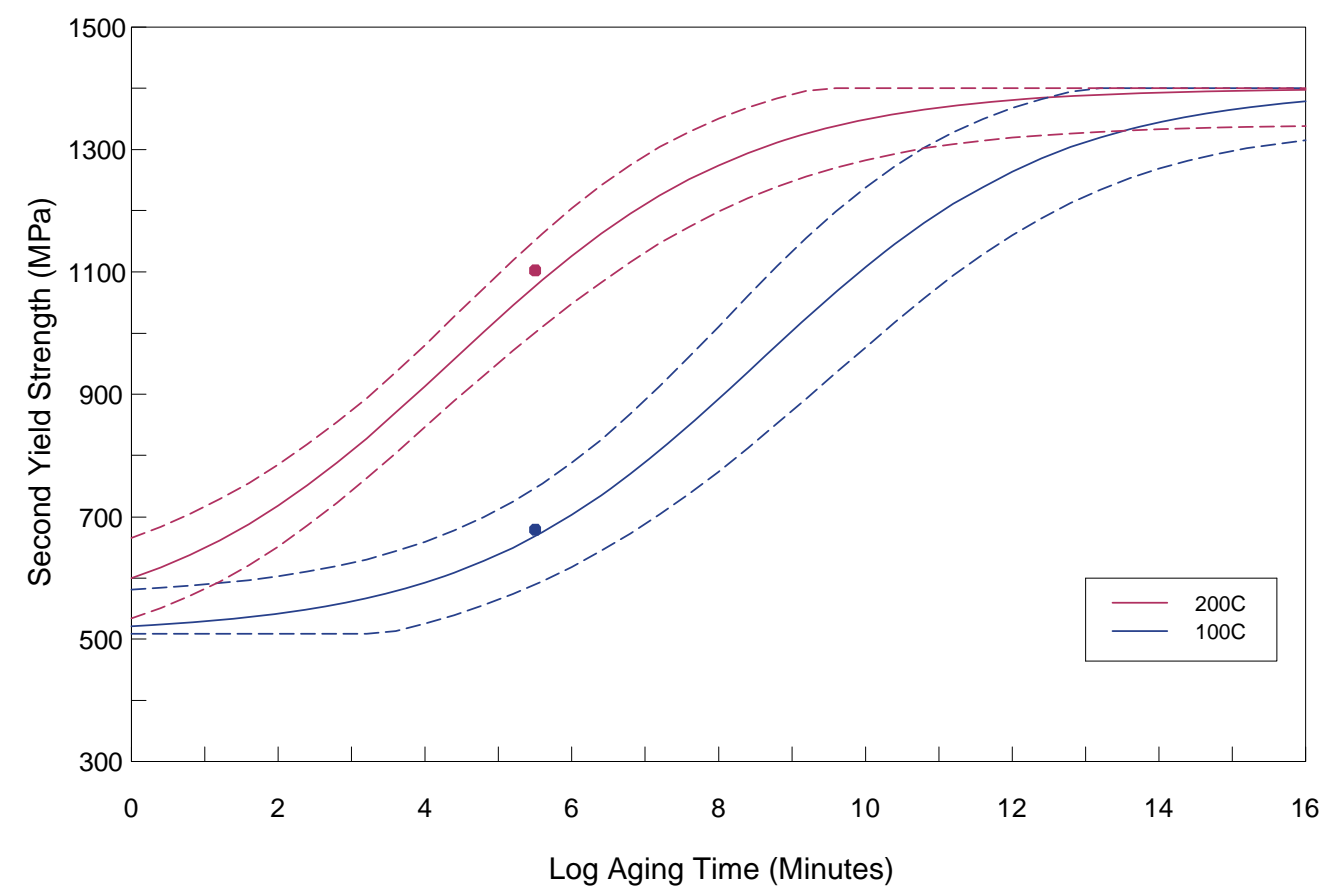

Figure 4.36. U-7.7Nb validation 2YS data (points) and model predictions (solid lines-mean, dashed lines-95\% prediction intervals).

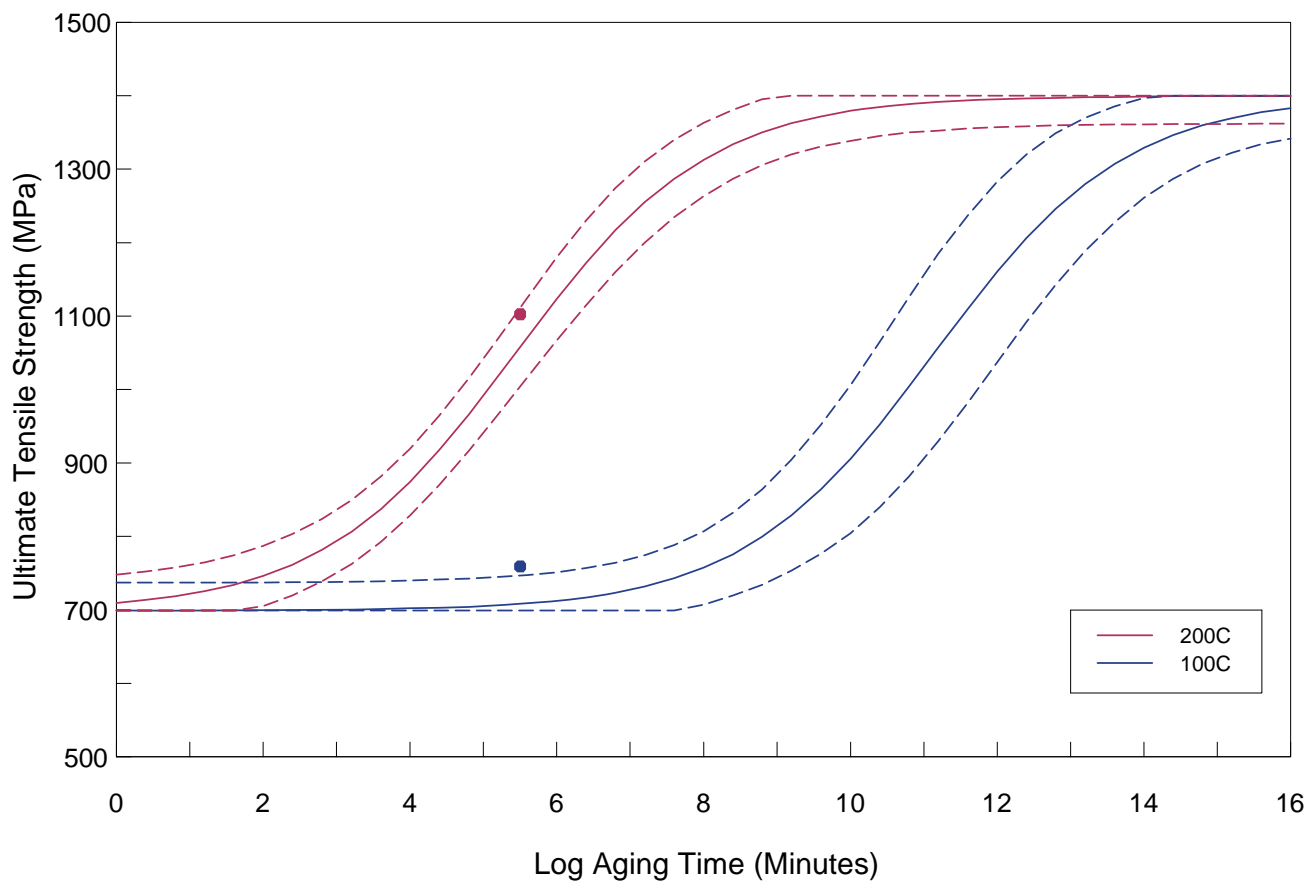

Figure 4.37. U-7.7Nb validation UTS data (points) and model predictions (solid lines—mean, dashed lines_-95\% prediction intervals). 


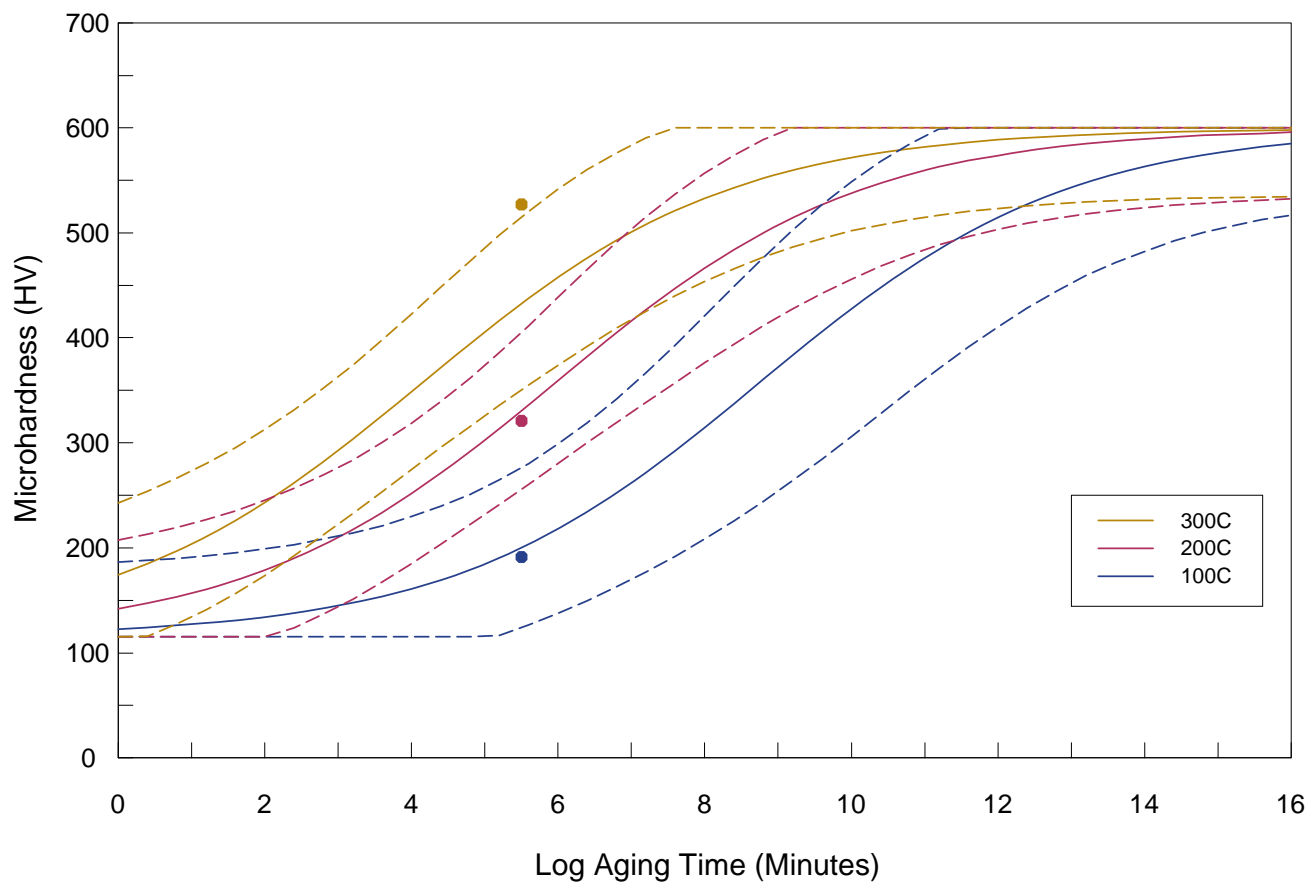

Figure 4.38. U-7.7Nb validation HV data (points) and model predictions (solid lines—mean, dashed lines-95\% prediction intervals). 


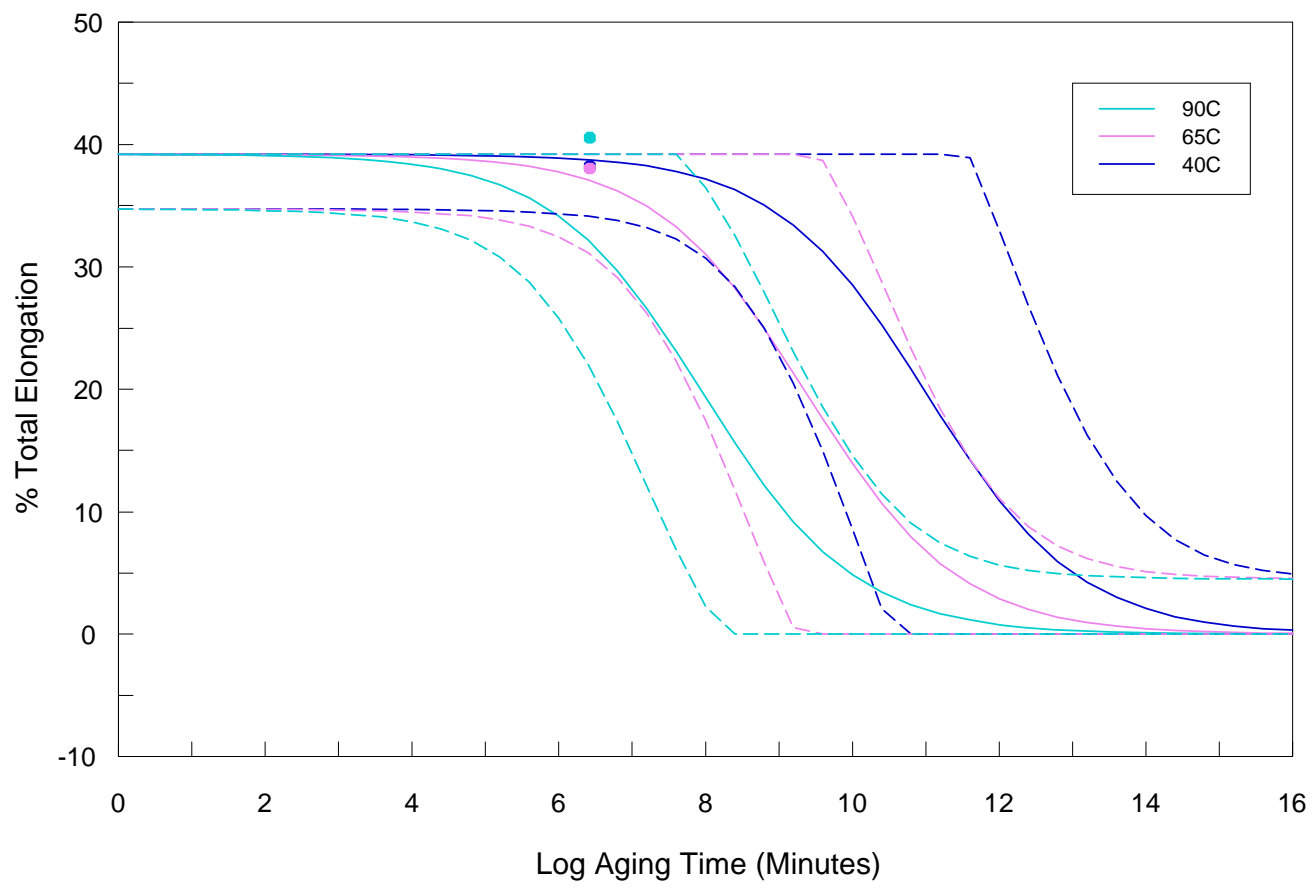

Figure 4.39. U-6Nb validation TE data (points) and model predictions (solid lines-mean, dashed lines-95\% prediction intervals).

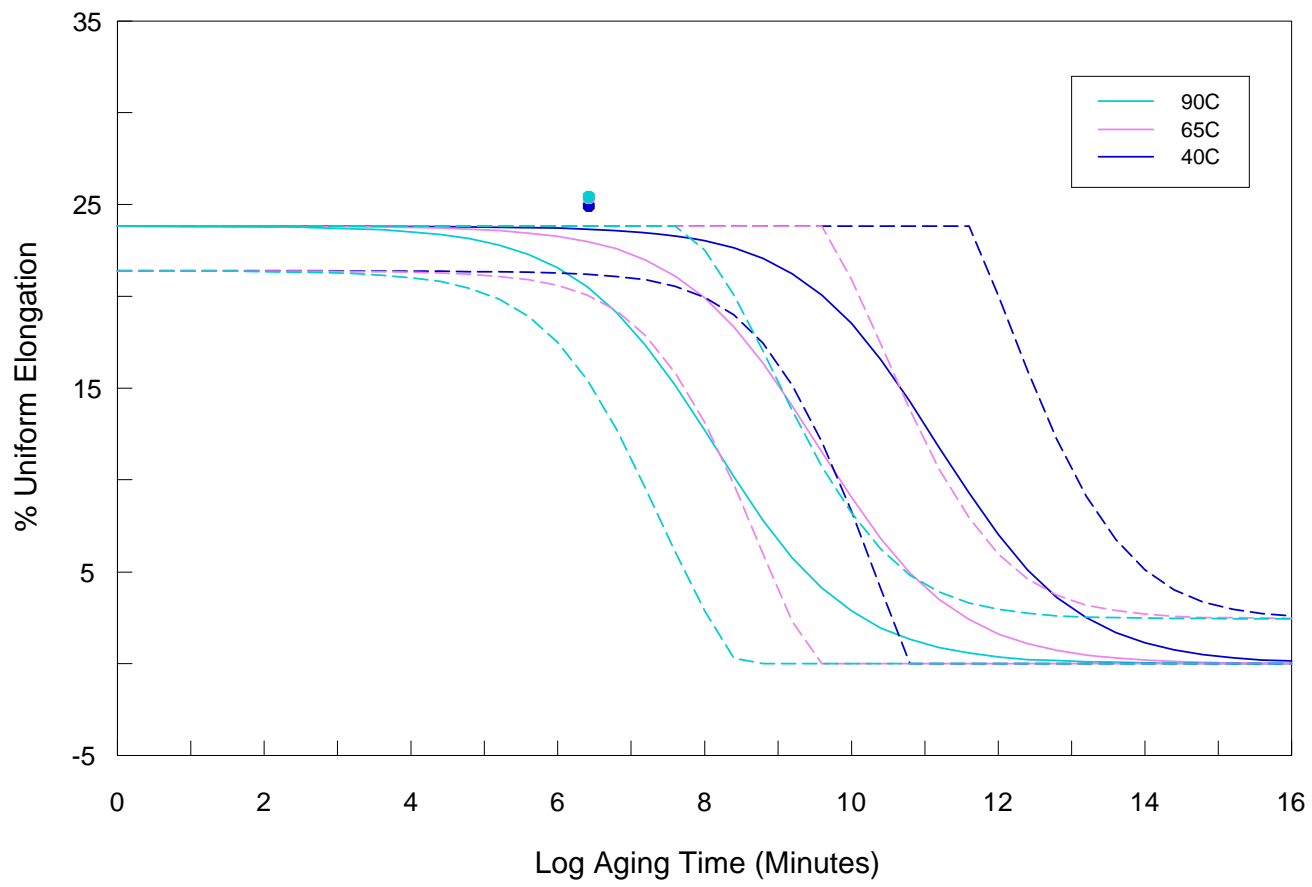

Figure 4.40. U-6Nb validation UE data (points) and model predictions (solid lines-mean, dashed lines-95\% prediction intervals). 


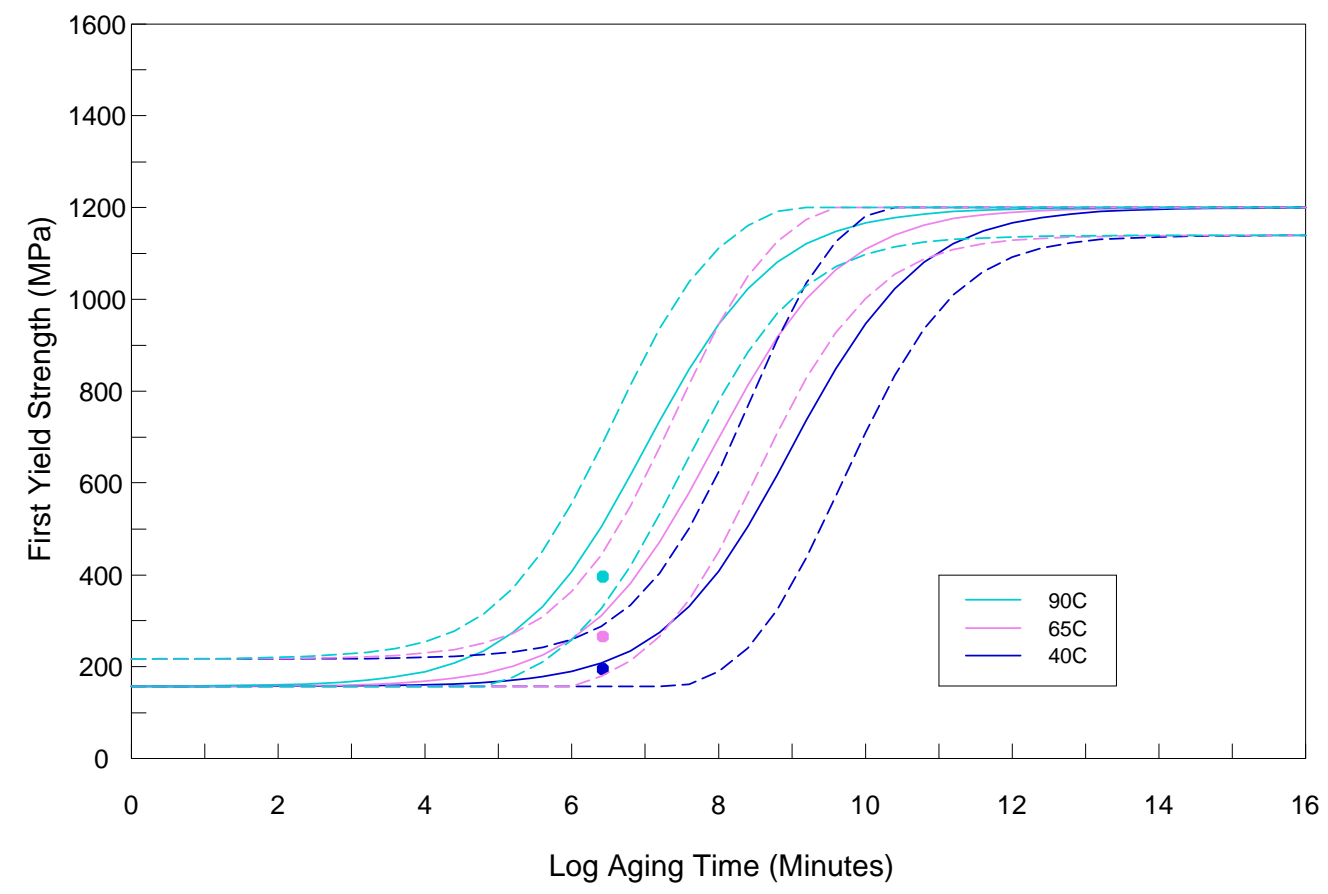

Figure 4.41. U-6Nb validation 1YS data (points) and model predictions (solid lines - mean, dashed lines-95\% prediction intervals.

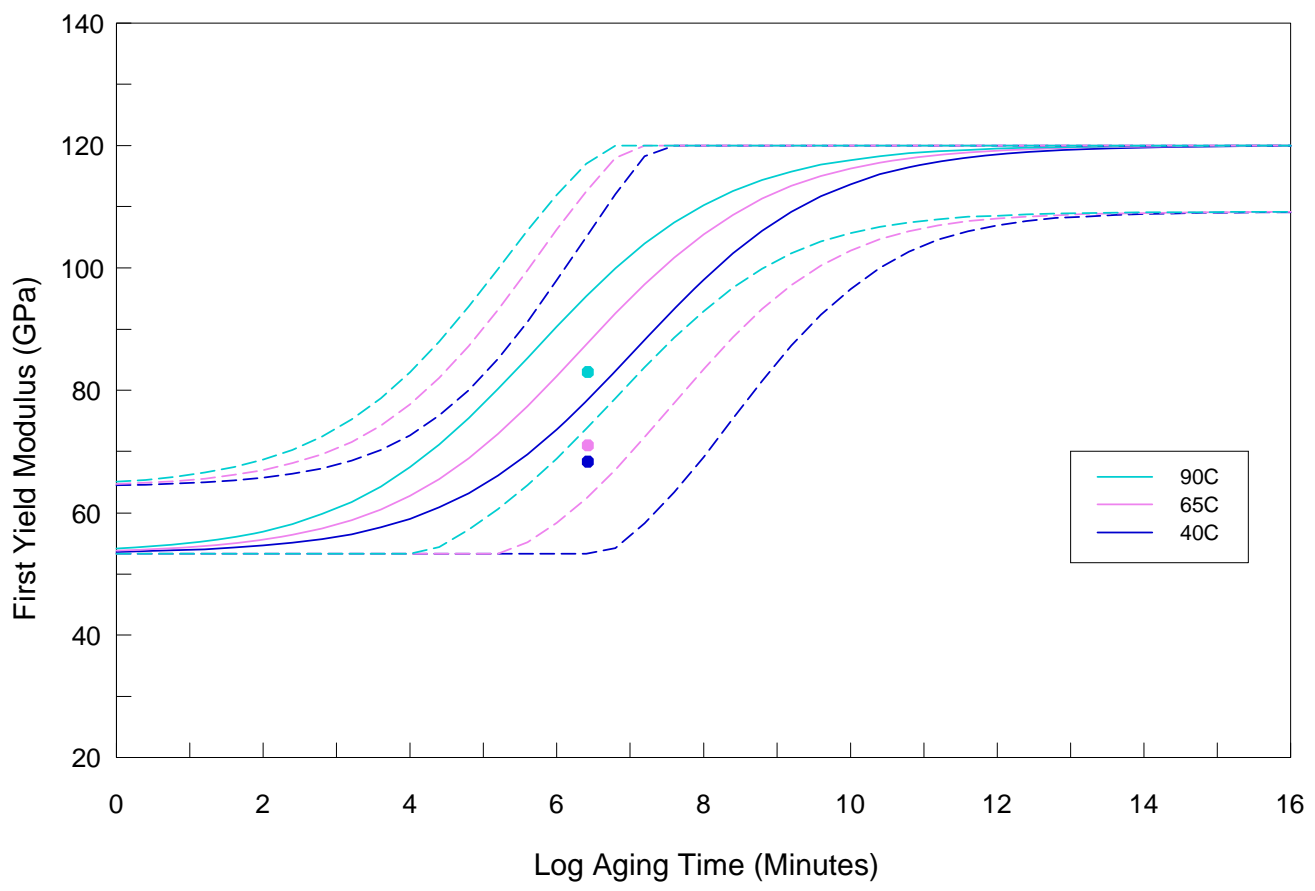

Figure 4.42. U-6Nb validation 1YM data (points) and model predictions (solid lines—mean, dashed lines-95\% prediction intervals. 


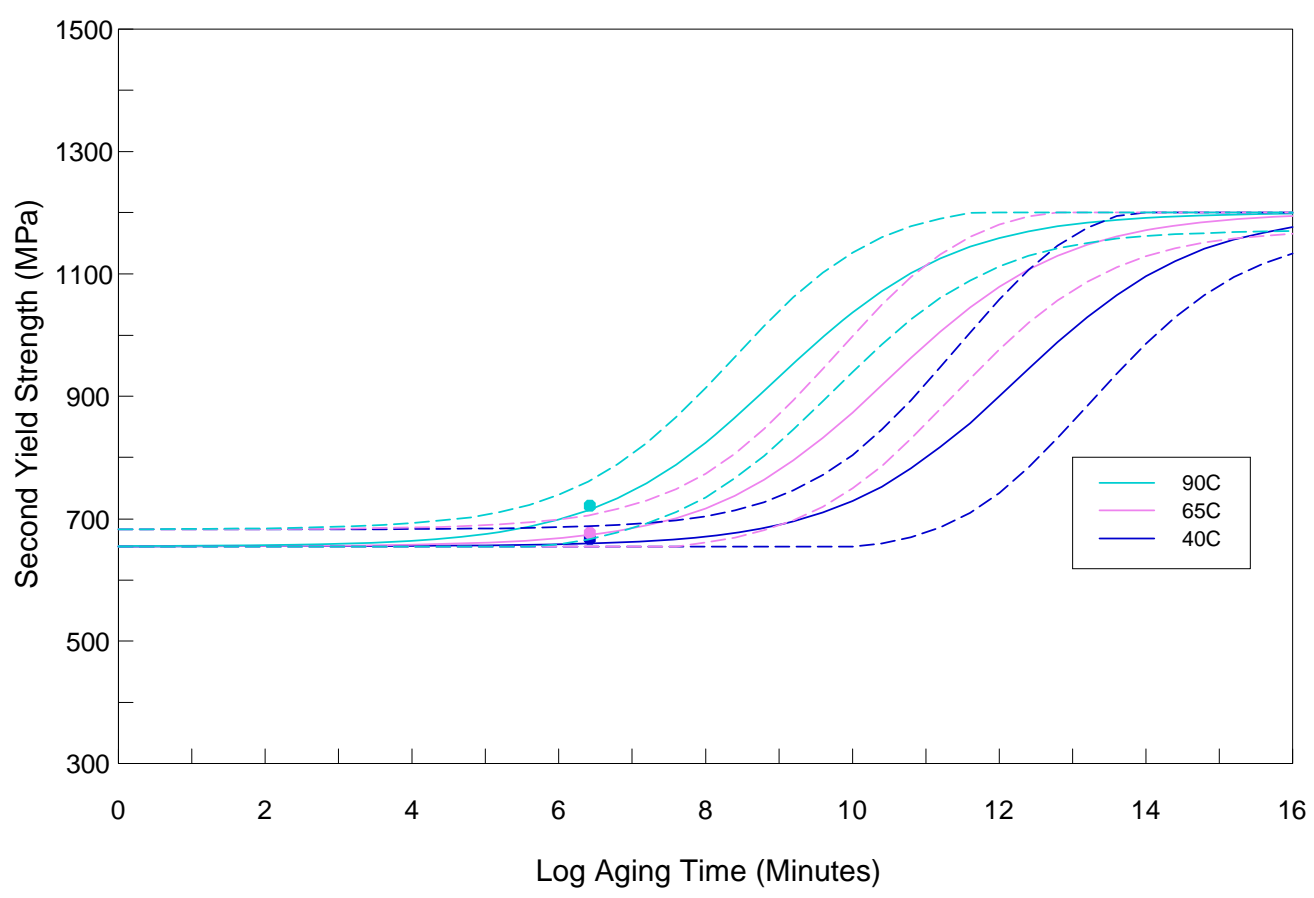

Figure 4.43. U-6Nb validation 2YS data (points) and model predictions (solid lines - mean, dashed lines-95\% prediction intervals).

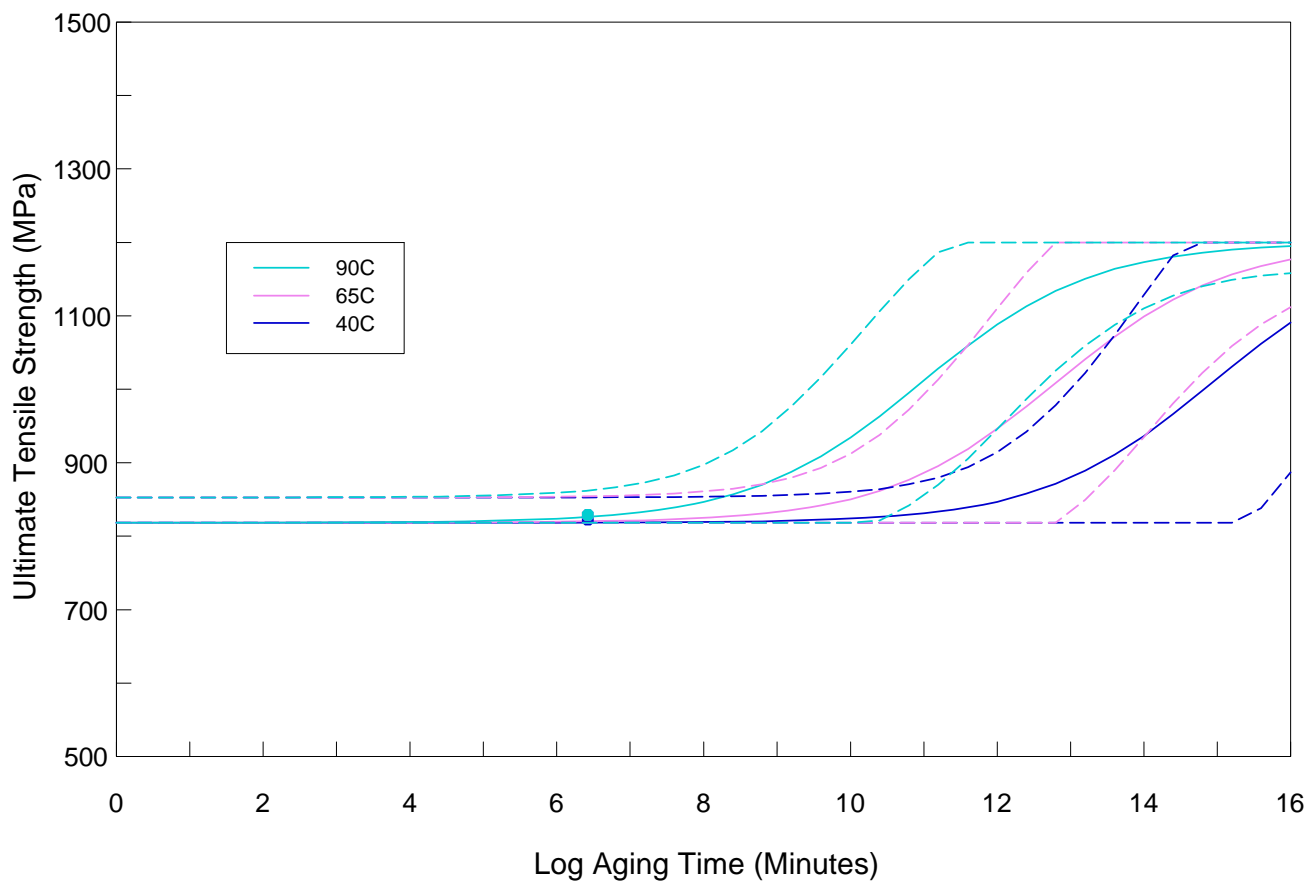

Figure 4.44. U-6Nb validation UTS data (points) and model predictions (solid lines—mean, dashed lines_-95\% prediction intervals). 


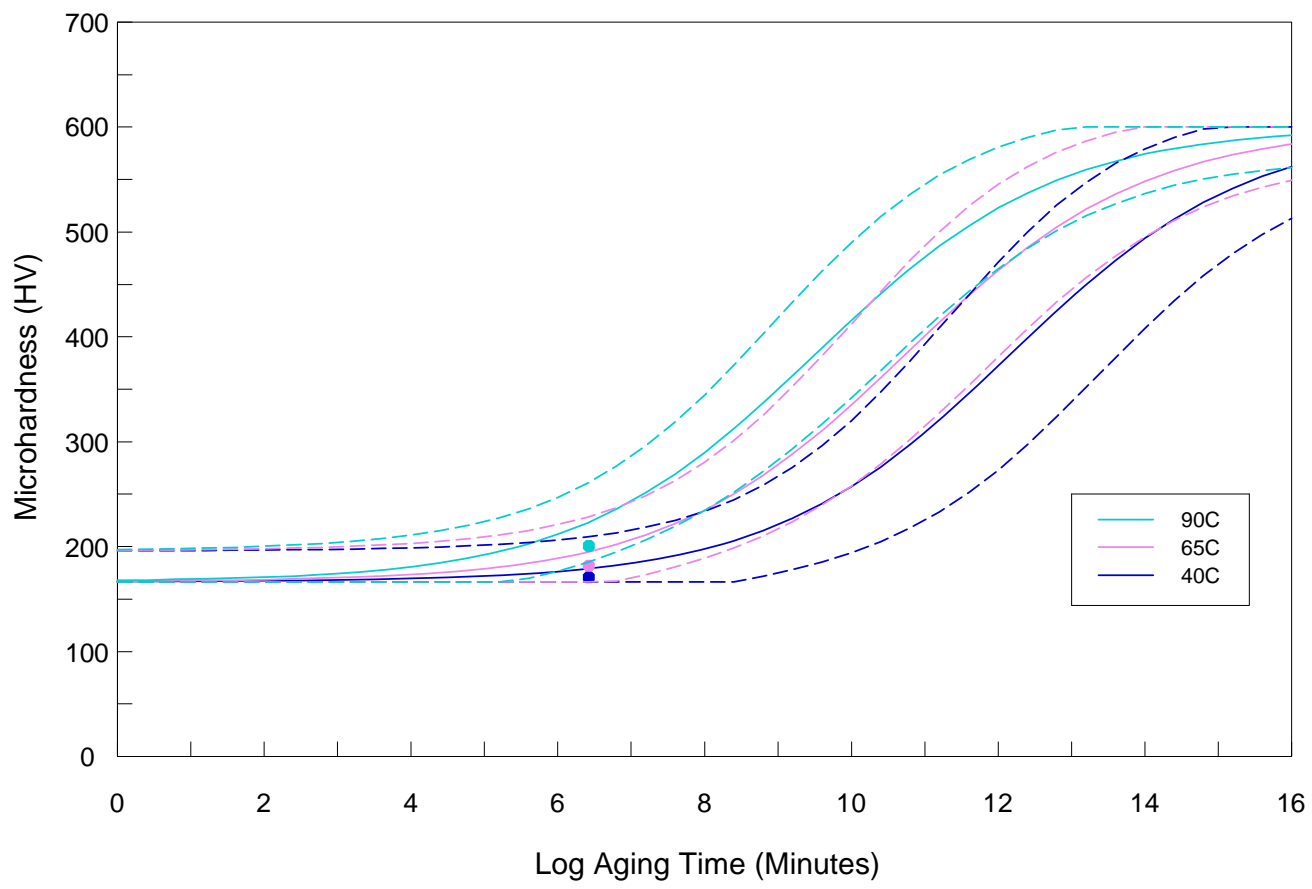

Figure 4.45. U-6Nb validation HV data (points) and model predictions (solid lines-mean, dashed lines-95\% prediction intervals). 


\section{APPENDIX 1: SOURCES AND MAGNITUDES OF SCATTER}

For completeness, the origin and impact of all the known or expected sources of scatter is detailed here. It is grouped by whether the scatter originates from a material-specific issue or from a testing or data-reduction issue.

1. Intrinsic Material Variability. The plate synthesis report provides further details [2007hac1].

a. Chemistry. Differences in Nb content among replicates is expected to be the most significant material-specific contributor to the variability in all age-sensitive properties. Bulk chemical analysis (ICP-MS) on $~ 10$ replicates from the nonbanded plates provided the following average $\pm 1 S D$ wt $\% \mathrm{Nb}$ : $5.60 \pm 0.56$ and $7.69 \pm 0.33$. The average of $\sim 50$ points taken from more localized electron microprobe (EPMA) scans were $5.30 \pm 0.21$ and $7.75 \pm 0.13$. The smaller $S D$ from the localized EPMA scans indicate that the $\mathrm{Nb}$ content is fairly homogeneous within any given replicate, while the larger scatter from the ICP-MS data indicate that Nb differences among different replicates are more significant. The fact that the coring wavelength and the bulk Nb content measured at eight different positions in the as-cast material showed no systematic variation (top-to-bottom or side-to-side in the mold) indicate that the point-to-point $\mathrm{Nb}$ variations were stochastic and not originating in slow heat flow during solidification, which is normally the main driver of chemical inhomogeneities in alloys, including banded U-6Nb [1978sny1, 1978sny2].

b. Microstructure. Property scatter arising from inclusions and crystal distributions are expected to be minor for this material. With few exceptions, the carbo-oxy-nitride inclusions that affect ductility are uniformly distributed throughout the microstructure. Any crystal anisotropy impacting tensile deformation is expected to be minor because the small average prior gamma grain diameter ( 200 microns) and martensite twin width (20-50 nm) are considerably smaller than the tensile gage diameter (2,540 microns), allowing any anisotropy to be averaged out.

c. Specimen correlations. The $\sim 30$ coupon and $\sim 60$ tensile specimens used in this study were machined from predetermined zones within the plate. However, the replicates for any given age were randomly selected from a bag where they were mixed, so no systematic scatter is expected because of correlation between any replicate and its location in the plate before machining with respect to other replicates or ages.

2. Experimental and Data Reduction. Further details are from [2007hac2, 2008ale].

a. Heat treatment Temperatures. Temperatures of the furnace used for $800^{\circ} \mathrm{C}$ or $850^{\circ} \mathrm{C}$ solutionizing and furnaces and sand pot employed for aging were calibrated with an independent thermocouple, ensuring a mean set-point temperature value accurate to within $1^{\circ} \mathrm{C}$. Deviations from this mean value during furnace or sand pot operation are expected to be $\pm 2^{\circ} \mathrm{C}$.

b. Heat treatment times. The impact of the expected scatter in the $800^{\circ} \mathrm{C}$ or $850^{\circ} \mathrm{C}$ solutionizing heat treatment times ( \pm 30 seconds) is considered to be negligible for a 30-minute nominal time. There is an error in the aging process because of the time it took for the vacuum-encapsulated specimens to reach the aging temperature upon heating to the aging temperature, and to reach ambient temperature $\left(23^{\circ} \mathrm{C}\right)$ upon subsequent cooling. All aging heat treatments carried out for times $<1,000$ minutes 
had 10 minutes added to the nominal aging time to account for the time it took to heat up to the aging temperature. (The times quoted here and elsewhere [2007hac2] are the nominal times) This was done on the basis of a preliminary experiment that showed it took 10 minutes for the center of a vacuum-encapsulated DU specimen to attain a temperature within $5^{\circ} \mathrm{C}$ of the set point irrespective of whether the set point was $100^{\circ} \mathrm{C}, 200^{\circ} \mathrm{C}, 250^{\circ} \mathrm{C}$, or $300^{\circ} \mathrm{C}$ [2007hac2]. Table A1.1 lists the absolute errors in aging time attributable to the combined effects of (assumed) exponential heating and cooling to/from the aging temperature for both the $<1,000$-minute ages (for which the extra 10 minutes was added) and the $\geq 1,000$-minute ages (for which no extra time was added). The aging equivalency was numerically evaluated in a spreadsheet using the Arrhenius relationship with the indicated activation energy $Q$. The specimens aged $<1,000$ minutes experienced an 1-2 minute surplus of aging, whereas those aged 1,000 minutes or longer experienced a 7-8 minute deficit of aging. These absolute errors are about ten times larger than the actual operator error in pulling the specimen out of the furnace or sand pot at exactly the prescribed time. Table A1.2 shows that the relative aging time error is largest for the 10 minutes (10-20\%) and 100 minutes (1-2\%), but for all other ages it is $1 \%$ or less. The aging time errors were not accounted for in this study since they are $0.01-1 \%$ in the intermediate- to long-time regimes where the sensitivity to such errors in the time-series fitting process would be the highest because of their higher leveraging

c. Solutionizing and quenching replicate specimens in different encapsulations. All the AQ specimens were solution treated and quenched in the same capsule. Because of their quantity, the specimens meant to be subsequently aged were solutionized and quenched in several different capsules, with no attempt to keep track of which specimens came from which capsule. Given the relative quench-insensitivity of U-Nb alloys ( $\geq 20 \mathrm{~K} / \mathrm{s}$ cooling rates are sufficient for this purpose [1984eck]) and the fact that the operator reported no problems or anomalies with this step in the experiment, the impact of using multiple capsules is considered to be minor.

d. Aging replicate specimens in different encapsulations. Two tensile specimen replicates and a single coupon for hardness were aged in the same encapsulated tube at the outset, ensuring that they experience the same aging treatment. In instances where the first two tensile replicates (labeled \#1 and \#2) for a given age gave significantly different stress-strain responses, one or two additional tensile specimen replicates (labeled \#3 and \#4) were heat treated and tested to provide better statistics. It was often the case that the replicates 3 and 4 showed the same scatter amongst themselves as replicates 1 and 2 showed amongst themselves. Such an outcome indicates that the scatter in replicate results is due to the intrinsic point-to-point variability in the chemistry and/or microstructure of the nonbanded plate material and not due to a defective heat treatment. One might consider that a bias is introduced because only the apparently anomalous ages (not all ages) were subjected to further tensile replicate testing. However, this potential source of bias was not considered further.

e. Hardness testing (HV). The observed $\pm \sim 8 \mathrm{HV} S D$ scatter (Tables 2.2 and 2.4) is a combination of indenter errors (force and size measurement), and the intrinsic chemistry and microstructural variability, and the unavoidable situation where a hard carbo-oxy-nitride inclusion is present just below the surface of indention, which 
skews a small portion of the data points higher than normal. Deconvolution of these error sources is neither practical not necessary.

f. Elastic moduli (1YS, 2YS slopes). The 1YS slope was determined by trial-and-error (with the aid of graphing software) on the engineering stress-engineering strain curves. The analyst eyeballed the best linear fit to the curve near the origin. The analyst's error in determining any given 1YS slope is estimated to be $\pm 2500 \mathrm{MPa} S D$. The 2YS slope ${ }^{11}$ was determined by doing a linear fit to the curve in the region of its inflection, and therefore is subject to less absolute error (estimated at $\pm 250 \mathrm{MPa} S D$ ) than that of the 1YS slope. Both of these are dwarfed by the material variability observed in the actual scatter among replicates (Tables 2.1 and 2.3)

g. Tensile stresses (1YS, 2YS, UTS). The MTS screw-driven tensile testing machines have load cells that were calibrated to an accuracy better than $0.1 \%$ relative, which translates to a measurement error of $1 \mathrm{MPa}$ at a typical 1,000 MPa flow stress observed in U-Nb. The initial cross-sectional area measurement (done with calipers and an optical microscope) has a relative error of $0.04 \%$, which translates to an additional stress measurement error of $0.4 \mathrm{MPa}$ at the 1,000 MPa level. These stress errors are smaller than those because of the analyst's error in determining any given $1 Y S$ slope that propagates through the analysis to give a $1 Y S S D$ of $\pm 5-10 \mathrm{MPa}$. More significantly, all of the foregoing errors pale in view of the intrinsic material variability, which reflected in the wide differences in SD (spanning 1.4-180.3 MPa) as implied from the data in Tables 2.1 and 2.3, and explicitly reported in Tables 6.1 and 6.3 in [2007hac2]. (It is noted that the analyst's errors in measuring 2YS and UTS values for any given stress-strain curve are much smaller than those for measuring 1YS.)

h. Tensile elongations (uniform and total). The strain gages used were calibrated to be within $0.1 \%$ relative, which translates to an engineering strain measurement error of 0.00025 at a typical 0.25 total strain (elongation) to failure. Considering the propagation of the error in determining any given 1YS slope translates to an error in the uniform and total plastic elongation of 0.00025 . The material variability again dominates the observed scatter in uniform and TEs, typically $>0.01$ (Tables 2.1 and 2.3). In line with past metallurgical experience, the UE has a smaller scatter than the $\mathrm{TE}$, because the latter is affected by stochastic phenomenon arising from internal defects and surface damage, but the former is not. The uniform (and nonuniform) elongation did, however, suffer from problems with determining the instability point from Considere's criterion on the true-stress $(\sigma)$-true strain $(\varepsilon)$ curves, as the slope $\mathrm{d} \sigma / \mathrm{d} \varepsilon$ crossed the $\sigma-\varepsilon$ curve multiple times (and had considerable noise as well); it was decided that the highest-strain intersection of these two curves was the true point of instability. This decision was validated by the fact that the engineering stress evaluated at this assumed instability point was always within $1 \mathrm{MPa}$ of the UTS, which is consistent with typical metallic tensile behavior. In any event, this instability issue does not factor into the TE evaluation.

\footnotetext{
${ }^{11}$ Although the 2YS slope is age-sensitive, it was not analyzed in this study but is included in Tables 2.1, 2.3, 2.5,
} and 2.9 for the sake of completeness. 
Table A1.1. Calculated Absolute Error in Aging Time (Evaluated at the Indicated Aging Temperature) due to Aging Attributable to Heating and Cooling that was Unaccounted for in the Nominal Aging Time and Heat Treat Practice.

\begin{tabular}{|c|c|c|c|c|c|c|c|c|c|}
\hline \multirow{3}{*}{\multicolumn{2}{|c|}{$\mathrm{Q}$ (kcal/mole) }} & \multicolumn{8}{|c|}{ Time error, minutes relative to the nominal aging time } \\
\hline & & \multicolumn{4}{|c|}{25} & \multicolumn{4}{|c|}{30} \\
\hline & & 100 & 200 & 250 & 300 & 100 & 200 & 250 & 300 \\
\hline Nominal & $<1000$ & 1.693 & 1.887 & 2.047 & 2.199 & 1.087 & 1.438 & 1.625 & 1.794 \\
\hline aging & $>=1000$ & -7.821 & -7.325 & -7.048 & -6.795 & -8.427 & -7.773 & -7.471 & -7.200 \\
\hline
\end{tabular}

Table A1.2. Calculated Relative Percentage Error in Aging Time (Evaluated at the Indicated Aging Temperature) due to Aging Attributable to Heating and Cooling that was Unaccounted for in the Nominal Aging Time and Heat Treat Practice.

\begin{tabular}{|c|c|c|c|c|c|c|c|c|c|}
\hline \multirow{2}{*}{\multicolumn{2}{|c|}{ Q reairitio }} & \multicolumn{8}{|c|}{ Time error, percent relative to the nominal aging time } \\
\hline & & \multicolumn{4}{|c|}{25} & \multicolumn{4}{|c|}{30} \\
\hline Aging tem & rature $(\mathrm{C})$ & 100 & 200 & 250 & 300 & 100 & 200 & 250 & 300 \\
\hline \multirow{11}{*}{$\begin{array}{l}\text { Nominal } \\
\text { aging } \\
\text { time (min) }\end{array}$} & 10 & 16.9300 & 18.8700 & 20.4700 & 21.9900 & 10.8700 & 14.3800 & 16.2500 & 17.9400 \\
\hline & 100 & 1.6930 & 1.8870 & 2.0470 & 2.1990 & 1.0870 & 1.4380 & 1.6250 & 1.7940 \\
\hline & 165 & 1.0261 & 1.1436 & 1.2406 & 1.3327 & 0.6588 & 0.8715 & 0.9848 & 1.0873 \\
\hline & 215 & 0.7874 & 0.8777 & 0.9521 & 1.0228 & 0.5056 & 0.6688 & 0.7558 & 0.8344 \\
\hline & 1000 & -0.7821 & -0.7325 & \begin{tabular}{|c|}
-0.7048 \\
\end{tabular} & -0.6795 & -0.8427 & -0.7773 & -0.7471 & -0.7200 \\
\hline & 10000 & -0.0782 & -0.0733 & -0.0705 & -0.0680 & -0.0843 & -0.0777 & -0.0747 & -0.0720 \\
\hline & 100000 & -0.0078 & -0.0073 & -0.0070 & -0.0068 & -0.0084 & -0.0078 & -0.0075 & -0.0072 \\
\hline & 230385 & -0.0034 & -0.0032 & -0.0031 & -0.0029 & -0.0037 & -0.0034 & -0.0032 & -0.0031 \\
\hline & 318000 & -0.0025 & -0.0023 & -0.0022 & -0.0021 & -0.0027 & -0.0024 & -0.0023 & -0.0023 \\
\hline & 1052400 & -0.0007 & -0.0007 & -0.0007 & -0.0006 & -0.0008 & -0.0007 & -0.0007 & -0.0007 \\
\hline & 3153600 & -0.0002 & -0.0002 & -0.0002 & -0.0002 & -0.0003 & -0.0002 & -0.0002 & -0.0002 \\
\hline
\end{tabular}




\section{APPENDIX 2: MODEL FITS AND PREDICTIONS ON AN ALTERNATE DEFINITION OF HARDNESS REPLICATES}

For completeness, the current analysis method was repeated on the hardness data, using a different assumption on the definition of what constitutes a hardness replicate. The approach documented in the main text assumed that the hardness replicate was the average of all measurements from a single coupon. For all the model-fitting and validation data in this work, the number of hardness replicates, $n$ or $n v$ for any given age, is always equal to one. This Appendix presents the parallel results assuming that the hardness replicate is the individual hardness measurement. These replicate hardness measurements are provided in Tables 2.2 and 2.4 for the U-5.6Nb and U-7.7Nb model-fitting data ( $n$ varying over $7-15$ ), and also for the validation HV measurements (Tables 2.6, 2.7, and 2.10, where $n$ is even larger).

The parameters fitted on this alternate assumption are provided in Tables A2.1 and A2.2, which can be compared with the results of the baseline approach in Tables 3.18 and 3.19. The graphical depictions in Figures A2.1 and A2.2 can also be compared against Figures 4.15 and 4.16. The difference between the parameters is minimal, showing that the model fitting is not very sensitive to the replicate definition.

What is sensitive to the definition are the prediction intervals for the validation data, provided in Table A2.3. Compare this with the results from the baseline approach in Table 4.13. Note that the $P I$ values in Table A2.3 are practically indistinguishable from the corresponding CI values. This is because as $n$ increases, the quantity RSE/ $n$ approaches zero, and the expression for $P I$ (Eq. A4.13) approaches that for the CI (Eq. A4.12); See Appendix 4. The sample size for future ages was set at a comparable number to the validation hardness data sets already available: $n v=160$ for $\mathrm{U}-6 \mathrm{Nb}$ and $n v=60$ for both U-5.6Nb and U-7.7Nb.

In general, the baseline approach (Table 4.13) gives better agreement between the existing validation data and the model predictions than does this alternate approach (Table A2.3), which gives some modest confidence in the baseline approach. 
Table A2.1. Vickers Hardness Kinetic Parameters for U-5.6Nb. The $t$ value used to compute the confidence interval is 1.969 . The units of $Q$ are $\mathrm{kcal} / \mathrm{mol} ; A$ and $B$ were assessed on the basis of the time in minutes.

\begin{tabular}{|l|r|r|r|r|}
\hline \multicolumn{5}{|c|}{ U-5.6Nb Microhardness } \\
\hline Parameter & Estimate & Standard Error & \multicolumn{2}{|c|}{ 95\% Confidence Interval } \\
\cline { 4 - 5 } & & & Lower Bound & \multicolumn{1}{c|}{ Upper Bound } \\
\hline$Q$ & 27.575 & 0.729 & 26.140 & 29.010 \\
\hline$A$ & 0.6148 & 0.0153 & 0.5847 & 0.6449 \\
\hline$B$ & 3.4009 & 0.0361 & 3.3298 & 3.4720 \\
\hline
\end{tabular}

Table A2.2. Vickers Hardness Kinetic Parameters for U-7.7Nb. The $t$ value used to compute the confidence interval is 1.976 . The units of $Q$ are $\mathrm{kcal} / \mathrm{mol} ; A$ and $B$ were assessed on the basis of the time in minutes.

\begin{tabular}{|l|r|r|r|r|}
\hline \multicolumn{5}{|c|}{ U-7.7Nb Microhardness } \\
\hline Parameter & Estimate & Standard Error & \multicolumn{2}{|c|}{ 95\% Confidence Interval } \\
\cline { 4 - 5 } & & & \multicolumn{1}{c|}{ Lower Bound } & \multicolumn{1}{c|}{ Upper Bound } \\
\hline$Q$ & 21.814 & 1.263 & 19.318 & 24.310 \\
\hline$A$ & 0.4555 & 0.0228 & 0.4104 & 0.5006 \\
\hline$B$ & 4.2665 & 0.1117 & 4.0458 & 4.4872 \\
\hline
\end{tabular}


Table A2.3. Experimentally Measured and Predicted and Mean HV Values for Various U-Nb Alloys for Current and Future Model Validation, from an Alternate Replicate Definition. The $95 \%$ prediction and confidence intervals are also given. The alloy identified as $6 \mathrm{wt} \% \mathrm{Nb}$ is the RFP banded $\mathrm{U}-6 \mathrm{Nb}$; the alloys identified as $5.6 \mathrm{wt} \%$ and $7.7 \mathrm{wt} \%$ are nonbanded. "NP" indicates that no data point is expected, because of material limitations.

\begin{tabular}{|c|c|c|c|c|c|c|c|c|c|}
\hline \multirow{3}{*}{$\begin{array}{l}\text { Alloy } \\
\text { Wt.\% } \\
\text { Nb }\end{array}$} & \multirow{3}{*}{$\begin{array}{l}\text { Aging } \\
\text { Temp } \\
\text { (C) }\end{array}$} & \multirow{2}{*}{\multicolumn{2}{|c|}{$\begin{array}{l}\text { Aging } \\
\text { Time }\end{array}$}} & \multicolumn{6}{|c|}{ Vickers Microhardness } \\
\hline & & & & \multirow{2}{*}{\begin{tabular}{|l} 
Experimental \\
Mean
\end{tabular}} & \multicolumn{2}{|c|}{ Prediction Int. } & \multirow{2}{*}{\begin{tabular}{|l|} 
Prediction \\
Mean
\end{tabular}} & \multicolumn{2}{|c|}{ Confidence Int. } \\
\hline & & $(\min )$. & (years) & & lower & upper & & lower & upper \\
\hline 6 & 40 & 328320 & 0.625 & & 172.5 & 175.7 & 174.1 & 172.5 & 175.6 \\
\hline 6 & 40 & 656640 & 1.25 & & 173.6 & 177.4 & 175.5 & 173.6 & 177.4 \\
\hline 6 & 40 & 1311840 & 2.5 & & 175.0 & 179.5 & 177.3 & 175.0 & 179.5 \\
\hline 6 & 40 & 2629440 & 5.0 & 171 & 176.7 & 182.0 & 179.4 & 176.7 & 182.0 \\
\hline 6 & 40 & 5260320 & 10.0 & & 178.7 & 185.0 & 181.8 & 178.7 & 185.0 \\
\hline 6 & 40 & 10519200 & 20.0 & & 181.0 & 188.5 & 184.8 & 181.1 & 188.5 \\
\hline 6 & 40 & 21038400 & 40.0 & & 183.8 & 192.7 & 188.3 & 183.9 & 192.7 \\
\hline 6 & 65 & 328320 & 0.625 & & 181.0 & 186.9 & 183.9 & 181.0 & 186.9 \\
\hline 6 & 65 & 656640 & 1.25 & & 183.8 & 190.7 & 187.2 & 183.8 & 190.7 \\
\hline 6 & 65 & 1311840 & 2.5 & & 187.0 & 195.3 & 191.2 & 187.1 & 195.3 \\
\hline 6 & 65 & 2629440 & 5.0 & 182 & 191.0 & 200.6 & 195.8 & 191.0 & 200.6 \\
\hline 6 & 65 & 5260320 & 10.0 & & 195.6 & 206.9 & 201.3 & 195.6 & 206.9 \\
\hline 6 & 65 & 10519200 & 20.0 & & 201.2 & 214.3 & 207.7 & 201.0 & 214.3 \\
\hline 6 & 65 & 21038400 & 40.0 & & 207.3 & 222.8 & 215.0 & 207.3 & 222.8 \\
\hline 6 & 90 & 328320 & 0.625 & & 197.1 & 206.3 & 201.7 & 197.1 & 206.3 \\
\hline 6 & 90 & 656640 & 1.25 & & 202.7 & 213.5 & 208.1 & 202.7 & 213.5 \\
\hline 6 & 90 & 1311840 & 2.5 & & 209.3 & 221.8 & 215.6 & 209.3 & 221.8 \\
\hline 6 & 90 & 2629440 & 5.0 & 201 & 217.0 & 231.5 & 224.2 & 217.0 & 231.5 \\
\hline 6 & 90 & 5260320 & 10.0 & & 225.7 & 242.5 & 234.1 & 225.7 & 242.5 \\
\hline 6 & 90 & 10519200 & 20.0 & & 235.7 & 254.9 & 245.3 & 235.7 & 254.9 \\
\hline 6 & 90 & 21038400 & 40.0 & & 247.0 & 268.9 & 257.9 & 247.0 & 268.9 \\
\hline & & & & & & & & & \\
\hline 5.6 & 100 & 318000 & 0.6 & 189 & 188.2 & 199.2 & 193.7 & 188.2 & 199.2 \\
\hline 5.6 & 100 & 1052400 & 2.0 & & 201.7 & 215.9 & 208.8 & 201.7 & 215.9 \\
\hline 5.6 & 100 & 3153600 & 6.0 & & 217.3 & 235.0 & 226.1 & 217.3 & 235.0 \\
\hline 5.6 & 200 & 318000 & 0.6 & 367 & 357.8 & 373.0 & 365.4 & 357.9 & 373.0 \\
\hline 5.6 & 200 & 1052400 & 2.0 & & 392.6 & 410.3 & 401.5 & 392.7 & 410.3 \\
\hline 5.6 & 200 & 3153600 & 6.0 & & 423.7 & 442.9 & 433.3 & 423.7 & 442.9 \\
\hline 5.6 & 300 & 318000 & 0.6 & 530 & 495.5 & 509.3 & 502.4 & 495.6 & 509.3 \\
\hline 5.6 & 300 & 1052400 & 2.0 & & 518.1 & 531.2 & 524.7 & 518.2 & 531.2 \\
\hline 5.6 & 300 & 3153600 & 6.0 & & 535.3 & 547.3 & 541.3 & 535.4 & 547.3 \\
\hline & & & & & & & & & \\
\hline 7.7 & 100 & 318000 & 0.6 & 191 & 194.3 & 218.1 & 206.2 & 194.3 & 218.1 \\
\hline 7.7 & 100 & 1052400 & 2.0 & & 211.0 & 239.1 & 225.0 & 211.0 & 239.0 \\
\hline 7.7 & 100 & 3153600 & 6.0 & & 228.2 & 260.8 & 244.5 & 228.3 & 260.8 \\
\hline 7.7 & 200 & 318000 & 0.6 & 321 & 317.3 & 340.8 & 329.0 & 317.3 & 340.8 \\
\hline 7.7 & 200 & 1052400 & 2.0 & & 343.4 & 371.8 & 357.6 & 343.4 & 371.8 \\
\hline 7.7 & 200 & 3153600 & 6.0 & & 367.5 & 400.1 & 383.8 & 367.5 & 400.1 \\
\hline 7.7 & 300 & 318000 & 0.6 & 527 & 408.3 & 439.9 & 424.1 & 408.3 & 439.8 \\
\hline 7.7 & 300 & 1052400 & 2.0 & & 433.0 & 466.3 & 449.7 & 433.0 & 466.3 \\
\hline 7.7 & 300 & 3153600 & 6.0 & & 454.2 & 488.2 & 471.2 & 454.3 & 488.1 \\
\hline
\end{tabular}




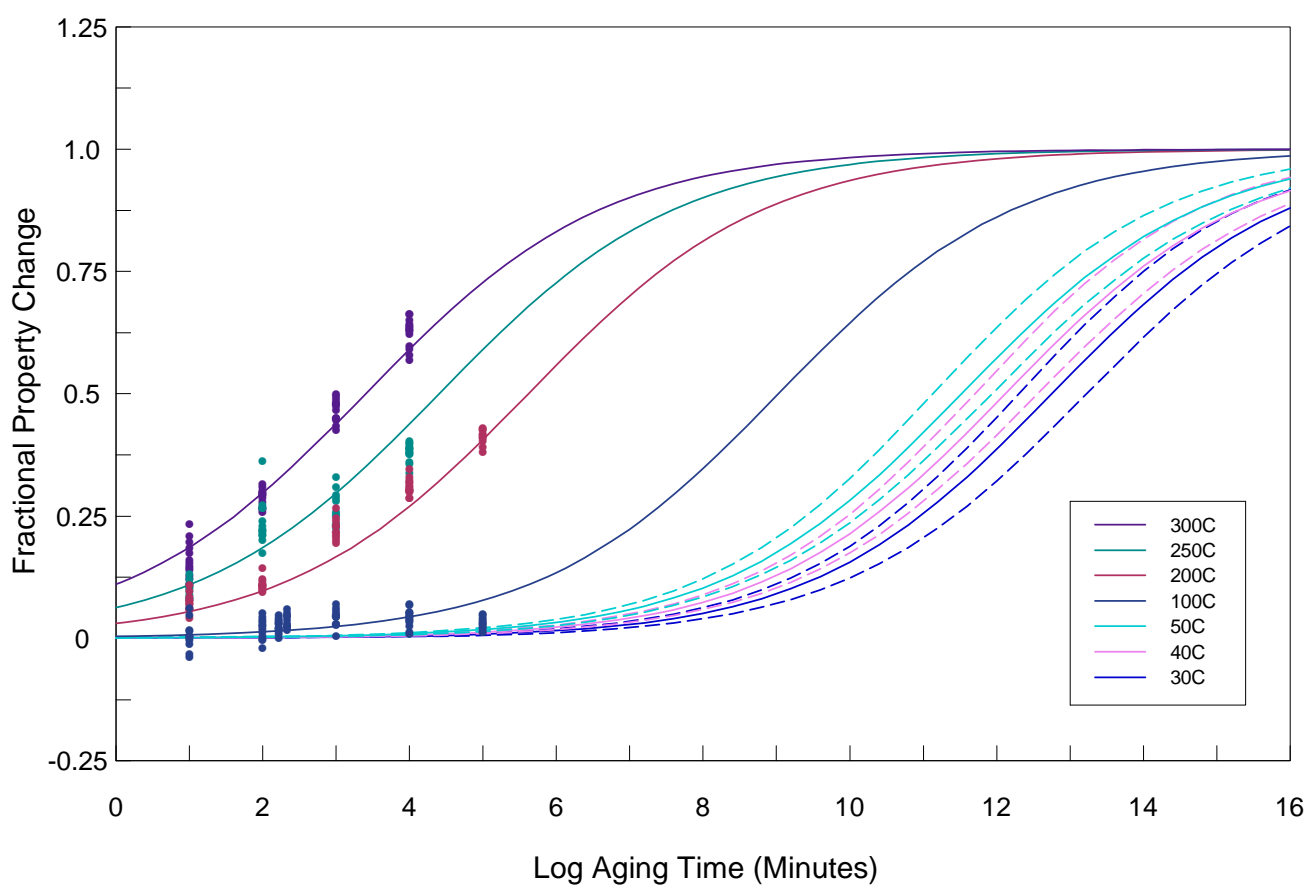

(a)

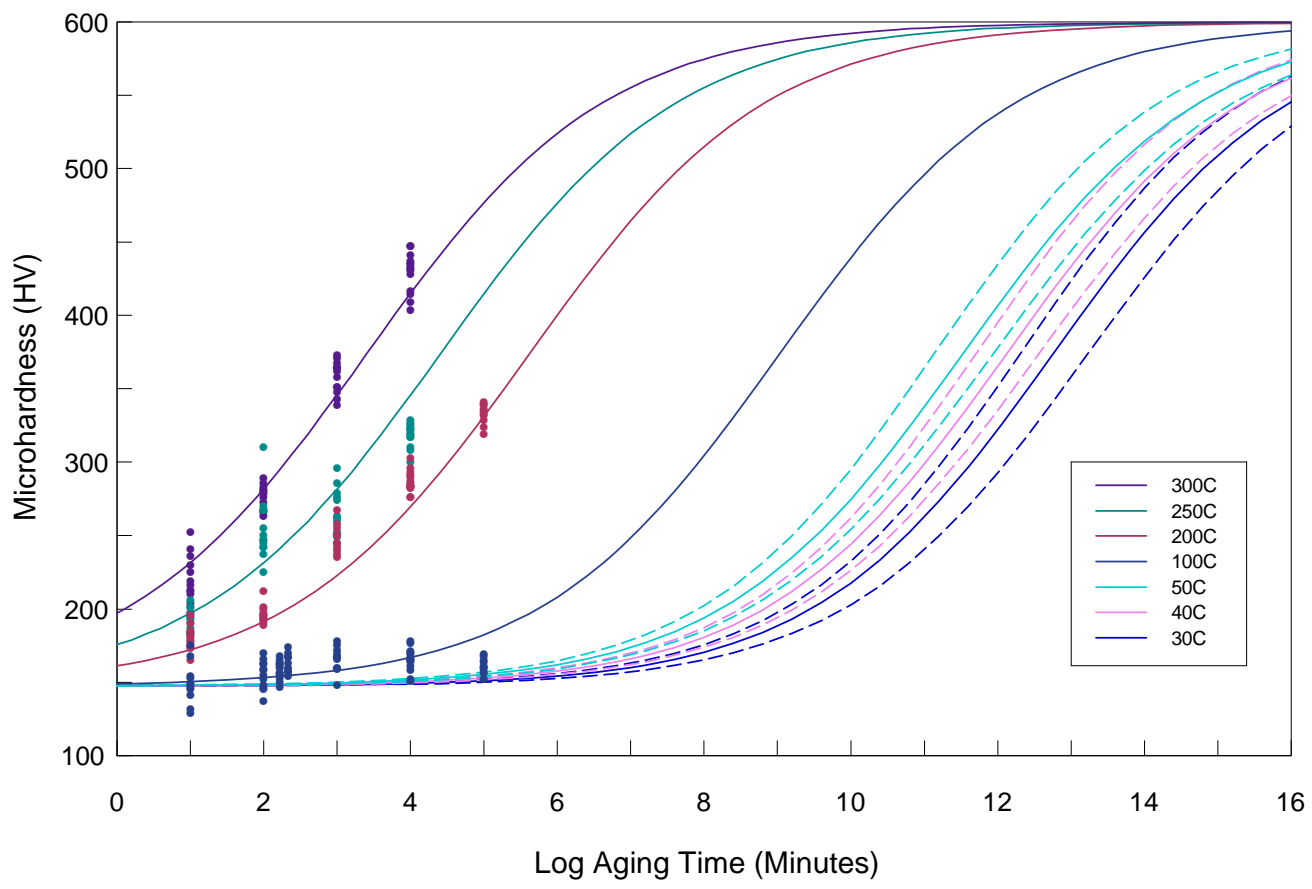

(b)

Figure A2.1. U-5.6Nb HV data (points), model fits to data (solid lines), and low-temperature model predictions (solid line-mean, dashed lines—95\% confidence intervals). (a) in terms of $f$, (b) in terms of absolute property. 


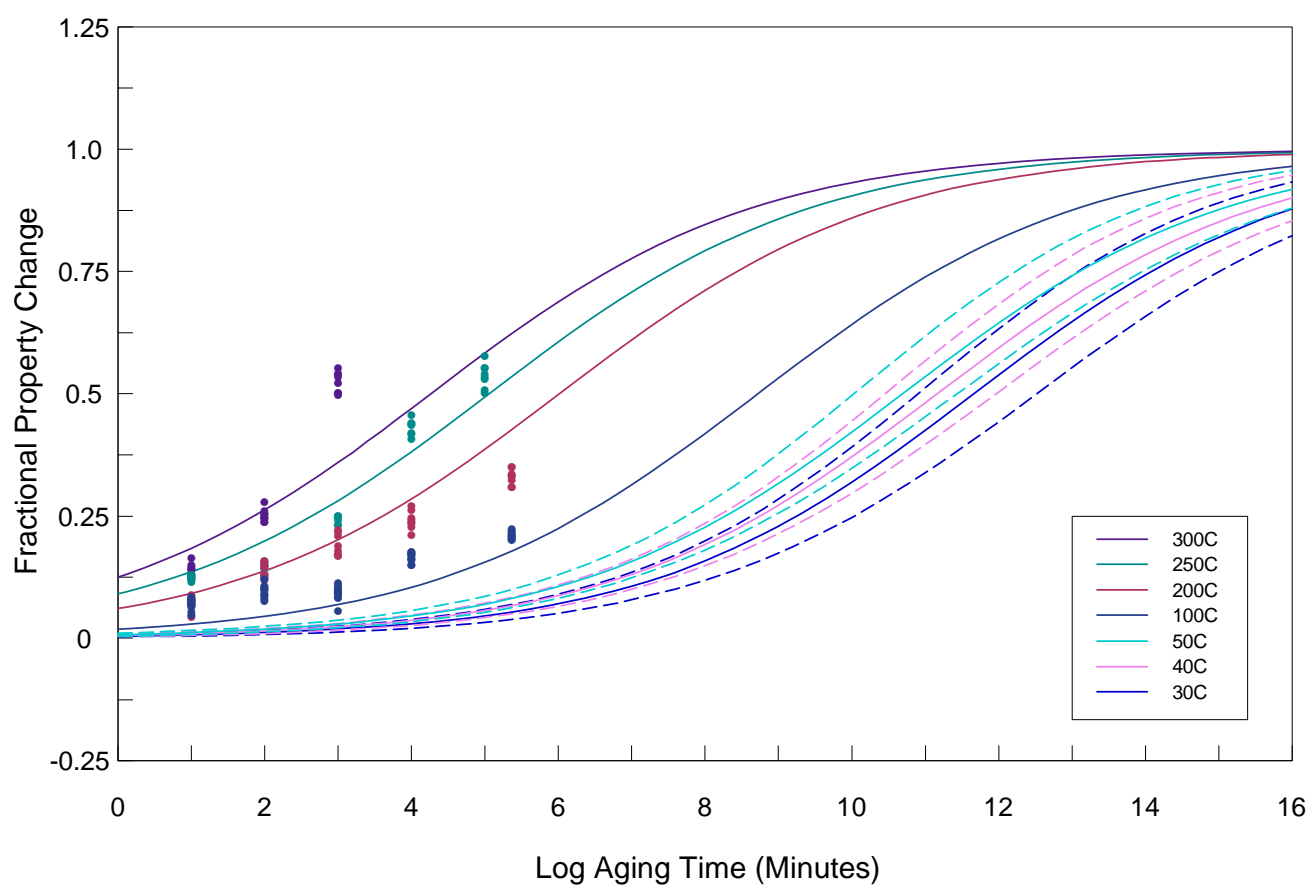

(a)

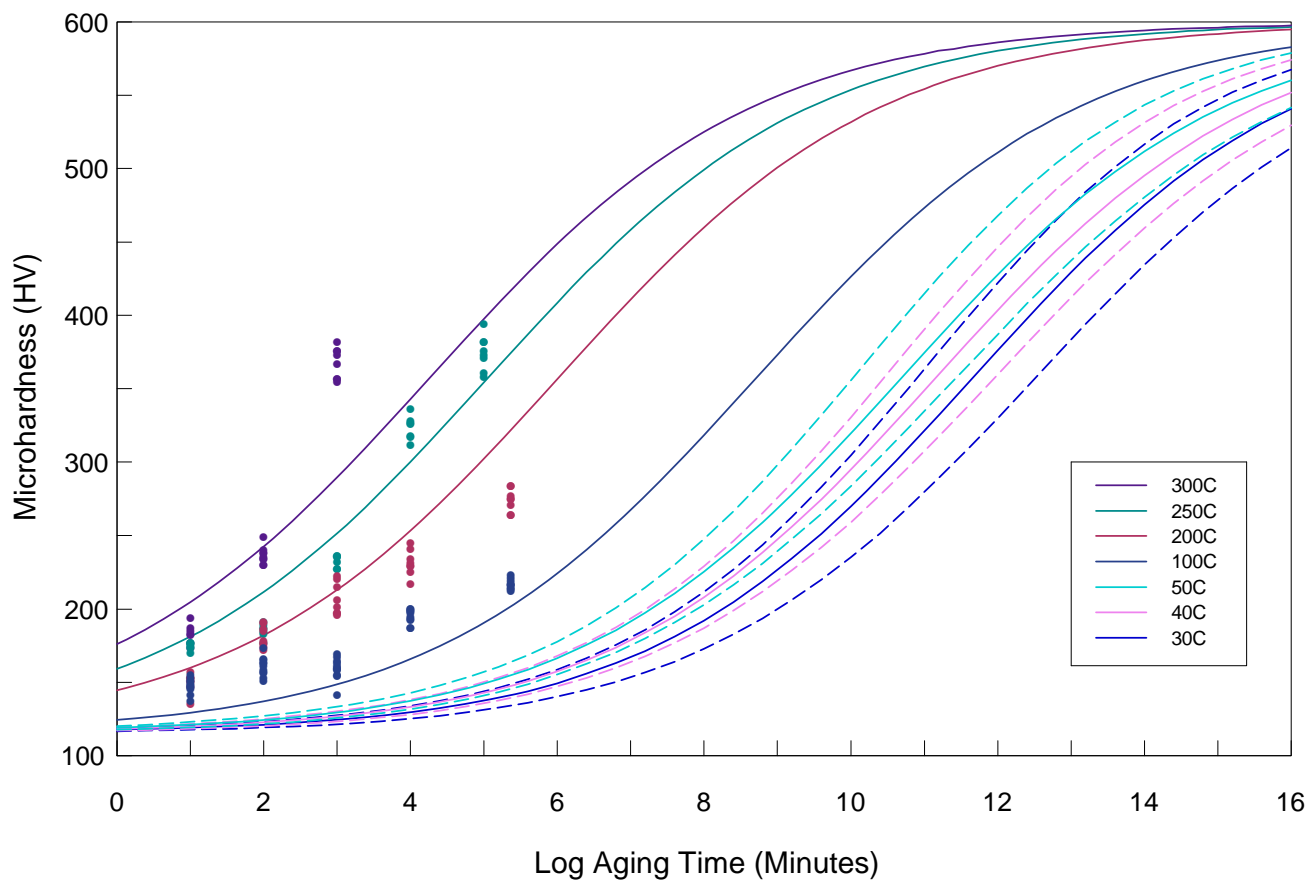

(b)

Figure A2.2. U-7.7Nb HV data (points), model fits to data (solid lines), and low-temperature model predictions (solid line-mean, dashed lines-95\% confidence intervals). (a) in terms of $f$, (b) in terms of absolute property. 
This page left blank intentionally. 


\section{APPENDIX 3: MODEL EVALUATION}

Residuals from a model fit are defined as the differences:

$$
e_{i}=y_{i}-\text { pred }_{i}
$$

where $y_{i}$ is an observed value for the ith replicate data point, and pred $_{i}$ is the corresponding mean fitted value from the model. In other words, the errors are the differences between what is observed and what is predicted based on the model. Thus, we can think of the $e_{i}$ as the observed errors if the model is correct. In regression analysis, it is assumed that the errors are independent, have a mean of 0 and a variance of $\sigma^{2}$, and follow a normal distribution. The assumption of the errors following a normal distribution is necessary for testing hypotheses about the model parameters and for computing confidence and prediction intervals. As part of the model evaluation process, residuals are examined to see if the assumptions hold. Plots of residuals from the models showed that the variance was constant over time, that successive error terms were independent, and that the errors appeared to follow a normal distribution. Normal probability plots of residuals plotted against the quartiles of a standard normal distribution are provided below in Figures A3.1 and A3.2. If the models provide an adequate fit to the data, then the points on these plots should fall along a straight line.

Another means of assessing the goodness of fit of a model is to look at the residual standard error $(R S E)$ of the fit. In equation form, this is defined as

$$
R S E=\sqrt{\sum_{i=1}^{n} \frac{\left(y_{i}-\text { pred }_{i}\right)^{2}}{(n-p)}}=\sqrt{\sum_{i=1}^{n} \frac{e_{i}^{2}}{(n-p)}},
$$

where the $e_{i}$ are the residuals from the model fit, and the quantity $(n-p)$ is the number of observations minus the number of parameters in the model, equal to the degrees of freedom ( $d f$ ). This value provides an overall summary of how well the model fits the observed data.

In the summary of the nonlinear model fit to the data, tests of hypotheses are examined to see whether or not the parameter estimates $(Q, A$, and $B)$ are significantly different from 0 . For the models chosen, all parameter estimates were significantly different from 0 , indicating that all three parameters were significant and relevant for consideration as part of the model fitting.

Various methods have been developed for investigating the goodness of fit of a nonlinear model. For a more complete discussion, see Ratkowsky [1983rat] or Bates and Watts [1988bat]. In particular, for nonlinear models, there are methods for measuring the degree to which the model and its individual parameter estimates exhibit linearity. The method used for assessing the degree of departure from linearity for the nonlinear model parameters in this report involved twodimensional profiles of the residual sum-of-squares function $\tau$. These plots are provided in Figures A3.3 and A3.4. For coordinate (as defined by each parameter) directions along which the approximate linear methods are accurate, a plot of the nonlinear t-statistic $\tau$ against the parameter estimate over several standard deviations on either side of the maximum likelihood estimate should produce a straight line. The degree to which such plots exhibit nonlinearity represent the degree of potential bias and inaccuracy in the parameter estimates. See Venables and Ripley [1994ven] for more details.

For most of the models, the two-dimensional profile for each of the parameters appears to be fairly linear. Where there is some degree of nonlinearity in one or more of the parameter 
estimates for a given property, the linear approximation for that parameter may be misleading. As a result, less confidence should be placed on these estimates.

Most notable among the exceptions to linearity in U-5.6Nb are the parameter estimates for $Q, A$, and $B$ for $1 Y M$.

Most notable among the exceptions to linearity in U-7.7Nb are the following parameter estimates:

1. $Q$ and $B$ for TE,

2. A for $1 Y M$,

3. A for UE, and

4. $Q$ and $B$ for $\mathrm{HV}$. 

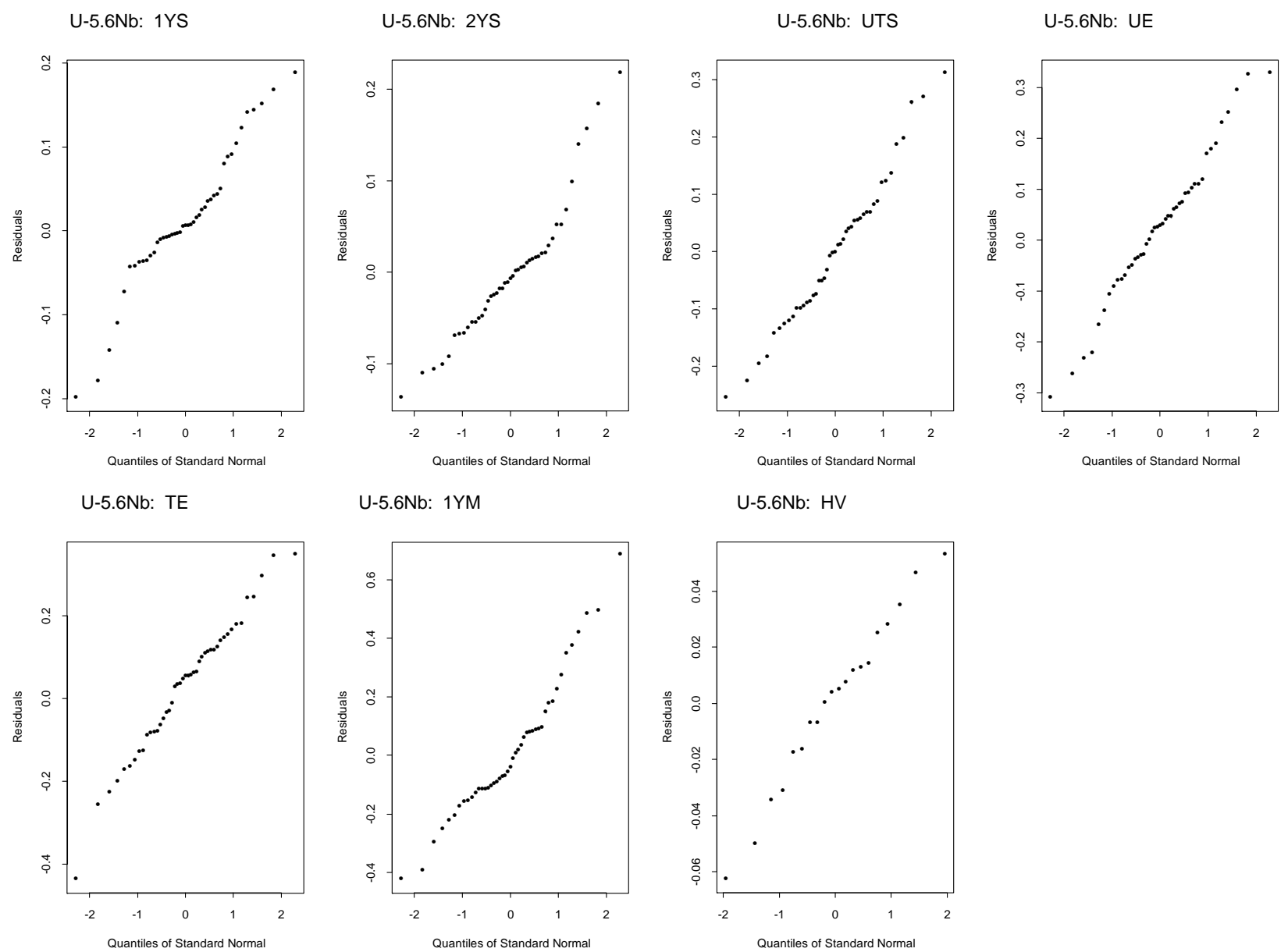

Figure A3.1. Normal probability plots of the residuals for U-5.6Nb model fits. 

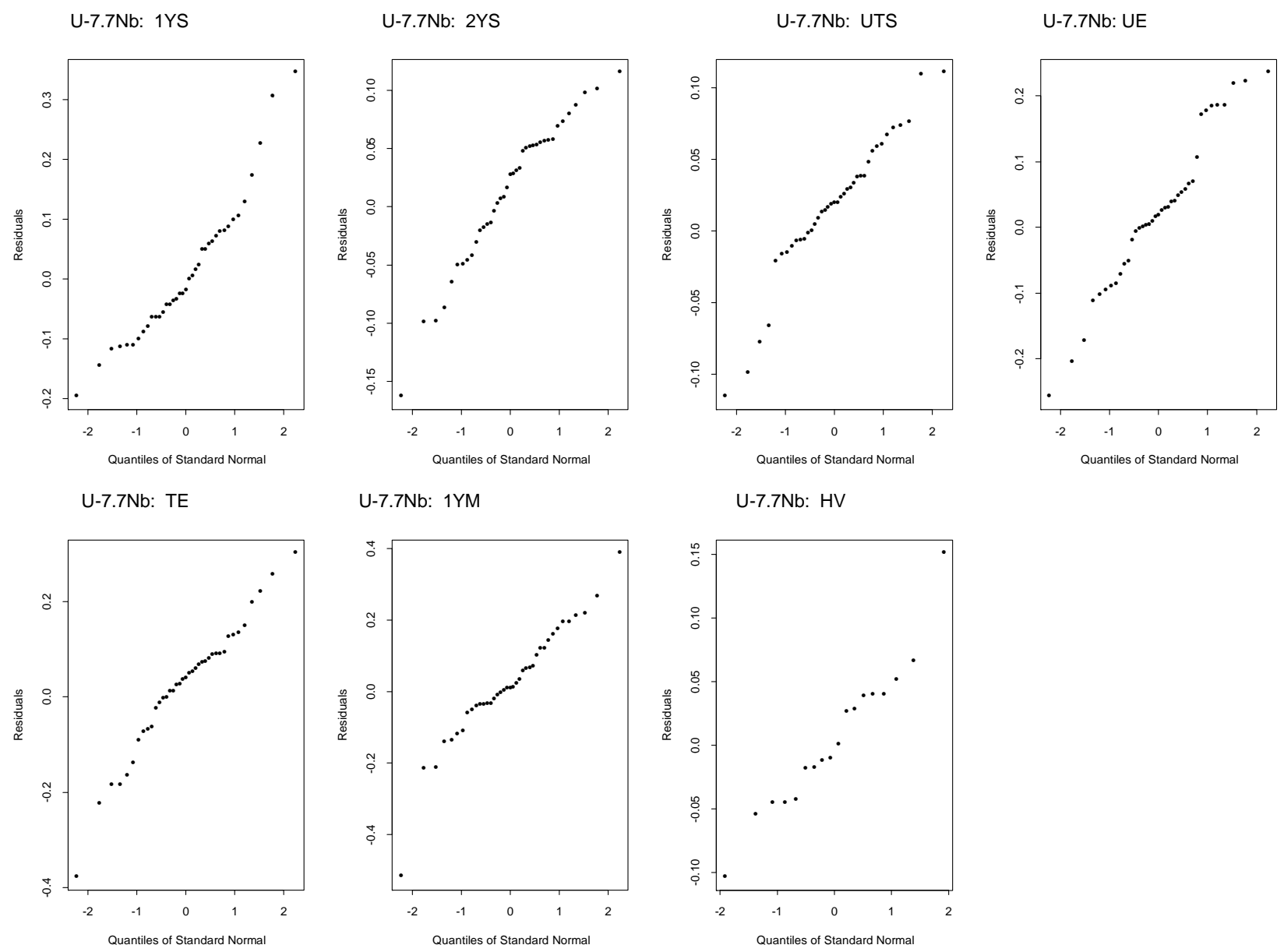

Figure A3.2. Normal probability plots of the residuals for U-7.7Nb model fits. 

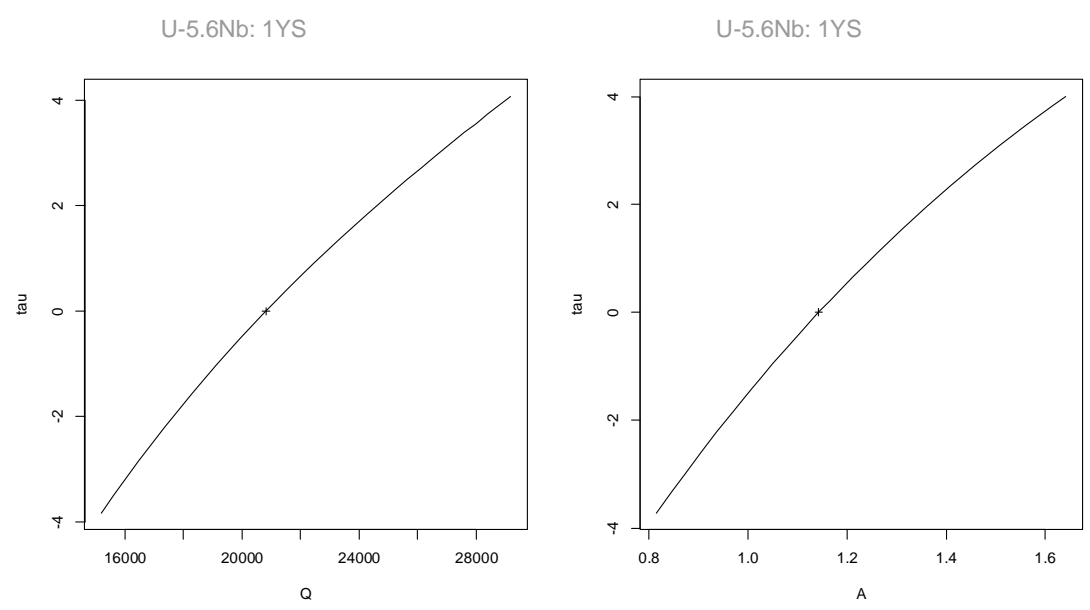

U-5.6Nb: 2YS

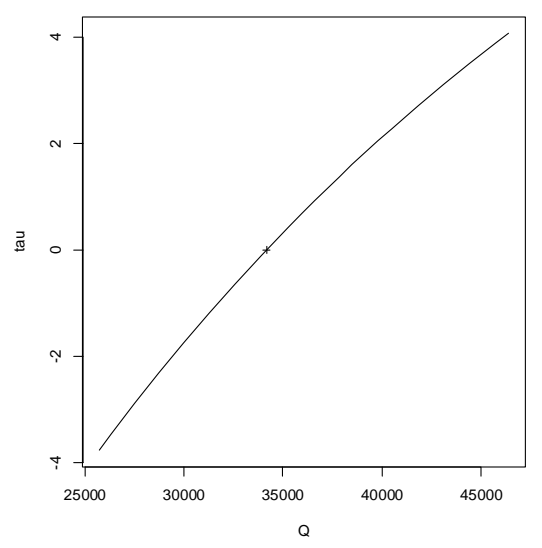

U-5.6Nb: $2 Y S$

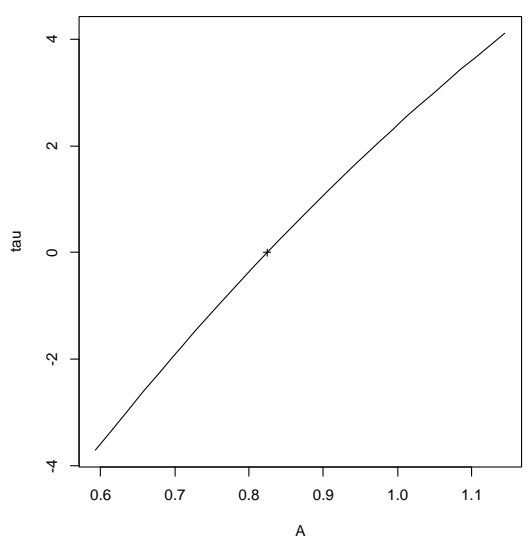

U-5.6Nb: 1YS

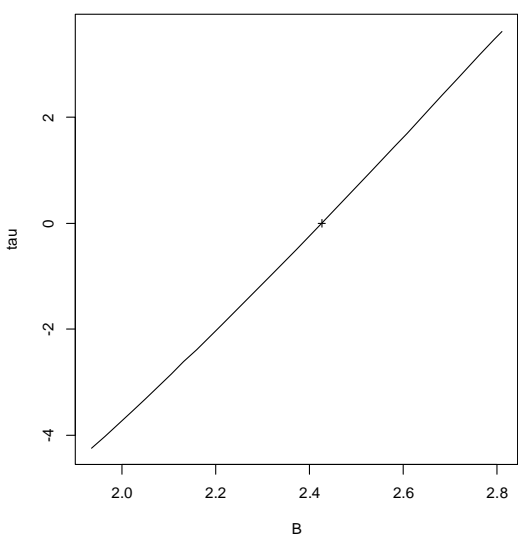

U-5.6Nb: $2 Y S$

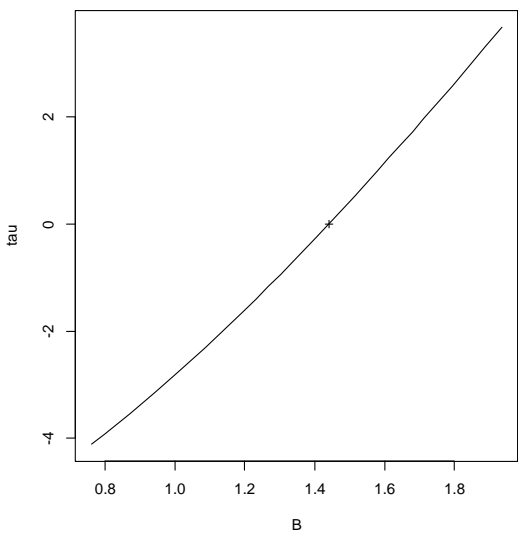

Figure A3.3. Two-dimensional profiles of the residual sum-of-squares function for U-5.6Nb model fits. 

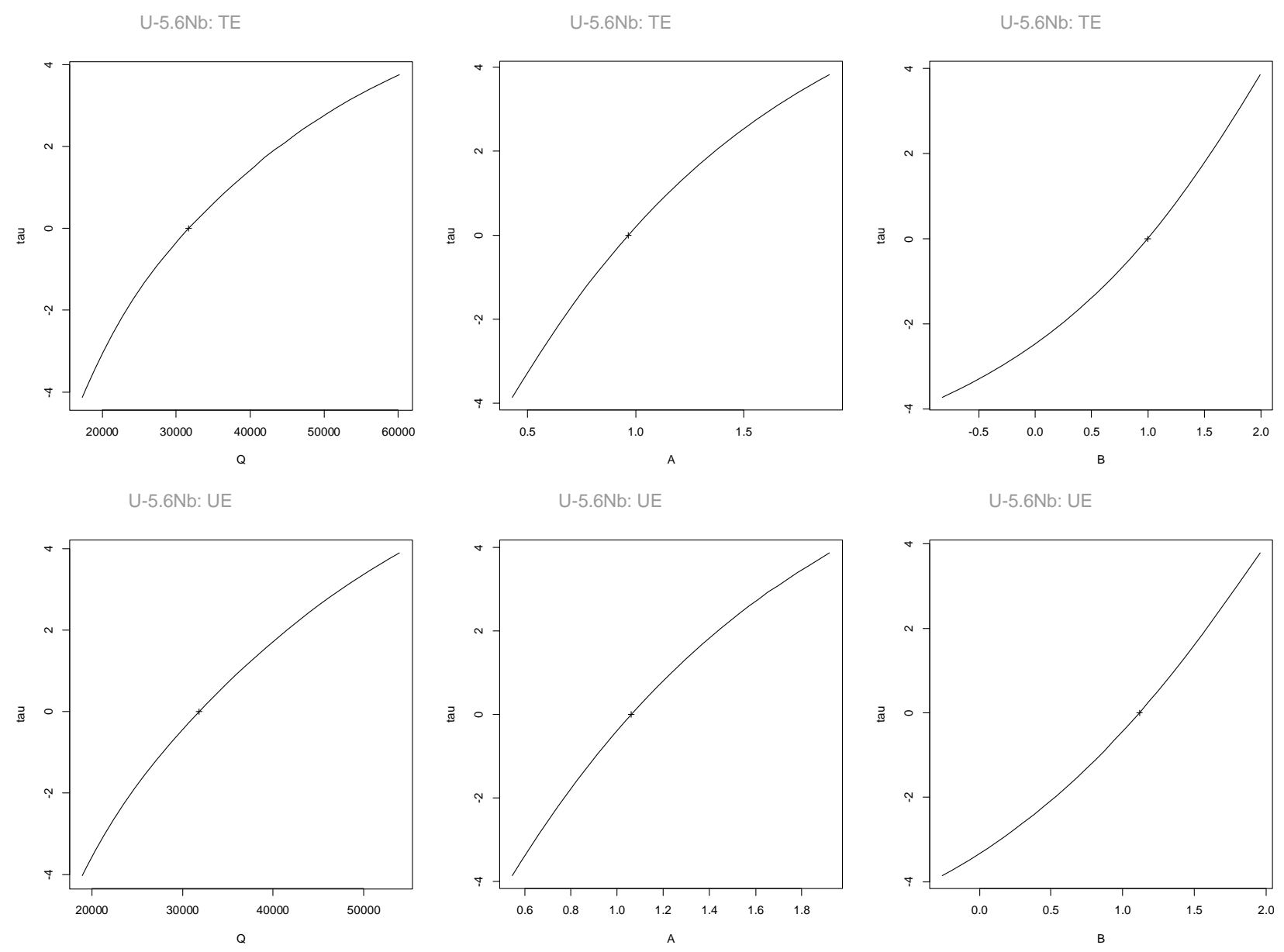

Figure A3.3 (continued). Two-dimensional profiles of the residual sum-of-squares function for U-5.6Nb model fits. 

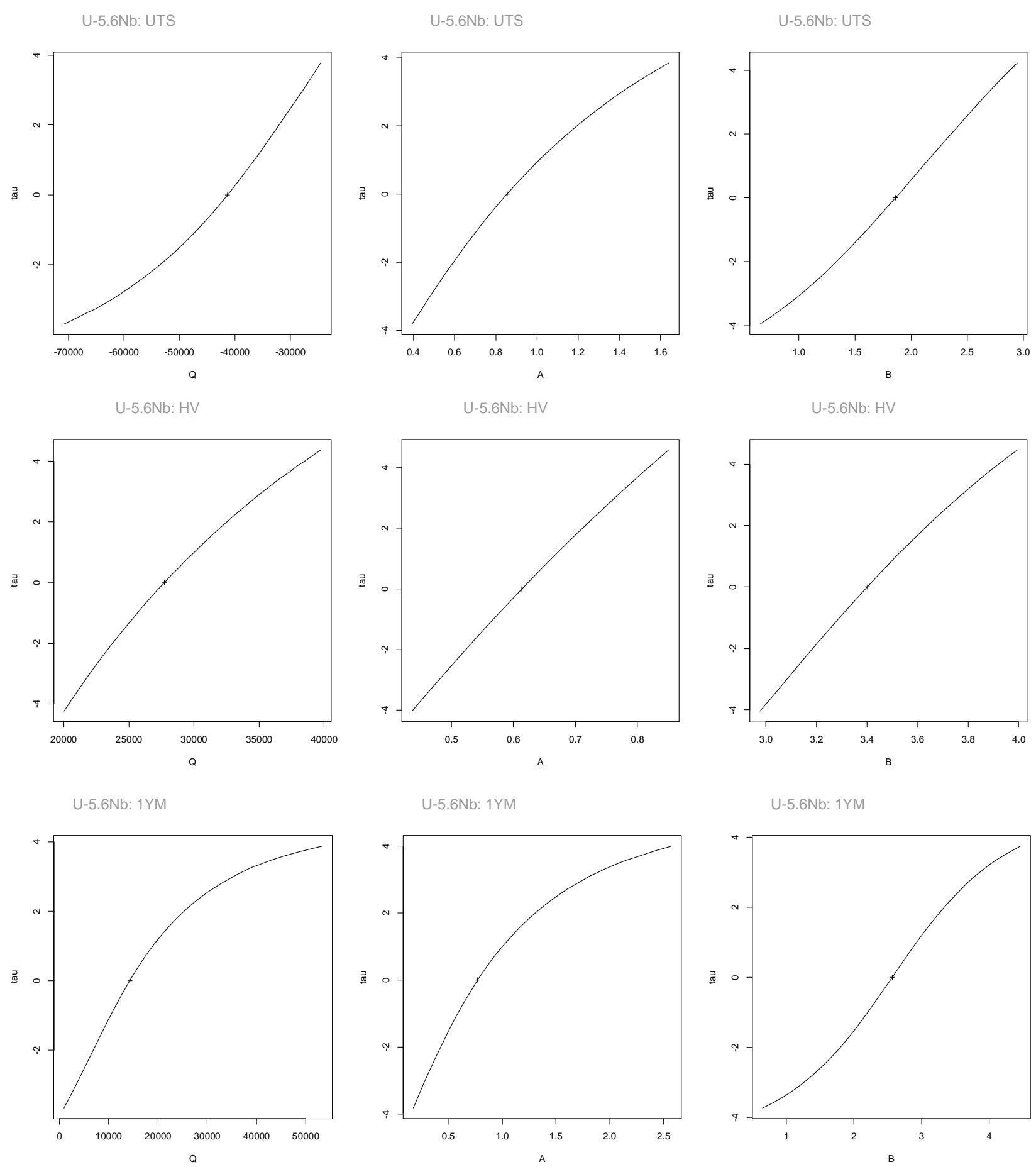

Figure A3.3 (continued). Two-dimensional profiles of the residual sum-of-squares function for U-5.6Nb model fits. 
U-7.7Nb: 1YS

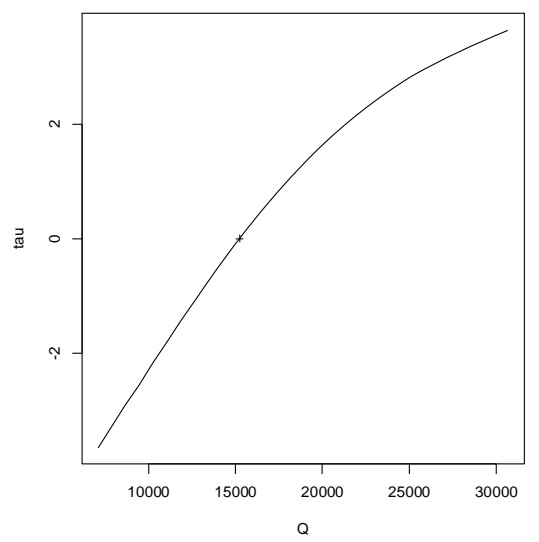

U-7.7Nb: 2YS

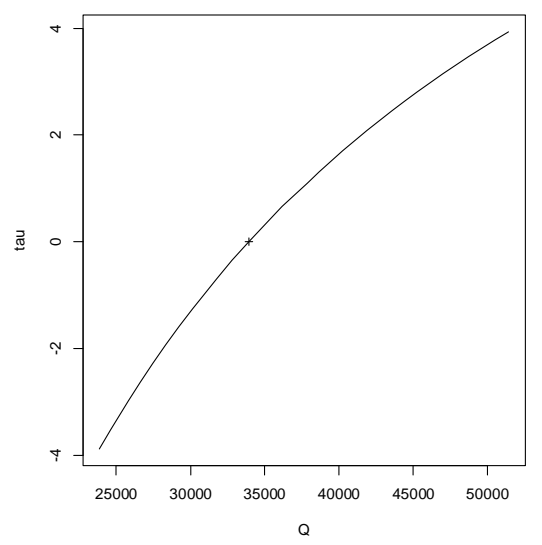

U-7.7Nb: 1YS

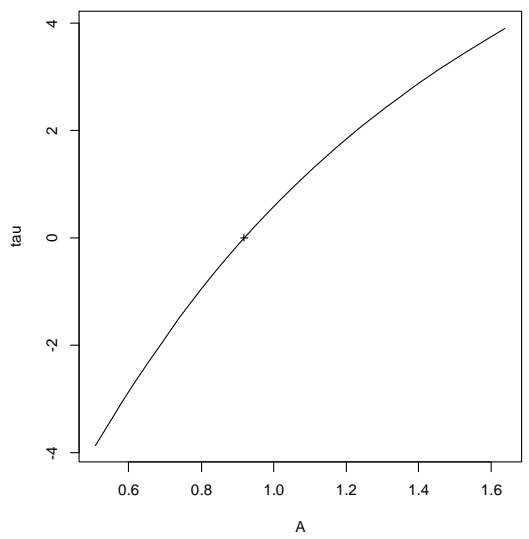

U-7.7Nb: 2YS

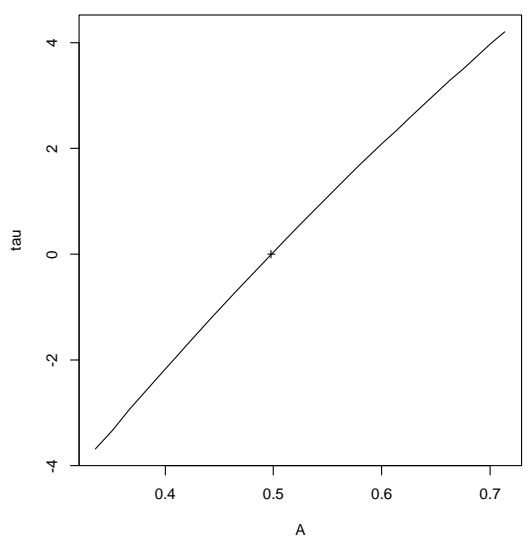

U-7.7Nb: 1YS

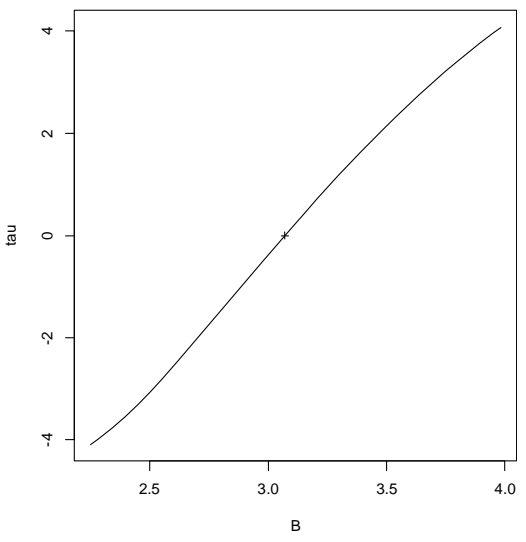

$\mathrm{U}-7.7 \mathrm{Nb}: 2 \mathrm{YS}$

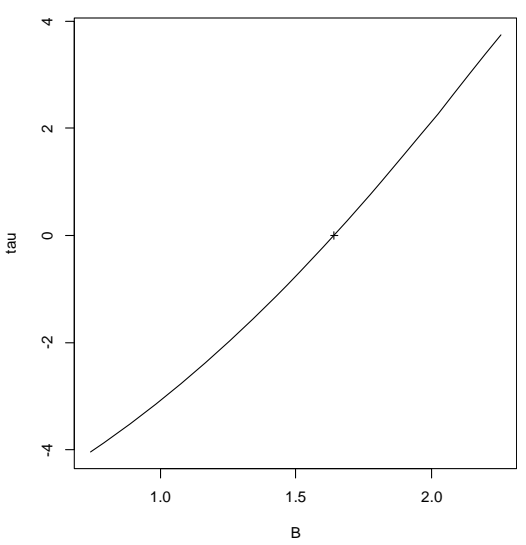

Figure A3.4. Two-dimensional profiles of the residual sum-of-squares function for U-7.7Nb model fits. 
U-7.7Nb: TE

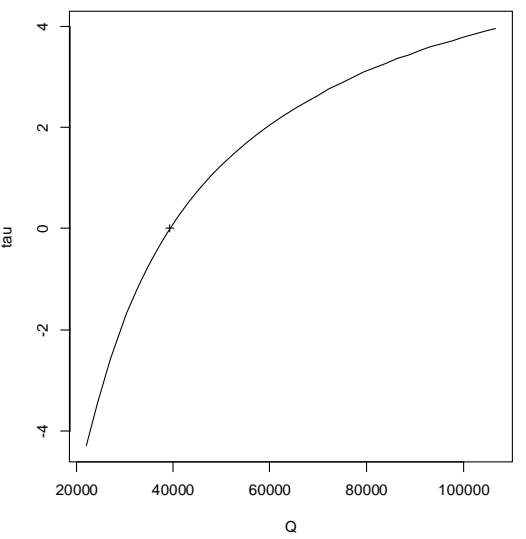

U-7.7Nb: UE

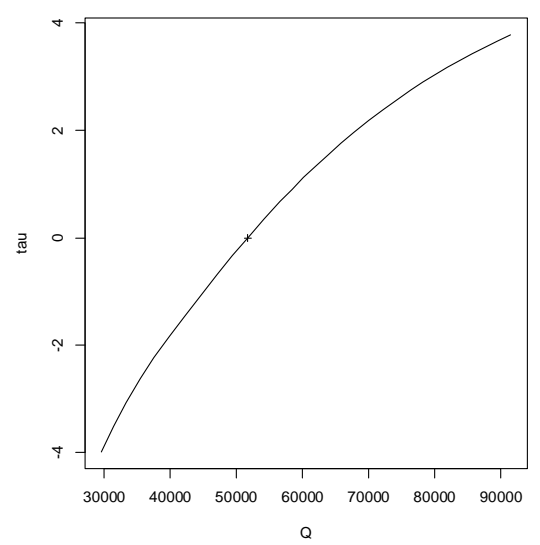

U-7.7Nb: TE

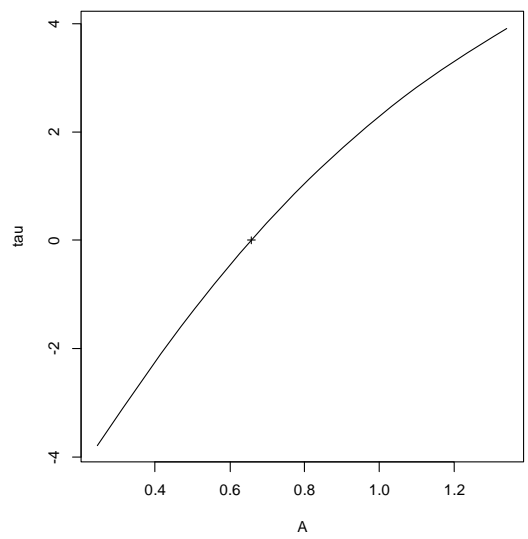

U-7.7Nb: UE

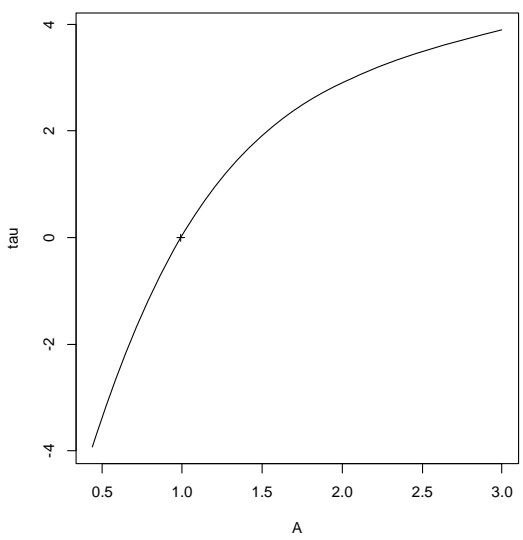

U-7.7Nb: TE

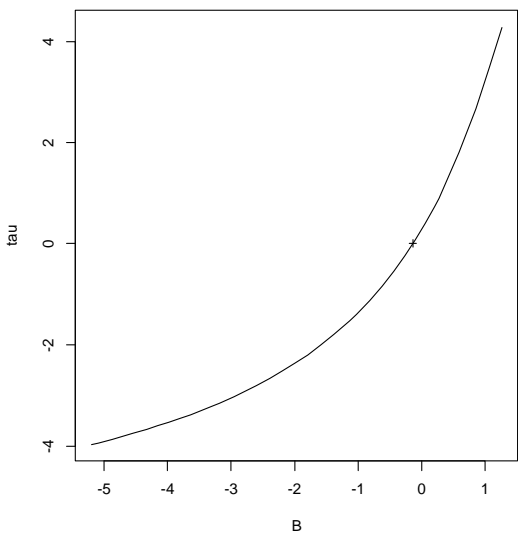

U-7.7Nb: UE

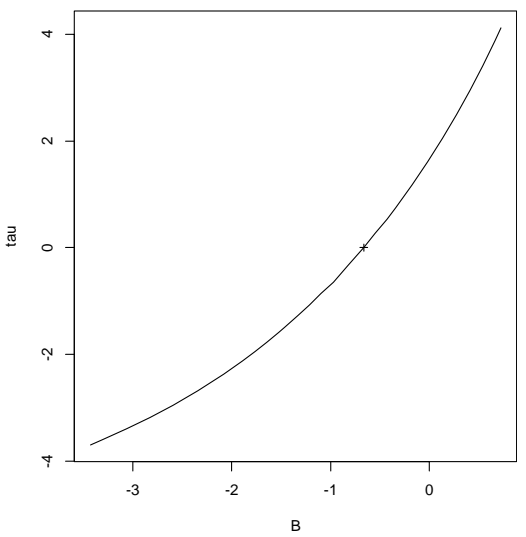

Figure A3.4 (continued). Two-dimensional profiles of the residual sum-of-squares function for U-7.7Nb model fits. 
U-7.7Nb: UTS

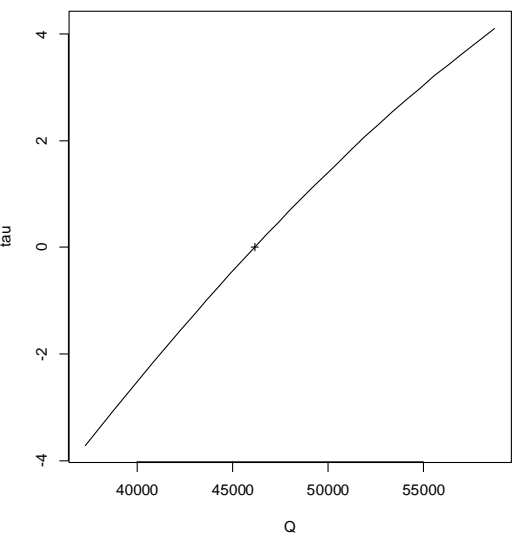

U-7.7Nb: HV

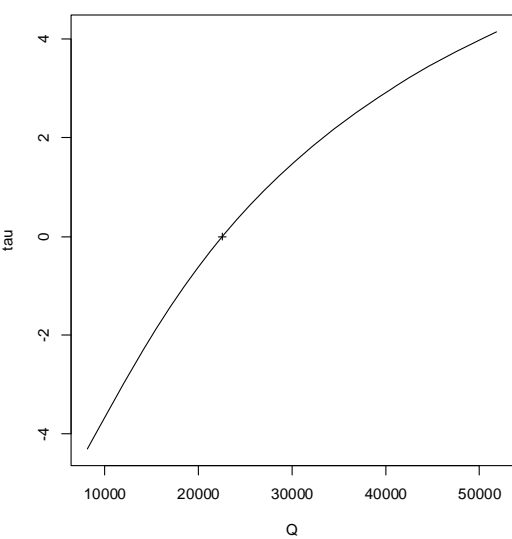

U-7.7Nb: 1YM

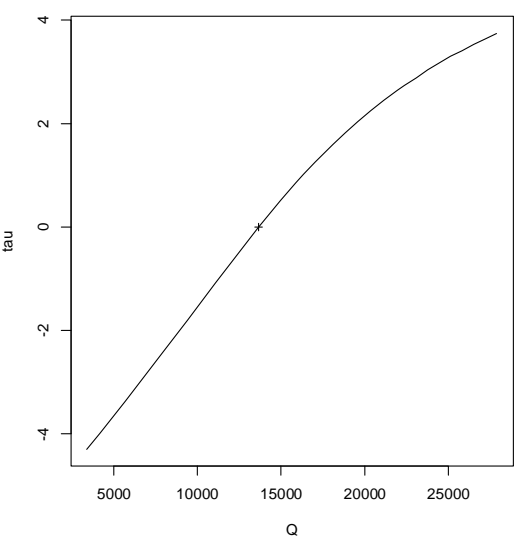

U-7.7Nb: UTS

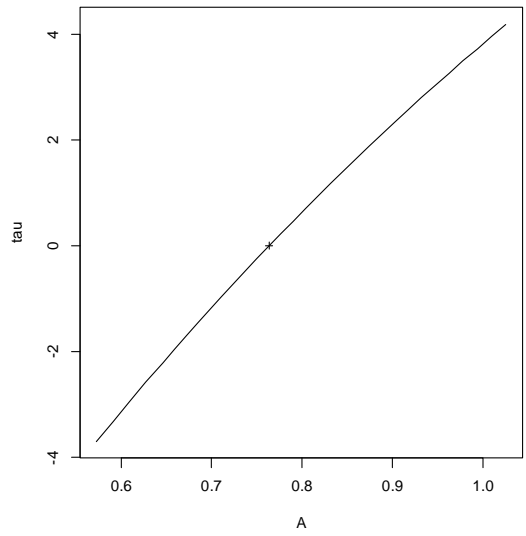

U-7.7Nb: HV

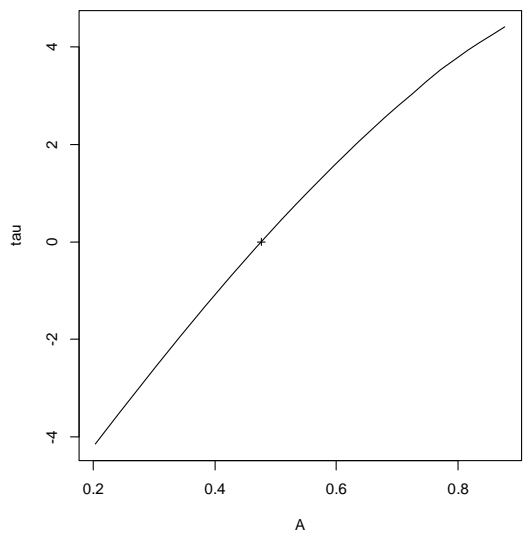

U-7.7Nb: 1YM

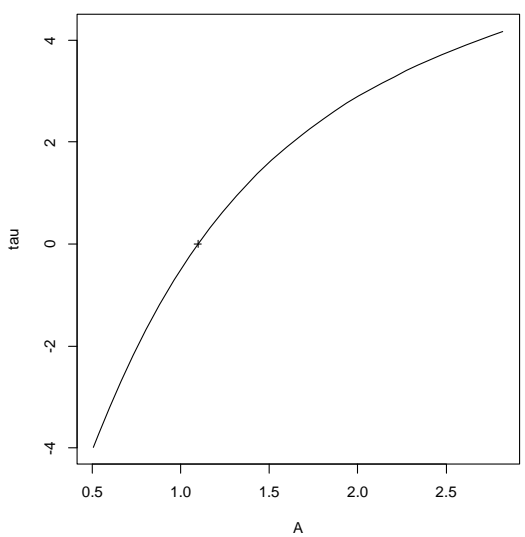

U-7.7Nb: UTS

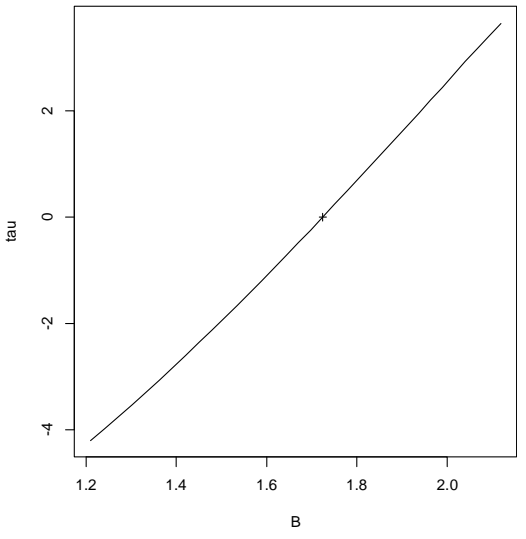

U-7.7Nb: HV

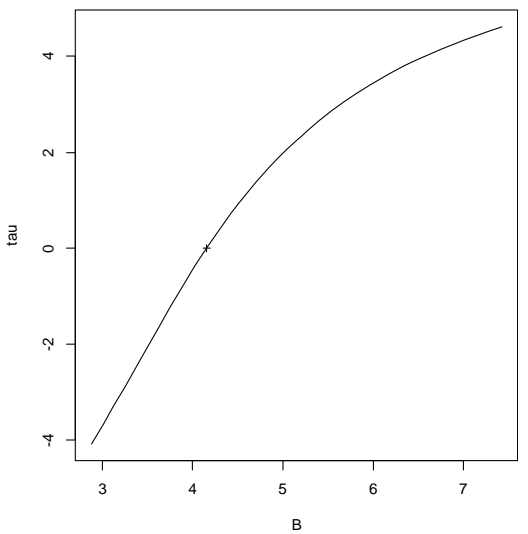

U-7.7Nb: $1 Y M$

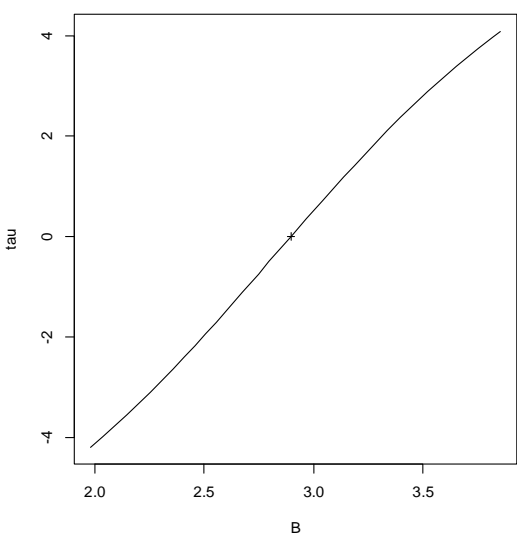

Figure A3.4 (continued). Two-dimensional profiles of the residual sum-of-squares function for U-7.7Nb model fits. 


\section{APPENDIX 4: BACKGROUND ON NONLINEAR MODELS}

\section{A4.1. Nonlinear Regression}

Consider the general form of a regression model:

$$
y_{i}=f\left(\boldsymbol{X}_{\boldsymbol{i}} ; \boldsymbol{\theta}\right)+\varepsilon_{i}
$$

where $\mathrm{y}_{i}$ represents the response variable, $\boldsymbol{X}_{i}$ represents a vector of explanatory variables, and $\varepsilon_{i}$ represents the error term at the $i$ th replicate data point, $i=(1,2, \ldots, n)$. The term $f\left(\boldsymbol{X}_{i}, \boldsymbol{\theta}\right)$ describes a function of the explanatory variables, represented by the vector $\boldsymbol{X}_{i}$, and the parameters, represented by the vector $\boldsymbol{\theta}$. The term $\varepsilon_{i} \sim \mathrm{N}\left(0, \sigma^{2}\right)$ means that the error is assumed to be normally distributed with mean zero and constant variance $\sigma^{2}$ for all values of the explanatory variables represented by $\boldsymbol{X}_{i}$. It is generally assumed that the errors are independent and identically distributed about the mean zero with constant variance. Independence means that the error at one value is not related to the error at some other value.

Given the validity of the assumption of independent and identically distributed normal error, one can make certain general statements about the least-squares estimators in linear and nonlinear regression models. Least-squares estimators of $\boldsymbol{\theta}$ are those that minimize the sums of squares of the deviations between the observed values and the predicted values from the model. The maximum likelihood estimates for the parameters in $\boldsymbol{\theta}$ are least squares estimates.

A nonlinear regression model is distinguished from a linear regression model in that at least one of its parameters appears nonlinearly. In the formal mathematical sense, nonlinear means that at least one of the derivatives of $\mathrm{y}_{i}$ with respect to the parameters in $\boldsymbol{\theta}$ is a function of at least one of those parameters.

In general, linear regression models are preferred to nonlinear regression models because of the desirable properties associated with their estimators. For linear models, the estimates of the parameters are unbiased, normally distributed, and have the minimum possible variance. The estimators for nonlinear models do not exhibit these properties, but achieve these properties only asymptotically as the sample size approaches infinity. In addition, exact standard errors and confidence intervals can be computed for linear models, whereas for nonlinear models, these values are only approximate.

For linear models, the estimators of the parameters can be obtained from explicit mathematical expressions, whereas for nonlinear regression models, either an iterative procedure using a mathematical algorithm or an exhaustive search procedure must be used for finding the optimum solution. One commonly used iterative procedure is the Gauss-Newton method.

For a linear model, the solution locus is a line or a plane through the origin and the coordinate system defined by the parameters. It has no curvature. On the other hand, the solution locus for a nonlinear model is a curved surface. The closer such a curved surface is to being linear, the closer the properties of the nonlinear model are to having the properties of a linear model. For such close-to-linear models, the predicted values of the response variable will be virtually unbiased. The least-squares estimators of the parameters will have distributions closely approximating a normal distribution. In addition, the confidence intervals for each parameter will be close to being exact. Additional information on nonlinear models can be found in Ratkowsky [1983rat], Bates and Watts [1988bat], and Draper and Smith [1981dra]. 


\section{A4.2. Application of Statistical Intervals}

The appropriate choice of a statistical interval depends on the general purpose of the interval and the characteristic of interest. One must decide whether the main interest is in describing the population from which the sample has been selected or in predicting the results of future samples from the same population. Intervals that describe the population include confidence intervals for the population mean or for mean fitted values (such as in regression modeling). In contrast, prediction intervals deal with predicting (or containing) the results of a future sample from the population. See Hahn and Meeker [1982mee, 1991hah] for a more thorough discussion on different types of intervals.

A confidence interval is an interval that can be claimed to contain the mean of a population with a high degree of confidence. For a 95\% confidence interval on the mean fitted values of a regression model, it can be said that, for very many repeated experiments, in the long run, one would be correct approximately $95 \%$ of the time in claiming that the true regression fit to the population is contained in the calculated confidence interval.

A prediction interval for a single future observation is an interval that will, with a specified degree of confidence, contain the next randomly selected observation from the population. This interval is calculated from the sample data under the important assumption that the previously sampled items and the future ones can be regarded as random samples from the same population. It also assumes similar aging conditions and measurement procedures. If, for example, three future measurements are to be obtained at a specified point in time, then, in the long run after many repeated experiments, one would be correct $95 \%$ of the time in claiming that the true values of the future samples, as well as the average of the three samples, would be contained within a 95\% prediction interval if the model is truly representative of the population being studied. If a validation point were to fall outside of the prediction interval bounds, then one could conclude either that the sample is different from the population from which the model was obtained, or that the model does not adequately represent the population.

Tables 4.7 to 4.13 contain mean fitted values from the nonlinear regression models and corresponding confidence intervals on these predictions for the times and temperatures both within and outside of the time-temperature space spanned by the model-fitting data. In addition, these tables contain measured values which are the results of ongoing and future validation experiments, occurring at temperatures observed in the first experiment and at temperatures lower than previously studied. These values will be referred to as validation points. For the validation points, prediction intervals are provided. In Tables 4.7 to 4.13 , both the confidence intervals on the mean fitted values from the model and the prediction intervals for the validation points have been truncated at the lower and upper bounds for each property (Table 3.1). These intervals have also been truncated in the plots showing the location of the validation points relative to the predicted curves and bounds.

\section{A4.3. Computation of Statistical Intervals}

Assuming that the errors from the model fit are normally distributed, confidence intervals on population parameters can be calculated. The first step in the process of obtaining an approximate confidence interval for the predicted nonlinear model is to obtain the standard errors of the fitted points. To compute these standard errors, the variance-covariance matrix and the equations for the first partial derivatives (gradients) of the model with respect to the three parameters are needed. The gradient equations are provided below, and the variance-covariance 
matrix values are provided in Table A4.1. At a given aging time and temperature, the equation for computing the standard error of a fitted value, se.fit, is as follows:

$$
\text { se. fit }=\sqrt{P^{T} * V C V * P}
$$

where $P$ is a $3 \times 1$ vector containing the first partial derivatives for the estimates of $Q, A$, and $B$, evaluated at a particular time and temperature, $P^{T}$ is the transpose of the vector $P$, and $V C V$ is a $3 \times 3$ matrix containing the variances for the parameters $Q, A$, and $B$ along its diagonal and the covariances between parameters on the off-diagonal.

First derivative equations were obtained using S-Plus software licensed by Insightful Corp. See Venables and Ripley [1994ven] for details on the software code. In order to keep the gradient equations readable, the equations have been broken down into the following six expressions:

$$
\begin{gathered}
U=\frac{\log (e)}{R} \approx \frac{0.43429}{1.99 \mathrm{cal} / \mathrm{mol}}=0.218 \mathrm{~mol} / \mathrm{cal} \\
V=\frac{1}{573.15 \mathrm{~K}}-\frac{1}{T} \\
W=x+Q U V-B \\
X=e^{A W} \\
Y=1+X \\
Z=Y^{2}
\end{gathered}
$$

where $A, B$, and $Q$ are the Logistic model parameters, $T$ is in Kelvin, and $x$ is $\log _{10}$ (time) as defined in Eq. 3.3.

The gradient equations are as follows:

$$
\begin{gathered}
\operatorname{grad}(Q)=\frac{A U V X}{Z} \\
\operatorname{grad}(A)=\frac{W X}{Z} \\
\operatorname{grad}(B)=-\frac{A X}{Z}
\end{gathered}
$$

The general procedure for computing an approximate lower and upper confidence interval at a given point involves the following steps:

1. Choose a value for time and a value for temperature.

2. Evaluate the gradient equations at the chosen time and temperature. (This produces a $P$ vector.)

3. Compute the standard error using Eq. A4.2.

4. Use the following equation to compute an approximate confidence interval:

$$
C I=\text { pred }_{i} \pm t_{(0.95, d f)} * \text { se. fit }{ }_{i} .
$$


In this equation,

- $\quad \operatorname{pred}_{i}$ is the predicted or mean fitted value from the model at time $i$ for the chosen temperature,

- $t_{(0.95, d f)}$ is the t-statistic for a 95\% confidence level with $d f$ degrees of freedom,

- $d f$ (degrees of freedom) is computed as the total number of replicate observations in the data set $(n)$ minus the number of parameter estimates $(p)$, and

- $\quad s_{\text {. }} \mathrm{fit}_{i}$ is the computed standard error of the fitted value at time $i, \mathrm{Eq}$. A4.2.

The variance-covariance matrices are presented in lower triangular form in Table A4.1.

A prediction interval is wider than a confidence interval. One would expect that an estimate of an individual measurement would have more variability than an estimate of the mean response from a group of measurements. In computing a prediction interval on an actual observed $y_{i}$, there is an additional source of error. It represents the deviation of the individual $y_{i}$ values from the mean fitted values from the model. See Kleinbaum and Kupper [1978kle] for more details on the calculation of a prediction interval for regression.

In equation form, the calculation of a prediction interval is as follows:

$$
P I=\operatorname{pred}_{i} \pm t_{(0.95, d f)} \sqrt{\left(\text { se. } f t_{i}\right)^{2}+\left(\frac{R S E}{n v}\right)^{2}},
$$

where $\operatorname{pred}_{i}, t_{(0.95, d f)}$, and se.fit ${ }_{\mathrm{i}}$ are as defined above. The equation for computing the residual standard error is as follows:

$$
R S E=\sqrt{\sum_{i=1}^{n} \frac{\left(y_{i}-\text { pred }_{i}\right)^{2}}{(n-p)}},
$$

where $n$ is the number of samples, $p$ is the number of parameters in the model, and $n v$ is the number of future validation points for a given alloy and temperature combination. The RSE values for each model are provided in Table 4.3. 
Table A4.1 Variance-Covariance Matrices

U-5.6Nb TE

\begin{tabular}{|c|c|c|c|}
\hline & $Q$ & $A$ & $B$ \\
\hline$Q$ & 24852120 & & \\
\hline$A$ & -311.5718 & 0.03008261 & \\
\hline$B$ & -1259.116 & 0.01844140 & 0.09262537 \\
\hline
\end{tabular}

U-7.7Nb TE

\begin{tabular}{|c|c|c|c|}
\hline & $Q$ & $A$ & $B$ \\
\hline$Q$ & 39500890 & & \\
\hline$A$ & -502.1799 & 0.01500349 & \\
\hline$B$ & -2690.861 & 0.04079818 & 0.2308611 \\
\hline
\end{tabular}

U-5.6Nb UE

\begin{tabular}{|c|c|c|c|}
\hline & $Q$ & $A$ & $B$ \\
\hline$Q$ & 18541480 & & \\
\hline$A$ & -199.8689 & 0.02822474 & \\
\hline$B$ & -891.2887 & 0.01132047 & 0.06320916 \\
\hline
\end{tabular}

U-7.7Nb UE

\begin{tabular}{|c|c|c|c|}
\hline & $Q$ & $A$ & $B$ \\
\hline$Q$ & 41153030 & & \\
\hline$A$ & -471.5211 & 0.02680211 & \\
\hline$B$ & -2561.475 & 0.03651868 & 0.1844366 \\
\hline
\end{tabular}

U-5.6Nb 1YS

\begin{tabular}{|c|c|c|c|}
\hline & $Q$ & $A$ & $B$ \\
\hline$Q$ & 3224415 & & \\
\hline$A$ & -37.06893 & 0.009140565 & \\
\hline$B$ & -150.8623 & 0.0006613776 & 0.01281954 \\
\hline
\end{tabular}

U-7.7Nb 1YS

\begin{tabular}{|c|c|c|c|}
\hline & $Q$ & $A$ & $B$ \\
\hline$Q$ & 4855980 & & \\
\hline$A$ & -64.74975 & 0.015668949 & \\
\hline$B$ & -269.6162 & -0.002011677 & 0.03180668 \\
\hline
\end{tabular}

U-5.6Nb 1YM

\begin{tabular}{|c|c|c|c|}
\hline & $Q$ & $A$ & $B$ \\
\hline$Q$ & 16677440 & & \\
\hline$A$ & -248.2645 & 0.03324399 & \\
\hline$B$ & -1041.423 & 0.001828075 & 0.1348329 \\
\hline
\end{tabular}


Table A4.1 (continued).

U-7.7Nb 1YM

\begin{tabular}{|c|c|c|c|}
\hline & $Q$ & $A$ & $B$ \\
\hline$Q$ & 5346451 & & \\
\hline$A$ & -73.02778 & 0.03572418 & \\
\hline$B$ & -338.8812 & -0.0005889329 & 0.04206774 \\
\hline
\end{tabular}

U-5.6Nb 2YS

\begin{tabular}{|c|c|c|c|}
\hline & $Q$ & $A$ & $B$ \\
\hline$Q$ & 6605678 & & \\
\hline$A$ & -58.80722 & 0.004762360 & \\
\hline$B$ & -301.4772 & 0.002406113 & 0.02084868 \\
\hline
\end{tabular}

U-7.7Nb 2YS

\begin{tabular}{|c|c|c|c|}
\hline & $Q$ & $A$ & $B$ \\
\hline$Q$ & 9230194 & & \\
\hline$A$ & -83.27905 & 0.002097209 & \\
\hline$B$ & -416.9067 & 0.003069187 & 0.03066215 \\
\hline
\end{tabular}

U-5.6Nb UTS

\begin{tabular}{|c|c|c|c|}
\hline & $Q$ & $A$ & $B$ \\
\hline$Q$ & 24835420 & & \\
\hline$A$ & 285.3534 & 0.02084541 & \\
\hline$B$ & 905.9384 & 0.003800227 & 0.05845111 \\
\hline
\end{tabular}

U-7.7Nb UTS

\begin{tabular}{|c|c|c|c|}
\hline & $Q$ & $A$ & $B$ \\
\hline$Q$ & 6724107 & & \\
\hline$A$ & -64.77024 & 0.002894096 & \\
\hline$B$ & -228.1218 & 0.001549427 & 0.01245295 \\
\hline
\end{tabular}

U-5.6Nb HV

\begin{tabular}{|c|c|c|c|}
\hline & $Q$ & $A$ & $B$ \\
\hline$Q$ & 5122572 & & \\
\hline$A$ & -53.36997 & 0.002207797 & \\
\hline$B$ & -91.16910 & -0.002040067 & 0.013020668 \\
\hline
\end{tabular}

U-7.7Nb HV

\begin{tabular}{|c|c|c|c|}
\hline & $Q$ & $A$ & $B$ \\
\hline$Q$ & 16340840 & & \\
\hline$A$ & -114.2892 & 0.005124729 & \\
\hline$B$ & -416.0291 & -0.01342360 & 0.1063440 \\
\hline
\end{tabular}


This page left blank intentionally. 
This page left blank intentionally. 
This report has been reproduced directly from the best available copy. It is available electronically on the Web (http://www.doe.gov/bridge).

Copies are available for sale to U.S. Department of Energy employees and contractors from:

Office of Scientific and Technical Information

P.O. Box 62

Oak Ridge, TN 37831

(865) 576-8401

Copies are available for sale to the public from: National Technical Information Service

U.S. Department of Commerce

5285 Port Royal Road

Springfield, VA 22161

(800) 553-6847 
- Los Alamos NATIONAL LABORATORY

EST.1943 